\title{
Selective stimulation of the subthalamic nucleus in Parkinson's disease: dream or near future
}

Citation for published version (APA):

Janssen, M. L. F. (2015). Selective stimulation of the subthalamic nucleus in Parkinson's disease: dream or near future. [Doctoral Thesis, Maastricht University]. Datawyse / Universitaire Pers Maastricht. https://doi.org/10.26481/dis.20150513mj

Document status and date:

Published: 01/01/2015

DOI:

10.26481/dis.20150513mj

Document Version:

Publisher's PDF, also known as Version of record

\section{Please check the document version of this publication:}

- A submitted manuscript is the version of the article upon submission and before peer-review. There can be important differences between the submitted version and the official published version of record.

People interested in the research are advised to contact the author for the final version of the publication, or visit the DOI to the publisher's website.

- The final author version and the galley proof are versions of the publication after peer review.

- The final published version features the final layout of the paper including the volume, issue and page numbers.

Link to publication

\footnotetext{
General rights rights.

- You may freely distribute the URL identifying the publication in the public portal. please follow below link for the End User Agreement:

www.umlib.nl/taverne-license

Take down policy

If you believe that this document breaches copyright please contact us at:

repository@maastrichtuniversity.nl

providing details and we will investigate your claim.
}

Copyright and moral rights for the publications made accessible in the public portal are retained by the authors and/or other copyright owners and it is a condition of accessing publications that users recognise and abide by the legal requirements associated with these

- Users may download and print one copy of any publication from the public portal for the purpose of private study or research.

- You may not further distribute the material or use it for any profit-making activity or commercial gain

If the publication is distributed under the terms of Article $25 \mathrm{fa}$ of the Dutch Copyright Act, indicated by the "Taverne" license above, 


\section{Selective stimulation of the subthalamic nucleus in Parkinson's disease}

\section{dream or near future?}

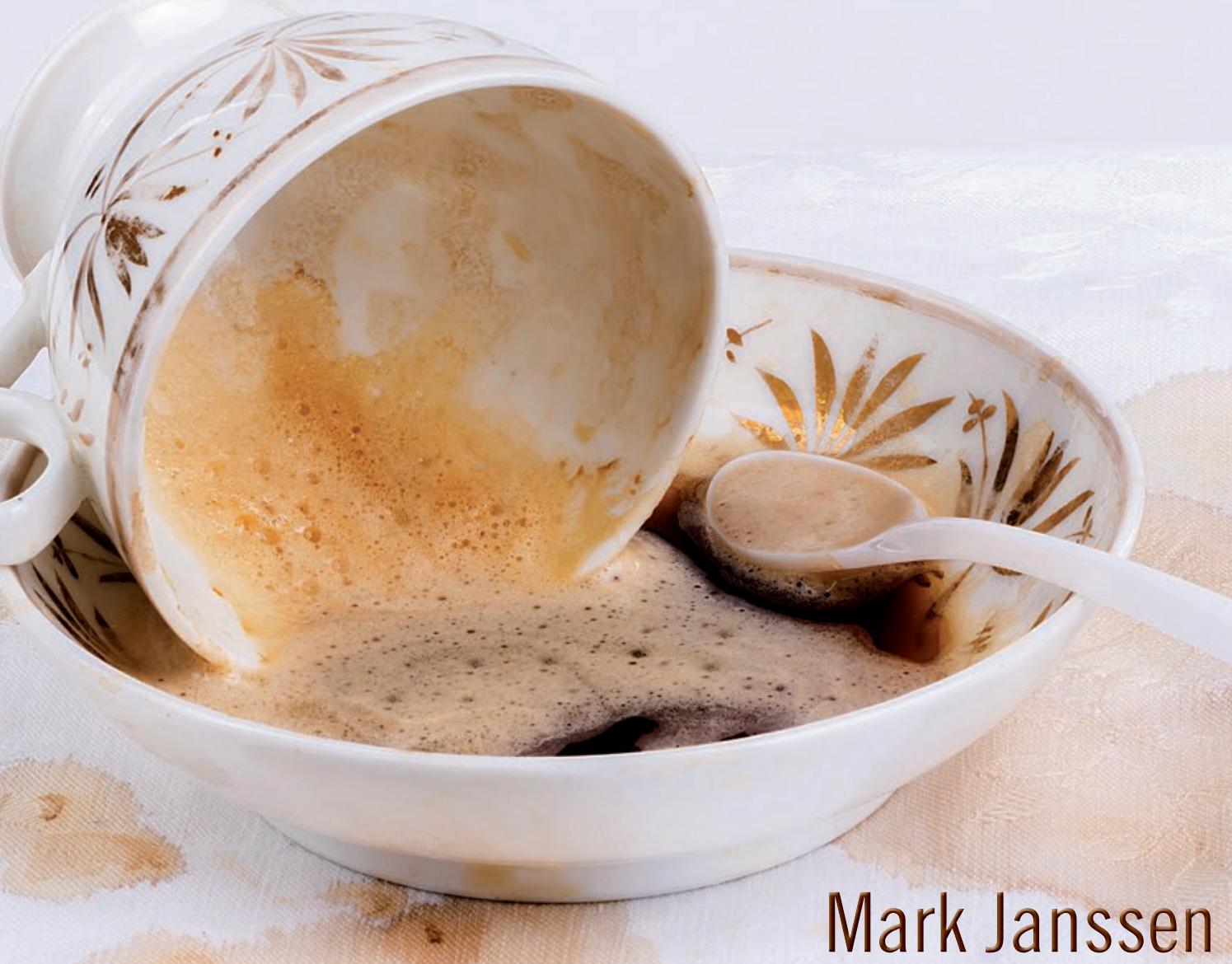


Aan mijn ouders

Foto omslag: Yara Verstappen

Productie: Datawyse | Universitaire Pers Maastricht

ISBN 9789461594358

(C) Copyright MLF Janssen, Maastricht 2015 


\title{
Selective stimulation of the subthalamic nucleus in Parkinson's disease
}

\author{
dream or near future?
}

\author{
ter verkrijging van de graad van doctor aan de Universiteit Maastricht, \\ op gezag van de Rector Magnificus, Prof. dr L.L.G. Soete \\ volgens het besluit van het College van Decanen, \\ in het openbaar te verdedigen \\ op woensdag 13 mei 2015 om 16.00 uur
}

door

\section{Marcus Leo Franciscus Janssen}

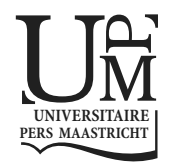




\section{Promotores}

Prof. dr. Y. Temel

Prof. dr. V. Visser-Vandewalle

Prof. dr. A. Benazzouz, Institut des Maladies Neurodégénératives, Bordeaux, France.

\section{Beoordelingscommissie}

Prof. dr. R. Van Oostenbrugge (voorzitter)

Dr. R. Esselink, UMC St Radboud Nijmegen

Prof. dr. B. Falkenburger, RWTH University Aachen, Germany

Prof. dr. W. Mess

Prof. dr J.J. Van Overbeeke 


\section{Content}

Abbreviations 7

General introduction

Parkinson's disease, the subthalamic nucleus and deep brain stimulation.

CHAPTER 1 Subthalamic nucleus high frequency stimulation for advanced Parkinson's disease: motor and neuropsychological outcome after 10 years.

CHAPTER 2 High frequency stimulation of the subthalamic nucleus increases c-Fos immunoreactivity in the dorsal raphe nucleus and afferent brain regions

CHAPTER 3 Cortico-subthalamic projections in the rat.

CHAPTER 4 The antidepressant effects of ventromedial prefrontal cortex is associated with neural activation in the medial part of the subthalamic nucleus

CHAPTER 5 Functional cortico-subthalamic inputs from the motor, limbic and associative areas in normal and dopamine depleted rats

CHAPTER 6 Subthalamic Neuronal Responses to Cortical Stimulation 105

CHAPTER 7 Cortically evoked potentials in the human subthalamic nucleus

CHAPTER 8 Automated gait analysis in bilateral Parkinsonian rats and the role of L-DOPA therapy

CHAPTER 9 Mild dopaminergic lesions are accompanied by robust changes in subthalamic nucleus activity

DISCUSSION 165 


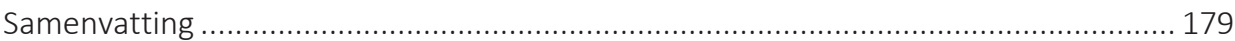

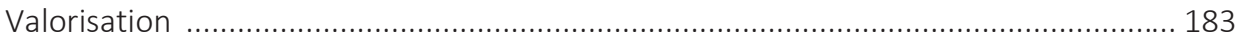

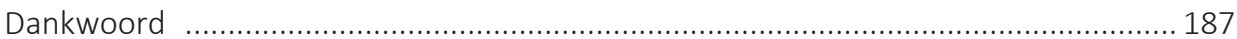

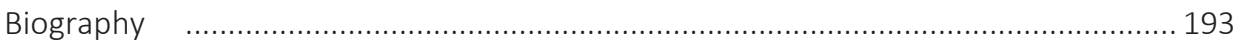

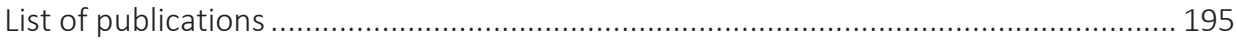

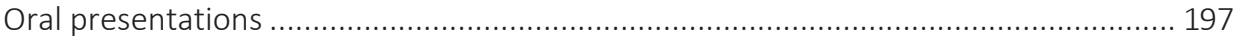

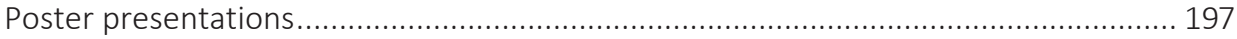




\section{Abbreviations}

Al agranular insular

ADL activities of daily living

AP anteroposterior

BDA biotinylated dextran amine

BDI Beck depression inventory

c-Fos-ir c-Fos immunoreactivity

$\mathrm{Cg} \quad$ cingulate gyrus

CMA cingulate motor area

COX cytochrome C oxidase

$\mathrm{CPu} \quad$ caudate putamen

CVLT California verbal learning test

DA dopamine

DAB 3,3-diaminobenzidine

DBS deep brain stimulation

DRN dorsal raphe nucleus

DTI diffusing tensor imaging

EP entepeduncular nucleus

FST forced swim test

GABA $\quad y$-aminobutyric acid

GP globus pallidus

GPe globus pallidus externus

GPi globus pallidus internus

HFS high frequency stimulation

HRP horseradish peroxide

IFC inferior frontal cortex

IL infralimbic

LED levodopa equivalent dose

LFP local field potential

$\mathrm{LHb} \quad$ lateral habenula

LSD least significant difference

MC motor cortex

MCS motor cortex stimulation

$\mathrm{ML} \quad$ mediolateral

MMSE mini mental state examination

MPFC medial prefrontal cortex

MSA multi system atrophy 


$\begin{array}{ll}\text { MT } & \text { motor time } \\ \text { M1 } & \text { lateral agranular cortex; primary motor cortex } \\ \text { NAC } & \text { nucleus accumbens } \\ \text { NiCL }_{2} & \text { nickel chloride } \\ \text { PD } & \text { Parkinson's disease } \\ \text { PHA-L } & \text { leucoagglutinin } \\ \text { PPN } & \text { peduncular pontine nucleus } \\ \text { PR } & \text { premature responses } \\ \text { PrL } & \text { prelimbic } \\ \text { PSTH } & \text { peristimulus time histogram } \\ \text { SD } & \text { standard deviation } \\ \text { SEM } & \text { standard error of mean } \\ \text { SMA } & \text { supplementary motor area } \\ \text { SN } & \text { substantia nigra } \\ \text { SNC } & \text { substantia nigra compacta } \\ \text { SNr } & \text { substantia nigra reticulata } \\ \text { STN } & \text { subthalamic nucleus } \\ \text { TBS } & \text { tris-buffered saline } \\ \text { TBS-T } & \text { tris-buffered saline -Triton } \\ \text { TMS } & \text { transcranial magnetic stimulation } \\ \text { TH } & \text { tyrosine hydroxylase } \\ \text { THir } & \text { tyrosine hydroxylase immunoreactive } \\ \text { UPDRS } & \text { unified Parkinson's disease rating scale } \\ \text { VD } & \text { ventrodorsal } \\ \text { VmPFC } & \text { ventromedial prefrontal cortex } \\ \text { WGA-HRP } & \text { wheat germ agglutinin-horseradish peroxidase } \\ \text { 5-HT } & \text { 5-hydroxytryptamine; serotonin } \\ \text { 6-OHDA } & \text { 6-hydroxy-dopamine } \\ & \end{array}$




\title{
General introduction
}

\author{
Parkinson's disease, the subthalamic \\ nucleus and deep brain stimulation.
}





\section{Parkinson's disease}

Parkinson disease (PD) is a prevalent progressive neurodegenerative disorder with a major impact on the quality of life of patients and their families (Albin, et al., 1989, Wichmann and DeLong, 1996). In addition, due to the demographic changes the prevalence is increasing and leading to a larger socio-economic burden. In Europe 108 out of 100.000 people suffer from PD (Virginia, 2008). The key motor symptoms are tremor, rigidity, bradykinesia and postural instability (Blandini, et al., 2000, Haegelen, et al., 2009). Besides the motor symptoms, PD patients also suffer from non-motor symptoms such as cognitive impairments and mood changes. PD was recognized in 1817 when the British physician James Parkinson published his essay on the 'shaking palsy (Parkinson, 1817). Loss of neuromelanin-containing dopamine (DA) cells in the substantia nigra pars compacta (SNc) is one of the main neuropathological hallmarks of the disease with the presence of Lewy bodies. Lewy bodies are abnormal aggregates of alpha-synuclein fibrils inside neurons. In the early stages of the disease motor symptoms can be adequately treated by DA replacement therapy, mainly by the dopamine precursor levodopa (L-dopa). Unfortunately, the beneficial effects wear off progressively and are replaced by disabling side effects, such as 'on-off' fluctuations and L-dopa induced dyskinesias.

\section{The subthalamic nucleus}

The subthalamic nucleus (STN) plays an important role in the pathophysiology of PD. The STN was first described by Jules Bernard Luys in 1865, probably unaware of the description of the lentiform nucleus provided by Karl Friedrich Burdach more than 40 years earlier. Luys named the nucleus "Bandelette accessoire des olives supérieur" in his first book entitled "Recherches sur le système cérébro-spinal, sa structure, ses fonctions et ses maladies" (Luys, 1865). In 1877, despite the incorrect denomination used by Luys, the STN was named by August Forel 'corpus Luysii', after its discoverer. In the current literature the STN is still often referred to as corpus Luysii (Hameleers, et al., 2006, Parent, 2002).

The STN is a spindle-shaped nucleus, located in the diencephalo-mesencephalic junction. It lies ventral to the thalamus and dorsolateral to the substantia nigra (SN) and lies within a hollow of the cerebral peduncle covered by a thin layer of fibers, the fields of Forel. The rat STN is considered to be an open nucleus, which means that the dendrites of the STN neurons reach outside the boundaries of the STN, while in humans the nucleus is closed (except for the medial border). The STN distinguishes itself from all other, inhibitory, basal ganglia nuclei since it expresses the excitatory neurotransmitter glutamate (Smith and Parent, 1988). 
STN neurons receive major input from the cortex and globus pallidus externus (GPe) and are under influence of the monoaminergic systems: fibers from the SNc, dorsal raphe nucleus (DRN) and the locus coeruleus (LC) make synaptic contact in the STN both pre- and post-synaptically releasing respectively DA, serotonin (5-HT) and noradrenalin (NA) (Boyajian, et al., 1987, Canteras, et al., 1990, Steinbusch, 1981). Within the cortico-basal ganglia thalamo-cortical circuit, the STN plays an important role in not only motor, but also in cognitive and limbic processes. Classically the STN is divided into three subregions: a motor, limbic and associative part (Hamani, et al., 2004, Temel, et al., 2005).

The STN neuronal firing properties have been studied in detail. STN neurons can express three different firing patterns: regular, irregular or bursty firing. Under normal conditions STN neurons show a mainly regular firing pattern around $20 \mathrm{~Hz}$ (Wichmann, et al., 1994). The discovery of the 1-methyl-4pheny-1.2.3.6-tetrahydropyridine (MPTP) non-human primate animal model of PD shed new light on the pathophysiological mechanisms of the disease. An important discovery was that in this animal model of PD the STN neurons were hyperactive and showed bursty firing properties (Bergman, et al., 1994, Miller and DeLong, 1987), which was later also found in PD patients (Benazzouz, et al., 2002).

\section{The cortico-subthalamic pathway}

The classical model of the basal ganglia circuitry for motor control consists of the direct and indirect pathways (Albin, et al., 1989, Alexander and Crutcher, 1990). In this model cortical information enters the basal ganglia via the striatum and is processed via two separate pathways before the information is returned to the cortex via the thalamus. In physiological conditions, the indirect pathway is counterbalanced by the direct pathway. The indirect pathway is a multisynaptic projection from the D2 projection neurons of the striatum to the output nuclei of the basal ganglia, which inhibits movements. The indirect pathway consists of an inhibitory projection from the D2 projection neurons of the striatum to the globus pallides externus (GPe), followed by an inhibitory projection from the GPe to the STN; and is completed by a glutamatergic projection from the STN to the globus pallidus internus (GPi) and substantia nigra pars reticulata (SNr). The direct pathway is a monosynaptic inhibitory projection from the D1 projection neurons of the striatum to the basal ganglia output nuclei, SNr and GPi, which facilitates movement (DeLong, 1990). The theoretical model of the direct and indirect basal ganglia pathways has been widely used in the literature to explain the pathophysiology of movement disorders and to develop new therapeutic approaches for these disorders, such as PD (DeLong, 1990). Parkinsonian motor symptoms can be alleviated by both STN inactivation and ablation (Aziz, et al., 1991, Bergman, et al., 1990) and high frequency electrical 
stimulation, rodent models of PD (Darbaky, et al., 2003), in MPTP-treated monkeys (Benazzouz, et al., 1996, Benazzouz, et al., 1993) and in Parkinsonian patients (Krack, et al., 2003, Limousin, et al., 1995).

During the last decade, another pathway within the cortico-basal ganglia information flow regained more interest: the monosynaptic cortico-subthalamic pathway, also known as the 'hyperdirect' pathway (Nambu, et al., 2002). The functional role of this pathway in movement control was reintroduced in a theoretical model of the basal ganglia circuitry in which, in contrast to the classical model, three pathways were described, namely the direct, indirect and the 'hyperdirect' pathway (Nambu, et al., 2002). Thus the classical concept is changed; there are two main input structures of the basal ganglia: striatum and STN. Several anatomical and electrophysiological studies have been performed on the monosynaptic cortico-subthalamic ('hyperdirect') pathway, but its functional role within the cortico-basal ganglia-thalamocortical circuit is still poorly understood: what is its role in movement control? Is the cortico-subthalamic pathway signaling altered in movement disorders?

The cortico-basal ganglia-thalamo-cortical circuit does not only play an important role in the control of movements, but is also involved in cognitive and limbic processes. Currently, two major theories for cortico-basal ganglia information processing for motor, associative and limbic information exist. The first supports a parallel flow of motor, associative and limbic information through the cortico-basal ganglia circuits (Alexander and Crutcher, 1990, Alexander, et al., 1986, Groenewegen and Berendse, 1990, Volkmann, et al., 2010) and the second supports convergence of these pathways (Percheron and Filion, 1991, Percheron, et al., 1984), while others consider parallel pathways with interaction between them (Joel and Weiner, 1994). In the literature these two theories are still under debate. In the same way, the question whether the monosynaptic cortico-subthalamic pathways are organized in a strictly parallel or convergent manner is still not clearly answered.

\section{Deep brain stimulation}

Motor disturbances in MPTP treated non-human primates have been shown to be alleviated by both pharmacological inactivation and ablation of the STN (Aziz, et al., 1991, Bergman, et al., 1990). Later, these lesions were replaced by high frequency electrical stimulation of the STN (Benazzouz, et al., 1996, Benazzouz, et al., 1993). Shortly after the promising results in the MPTP-treated primates, Benabid and coworkers performed the first STN deep brain stimulation (DBS) in a PD patient (Pollak, et al., 1993). Since the introduction of STN DBS in 1993, this therapy has proven to be effective on the short and long term for the cardinal motor symptoms in patients with advanced PD (Krack, et al., 2003, Limousin, et al., 1995, Visser-Vandewalle, et al., 2005). Therefore STN DBS 
currently is the first surgical therapy of choice for PD patients who became unresponsive to conservative therapy or suffer from L-dopa induced side effects. In advanced stages of PD gait problems occur, which leads to frequent falls. Current STN DBS treatment has no effect on these axial motor symptoms.

\section{Current problem}

Unfortunately, STN DBS has not only positive effects on the motor symptoms, but also affects mood and cognition in a substantial number of patients (Berney, et al., 2002, Piasecki and Jefferson, 2004, Saint-Cyr, et al., 2000, Smeding, et al., 2006, Temel, et al., 2006, Witt, et al., 2008). These unwanted psychiatric side effects may overshadow the positive effects on the motor symptoms and become a major burden for both the patient and relatives. The challenge is to obtain good therapeutic effects and to prevent the occurrence of undesired psychiatric side effects. The undesired psychiatric side effects are thought to be caused by stimulation of the non-motor parts of the STN (Temel, et al., 2005). These psychiatric effects are associated with alterations in the serotonergic system, since high frequency stimulation (HFS) of the STN inhibited dorsal raphe nucleus (DRN) neuronal firing and a reduced release of serotonin (5hydroxytryptamine, 5-HT) in the rat (Navailles, et al., 2010, Tan, et al., 2010, Temel, et al., 2007). Another challenge is to treat the axial motor symptoms.

\section{Aim of the present study}

In this thesis we test the feasibility of reducing psychiatric side effects of STN DBS. Therefore, we conducted a set of experimental and clinical studies to investigate the mechanisms of how STN stimulation can be improved.

In chapter 1 we give insight to the reader about the outcome of STN DBS on the long term on motor disabilities and neuropsychological outcome in our own series. In chapter 2 we show that STN can produce psychiatric side effects. In this study we show also underlying functional neuroanatomical mechanisms for these side effects. In chapter 3 we present the results of a systematic review on the motor and non-motor territories of the STN and its connections. In chapter 4 we investigate how functional the nonmotor connections of the STN are, utilizing animal models. In chapter 5 we challenged the hypothesis that motor and non-motor STN subterritories are separated. We provide evidence that motor and non-motor processes are incorporated in the STN. In chapter 6 and 7 we attempt to perform selective targeting of the motor STN using the information obtained in previous chapters. Next, in chapter 8, we tested a novel technique to assess gait in a bilateral 6-OHDA model of PD. Eventually, in chapter 9 we questioned the relevance of STN burst activity in relation to motor and non-motor symptoms and ad- 
dressed this question in an experimental study. Finally we provide an overall discussion on what has been learned thus far on the motor and non-motor functions of the STN and what the next steps should be. 


\section{References}

1. Albin, R. L., Young, A. B., and Penney, J. B., 1989. The functional anatomy of basal ganglia disorders. Trends Neurosci 12, 366-375.

2. Alexander, G. E., and Crutcher, M. D., 1990. Functional architecture of basal ganglia circuits: neural substrates of parallel processing. Trends Neurosci 13, 266-271.

3. Alexander, G. E., DeLong, M. R., and Strick, P. L., 1986. Parallel organization of functionally segregated circuits linking basal ganglia and cortex. Annu Rev Neurosci 9, 357-381.

4. Aziz, T. Z., Peggs, D., Sambrook, M. A., and Crossman, A. R., 1991. Lesion of the subthalamic nucleus for the alleviation of 1-methyl-4-phenyl-1,2,3,6-tetrahydropyridine (MPTP)-induced parkinsonism in the primate. Mov Disord 6, 288-292.

5. Benazzouz, A., Boraud, T., Feger, J., Burbaud, P., Bioulac, B., and Gross, C., 1996. Alleviation of experimental hemiparkinsonism by high-frequency stimulation of the subthalamic nucleus in primates: a comparison with L-Dopa treatment. Movement disorders : official journal of the Movement Disorder Society 11, 627-632.

6. Benazzouz, A., Breit, S., Koudsie, A., Pollak, P., Krack, P., and Benabid, A. L., 2002. Intraoperative microrecordings of the subthalamic nucleus in Parkinson's disease. Mov Disord 17 Suppl 3, S145-149.

7. Benazzouz, A., Gross, C., Feger, J., Boraud, T., and Bioulac, B., 1993. Reversal of rigidity and improvement in motor performance by subthalamic high-frequency stimulation in MPTP-treated monkeys. Eur J Neurosci 5, 382-389.

8. Bergman, H., Wichmann, T., and DeLong, M. R., 1990. Reversal of experimental parkinsonism by lesions of the subthalamic nucleus. Science 249, 1436-1438.

9. Bergman, H., Wichmann, T., Karmon, B., and DeLong, M. R., 1994. The primate subthalamic nucleus. II. Neuronal activity in the MPTP model of parkinsonism. J Neurophysiol 72, 507-520.

10. Berney, A., Vingerhoets, F., Perrin, A., Guex, P., Villemure, J. G., Burkhard, P. R., Benkelfat, C., and Ghika, J., 2002. Effect on mood of subthalamic DBS for Parkinson's disease: a consecutive series of 24 patients. Neurology 59, 1427-1429.

11. Blandini, F., Nappi, G., Tassorelli, C., and Martignoni, E., 2000. Functional changes of the basal ganglia circuitry in Parkinson's disease. Prog Neurobiol 62, 63-88.

12. Boyajian, C. L., Loughlin, S. E., and Leslie, F. M., 1987. Anatomical evidence for alpha-2 adrenoceptor heterogeneity: differential autoradiographic distributions of $[3 \mathrm{H}]$ rauwolscine and $[3 \mathrm{H}] \mathrm{idazoxan}$ in rat brain. The Journal of pharmacology and experimental therapeutics 241, 1079-1091.

13. Canteras, N. S., Shammah-Lagnado, S. J., Silva, B. A., and Ricardo, J. A., 1990. Afferent connections of the subthalamic nucleus: a combined retrograde and anterograde horseradish peroxidase study in the rat. Brain Res 513, 43-59.

14. Darbaky, Y., Forni, C., Amalric, M., and Baunez, C., 2003. High frequency stimulation of the subthalamic nucleus has beneficial antiparkinsonian effects on motor functions in rats, but less efficiency in a choice reaction time task. Eur J Neurosci 18, 951-956.

15. Delong, M. R., 1990. Primate models of movement disorders of basal ganglia origin. Trends Neurosci 13, 281-285.

16. Groenewegen, H. J., and Berendse, H. W., 1990. Connections of the subthalamic nucleus with ventral striatopallidal parts of the basal ganglia in the rat. J Comp Neurol 294, 607-622.

17. Haegelen, C., Rouaud, T., Darnault, P., and Morandi, X., 2009. The subthalamic nucleus is a key-structure of limbic basal ganglia functions. Med Hypotheses 72, 421-426.

18. Hamani, C., Saint-Cyr, J. A., Fraser, J., Kaplitt, M., and Lozano, A. M., 2004. The subthalamic nucleus in the context of movement disorders. Brain 127, 4-20.

19. Hameleers, R., Temel, Y., and Visser-Vandewalle, V., 2006. History of the corpus luysii: 1865-1995. Arch Neurol 63, 1340-1342.

20. Joel, D., and Weiner, I., 1994. The organization of the basal ganglia-thalamocortical circuits: open interconnected rather than closed segregated. Neuroscience 63, 363-379. 
21. Krack, P., Batir, A., Van Blercom, N., Chabardes, S., Fraix, V., Ardouin, C., Koudsie, A., Limousin, P. D., Benazzouz, A., LeBas, J. F., Benabid, A. L., and Pollak, P., 2003. Five-year follow-up of bilateral stimulation of the subthalamic nucleus in advanced Parkinson's disease. N Engl J Med 349, 1925-1934.

22. Limousin, P., Pollak, P., Benazzouz, A., Hoffmann, D., Broussolle, E., Perret, J. E., and Benabid, A. L., 1995. Bilateral subthalamic nucleus stimulation for severe Parkinson's disease. Mov Disord 10, 672-674.

23. Luys, J., 1865. Recherches sur le système cérébro-spinal, sa structure, ses fonctions et ses maladies. . Paris: Baillière.

24. Miller, W. C., and DeLong, M. R., 1987. Altered tonic activity of neurons in the globus pallidus and subthalamic nucleus in the primate model of parkinsonism. In: Carpenter, M. B., and Jayaraman, A., (Eds.), The Basal Ganglia II: structure and function. Plenum, New York, pp. 415-427.

25. Nambu, A., Tokuno, H., and Takada, M., 2002. Functional significance of the cortico-subthalamo-pallidal 'hyperdirect' pathway. Neurosci Res 43, 111-117.

26. Navailles, S., Benazzouz, A., Bioulac, B., Gross, C., and De Deurwaerdere, P., 2010. High-frequency stimulation of the subthalamic nucleus and L-3,4-dihydroxyphenylalanine inhibit in vivo serotonin release in the prefrontal cortex and hippocampus in a rat model of Parkinson's disease. J Neurosci 30, 2356-2364.

27. Parent, A., 2002. Jules Bernard Luys and the subthalamic nucleus. Movement disorders 17, 181-185.

28. Parkinson, J., 1817. An Essay on the Shaking Palsy. Sherwood, Neely, and Jones, London.

29. Percheron, G., and Filion, M., 1991. Parallel processing in the basal ganglia: up to a point. Trends Neurosci 14, 55-59.

30. Percheron, G., Yelnik, J., and Francois, C., 1984. A Golgi analysis of the primate globus pallidus. III. Spatial organization of the striato-pallidal complex. J Comp Neurol 227, 214-227.

31. Piasecki, S. D., and Jefferson, J. W., 2004. Psychiatric complications of deep brain stimulation for Parkinson's disease. J Clin Psychiatry 65, 845-849.

32. Pollak, P., Benabid, A. L., Gross, C., Gao, D. M., Laurent, A., Benazzouz, A., Hoffmann, D., Gentil, M., and Perret, J., 1993. [Effects of the stimulation of the subthalamic nucleus in Parkinson disease]. Rev Neurol (Paris) 149, 175-176.

33. Saint-Cyr, J. A., Trepanier, L. L., Kumar, R., Lozano, A. M., and Lang, A. E., 2000. Neuropsychological consequences of chronic bilateral stimulation of the subthalamic nucleus in Parkinson's disease. Brain 123 ( Pt 10), 2091-2108.

34. Smeding, H. M., Speelman, J. D., Koning-Haanstra, M., Schuurman, P. R., Nijssen, P., van Laar, T., and Schmand, B., 2006. Neuropsychological effects of bilateral STN stimulation in Parkinson disease: a controlled study. Neurology 66, 1830-1836.

35. Smith, Y., and Parent, A., 1988. Neurons of the subthalamic nucleus in primates display glutamate but not GABA immunoreactivity. Brain Res 453, 353-356.

36. Steinbusch, H. W., 1981. Distribution of serotonin-immunoreactivity in the central nervous system of the rat-cell bodies and terminals. Neuroscience 6, 557-618.

37. Tan, S. K. H., Hartung, H., Temel, Y., and Sharp, T., High frequency stimulation of the subthalamic nucleus inhibts the firing of juxtacellular labelled 5-HT-containing neurons and decreases 5-HT release in vivo., Society for Neuroscience, San Diego, 2010.

38. Temel, Y., Blokland, A., Steinbusch, H. W., and Visser-Vandewalle, V., 2005. The functional role of the subthalamic nucleus in cognitive and limbic circuits. Prog Neurobiol 76, 393-413.

39. Temel, Y., Boothman, L. J., Blokland, A., Magill, P. J., Steinbusch, H. W., Visser-Vandewalle, V., and Sharp, T., 2007. Inhibition of 5-HT neuron activity and induction of depressive-like behavior by high-frequency stimulation of the subthalamic nucleus. Proc Natl Acad Sci U S A 104, 17087-17092.

40. Temel, Y., Kessels, A., Tan, S., Topdag, A., Boon, P., and Visser-Vandewalle, V., 2006. Behavioural changes after bilateral subthalamic stimulation in advanced Parkinson disease: a systematic review. Parkinsonism Relat Disord 12, 265-272.

41. Virginia, U. O., 2008. Nervous system disorders. 
42. Visser-Vandewalle, V., van der Linden, C., Temel, Y., Celik, H., Ackermans, L., Spincemaille, G., and Caemaert, J., 2005. Long-term effects of bilateral subthalamic nucleus stimulation in advanced Parkinson disease: a four year follow-up study. Parkinsonism Relat Disord 11, 157-165.

43. Volkmann, J., Daniels, C., and Witt, K., 2010. Neuropsychiatric effects of subthalamic neurostimulation in Parkinson disease. Nature reviews. Neurology 6, 487-498.

44. Wichmann, T., Bergman, H., and DeLong, M. R., 1994. The primate subthalamic nucleus. I. Functional properties in intact animals. J Neurophysiol 72, 494-506.

45. Wichmann, T., and DeLong, M. R., 1996. Functional and pathophysiological models of the basal ganglia. Curr Opin Neurobiol 6, 751-758.

46. Witt, K., Daniels, C., Reiff, J., Krack, P., Volkmann, J., Pinsker, M. O., Krause, M., Tronnier, V., Kloss, M., Schnitzler, A., Wojtecki, L., Botzel, K., Danek, A., Hilker, R., Sturm, V., Kupsch, A., Karner, E., and Deuschl, G., 2008. Neuropsychological and psychiatric changes after deep brain stimulation for Parkinson's disease: a randomised, multicentre study. Lancet Neurol 7, 605-614. 


\section{CHAPTER 1}

\section{Subthalamic nucleus high frequency stimulation for advanced Parkinson's disease:}

motor and neuropsychological outcome after 10 years.

Janssen MLF, Duits AA, Turaihi AH, Ackermans L, Leentjens AFG,

Van Kranen-Mastenbroek V, Oosterloo M, Visser-Vandewalle $V$, Temel Y. 


\section{Abstract}

Background: Since the introduction of STN DBS many clinical studies have shown that this therapy is safe and effective on short- and mid-term. Only little is known about the long term follow-up.

Objectives: Analysis of motor and cognitive outcome during 10 years follow-up after STN DBS.

Methods: In this observational cohort study, we report on the motor and cognitive outcome in a cohort of 26 PD patients, who were prospectively followed up to 10 years after STN DBS surgery.

Results: In the early post-operative phase improvement on the UPDRS III (10.6, $p<0.01)$ and IV $(2.5, p<0.01)$ was seen and a $32 \%$ reduction in LED $(p<0.01)$. After five years a worsening of the motor performance was observed. The worsening in motor performance was mainly due to a deterioration of bradykinesia $(12.4 \pm 4.6, p<0.05)$ and axial symptoms $(6.9 \pm 2.8, \mathrm{p}<0.01)$. Memory function seemed to improve on short term, but there was a significant decline between 1 and 5 years after surgery $(p<0.01)$. The course of mood remained relatively stable during follow-up and one third of the patients showed impulsive behavior after surgery.

Conclusions: The motor performance of patients showed deterioration in time, due to an increase in bradykinetic and axial symptoms. 


\section{Introduction}

The introduction of subthalamic nucleus deep brain stimulation (STN DBS) for Parkinson's disease (PD) was a major step forward in the treatment of patients in the advanced stage of the disease (Benabid, et al., 1994). Since the introduction of STN DBS in 1993 many clinical studies have shown that this therapy is safe and effective (Deuschl, et al., 2006, Romito, et al., 2002) during a four to eight year follow up (Fasano, et al., 2010, Gervais-Bernard, et al., 2009, Krack, et al., 2003, Rodriguez-Oroz, et al., 2004, Schupbach, et al., 2005, Visser-Vandewalle, et al., 2005). However, from the studies with a longer follow-up we have learned that the motor benefit slowly wears off because of disease progression with axial and non-motor symptoms, which do respond sufficiently to dopaminergic medication and stimulation. Only few studies reported a long term follow-up with data up to ten years after surgery (Castrioto, et al., 2011, Fasano, et al., 2010, Zangaglia, et al., 2012, Zibetti, et al., 2011). Their results indicated a persistent effect of stimulation on the core motor symptoms but an increase in disability and cognitive impairment due to disease progression. Given the impact of disease progression on outcome, STN DBS is more and more considered in patients with early motor complications as a combined treatment with levodopa (Schuepbach, et al., 2013). We started our STN DBS program for PD patients in 1999. In this observational cohort study, we report on the motor and cognitive outcome in a cohort of 26 PD patients, who were prospectively followed up to 10 years after STN DBS surgery.

\section{Material and Methods}

\section{Patient population}

Between 1999 and 2003 patients with the clinical findings consistent with idiopathic PD and who suffered from severe response fluctuations and/or dyskinesias, despite optimal drug treatment, were selected for STN DBS surgery. Patients suffering from severe psychiatric co-morbidities and cognitive decline, e.g. psychosis and dementia, were excluded. Other exclusion criteria were a Hoehn and Yahr stage of five at the best moment of the day, and general contraindications for surgery such as severe hypertension or blood coagulation disorders. For more details of the in- and exclusion criteria, see previous publications (Kocabicak and Temel, 2013, Temel, et al., 2007).

\section{Surgery}

The stereotactic procedure was performed under local anesthesia. A stereotactic frame (CRW, Radionics, or Leksell G, Elekta) was fixed on the skull of the patient. A CT-scan of the head with frame was fused with a pre-operative MRI ( $1 \mathrm{~mm}$ axial T1W, and $2 \mathrm{~mm}$ T2W images) using NeuroPlan (Radionics, Ghent, Belgium) and laterthe FrameLinkTM 
software (Medtronic, Minneapolis, USA). The target was defined on the fused CT-MRI images by using the combination of atlas-based indirect coordinates (11-12 mm lateral to the AC-PC line, $2 \mathrm{~mm}$ posterior to the mid-commissural point, and $4 \mathrm{~mm}$ inferior to the intercommissural line) and MR-based direct targeting. Electrophysiologic recordings were performed in $1 \mathrm{~mm}$ steps from $10 \mathrm{~mm}$ above target, and in $0.5 \mathrm{~mm}$ steps from 5 $\mathrm{mm}$ above target, until typical nigral activity was seen. Then, test stimulation was performed. After determining the final optimal target for stimulation, a permanent quadripolar DBS macroelectrode was implanted with pole 1 at the determined optimal position within the STN (Medtronic, Minneapolis, USA, model 3389). Two to four days later, the internalisation session took place and the internal pulse generator (Kinetra and later Activa PC, Medtronic, Minneapolis, USA) was implanted. For more details of the surgical procedure, see previous publications (Kocabicak and Temel, 2013, Temel, et al., 2007).

\section{Clinical evaluation}

Patients were evaluated pre-operatively and at 3 months, 1 year, 5 years and 10 years after surgery. Post-surgical testing was conducted whilst the patients were ON medication and ON stimulation with DBS parameters that were individually optimized. Nonmotor and motor activities of daily living $(A D L)$ were evaluated using respectively Unified Parkinson's Disease Rating Scale (UPDRS) I and II. The motor performance was assessed by the UPDRS III. UPDRS III subscores were calculated for tremor, rigidity, bradykinesia and axial symptoms. The levodopa challenge on UPDRS III was calculated (Esselink, et al., 2004). Medication intake was defined by the levodopa equivalent dose (LED) (Esselink, et al., 2004). Cognition was evaluated using a battery with standardized tests assessing executive function, memory and global cognition. For the present study the following executive tests were included: the Stroop Color Word Test and the Controlled Oral Word Association Test including semantic fluency (animals and professions) and letter fluency (N, A, K). For memory, the California Verbal Learning Test (CVLT) was used and for global cognition, the Mini Mental State Examination (MMSE). See for all tests Lezak et al (Lezak, et al., 2004). The existence and severity of depressive symptoms were assessed through the Dutch version of the Beck Depression Inventory (2nd edition) (Beck, et al., 1996). Behavioral changes reported by patients or family were collected and documented. Patients gave consent for anonymous use of the collected data.

\section{Data analysis}

First, baseline characteristics were compared between patients who completed the total follow-up ( $n=12)$ and those lost during the 10 year follow-up $(n=14)$ using an independent samples t-test. A p-value of less than 0.05 was considered to be statistically significant. Non-parametric tests were used for the continuous variables. Given the 
small sample sizes of the variables, the exact Friedman's test for multiple comparisons was used to evaluate the course, with planned (between two consecutive points in time) exact Wilcoxon sign rank tests in case of significant effects. A p-value of less than 0.05 was considered to be statistically significant. No Bonferroni correction was applied to preserve statistical power and to reduce the risk of type II error. All statistical analyses were conducted using the Statistical Package for the Social Sciences version 21.0 software (SPSS Inc, Chicago, Illinois, USA).

\section{Results}

We operated on twenty-seven patients more than 10 years ago. One patient was excluded in the analyses since the diagnosis was postoperatively revised to multi system atrophy (MSA). Twelve patients completed the 10 year follow-up, but only 10 were able to perform the cognitive test battery (see Table 1 for baseline patient characteristics). Three patients died before the 5-year follow-up was reached and four were lost to follow-up due to moving to another part of the country, problems to travel to the hospital because of severe deterioration or co-morbidity. Another 7 patients died before the 10 year follow-up was reached. At baseline, patients who completed the total follow-up were younger than those who did not, respectively $54( \pm 5.8)$ and $60( \pm 6.2)$ years old, $(F$ $0.158, p=0.02$ ). No differences were found for gender, disease duration, UPDRS III (levodopa challenge), side of onset, year of operation, cognitive function and education level.

Table 1: Baseline characteristics of the patients

\begin{tabular}{ll}
\hline Gender & 18 \\
Male & 8 \\
Female & \\
Age at surgery (years) & $58.0 \pm 6.9$ \\
Mean \pm SD & $43-70$ \\
Range & \\
Disease duration (years) & $12.7 \pm 5.1$ \\
Mean $\pm S D$ & $1-20$ \\
Range & \\
Follow-up (months) & $89.6 \pm 40.6$ \\
Mean $\pm S D$ & $14-137$ \\
Range &
\end{tabular}

Description of the baseline characteristics for patients who completed the 10 year follow-up compared to patients who did not. Note that only age at time of surgery was significantly lower in patients who completed the 10 year follow-up. Values are expressed as means $\pm S D$. ${ }^{*} p<0.05$ 
Activities of daily living (ADL)

The course of the ADL is shown in table 2. Initially the UPDRS-II score improved after surgery. After 5 years however, there was a decrease in ADL function as indicated by an increase of the UPDRS-II score at 5 and 10 years after surgery. The level of functioning after 10 years with DBS and medication became at the level of the preoperative medication OFF score.

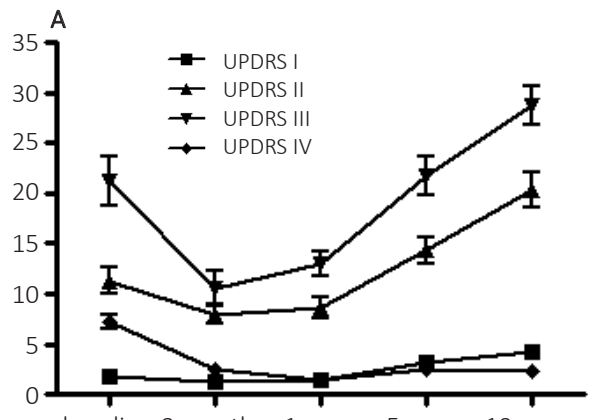

baseline 3 months 1 year 5 years 10 years

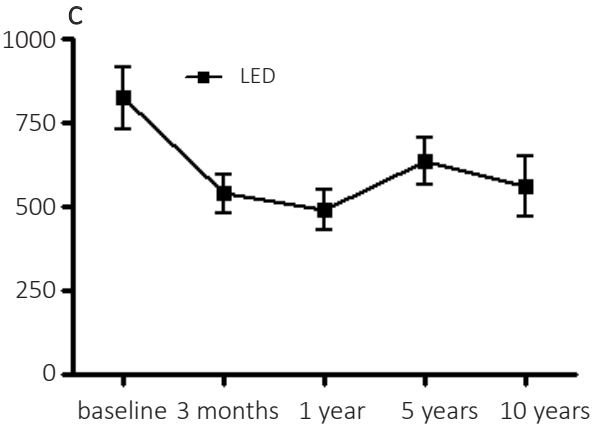

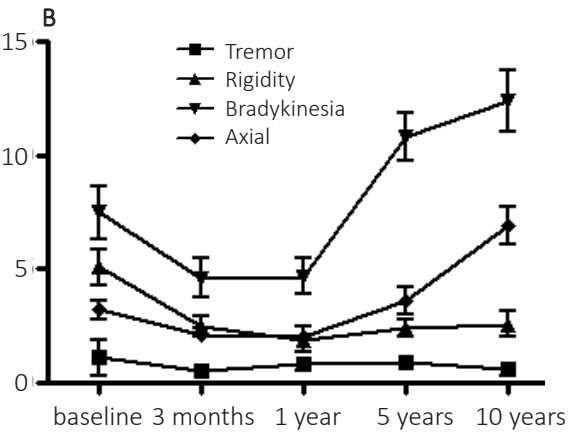

Fig. 1. UPDRS (sub-)scores and LED changes over time. Patients were scored postoperatively with stimulation and medication ON. In the graph means are plotted with an error bar representing the standard deviation. Baseline values plotted are with medication ON. (A) Postoperatively, UPDRS values initially improved. After 5 years, UPDRS III scores are at the same level as baseline and further increases at 10 years follow-up. (B) The increase in UPDRS III score was mainly due to an increase of bradykinesia and axial symptoms. (C) A sustained reduction in LED was seen after surgery. ${ }^{*} p<0.05$ compared to baseline; ${ }^{*} p<0.01$ compared to baseline. UPDRS = Unified Parkinson Disease Rating Scale; LED = Levodopa Equivalent Dose.

\section{Motor outcome}

The UPDRS III score showed a clear reduction shortly after surgery. From 1 year postoperatively till 10 year, a gradual increase in the part III score was observed, mainly due to a substantial increase in bradykinesia subscores and axial symptoms subscores. The differences of the UPDRS III between 1 and 5 years and 5 and 10 years were significantly higher for the axial and bradykinesia subscores than rigidity and tremor $(p<0.05)$. Nevertheless, the part III score with stimulation and medication was still better preoperative medication OFF score $(p<0.05)$. 
The LED was reduced immediately after surgery with $32 \%$ and at one year follow-up with $42 \%$ compared to baseline and this LED reduction sustained until 10 years of follow-up (see table 2). The improvement in the part IV score remained stable over the years, in line with the LED reduction.

Table 2: Outcome scores on the UPDRS and medication

\begin{tabular}{|c|c|c|c|c|c|c|}
\hline & \multicolumn{2}{|l|}{ baseline } & \multirow[t]{2}{*}{3 months } & \multirow[t]{2}{*}{1 year } & \multirow[t]{2}{*}{5 years } & \multirow[t]{2}{*}{10 years } \\
\hline & ON med & OFF med & & & & \\
\hline UPDRS I & $1.8 \pm 1.5$ & $2.7 \pm 2.2$ & $1.3 \pm 1.2^{\dagger}$ & $1.4 \pm 1.4$ & $3.1 \pm 1.3^{\# \#^{* *}}$ & $4.2 \pm 1.8^{\# \#}$ \\
\hline UPDRS II & $11.3 \pm 6.9$ & $21.6 \pm 6.8$ & $7.9 \pm 4.1^{\ddagger}$ & $8.6 \pm 5.2^{\# \#}$ & $14.3 \pm 5.4^{\# \pm * *}$ & $20.4 \pm 6.1^{\# \#^{* *}}$ \\
\hline UPDRS III & $21.2 \pm 12.7$ & $40.3 \pm 13.8$ & $10.6 \pm 8.2^{\# \# \neq}$ & $13.0 \pm 6.4^{\# \ddagger}$ & $21.7 \pm 8.2^{\ddagger^{*}}$ & $28.7 \pm 6.5^{+* *}$ \\
\hline Tremor & $1.1 \pm 2.0$ & $4.0 \pm 4.3$ & $.5 \pm 1.2$ & $.8 \pm 1.5$ & $.9 \pm 1.2$ & $.6 \pm 1.0$ \\
\hline Rigidity & $5.1 \pm 4.0$ & $8.4 \pm 5.3$ & $2.5 \pm 2.3$ & $1.9 \pm 2.9$ & $2.4 \pm 1.5$ & $2.6 \pm 2.0$ \\
\hline Bradykinesia & $\pm 7.5 \pm 5.9$ & $16.4 \pm 5.8$ & $4.6 \pm 4.4^{\# \neq}$ & $4.7 \pm 4.2^{\# \ddagger}$ & $10.8 \pm 4.4^{+* *}$ & $12.4 \pm 4.6^{\#+*}$ \\
\hline Axial & $3.2 \pm 2.1$ & $7.4 \pm 2.8$ & $2.1 \pm 1.5^{\# \neq}$ & $2.0 \pm 1.3^{\ddagger}$ & $3.6 \pm 2.5^{+*}$ & $6.9 \pm 2.8^{\# \# * *}$ \\
\hline UPDRS IV & $7.2 \pm 3.8$ & $6.7 \pm 4.2$ & $2.5 \pm 2.6^{\#+}$ & $1.5 \pm 1.9^{\#+}$ & $2.4 \pm 2.0^{\#+}$ & $2.3 \pm 1.8^{\# \#}$ \\
\hline \multirow[t]{2}{*}{ LED } & \multicolumn{2}{|c|}{$.824 \pm 479$} & $.539 \pm 298^{\# \#}$ & $.490 \pm 298^{\# \#}$ & $.636 \pm 304$ & $.562 \pm 314$ \\
\hline & \multicolumn{2}{|r|}{$n=26$} & $n=26$ & $n=26$ & $n=18$ & $n=12$ \\
\hline
\end{tabular}

UPDRS (sub-)scores and LED are shown. Patients were scored postoperatively with stimulation and medication ON. Values are expressed as means \pm SD. \#p<0.05 compared to baseline ON medication; \#\# $p<0.01$ compared to baseline ON medication; $\dagger p<0.05$ compared to baseline OFF medication; $¥ p<0.01$ compared to baseline OFF medication; * $p<0.05$ compared to timepoint before; ${ }^{* *} p<0.01$ compared to timepoint before. UPDRS = Unified Parkinson Disease Rating Scale; LED = Levodopa Equivalent Dose

Cognitive outcome, mood and behavior.

The course of the cognitive variables is shown in table 3 with significant differences between the respective time points for the time on card III of the Stroop Color-Word Test, verbal memory and verbal fluency. Post hoc Wilcoxon signed ranks tests between consecutive points in time of the time on the Stroop test and verbal fluency showed a decrease after surgery, in particular between 1 and 5 years after surgery. Memory scores showed an increase until 1 year after surgery. However, from 1 to 5 years after surgery, scores on both learning and free recall showed a decrease with further decrease of learning between 5 and 10 years. The MMSE and Beck Depression Inventory (BDI) scores showed a relatively stable course during ten years after surgery.

Based on the interviews during follow-up, 9 of the 26 (35\%) patients showed impulsive behavior. Post hoc analyses showed no significant differences between preoperative characteristics of those with and without postoperative impulsivity. There was however a trend for younger age $(p=0.06)$ in the patients with impulsivity at follow-up. No significant correlations were found between the presence of postoperatively impulsivity and performance on the Stroop test. Adjustment of parameter settings and/or 
reducing medication was helpful in most cases. Two patients showed postoperative apathy, with one patients also showing cognitive deterioration. Two patients had depressive episodes, one of them improved after adjustment of the parameters.

Table 3: Cognitive outcome and mood

\begin{tabular}{lccccc}
\hline & baseline & $\begin{array}{l}3 \text { months } \\
\text { Score }\end{array}$ & $\begin{array}{l}1 \text { year } \\
\text { Score }\end{array}$ & $\begin{array}{l}5 \text { years } \\
\text { Score }\end{array}$ & $\begin{array}{l}10 \text { years } \\
\text { Score }\end{array}$ \\
\hline Stroop Color-Word (time) & $113.4 \pm 32.5$ & $.121 \pm 41.9$ & $130.7 \pm 38.2^{*}$ & $151.6 \pm 69.1^{* *}$ & $172.9 \pm 81.8^{*}$ \\
Stroop Color-Word (error) & $.8 \pm 1.8$ & $.9 \pm 1.3$ & $1.3 \pm 2.4$ & $2.9 \pm 8.8$ & $1.4 \pm 2.1$ \\
CVLT total learned trial1-5 & $47.8 \pm 10.7$ & $48.8 \pm 9.3$ & $52.7 \pm 10.8^{* *}$ & $44.9 \pm 13^{* *}$ & $39.6 \pm 13.9^{*}$ \\
CVLT free Long term recall & $9.7 \pm 3.1$ & $10.8 \pm 3.3^{*}$ & $.011 \pm 3.3$ & $7.9 \pm 4.8^{* *}$ & $8.1 \pm 5.1$ \\
fluency categories total & $37.6 \pm 11.3$ & $34.5 \pm 9.11^{*}$ & $35.7 \pm 12.2$ & $30.1 \pm 11.1^{* *}$ & $25.4 \pm 11.7^{*}$ \\
fluency letters totaal & $36.5 \pm 13.1$ & $34.9 \pm 10.8$ & $34.7 \pm 11.2$ & $26.9 \pm 15^{*}$ & $25.4 \pm 14.7^{*}$ \\
MMSE & $28.2 \pm 3.3$ & $28.3 \pm 1.8$ & $28.6 \pm 1.9$ & $26.2 \pm 3.9$ & $25.4 \pm 5.2$ \\
BDI-II & $11.2 \pm 7.4$ & $6.7 \pm 6.8$ & $7.3 \pm 6.5$ & $8.8 \pm 5.6$ & $8.5 \pm 6.6$ \\
& $\mathrm{n}=26$ & $\mathrm{n}=26$ & $\mathrm{n}=26$ & $\mathrm{n}=14$ & $\mathrm{n}=8-10$ \\
\hline
\end{tabular}

Cognitive outcome and mood are presented. Patients were scored with medication ON and stimulation ON. Values are expressed as means \pm SD. ${ }^{*} \mathrm{p}<0.05$ compared to timepoint before; ${ }^{*} \mathrm{p}<0.01$ compared to timepoint before. CVLT = California Verbal Learning Test: MMSE = Mini Mental State Examination; BDI = Beck Depression Inventory

\section{Stimulation parameters}

At three months of follow-up the mean voltage, pulse width and frequency used were respectively for the left and right side 1.7 and $1.8 \mathrm{~V}, 84$ and $89 \mu \mathrm{s}$ and $146 \mathrm{~Hz}$. The mean voltage increased during follow-up up to respectively 3.2 and $3.7 \mathrm{~V}$ at 10 years followup. The pulse width and the frequency remained stable (see table 4).

Table 4: Stimulation parameters

\begin{tabular}{|c|c|c|c|c|c|c|c|c|}
\hline & \multicolumn{2}{|c|}{3 months follow-up } & \multicolumn{2}{|c|}{1 year follow-up } & \multicolumn{2}{|c|}{5 years follow-up } & \multicolumn{2}{|c|}{10 years follow-up } \\
\hline & Left & Right & Left & Right & Left & Right & Left & Right \\
\hline Voltage & $1.7 \pm 0.8$ & $1.8 \pm 0.9$ & $2.7 \pm 1.1^{* *}$ & $2.8 \pm 1.1^{*}$ & $3.2 \pm 1.1^{*}$ & $3.6 \pm 1.0 * *$ & $3.2 \pm 1.5$ & $3.7 \pm 1.3$ \\
\hline \multicolumn{9}{|l|}{ Pulse } \\
\hline widthW & $0.4 \pm 23$ & $.89 \pm 25$ & $.86 \pm 27$ & $.89 \pm 25$ & $.97 \pm 23$ & $.94 \pm 17$ & $.85 \pm 17$ & $.95 \pm 21$ \\
\hline Frequency & $146 \pm 42$ & & $152 \pm 42$ & & $166 \pm 23$ & & $157 \pm 26$ & \\
\hline
\end{tabular}

Stimulation parameters. Values are expressed as means \pm SD. ${ }^{*} p<0.05$ compared to timepoint before; ** $p<0.01$ compared to timepoint before. 


\section{Discussion}

In the early post-operative phase (3 months, 1 year) a strong improvement of the motor scores on the UPDRS III and IV and LED reduction was found. However, after five years a gradual worsening of the motor performance was observed, most probably due to disease progression. At ten years follow-up the UPDRS III scores were slightly worse than the pre-operative medication ON condition, but still better than the pre-operative medication OFF condition. The worsening in motor performance was almost exclusively due to a deterioration of bradykinesia and axial symptoms. In line with this, we found an increase in the UPDRS II score over time after an improvement in early post-operative phase ( 3 months, 1 year). These results are in line with earlier reports (Castrioto, et al., 2011, Krack, et al., 2003, Rodriguez-Oroz, et al., 2004, Zibetti, et al., 2011).

We have observed a considerable increase in the stimulation amplitudes over time. This has potentially two reasons. First, to counteract the worsening of the motor performance or second due to mild gliosis, which occurs along the electrode trajectory. In the patients of this study, depression scores did not change over time. It is still unclear why in some patient mood-related changes were observed after STN DBS (Voon, et al., 2008) and in others not (Castelli, et al., 2006). One third of the patients showed impulsive behavior after surgery, which appeared not to be related to response inhibition as assessed with the Stroop test. So far, however, no single cognitive test is available to capture the multifaceted construct of impulsivity (Sinha, et al., 2013).

Whereas our results seem to support the finding that STN-DBS cannot prevent cognitive decline along the course of the disease, DBS might be not as safe as suggested from a cognitive-behavioral standpoint (Zangaglia, et al., 2012). Although we did not find an association between reported behavioral changes and cognitive tests, there is evidence for an intervention related decrease in impulse control either by the surgical procedure and/or chronic stimulation. Especially younger patients seemed to be at risk of impulsive behavior, which is in line with earlier findings (Broen, et al., 2011). A decrease in performance on executive tests, on the other hand, seems to be associated with higher age and an advanced stage of the disease (Daniels, et al., 2010). Perhaps impulsive behavior is the result of a stimulation-induced combination of overestimation, increased risk taking and preference for competitive environments (Florin, et al., 2013). Therefore, optimization of the targeting of the motor part of the STN and preventing stimulation of the limbic and associative parts of the STN might help to reduce stimulation induced behavioral side effects (Janssen, et al., 2012, Maks, et al., 2009).

Today, neurologists encounter PD patients in their outpatient clinic in whom DBS was started 5 to 10 years ago. The long term combined treatment with oral drugs is not sufficient anymore in most cases and quality of life in these patients is reduced. Patients have a high risk of falling due to increased axial motor disturbances. The field is search- 
ing for therapies to specifically treat these symptoms. Surgically, DBS of the peduncular pontine nucleus (PPN) has been experimentally applied, thus far without convincing efficacy (Ferraye, et al., 2010, Stefani, et al., 2007). Also limited evidence is published on combined STN DBS and DBS of the substantia nigra pars reticulata (SNr) on gait. A small reduction in freezing of gait was found in patients who received both STN DBS and SNr DBS compared to STN DBS alone. The authors proposed that given the efferent monosynaptic GABAergic transmission from SNr to the PPN, high-frequency stimulation at the level of SNr might attenuate an overinhibitory drive (Weiss, et al., 2013). The initial clinical response to STN DBS can be predicted by the response to levo-dopa therapy at the beginning of the disease. Axial symptoms do not respond well to dopamine replacement therapies. Therefore, these symptoms are not related to the degeneration of the substantia nigra (dopaminergic system), but are more likely related to the nondopaminergic, monoaminergic systems (nor-adrenaline and serotonin) (Lang and Obeso, 2004).

This study has several limitations. Although our sample size is comparable to earlier long-term reports, only a small sample was presented. Given the consistent findings so far, the present work adds to a clearer image of the long-term results of STN DBS. A major limitation but inevitable from an ethical point of view is the lack of a control group. Other limitations in this study are non-blinded assessments and that testing was only performed in ON medication and ON stimulation conditions. The last was due to the fact that the vast majority of patients refused to be repeatedly tested in OFF conditions. Finally, we did not correct the level of significance for multiple comparisons. However, we preferred to accept a high probability of type I errors, since a high probability of type II errors might mask possible adverse effects of DBS therapy.

In conclusion, from this cohort of patients we have learned that deterioration is due to an increase in bradykinetic and axial symptoms. Nevertheless, the medication $\mathrm{ON}$ and stimulation ON motor score after 10 years is still better than the preoperative medication OFF score. Younger patients might be more at risk for impulsive behavior after surgery, but may benefit longer from DBS therapy than older patients. Since the efficacy of stimulation in earlier stages of disease has been proven to be effective (Schuepbach, et al., 2013), we think that STN DBS should be considered in patients with in early stages of disease as a combined treatment with levodopa.

\section{Acknowledgements}

This work was supported by the BrainGain Smart Mix Program of the Netherlands Ministry of Economic Affairs and the Netherlands Ministry of Education, Culture and Science [grant number SSM06011]. 


\section{References}

1. Beck, A. T., Steer, R. A., and Brown, G. K., 1996. Manual for the Beck Depression Inventory-II. Psychological Corporation, San Antonio.

2. Benabid, A. L., Pollak, P., Gross, C., Hoffmann, D., Benazzouz, A., Gao, D. M., Laurent, A., Gentil, M., and Perret, J., 1994. Acute and long-term effects of subthalamic nucleus stimulation in Parkinson's disease. Stereotact Funct Neurosurg 62, 76-84.

3. Broen, M., Duits, A., Visser-Vandewalle, V., Temel, Y., and Winogrodzka, A., 2011. Impulse control and related disorders in Parkinson's disease patients treated with bilateral subthalamic nucleus stimulation: a review. Parkinsonism Relat Disord 17, 413-417.

4. Castelli, L., Perozzo, P., Zibetti, M., Crivelli, B., Morabito, U., Lanotte, M., Cossa, F., Bergamasco, B., and Lopiano, L., 2006. Chronic deep brain stimulation of the subthalamic nucleus for Parkinson's disease: effects on cognition, mood, anxiety and personality traits. European neurology 55, 136-144.

5. Castrioto, A., Lozano, A. M., Poon, Y. Y., Lang, A. E., Fallis, M., and Moro, E., 2011. Ten-year outcome of subthalamic stimulation in Parkinson disease: a blinded evaluation. Arch Neurol 68, 1550-1556.

6. Daniels, C., Krack, P., Volkmann, J., Pinsker, M. O., Krause, M., Tronnier, V., Kloss, M., Schnitzler, A., Wojtecki, L., Botzel, K., Danek, A., Hilker, R., Sturm, V., Kupsch, A., Karner, E., Deuschl, G., and Witt, K., 2010. Risk factors for executive dysfunction after subthalamic nucleus stimulation in Parkinson's disease. Movement disorders : official journal of the Movement Disorder Society 25, 1583-1589.

7. Deuschl, G., Schade-Brittinger, C., Krack, P., Volkmann, J., Schafer, H., Botzel, K., Daniels, C., Deutschlander, A., Dillmann, U., Eisner, W., Gruber, D., Hamel, W., Herzog, J., Hilker, R., Klebe, S., Kloss, M., Koy, J., Krause, M., Kupsch, A., Lorenz, D., Lorenzl, S., Mehdorn, H. M., Moringlane, J. R., Oertel, W., Pinsker, M. O., Reichmann, H., Reuss, A., Schneider, G. H., Schnitzler, A., Steude, U., Sturm, V., Timmermann, L., Tronnier, V., Trottenberg, T., Wojtecki, L., Wolf, E., Poewe, W., and Voges, J., 2006. A randomized trial of deep-brain stimulation for Parkinson's disease. N Engl J Med 355, 896-908.

8. Esselink, R. A., de Bie, R. M., de Haan, R. J., Lenders, M. W., Nijssen, P. C., Staal, M. J., Smeding, H. M., Schuurman, P. R., Bosch, D. A., and Speelman, J. D., 2004. Unilateral pallidotomy versus bilateral subthalamic nucleus stimulation in PD: a randomized trial. Neurology 62, 201-207.

9. Fasano, A., Romito, L. M., Daniele, A., Piano, C., Zinno, M., Bentivoglio, A. R., and Albanese, A., 2010. Motor and cognitive outcome in patients with Parkinson's disease 8 years after subthalamic implants. Brain : a journal of neurology 133, 2664-2676.

10. Ferraye, M. U., Debu, B., Fraix, V., Goetz, L., Ardouin, C., Yelnik, J., Henry-Lagrange, C., Seigneuret, E., Piallat, B., Krack, P., Le Bas, J. F., Benabid, A. L., Chabardes, S., and Pollak, P., 2010. Effects of pedunculopontine nucleus area stimulation on gait disorders in Parkinson's disease. Brain : a journal of neurology 133, 205-214.

11. Florin, E., Muller, D., Pfeifer, J., Barbe, M. T., Fink, G. R., and Timmermann, L., 2013. Subthalamic stimulation modulates self-estimation of patients with Parkinson's disease and induces risk-seeking behaviour. Brain : a journal of neurology.

12. Gervais-Bernard, H., Xie-Brustolin, J., Mertens, P., Polo, G., Klinger, H., Adamec, D., Broussolle, E., and Thobois, S., 2009. Bilateral subthalamic nucleus stimulation in advanced Parkinson's disease: five year follow-up. J Neurol 256, 225-233.

13. Janssen, M. L., Zwartjes, D. G., Temel, Y., van Kranen-Mastenbroek, V., Duits, A., Bour, L. J., Veltink, P. H., Heida, T., and Visser-Vandewalle, V., 2012. Subthalamic neuronal responses to cortical stimulation. Mov Disord 27, 435-438.

14. Kocabicak, E., and Temel, Y., 2013. Deep brain stimulation of the subthalamic nucleus in Parkinson's disease: Surgical technique, tips, tricks and complications. Clinical neurology and neurosurgery.

15. Krack, P., Batir, A., Van Blercom, N., Chabardes, S., Fraix, V., Ardouin, C., Koudsie, A., Limousin, P. D., Benazzouz, A., LeBas, J. F., Benabid, A. L., and Pollak, P., 2003. Five-year follow-up of bilateral stimulation of the subthalamic nucleus in advanced Parkinson's disease. N Engl J Med 349, 1925-1934.

16. Lang, A. E., and Obeso, J. A., 2004. Challenges in Parkinson's disease: restoration of the nigrostriatal dopamine system is not enough. Lancet Neurol 3, 309-316. 
17. Lezak, M. D., Howieson, D. B., and Loring, D. W., 2004. Neuropsychological Assessment. Oxford University Press, New York.

18. Maks, C. B., Butson, C. R., Walter, B. L., Vitek, J. L., and Mclntyre, C. C., 2009. Deep brain stimulation activation volumes and their association with neurophysiological mapping and therapeutic outcomes. J Neurol Neurosurg Psychiatry 80, 659-666.

19. Rodriguez-Oroz, M. C., Zamarbide, I., Guridi, J., Palmero, M. R., and Obeso, J. A., 2004. Efficacy of deep brain stimulation of the subthalamic nucleus in Parkinson's disease 4 years after surgery: double blind and open label evaluation. J Neurol Neurosurg Psychiatry 75, 1382-1385.

20. Romito, L. M., Scerrati, M., Contarino, M. F., Bentivoglio, A. R., Tonali, P., and Albanese, A., 2002. Longterm follow up of subthalamic nucleus stimulation in Parkinson's disease. Neurology 58, 1546-1550.

21. Schuepbach, W. M., Rau, J., Knudsen, K., Volkmann, J., Krack, P., Timmermann, L., Halbig, T. D., Hesekamp, H., Navarro, S. M., Meier, N., Falk, D., Mehdorn, M., Paschen, S., Maarouf, M., Barbe, M. T., Fink, G. R., Kupsch, A., Gruber, D., Schneider, G. H., Seigneuret, E., Kistner, A., Chaynes, P., Ory-Magne, F., Brefel Courbon, C., Vesper, J., Schnitzler, A., Wojtecki, L., Houeto, J. L., Bataille, B., Maltete, D., Damier, P., Raoul, S., Sixel-Doering, F., Hellwig, D., Gharabaghi, A., Kruger, R., Pinsker, M. O., Amtage, F., Regis, J. M., Witjas, T., Thobois, S., Mertens, P., Kloss, M., Hartmann, A., Oertel, W. H., Post, B., Speelman, H., Agid, Y., Schade-Brittinger, C., and Deuschl, G., 2013. Neurostimulation for Parkinson's disease with early motor complications. N Engl J Med 368, 610-622.

22. Schupbach, W. M., Chastan, N., Welter, M. L., Houeto, J. L., Mesnage, V., Bonnet, A. M., Czernecki, V., Maltete, D., Hartmann, A., Mallet, L., Pidoux, B., Dormont, D., Navarro, S., Cornu, P., Mallet, A., and Agid, Y., 2005. Stimulation of the subthalamic nucleus in Parkinson's disease: a 5 year follow up. J Neurol Neurosurg Psychiatry 76, 1640-1644.

23. Sinha, N., Manohar, S., and Husain, M., 2013. Impulsivity and apathy in Parkinson's disease. Journal of neuropsychology 7, 255-283.

24. Stefani, A., Lozano, A. M., Peppe, A., Stanzione, P., Galati, S., Tropepi, D., Pierantozzi, M., Brusa, L., Scarnati, E., and Mazzone, P., 2007. Bilateral deep brain stimulation of the pedunculopontine and subthalamic nuclei in severe Parkinson's disease. Brain 130, 1596-1607.

25. Temel, Y., Wilbrink, P., Duits, A., Boon, P., Tromp, S., Ackermans, L., van Kranen-Mastenbroek, V., Weber, W., and Visser-Vandewalle, V., 2007. Single electrode and multiple electrode guided electrical stimulation of the subthalamic nucleus in advanced Parkinson's disease. Neurosurgery 61, 346-355; discussion 355-347.

26. Visser-Vandewalle, V., van der Linden, C., Temel, Y., Celik, H., Ackermans, L., Spincemaille, G., and Caemaert, J., 2005. Long-term effects of bilateral subthalamic nucleus stimulation in advanced Parkinson disease: a four year follow-up study. Parkinsonism Relat Disord 11, 157-165.

27. Voon, V., Krack, P., Lang, A. E., Lozano, A. M., Dujardin, K., Schupbach, M., D'Ambrosia, J., Thobois, S., Tamma, F., Herzog, J., Speelman, J. D., Samanta, J., Kubu, C., Rossignol, H., Poon, Y. Y., Saint-Cyr, J. A., Ardouin, C., and Moro, E., 2008. A multicentre study on suicide outcomes following subthalamic stimulation for Parkinson's disease. Brain 131, 2720-2728.

28. Weiss, D., Walach, M., Meisner, C., Fritz, M., Scholten, M., Breit, S., Plewnia, C., Bender, B., Gharabaghi, A., Wachter, T., and Kruger, R., 2013. Nigral stimulation for resistant axial motor impairment in Parkinson's disease? A randomized controlled trial. Brain : a journal of neurology 136, 2098-2108.

29. Zangaglia, R., Pasotti, C., Mancini, F., Servello, D., Sinforiani, E., and Pacchetti, C., 2012. Deep brain stimulation and cognition in Parkinson's disease: an eight-year follow-up study. Movement disorders : official journal of the Movement Disorder Society 27, 1192-1194.

30. Zibetti, M., Merola, A., Rizzi, L., Ricchi, V., Angrisano, S., Azzaro, C., Artusi, C. A., Arduino, N., Marchisio, A., Lanotte, M., Rizzone, M., and Lopiano, L., 2011. Beyond nine years of continuous subthalamic nucleus deep brain stimulation in Parkinson's disease. Movement disorders : official journal of the Movement Disorder Society 26, 2327-2334. 


\section{CHAPTER 2}

\section{High frequency stimulation of the subthalamic nucleus increases c-Fos immunoreactivity in the dorsal raphe nucleus and afferent brain regions}

Tan SKH, Janssen MLF, Jahanshahi A, Chouliaras L, Visser-Vandewalle V, Lim LW, Steinbusch HWM, Sharp T, Temel Y.

J Psychiatr Res. 2011 Oct;45(10):1307-15. 


\section{Abstract}

High frequency stimulation (HFS) of the subthalamic nucleus (STN) is the neurosurgical therapy of choice for the management of motor deficits in patients with advanced Parkinson's disease, but this treatment can elicit disabling mood changes. Our recent experiments show that in rats, HFS of the STN both inhibits the firing of 5-HT (5hydroxytryptamine; serotonin) neurons in the dorsal raphe nucleus (DRN) and elicits 5$\mathrm{HT}$-dependent behavioral effects. The neural circuitry underpinning these effects is unknown. Here we investigated in the dopamine-denervated rat the effect of bilateral HFS of the STN on markers of neuronal activity in the DRN as well as DRN input regions. Controls were sham-stimulated rats. HFS of the STN elicited changes in two 5-HTsensitive behavioral tests. Specifically, HFS increased immobility in the forced swim test and increased interaction in a social interaction task. HFS of the STN at the same stimulation parameters, increased c-Fos immunoreactivity in the DRN, and decreased cytochrome $\mathrm{C}$ oxidase activity in this region. The increase in c-Fos immunoreactivity occurred in DRN neurons immunopositive for the GABA marker parvalbumin. HFS of the STN also increased the number of c-Fos immunoreactive cells in the lateral habenula nucleus, medial prefrontal cortex but not significantly in the substantia nigra. Collectively, these findings support a role for circuitry involving DRN GABA neurons, as well as DRN afferents from the lateral habenula nucleus and medial prefrontal cortex, in the mood effects of HFS of the STN. 


\section{Introduction}

Currently, high frequency stimulation (HFS) of the subthalamic nucleus (STN) is the neurosurgical therapy of choice for treatment resistant patients with advanced Parkinson's disease (PD). Randomized controlled trials have shown that HFS of the STN was superior over best medical treatment (Deuschl, et al., 2006, Weaver, et al., 2009, Williams, et al., 2010). Despite improving motor disability, in some patients HFS of the STN induces mood disorders such as depression and increased impulsivity (Berney, et al., 2002, Houeto, et al., 2002, Temel, et al., 2006). In addition, evidence suggests that the risk of suicide and suicide attempts increases significantly (Soulas, et al., 2008, Voon, et al., 2008). These mood-related side-effects often mitigate the positive effects on motor symptoms and negatively influence the quality of life of patients and their families (Schrag, et al., 2000, Troster, et al., 2003).

Depression, impulsivity and suicide are associated with a dysfunctional 5hydroxytryptamine (5-HT; serotonin) system (Mann, 2003, Smith, et al., 1997). Recently we found that, in a rat model of PD, bilateral HFS of the STN inhibited the firing rate of 5-HT neurons of the dorsal raphe nucleus (DRN) and induced 5-HTdependent changes in depressive-like behavior (Hartung, et al., 2011, Temel, et al., 2007). Furthermore, in microdialysis studies HFS of the STN was reported to decrease 5-HT release in the rat forebrain (Navailles, et al., 2010), and we have observed this effect in similar studies (Tan, et al., 2010). These findings support the idea that changes in mood induced by HFS of the STN are caused by reduced 5-HT function.

The neural circuitry underpinning the effect of HFS of the STN on 5-HT neurons is not known but likely involves indirect projections from the STN to the DRN. Thus, although there is no direct projection from the STN to the DRN (Peyron, et al., 1998), projections from the STN target regions with major inputs to the DRN including the medial prefrontal cortex (MPFC), lateral habenula nucleus (LHb) and substantia nigra reticulata (SNr) and compacta (SNc) (Aghajanian and Wang, 1977, Hajos, et al., 1998, Jankowski and Sesack, 2004, Kirouac, et al., 2004, Varga, et al., 2001). $p$-aminobutyric acid (GABA) neurons in the DRN may be a key part of the circuitry because they inhibit nearby 5-HT neurons (Liu, et al., 2000) and are selectively targeted by inputs from the mPFC and LHb (Hajos, et al., 1998, Varga, et al., 2003, Varga, et al., 2001).

The present study used molecular markers of neural activity to test the hypothesis that HFS of the STN alters the function of GABA neurons in DRN, as well as DRN input regions. The principle marker used was the activity-dependent immediate early gene cFos, supplemented by measurements of the metabolic enzyme cytochrome $\mathrm{C}$ oxidase. Initial experiments established STN stimulation parameters that would evoke behavioral changes in 5-HT-dependent tests of emotionality. 


\section{Material and Methods}

\section{Animals}

Male Lewis rats (280-320 g, Maastricht University) were housed individually under conditions of constant temperature $\left(20-22{ }^{\circ} \mathrm{C}\right)$ and humidity $(60-70 \%)$ with a reversed light/dark cycle (lights on 17h-05h). Rats had access to water and food ad libitum. Experiments were ethically reviewed and approved by the Animal Experimental Committee of Maastricht University.

\section{Experimental groups}

Rats were randomly assigned to one of three groups: i) neurosurgical sham-control without STN electrode implants $(n=6)$, ii) dopamine lesion without STN electrode implants $(n=10)$ and iii) dopamine lesion with STN electrode implants $(n=12)$.

\section{Dopamine lesions and STN electrode implantation}

Rats were dopamine-denervated by bilateral intra-striatal injection of 6-hydroxydopamine (6-OHDA). Rats were pretreated ( $1 \mathrm{~h})$ with desipramine $(20 \mathrm{mg} / \mathrm{kg}$ i.p.) prior to induction and maintenance of general anesthesia using a combination of ketamine (90 mg/kg i.p.) and xylazine (10 mg/kg i.p.), and placement in a stereotactic frame (Stoelting, Wood Dale, USA; model 51653). Either 6-OHDA $(5 \mathrm{mg} / \mathrm{ml} 6-\mathrm{OHDA}$ in $0.9 \%$ saline with $0.2 \%$ ascorbic acid) or vehicle $(0.9 \%$ saline with $0.2 \%$ ascorbic acid) was injected bilaterally $(2 \mathrm{ml}$ at $0.5 \mathrm{ml} / \mathrm{min}$ ) in two striatal locations (coordinates according to bregma and skull surface AP p0.7 mm, ML +/-2.8 mm, DV -5.0 mm and AP -0.4 mm, ML +/-3.4 mm, DV -5.0 mm; (Paxinos and Watson, 1998). Bipolar stimulating electrodes were then implanted bilaterally into the STN (AP $-3.8 \mathrm{~mm}, \mathrm{ML}+/-2.5 \mathrm{~mm}$, DV $-8.0 \mathrm{~mm}$ ) and fixed in place using dental cement (Heraeus Kulzer, Germany) and skull screws, as described previously (Tan, et al., 2010). For animals in both neurosurgical sham-controls and dopamine lesion, electrodes were inserted bilaterally into the STN but then removed before closing the skull.

\section{High frequency stimulation}

Rats were allowed a minimum of 3 weeks to recover from surgery before proceeding with stimulation and behavioral testing. Bipolar stimulation parameters $(130 \mathrm{~Hz}, 60 \mathrm{~ms}$, $150 \mathrm{~mA}$ ) were clinically relevant and previously shown in animal models to evoke changes in emotional and cognitive responses, and inhibit 5-HT neuronal activity (Tan, et al., 2010, Temel, et al., 2007, Temel, et al., 2005). Stimulation was delivered via a stimulus isolator (A360, World Precision Instruments) driven by a stimulus generator (World Precision Instrument, A310 Accupulser, Germany) connected to a digital oscilloscope (Agilent 54622D oscilloscope, Agilent Technologies, the Netherlands) to monitor 
current output (Tan, et al., 2010, Temel, et al., 2004). Stimulation was delivered daily, either for the duration of the behavioral paradigm on testing days or for 30 min on nontesting days.

\section{Forced swim test}

Rats were tested in the forced swim test (FST), which is a well validated model of depressive-like behavior and sensitive to 5-HT manipulations (Cryan, et al., 2002). The FST was carried out using clear Perspex cylinders (height $50 \mathrm{~cm}$ x diameter $20 \mathrm{~cm}$ ) filled with water ( $30 \mathrm{~cm}$ deep, $25^{\circ} \mathrm{C}$ ) and surrounded by black walls. On the pretest day, rats were placed individually in a water-filled cylinder for $15 \mathrm{~min}$. This was repeated on the test day (24 h later) for $5 \mathrm{~min}$, while stimulation was applied to rats with implanted electrodes (Temel, et al., 2007). All sessions were videotaped and analyzed independently by two investigators. Immobility was defined as the time of no movement or minor movements whilst keeping the nose above the water (Van den Hove, et al., 2005).

\section{Social interaction}

Rats were also tested in a social interaction test in which increased social interaction is associated with decreased 5-HT levels (Collins, et al., 1979, Tonissaar, et al., 2008). The protocol was as previously described (File and Hyde, 1978) with modification (Kask, et al., 2001). In brief, two weight-matched rats with implanted STN electrodes were taken from their home cages and placed in a novel test environment (50 x $50 \mathrm{~cm}$ open field) for 5 min during which one of the two rats was stimulated. Sessions were recorded on video and analyzed off-line by two investigators who scored the time the stimulated rat spent sniffing, following and crawling under and over the non-stimulated rat.

\section{Histological and immunocytochemical staining}

Following the behavioral experiments, rats with STN electrodes were stimulated (30 min) on one final occasion and sacrificed. Two hours after the last stimulation rats were perfused transcardially with Tyrode's buffer $(0.1 \mathrm{M})$ and fixative containing $4 \%$ paraformaldehyde, $15 \%$ picric acid and $0.05 \%$ glutaraldehyde in $0.1 \mathrm{M}$ phosphate buffer $(\mathrm{pH}$ 7.6) at $4{ }^{\circ} \mathrm{C}$. Brains were then removed and post-fixed for $2 \mathrm{~h}$ at $4{ }^{\circ} \mathrm{C}$ prior to overnight immersion in $15 \%$ sucrose at $4{ }^{\circ} \mathrm{C}$. Finally, brains were snap-frozen in solid carbon dioxide and stored at $-80{ }^{\circ} \mathrm{C}$ before being sectioned serially $(30 \mathrm{~mm}$ ) using a cryostat. Sections were then kept at $-80^{\circ} \mathrm{C}$ before staining.

\section{Electrode localization}

Coronal sections containing the STN region and the electrode trajectory were mounted on gelatin-coated slides, and stained with a standard hematoxylin-eosin (Merck, Darm- 
stadt, Germany) histological procedure (Tan, et al., 2010) prior to evaluation of the location of the electrode tip.

\section{Tyrosine hydroxylase immunocytochemistry}

Dopaminergic lesions were verified by tyrosine hydroxylase (TH) immunocytochemistry. Sections containing the SNc were incubated overnight with the mouse anti-TH primary antibody (1:8000 dilution, kindly supplied by Dr. C. Cuello, Canada), washed with Trisbuffered saline (TBS) and TBS-Triton X-100 (TBS-T), and then incubated with the secondary antibody (donkey anti-mouse biotin, 1:800 dilution, Jackson Immunoresearch Laboratories, West Grove, USA). This was followed by $2 \mathrm{~h}$ exposure to avidinbiotinperoxidase complex (Elite ABC kit, Vectastain; Vector Laboratories, Burlingame, USA), and the horseradish peroxidase complex was visualized using a 3,3-diaminobenzidine (DAB) solution. Brain sections were mounted on gelatin-coated slides, air-dried, dehydrated and cover-slipped using Pertex (Histolab Products ab, Göteborg, Sweden). THpositive cells in the SNc were counted using stereology (CAST-GRID-Computer Assisted Stereological Toolbox; Olympus, Hamburg, Germany). After delineating the SNc using microscopic video images, cell numbers were determined using an optical fractionator (Schmitz and Hof, 2005). The area of interest was analyzed in a systematic-random fashion as described previously (Temel, et al., 2006).

\section{c-Fos immunocytochemistry and double-labeling with parvalbumin}

Brain sections were processed for c-Fos immunocytochemistry. In brief, sections were incubated overnight with polyclonal rabbit anti-c-Fos primary antibody (1:10,000; Santa Cruz Biotechnology Inc, Santa Cruz, USA) and then, after washing, biotinylated donkey anti-rabbit secondary antibody (1:800; Jackson Immunoresearch Laboratories Inc., Westgrove, USA). Visualization of the HRP product was similar to that used for TH immunostaining except with nickel enhancement. A series of sections from STN stimulated rats were examined for double-labeling of c-Fos and parvalbumin, a calcium-binding protein expressed by a subpopulation of putative GABAergic neurons (Celio, 1990, Charara and Parent, 1998). These sections were incubated with the primary antibodies, rabbit anti-c-Fos (1:5000; Santa Cruz Biotechnology Inc, Santa Cruz, USA) and mouse antiparvalbumin (dilution 1:5000; Swant, Bellinzona, Switserland), for 2 days. After washing, sections were incubated with donkey antirabbit alexa 594 (1:200, Invitrogen, Breda, the Netherlands) and donkey anti-mouse alexa 488 (1:200, Invitrogen, Breda, the Netherlands). Sections were then washed, mounted on gelatincoated glasses and coverslipped with $80 \%$ glycerol in TBS. Stained sections were photographed (4x magnification) using a camera (Olympus DP70) mounted on a bright-field microscope (Olympus AX70). Cells immunopositive for c-Fos were counted automatically (cell P program, Olympus) as described previously (Moers-Hornikx, et al., 2009). The mean number of 
cells was corrected for surface area and expressed as cells/mm2. Fluorescent parvalbumin/c-Fos double-labeled cells were visualized using a Disk Spinning Unit microscope (Olympus DSU BX51WI, Pennsylvania, USA). Typically, parvalbumin-positive cells had a green-stained cytoplasm with adjoining axon whereas c-Fos-positive cells had a deep red nuclear staining. Co-localization of these fluorescent signals in a single cell was evaluated using confocal imaging.

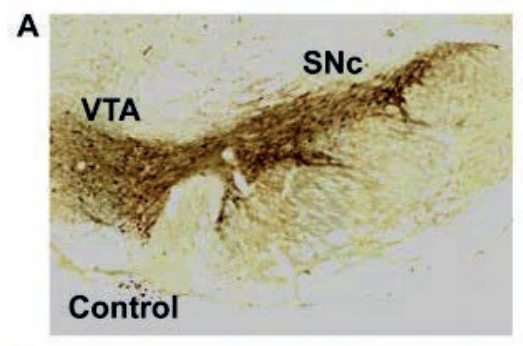

B

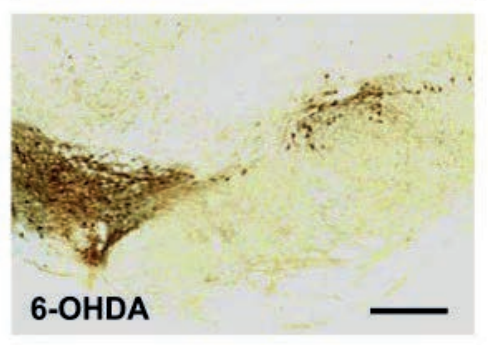

TH quantification

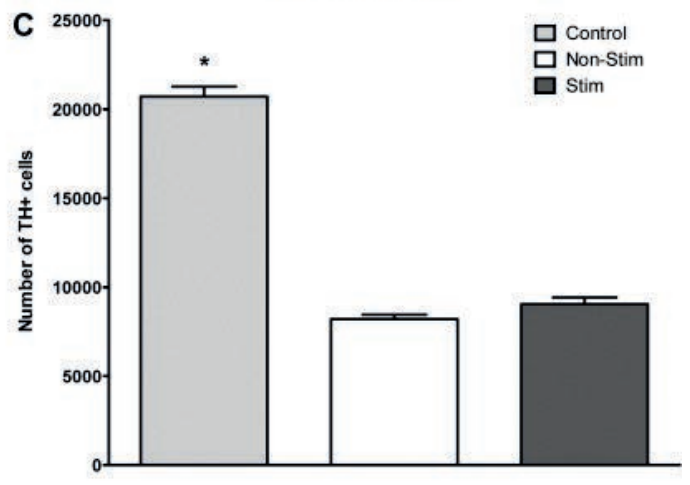

D

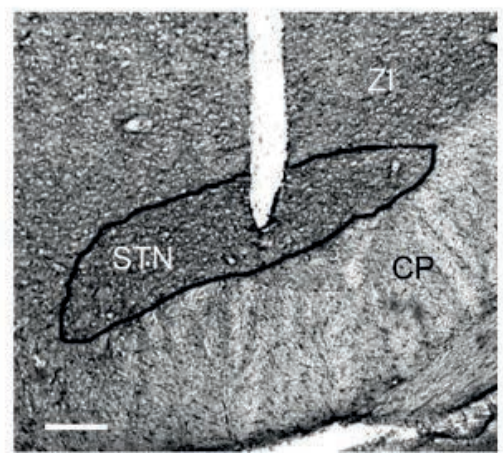

Fig. 1. Effect of 6-OHDA lesions on TH expression in the SNc. TH immunocytochemistry of a control rat (A) and a 6-OHDA treated rat (B) (SNc=substantia nigra compacta; VTA = ventral tegmental area; scale bar $=500 \mu \mathrm{m})$. Grouped data for TH-positive neurons in the SNc presented in means \pm s.e.m $(C),{ }^{*} p<0.05$ control versus non-stim and stim groups. Illustrative coronal section that shows the histological verification of the electrode location in the STN (CP = cerebral peduncle; STN = subthalamic nucleus; ZI = zona incerta; bar $=150 \mu \mathrm{m})(\mathrm{D})$.

\section{Cytochrome Coxidase histochemistry}

A series of brain sections were processed for cytochrome C oxidase (COX) histochemistry. COX activity was used as a marker of metabolic activity in the DRN. Tissue sections were mounted on gelatin-coated slides and air-dried overnight prior to incubation in a nickel enhanced 0.1 MHEPES solution (1\% nickel chloride, $\mathrm{pH}$ 7.4) containing $0.0224 \%$ cytochrome C oxidase (equine heart, Sigma Aldrich, the Netherlands), 0.115\% DAB and 
$4.5 \%$ sucrose. Sections were incubated at $37{ }^{\circ} \mathrm{C}$ under constant shaking in the dark for 6 $\mathrm{h}$ before being transferred to a neutral buffered formaldehyde medium for 10 min to stop the reaction. This was followed by ethanol dehydration, xylene treatment and DEPEX fixation. COX activity was analyzed using a slightly modified method described previously (Kaya, et al., 2008). COX activity was quantified by measurement of inversed optical density (ImageJ software version $1.41^{\circ}$; U.S. National Institutes of Health, Bethesda, USA) in digital photographs taken using a light microscope (Olympus AX70) and connected to a digital camera (Olympus U-CMAD-2; analySIS imaging system, Münster, Germany). The inversed optical density, which is inversely related to COX activity (i.e. value of zero is a dark staining and reflects high metabolic activity), was obtained from 3 DRN images per rat (AP -7.6 mm, -7.8 mm and $-8.0 \mathrm{~mm}$ ) and corrected for measured surface area $\left(\mathrm{mm}^{2}\right)$.

\section{Statistical analysis}

Data are presented as mean \pm S.E.M. values. Histological and behavioral data were analyzed statistically using a one-way ANOVA with an LSD post-hoc multiple comparisons test. Because STN stimulation was performed bilaterally, data from bilateral structures were pooled. All statistical analyses were performed with SPSS 15.0 version for Windows. P values lower than 0.05 were considered statistically significant.

\section{Results}

Validation of dopamine lesions and electrode implantations

Rats receiving bilateral intra-striatal injections of 6-OHDA demonstrated a significant reduction in TH-positive cells in the SNc compared to sham-controls $(p<0.01$, Fig. $1 \mathrm{~A}-\mathrm{C}$ ). Stimulating electrodes were positioned bilaterally and symmetrically (interelectrode variation $<0.1 \mathrm{~mm}$ ) in the STN in all rats except two, for which electrodes were located in the zona incerta. The latter rats were excluded from the analysis. No signs of significant histological damage due to implantation or chronic stimulation were observed (Fig. 1D).

\section{Effect of HFS of the STN in FST and social interaction test}

In the FST, bilateral HFS of the STN caused a significant increase in immobility time compared to non-stimulated (stimulation off) controls, indicating increased behavioral despair (Fig. 2A). In the social interaction task, HFS of the STN evoked significantly more sniffing behavior in comparison to non-stimulated rats $(p<0.05)$. However, crawling and following behavior remained unchanged (Fig. 2B). The increase in social interaction is in line with a previous report showing increased social interaction in 5-HT-depleted rats (Collins, et al., 1979). 


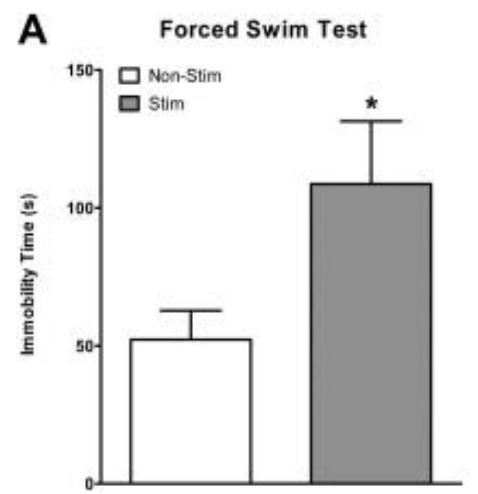

\section{B}

Social Interaction Test

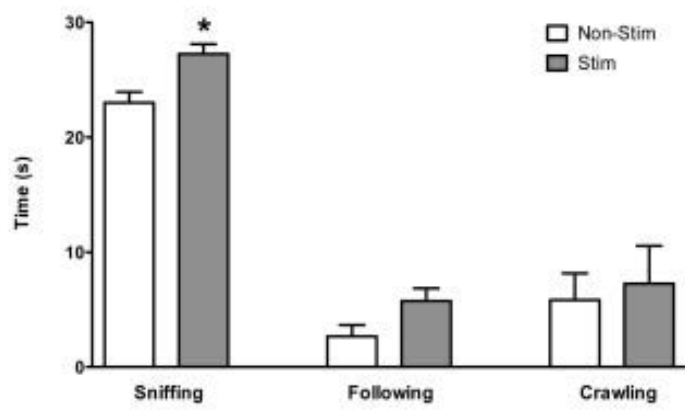

Fig. 2. Effects of HFS of the STN on immobility time in the forced swim test (A) and sniffing behavior in the social interaction test (B). Mean time \pm s.e.m values are shown. $\left({ }^{*} p<0.05\right.$ stim versus non-stim rats).

Effect of HFS of the STN on c-Fos immunoreactivity in the DRN

Bilateral HFS of the STN increased the number of c-Fos positive neurons in the DRN compared to non-stimulated controls (Fig. 3A-D). This effect was apparent in both median and lateral subdivisions of the DRN. Thus, c-Fos counts in the median subdivisions were greater in stimulated compared to non-stimulated rats (stimulated $7.13 \pm 0.41$ vs control $2.90 \pm 0.75$ cells $/ \mathrm{mm} 2$ and non-stimulated $3.39 \pm 2.30$ cells $/ \mathrm{mm} 2 ; p<0.01$ ), and this was also the case for the lateral subdivisions (stimulated vs control; respectively12.99 \pm 0.92 and $5.92 \pm 1.66$ cells $/ \mathrm{mm} 2 ; \mathrm{p}<0.05$ ). In STN stimulated rats the number of c-Fos positive cells in the lateral subdivisions was higher compared to the median subdivisions (lateral wings vs central DRN; respectively $12.99 \pm 0.92$ vs $7.13 \pm 0.41$ cells/mm2; $P<0.01$ ). To characterize the identity of the c-Fos-positive DRN neurons, sections were assessed for immunofluorescent double-labeling with parvalbumin, which marks GABA neurons. Parvalbumin positive cells were predominantly present in the lateral aspects of the DRN. HFS of the STN evoked immunofluorescent c-Fos positive cells throughout the DRN, as described above. Qualitative assessment showed parvalbumin/c-Fos co-localized cells situated within the lateral aspects (Fig. 4). No doublelabeled cells have been observed in the median parts of the DRN. 
A

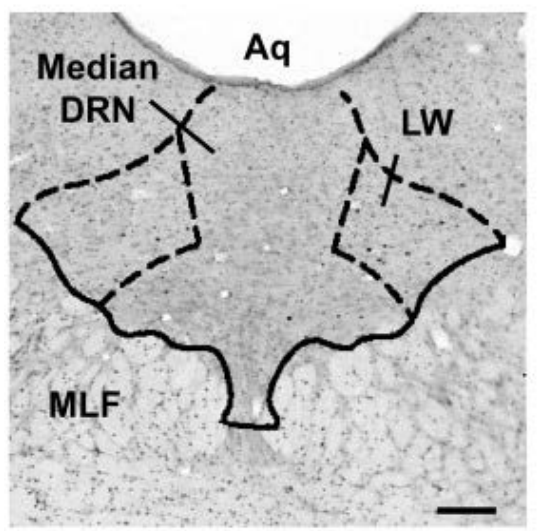

C

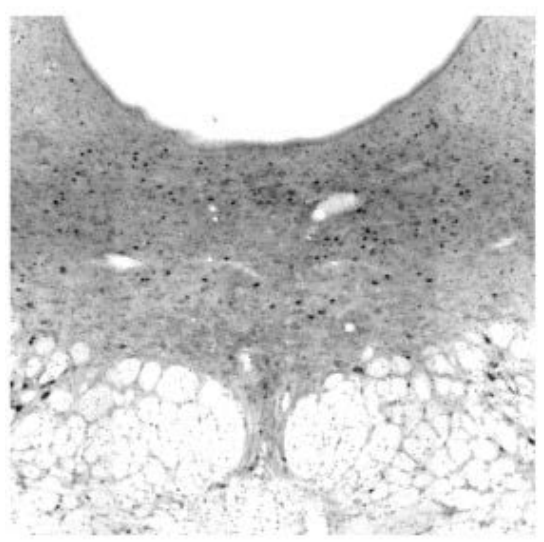

B

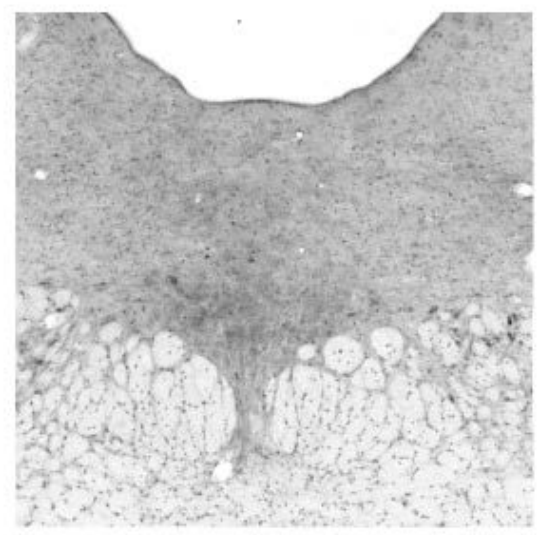

D DRN c-fos expression

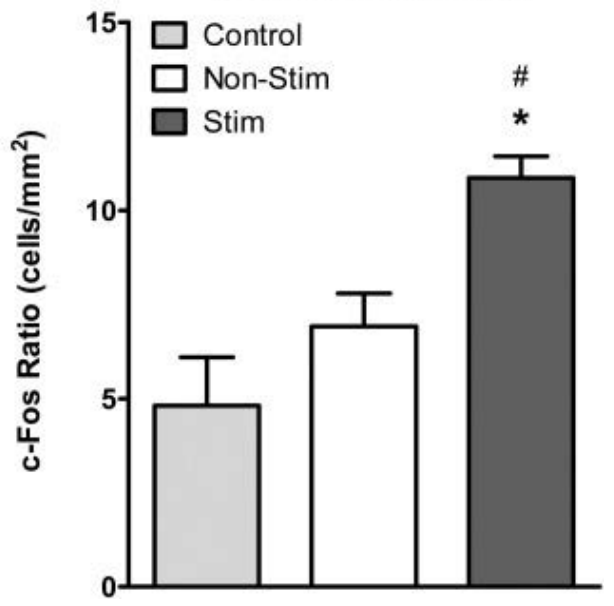

Fig. 3. Effect of HFS of the STN on c-fos expression in the DRN. Representative pictures taken from a nonstimulated control (A), non-stimulated 6-OHDA (B) and stimulated 6-OHDA rat (C). Cumulative data of c-fos neurons in the DRN (D), $* p<0.01$; stim vs control; $\# p<0.05$ stim vs non-stim. Aq: aqueduct, MLF: medial longitudinal fasciculus, LW: DRN lateral wing (bar $=200 \mu \mathrm{m})$.

\section{Effect of HFS of the STN on COX activity in the DRN}

Experiments examined the effect of HFS of the STN on metabolic activity in the DRN, as assessed by COX activity. It was found that compared to non-stimulated controls, HFS of the STN significantly increased the inversed optical density measurements of COX in the DRN $(P<0.05)$, indicative of an overall lower level of metabolic activity in this region (Fig. 5A-D). Interestingly, COX activity in the DRN of dopamine-lesioned rats was significantly higher than non-lesioned rats $(P<0.05$; i.e. decreased inversed optical density ratio). This is in line with our previous findings (Kaya, et al., 2008). 


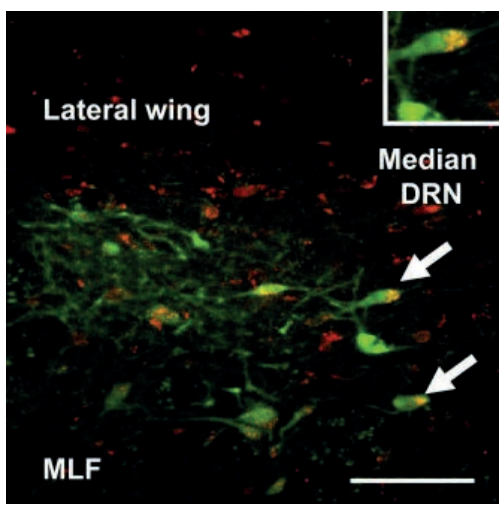

Fig. 4. Effect of HFS of the STN on GABA neurons in the DRN lateral wings. A representative high power confocal fluorescent image of parvalbumin (green) and c-fos (red) double-labeled cells, which are located in the DRN lateral wing and indicated by the arrows. MLF: medial longitudinal fasciculus (bar $=200 \mu \mathrm{m})$.

A

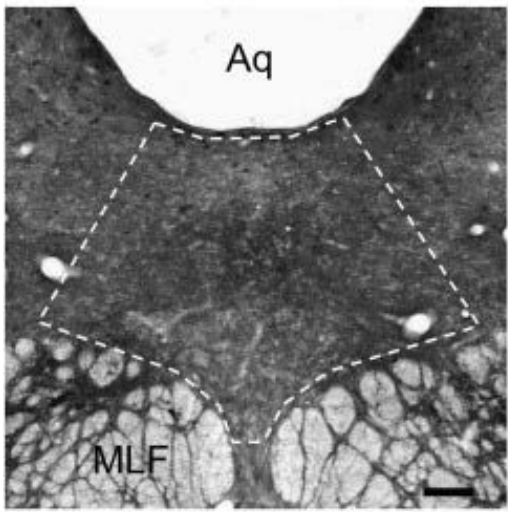

C

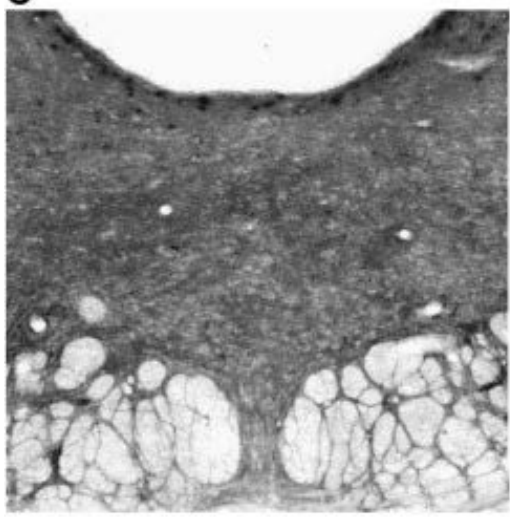

B

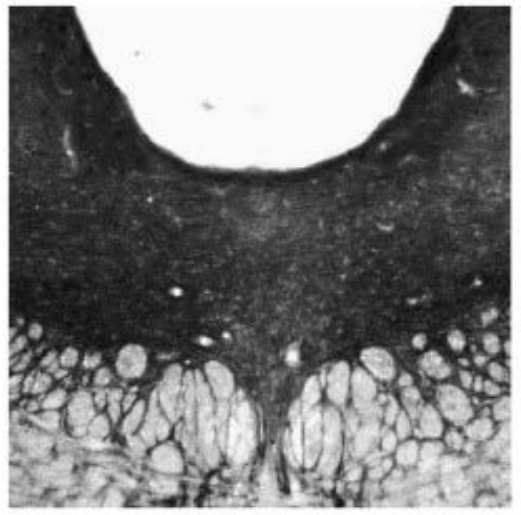

DRN Cytochrome C Oxidase

D

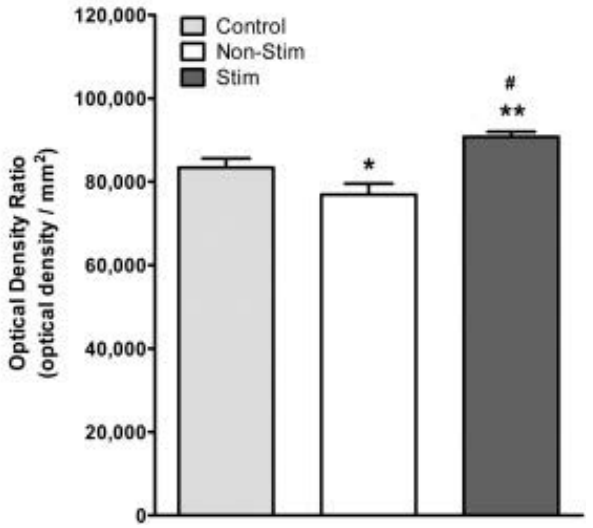

Fig. 5. Effect of HFS of the STN on COX activity in the DRN. Representative pictures of the DRN region of a non-stimulated control rat (A), non-stimulated 6-OHDA rat (B) and stimulated 6-OHDA rat (C). The white dashed line represents the delineation of the DRN used to measure optical density ratio (A). Cumulative data of DRN inverse optical density ratio (mean \pm s.e.m.) (D). ${ }^{*} p<0.05$ Inversed optical density ratio non-stim vs control. ${ }^{* *} p<0.05$ stim vs control and $\# p<0.001$ stim vs non-stim. Aq: aqueduct, MLF: medial longitudinal fasciculus (bar $=200 \mu \mathrm{m})$. 
Effect of HFS of the STN on c-Fos immunoreactivity in MPFC, LHb and SN

Experiments investigated the effect of HFS of the STN on c-Fos immunoreactivity in specific regions with direct neural projections to the DRN. In STN stimulated rats, the number of c-Fos positive neurons in the medial part of the LHb was significantly greater than non-stimulated controls $(P<0.05$ ) (Fig. 6A-D). In addition, the number of c-Fos positive cells in the prelimbic cortex was greater in STN stimulated compared to nonstimulated rats, whereas c-Fos counts in the infralimbic cortex was not different between groups (Fig. 6E-H). Finally, the number of c-Fos positive neurons in the SNr and SNc tended to be greater in STN stimulated versus non-stimulated control rats but this effect was not statistically significant ( $P>0.05$, Fig. 6I-J).

A

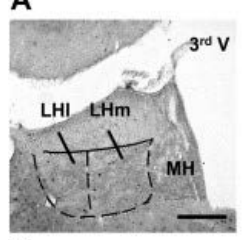

C

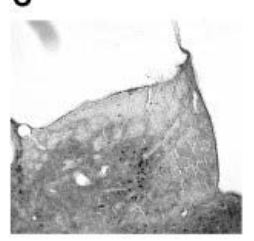

B

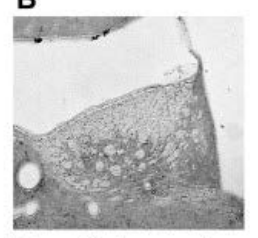

D L L c.Fos expression

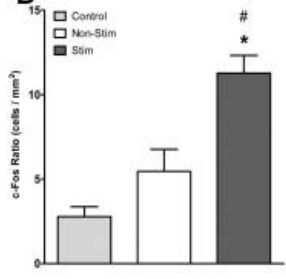

I

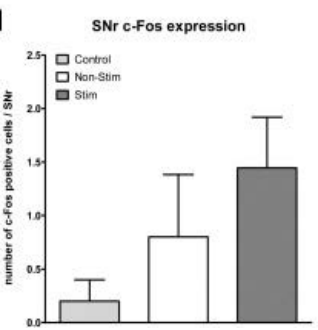

E
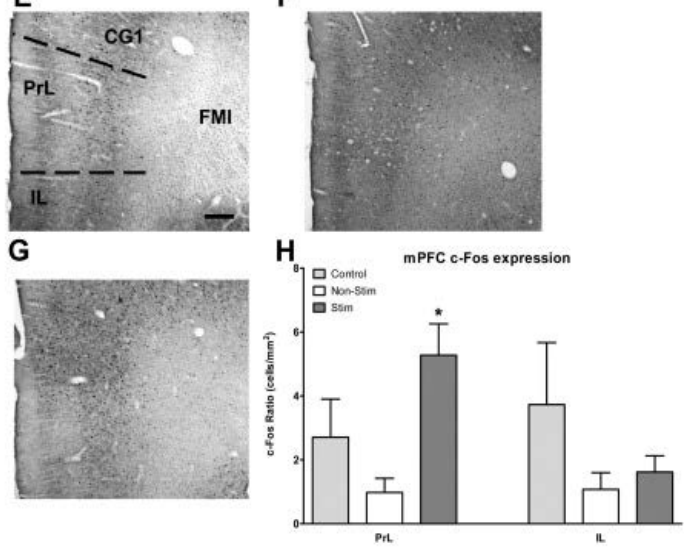

$\mathbf{F}$

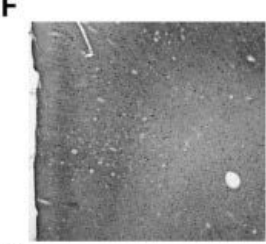

$\mathbf{J}$

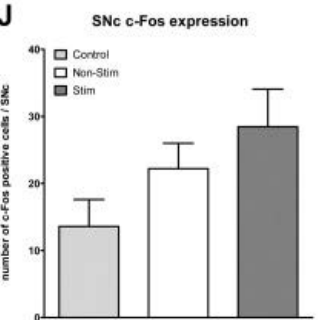

Fig. 6. Effect of HFS of the STN on DRN afferent nuclei. Expression of c-fos in the medial aspect of the LHb and mPFC of non-stimulated control (A, E), non-stimulated 6-OHDA (B, F) and stimulated 6-OHDA rats (C, G). Cumulative data in means \pm s.e.m for the LHb ( ${ }^{*} p<0.001$ stim vs control; $\# p<0.01$ stim vs non-stim) (D) and mPFC ( ${ }^{*} p<0.05$ stim vs non-stim) (H). No change in c-fos expression in the SNr (I) and the SNc (J) after STN HFS. 3rdV: Third ventricle, CG1: cingulate cortex 1, FMI: forceps minor of the corpus callosum, IL: infralimbic cortex, LHI: laterolateral habenula, LHm: mediolateral habenula, MH: medial habenula, PrL: prelimbic cortex $($ bar $=200 \mu \mathrm{m})$. 


\section{Discussion}

Although HFS of the STN improves motor disability in patients with advanced PD, psychiatric side-effects overshadow these therapeutic benefits in some patients (Temel, et al., 2006, Troster, et al., 2003). Indeed, clinical depression has recently been recognized as a major risk factor for post-operative suicide associated with HFS of the STN (Soulas, et al., 2008, Voon, et al., 2008). Depression is linked to low 5-HT, and ourselves and others have recently reported that in rodent PD models, HFS of the STN inhibits the firing of 5-HT neurons in the DRN and decreases extracellular5-HT in forebrain regions (Hartung, et al., 2011, Navailles, et al., 2010, Tan, et al., 2010, Temel, et al., 2007). The neural circuitry, which mediates the inhibitory effect of HFS of the STN on the 5-HT system, is unknown. The current experiments demonstrated that HFS of the STN increased c-Fos immunoreactivity in the DRN and in specific DRN input regions. Moreover, findings indicated that this increase in c-Fos immunoreactivity in the DRN colocalized in neurons expressing the GABAergic marker, parvalbumin.

Initial experiments demonstrated that rats with bilateral intrastriatal injections of the dopamine neurotoxin 6-OHDA had a marked loss of TH immunoreactivity in the SNc, indicative of an extensive loss of ascending dopamine neurons that marks PD. In these animals, HFS of the STN at clinically relevant stimulation parameters, both increased immobility in the FST and increased sniffing in a social interaction test. Both effects are consistent with decreased 5-HT transmission (Collins, et al., 1979, Cryan, et al., 2002, Higgins, et al., 1988, Higgins, et al., 1992, Hogg, et al., 1994), and previously we found that the increase in immobility induced by HFS of the STN was prevented by SSRI treatment (Temel, et al., 2007).

At the same stimulation parameters used in the behavioral experiments, HFS of the STN reduced the activity of COX in the DRN, suggesting an overall decrease in metabolic activity in this region over a longer period of time. This reduction came on top of elevated baseline COX activity in non-stimulated 6-OHDA animals that was apparent when compared to naive controls. The finding of elevated COX activity in the DRN of 6-OHDA lesioned rats confirms our earlier study (Kaya, et al., 2008), which also reported increased firing of DRN 5-HT neurons in such animals as also observed by others (Wang, et al., 2009, Zhang, et al., 2007).

Since 5-HT neurons comprise the large majority (around 50\%) of neurons in the DRN (Steinbusch, 1981), it seems entirely plausible that the decrease in metabolic activity measured by COX activity evoked by HFS of the STN, reflects the decrease in 5-HT cell firing detected in electrophysiological studies (Hartung, et al., 2011, Temel, et al., 2007). To better understand the chemical identity of DRN neurons modulated by HFS of the STN immunoreactivity of the immediate early gene c-Fos was measured in the DRN. It was found that HFS of the STN increased c-Fos immunoreactivity in DRN neurons, and 
this effect was apparent across specific DRN subdivisions. Distinct forebrain projections arise from DRN subdivisions (Commons, et al., 2003, Jacobs, et al., 1978, Molliver, 1987, O'Hearn and Molliver, 1984) and these may regulate specific behavioral responses (Roche, et al., 2003). Interestingly, c-Fos positive neurons were located mostly in the lateral wings of the DRN, which are abundant in GABA neurons (Allers and Sharp, 2003). This result is in line with previous data showing swim stress to induce c-Fos expression in the lateral DRN (Roche, et al., 2003). Moreover, double-labeling experiments revealed that these c-Fos-positive neurons co-localized the GABA neuron marker parvalbumin, which is present in a subset of putative GABA neurons in the DRN (Charara and Parent, 1998). It should also be noted that some c-Fos-positive neurons was observed in the medial DRN that were not parvalbumin-positive. This finding is consistent with our recent observation that a subpopulation of putative 5 -HT neurons (24\%) was excited by HFS of the STN (Hartung, et al., 2011. However, microdialysis data suggest that the overall effect of HFS of the STN on the 5-HT system is inhibitory \{Navailles, 2010 \#235, Tan, et al., 2010). Moreover, it should be pointed out that the medial DRN also contains many non-5-HT containing neurons (Charara and Parent, 1998, Fu, et al., 2010). Taken together, these data support the idea that the main effect of HFS of the STN in the DRN is to activate local GABA neurons, which synapse with and inhibit nearby 5-HT neurons.

Since there is no evidence of a direct projection from the STN to the DRN, the DRN GABA neurons are likely activated by afferent inputs. Interestingly, HFS of the STN increased c-Fos immunoreactivity in the LHb. Previous neuroanatomical and electrophysiological data suggest that there is an excitatory glutamate projection from the LHb to the DRN, which selectively activates GABA neurons to inhibit nearby 5-HT neurons (Aghajanian and Wang, 1977, Sharp, et al., 2007, Varga, et al., 2003, Wang and Aghajanian, 1977). Although the present study did not prove that the LHb neurons activated by HFS of the STN, projected to the DRN, the c-Fos positive neurons were located in the medial aspect of the LHb that is the primary source of DRN afferents (Aghajanian and Wang, 1977, Peyron, et al., 1998). Therefore, excitatory projections from the LHb to the DRN GABA neurons are a candidate neural substrate for the inhibition of DRN 5-HT neurons by HFS of the STN. The STN does not, however, directly project to the LHb but indirectly through the ventral pallidum and entopeduncular nucleus (Groenewegen and Berendse, 1990, Groenewegen, et al., 1993, Nagy, et al., 1978).

HFS of the STN also increased c-Fos immunoreactivity in the prelimbic cortex of the mPFC, another source of input to the DRN (Hajos, et al., 1998, Jankowski and Sesack, 2004, Peyron, et al., 1998, Sesack, et al., 1989, Vertes, 2006). Indeed, electrical stimulation of the MPFC, including the prelimbic area, inhibits 5-HT cell firing in the DRN via activation of DRN GABAergic neurons (Hajos, et al., 1998, Varga, et al., 2003, Varga, et al., 2001). The STN is known to project to the mPFC directly via a subthalamic-cortical projection (Degos, et al., 2008) but also indirectly through basal ganglia-thalamocortical 
connections (Temel, et al., 2005). A recent study using optogenetic techniques suggested that subthalamic-cortical connections contribute to the effects of HFS of STN (Gradinaru, et al., 2009). Thus, another candidate substrate for the inhibition of 5-HT neurons is the excitatory input from the prelimbic cortex to the DRN GABA neurons.

Another candidate pathway for the inhibition of 5-HT neurons by HFS of the STN is the SN. The SN projects to the DRN and receives powerful inputs from the STN (Kalen, et al., 1988, Kirouac, et al., 2004, Kirouac and Pittman, 2000). Part of the evidence for a role for the SN are case reports that HFS of this brain area induces acute depressive symptoms (Bejjani, et al., 1999, Blomstedt, et al., 2008). In the present study, HFS of the STN caused a tendency for an increase in the number of c-Fos immunoreactive cells in the SNr and SNc, but this effect was not statistically significant. Although STN-SN-DRN connectivity does not show up as a potential substrate for the inhibition of 5-HT cell firing, it cannot be completely ruled out.

In summary, the current data show that in an animal model of PD, HFS of the STN, at clinically relevant stimulation parameters which evoked changes in learned helplessness and social interaction increased c-Fos immunoreactivity in DRN GABA neurons and decreased metabolic activity in this region. HFS of the STN also increased c-Fos immunoreactivity in neurons in the LHb and prelimbic cortex which are DRN input regions. These data are consistent with the view that HFS of the STN activates DRN GABA neurons to inhibit 5-HT neuronal activity and trigger mood changes, and that DRN afferents from the LHb and MPFC may be involved.

\section{Acknowledgements}

Funding for this project was provided by the Netherlands Organization for Scientific Research (NWO) (Mosaic grant no 017.003.015, and Veni grant no: 016.096.032), the Dutch Brain Foundation (Hersenstichting Nederland grant nos: 2008-32 and 2009-26), and Parkinson's UK. The funding sources had no involvement in study design, data collection, analysis and interpretation, writing and submission. We thank Dr. Ramazan Kozan and Dorien Merken for their technical assistance. 


\section{References}

1. Aghajanian, G. K., and Wang, R. Y., 1977. Habenular and other midbrain raphe afferents demonstrated by a modified retrograde tracing technique. Brain Res 122, 229-242.

2. Allers, K. A., and Sharp, T., 2003. Neurochemical and anatomical identification of fast- and slow-firing neurones in the rat dorsal raphe nucleus using juxtacellular labelling methods in vivo. Neuroscience 122, 193-204.

3. Bejjani, B. P., Damier, P., Arnulf, I., Thivard, L., Bonnet, A. M., Dormont, D., Cornu, P., Pidoux, B., Samson, Y., and Agid, Y., 1999. Transient acute depression induced by high-frequency deep-brain stimulation. $N$ Engl J Med 340, 1476-1480.

4. Berney, A., Vingerhoets, F., Perrin, A., Guex, P., Villemure, J. G., Burkhard, P. R., Benkelfat, C., and Ghika, J., 2002. Effect on mood of subthalamic DBS for Parkinson's disease: a consecutive series of 24 patients. Neurology 59, 1427-1429.

5. Blomstedt, P., Hariz, M. I., Lees, A., Silberstein, P., Limousin, P., Yelnik, J., and Agid, Y., 2008. Acute severe depression induced by intraoperative stimulation of the substantia nigra: a case report. Parkinsonism Relat Disord 14, 253-256.

6. Celio, M. R., 1990. Calbindin D-28k and parvalbumin in the rat nervous system. Neuroscience 35, 375475.

7. Charara, A., and Parent, A., 1998. Chemoarchitecture of the primate dorsal raphe nucleus. Journal of chemical neuroanatomy 15, 111-127.

8. Collins, G. G., File, S. E., Hyde, J. R., and Macleod, N. K., 1979. The effects of 5,7-dihydroxytryptamine lesions of the median and of the dorsal raphe nuclei on social interaction in the rat [proceedings]. British journal of pharmacology 66, 114P-115P.

9. Commons, K. G., Connolley, K. R., and Valentino, R. J., 2003. A neurochemically distinct dorsal raphelimbic circuit with a potential role in affective disorders. Neuropsychopharmacology : official publication of the American College of Neuropsychopharmacology 28, 206-215.

10. Cryan, J. F., Markou, A., and Lucki, I., 2002. Assessing antidepressant activity in rodents: recent developments and future needs. Trends in pharmacological sciences 23, 238-245.

11. Degos, B., Deniau, J. M., Le Cam, J., Mailly, P., and Maurice, N., 2008. Evidence for a direct subthalamocortical loop circuit in the rat. Eur J Neurosci 27, 2599-2610.

12. Deuschl, G., Schade-Brittinger, C., Krack, P., Volkmann, J., Schafer, H., Botzel, K., Daniels, C., Deutschlander, A., Dillmann, U., Eisner, W., Gruber, D., Hamel, W., Herzog, J., Hilker, R., Klebe, S., Kloss, M., Koy, J., Krause, M., Kupsch, A., Lorenz, D., Lorenzl, S., Mehdorn, H. M., Moringlane, J. R., Oertel, W., Pinsker, M. O., Reichmann, H., Reuss, A., Schneider, G. H., Schnitzler, A., Steude, U., Sturm, V., Timmermann, L., Tronnier, V., Trottenberg, T., Wojtecki, L., Wolf, E., Poewe, W., and Voges, J., 2006. A randomized trial of deep-brain stimulation for Parkinson's disease. N Engl J Med 355, 896-908.

13. File, S. E., and Hyde, J. R., 1978. Can social interaction be used to measure anxiety? British journal of pharmacology 62, 19-24.

14. Fu, W., Le Maitre, E., Fabre, V., Bernard, J. F., David Xu, Z. Q., and Hokfelt, T., 2010. Chemical neuroanatomy of the dorsal raphe nucleus and adjacent structures of the mouse brain. J Comp Neurol 518, 3464-3494.

15. Gradinaru, V., Mogri, M., Thompson, K. R., Henderson, J. M., and Deisseroth, K., 2009. Optical Deconstruction of Parkinsonian Neural Circuitry. Science.

16. Groenewegen, H. J., and Berendse, H. W., 1990. Connections of the subthalamic nucleus with ventral striatopallidal parts of the basal ganglia in the rat. J Comp Neurol 294, 607-622.

17. Groenewegen, H. J., Berendse, H. W., and Haber, S. N., 1993. Organization of the output of the ventral striatopallidal system in the rat: ventral pallidal efferents. Neuroscience 57, 113-142.

18. Hajos, M., Richards, C. D., Szekely, A. D., and Sharp, T., 1998. An electrophysiological and neuroanatomical study of the medial prefrontal cortical projection to the midbrain raphe nuclei in the rat. Neuroscience 87, 95-108. 
19. Hartung, H., Tan, S. K., Steinbusch, H. M., Temel, Y., and Sharp, T., 2011. High-frequency stimulation of the subthalamic nucleus inhibits the firing of juxtacellular labelled 5-HT-containing neurones. Neuroscience 186, 135-145.

20. Higgins, G. A., Bradbury, A. J., Jones, B. J., and Oakley, N. R., 1988. Behavioural and biochemical consequences following activation of 5HT1-like and GABA receptors in the dorsal raphe nucleus of the rat. Neuropharmacology 27, 993-1001.

21. Higgins, G. A., Jones, B. J., and Oakley, N. R., 1992. Effect of 5-HT1A receptor agonists in two models of anxiety after dorsal raphe injection. Psychopharmacology (Berl) 106, 261-267.

22. Hogg, S., Andrews, N., and File, S. E., 1994. Contrasting behavioural effects of 8-OH DPAT in the dorsal raphe nucleus and ventral hippocampus. Neuropharmacology 33, 343-348.

23. Houeto, J. L., Mesnage, V., Mallet, L., Pillon, B., Gargiulo, M., du Moncel, S. T., Bonnet, A. M., Pidoux, B., Dormont, D., Cornu, P., and Agid, Y., 2002. Behavioural disorders, Parkinson's disease and subthalamic stimulation. J Neurol Neurosurg Psychiatry 72, 701-707.

24. Jacobs, B. L., Foote, S. L., and Bloom, F. E., 1978. Differential projections of neurons within the dorsal raphe nucleus of the rat: a horseradish peroxidase (HRP) study. Brain Res 147, 149-153.

25. Jankowski, M. P., and Sesack, S. R., 2004. Prefrontal cortical projections to the rat dorsal raphe nucleus: ultrastructural features and associations with serotonin and gamma-aminobutyric acid neurons. J Comp Neurol 468, 518-529.

26. Kalen, P., Skagerberg, G., and Lindvall, O., 1988. Projections from the ventral tegmental area and mesencephalic raphe to the dorsal raphe nucleus in the rat. Evidence for a minor dopaminergic component. Experimental brain research. Experimentelle Hirnforschung. Experimentation cerebrale 73, 69-77.

27. Kask, A., Nguyen, H. P., Pabst, R., and von Horsten, S., 2001. Factors influencing behavior of grouphoused male rats in the social interaction test: focus on cohort removal. Physiology \& behavior 74, $277-$ 282.

28. Kaya, A. H., Vlamings, R., Tan, S., Lim, L. W., Magill, P. J., Steinbusch, H. W., Visser-Vandewalle, V., Sharp, T., and Temel, Y., 2008. Increased electrical and metabolic activity in the dorsal raphe nucleus of Parkinsonian rats. Brain Res 1221, 93-97.

29. Kirouac, G. J., Li, S., and Mabrouk, G., 2004. GABAergic projection from the ventral tegmental area and substantia nigra to the periaqueductal gray region and the dorsal raphe nucleus. J Comp Neurol 469, 170-184.

30. Kirouac, G. J., and Pittman, Q. J., 2000. A projection from the ventral tegmental area to the periaqueductal gray involved in cardiovascular regulation. American journal of physiology. Regulatory, integrative and comparative physiology 278, R1643-1650.

31. Liu, R., Jolas, T., and Aghajanian, G., 2000. Serotonin 5-HT(2) receptors activate local GABA inhibitory inputs to serotonergic neurons of the dorsal raphe nucleus. Brain Res 873, 34-45.

32. Mann, J. J., 2003. Neurobiology of suicidal behaviour. Nature reviews. Neuroscience 4, 819-828.

33. Moers-Hornikx, V. M., Sesia, T., Basar, K., Lim, L. W., Hoogland, G., Steinbusch, H. W., Gavilanes, D. A., Temel, Y., and Vles, J. S., 2009. Cerebellar nuclei are involved in impulsive behaviour. Behav Brain Res 203, 256-263.

34. Molliver, M. E., 1987. Serotonergic neuronal systems: what their anatomic organization tells us about function. Journal of clinical psychopharmacology 7, 3S-23S.

35. Nagy, J. I., Carter, D. A., Lehmann, J., and Fibiger, H. C., 1978. Evidence for a GABA-containing projection from the entopeduncular nucleus to the lateral habenula in the rat. Brain Res 145, 360-364.

36. Navailles, S., Benazzouz, A., Bioulac, B., Gross, C., and De Deurwaerdere, P., 2010. High-frequency stimulation of the subthalamic nucleus and L-3,4-dihydroxyphenylalanine inhibit in vivo serotonin release in the prefrontal cortex and hippocampus in a rat model of Parkinson's disease. J Neurosci 30, 2356-2364.

37. O'Hearn, E., and Molliver, M. E., 1984. Organization of raphe-cortical projections in rat: a quantitative retrograde study. Brain Res Bull 13, 709-726.

38. Paxinos, G., and Watson, C., 1998. The Rat Brain in Stereotaxic Coordinates. Academic Press, New York. 
39. Peyron, C., Petit, J. M., Rampon, C., Jouvet, M., and Luppi, P. H., 1998. Forebrain afferents to the rat dorsal raphe nucleus demonstrated by retrograde and anterograde tracing methods. Neuroscience 82 , 443-468.

40. Roche, M., Commons, K. G., Peoples, A., and Valentino, R. J., 2003. Circuitry underlying regulation of the serotonergic system by swim stress. The Journal of neuroscience : the official journal of the Society for Neuroscience 23, 970-977.

41. Schmitz, C., and Hof, P. R., 2005. Design-based stereology in neuroscience. Neuroscience 130, 813-831.

42. Schrag, A., Jahanshahi, M., and Quinn, N., 2000. What contributes to quality of life in patients with Parkinson's disease? J Neurol Neurosurg Psychiatry 69, 308-312.

43. Sesack, S. R., Deutch, A. Y., Roth, R. H., and Bunney, B. S., 1989. Topographical organization of the efferent projections of the medial prefrontal cortex in the rat: an anterograde tract-tracing study with Phaseolus vulgaris leucoagglutinin. J Comp Neurol 290, 213-242.

44. Sharp, T., Boothman, L., Raley, J., and Queree, P., 2007. Important messages in the 'post': recent discoveries in 5-HT neurone feedback control. Trends in pharmacological sciences 28, 629-636.

45. Smith, K. A., Fairburn, C. G., and Cowen, P. J., 1997. Relapse of depression after rapid depletion of tryptophan. Lancet 349, 915-919.

46. Soulas, T., Gurruchaga, J. M., Palfi, S., Cesaro, P., Nguyen, J. P., and Fenelon, G., 2008. Attempted and completed suicides after subthalamic nucleus stimulation for Parkinson's disease. J Neurol Neurosurg Psychiatry 79, 952-954.

47. Steinbusch, H. W., 1981. Distribution of serotonin-immunoreactivity in the central nervous system of the rat-cell bodies and terminals. Neuroscience 6, 557-618.

48. Tan, S., Vlamings, R., Lim, L., Sesia, T., Janssen, M. L., Steinbusch, H. W., Visser-Vandewalle, V., and Temel, Y., 2010. Experimental deep brain stimulation in animal models. Neurosurgery 67, 1073-1079; discussion1080.

49. Tan, S. K. H., Hartung, H., Temel, Y., and Sharp, T., High frequency stimulation of the subthalamic nucleus inhibts the firing of juxtacellular labelled 5-HT-containing neurons and decreases 5-HT release in vivo., Society for Neuroscience, San Diego, 2010.

50. Temel, Y., Boothman, L. J., Blokland, A., Magill, P. J., Steinbusch, H. W., Visser-Vandewalle, V., and Sharp, T., 2007. Inhibition of 5-HT neuron activity and induction of depressive-like behavior by high-frequency stimulation of the subthalamic nucleus. Proc Natl Acad Sci U S A 104, 17087-17092.

51. Temel, Y., Kessels, A., Tan, S., Topdag, A., Boon, P., and Visser-Vandewalle, V., 2006. Behavioural changes after bilateral subthalamic stimulation in advanced Parkinson disease: a systematic review. Parkinsonism Relat Disord 12, 265-272.

52. Temel, Y., Visser-Vandewalle, V., Aendekerk, B., Rutten, B., Tan, S., Scholtissen, B., Schmitz, C., Blokland, A., and Steinbusch, H. W., 2005. Acute and separate modulation of motor and cognitive performance in parkinsonian rats by bilateral stimulation of the subthalamic nucleus. Exp Neurol 193, 43-52.

53. Temel, Y., Visser-Vandewalle, V., Kaplan, S., Kozan, R., Daemen, M. A., Blokland, A., Schmitz, C., and Steinbusch, H. W., 2006. Protection of nigral cell death by bilateral subthalamic nucleus stimulation. Brain Res 1120, 100-105.

54. Temel, Y., Visser-Vandewalle, V., van der Wolf, M., Spincemaille, G. H., Desbonnet, L., Hoogland, G., and Steinbusch, H. W., 2004. Monopolar versus bipolar high frequency stimulation in the rat subthalamic nucleus: differences in histological damage. Neurosci Lett 367, 92-96.

55. Tonissaar, M., Herm, L., Eller, M., Koiv, K., Rinken, A., and Harro, J., 2008. Rats with high or low sociability are differently affected by chronic variable stress. Neuroscience 152, 867-876.

56. Troster, A. I., Fields, J. A., Wilkinson, S., Pahwa, R., Koller, W. C., and Lyons, K. E., 2003. Effect of motor improvement on quality of life following subthalamic stimulation is mediated by changes in depressive symptomatology. Stereotact Funct Neurosurg 80, 43-47.

57. Van den Hove, D. L., Blanco, C. E., Aendekerk, B., Desbonnet, L., Bruschettini, M., Steinbusch, H. P., Prickaerts, J., and Steinbusch, H. W., 2005. Prenatal restraint stress and long-term affective consequences. Developmental neuroscience 27, 313-320. 
58. Varga, V., Kocsis, B., and Sharp, T., 2003. Electrophysiological evidence for convergence of inputs from the medial prefrontal cortex and lateral habenula on single neurons in the dorsal raphe nucleus. Eur J Neurosci 17, 280-286.

59. Varga, V., Szekely, A. D., Csillag, A., Sharp, T., and Hajos, M., 2001. Evidence for a role of GABA interneurones in the cortical modulation of midbrain 5-hydroxytryptamine neurones. Neuroscience 106, 783-792.

60. Vertes, R. P., 2006. Interactions among the medial prefrontal cortex, hippocampus and midline thalamus in emotional and cognitive processing in the rat. Neuroscience 142, 1-20.

61. Voon, V., Krack, P., Lang, A. E., Lozano, A. M., Dujardin, K., Schupbach, M., D'Ambrosia, J., Thobois, S., Tamma, F., Herzog, J., Speelman, J. D., Samanta, J., Kubu, C., Rossignol, H., Poon, Y. Y., Saint-Cyr, J. A., Ardouin, C., and Moro, E., 2008. A multicentre study on suicide outcomes following subthalamic stimulation for Parkinson's disease. Brain 131, 2720-2728.

62. Wang, R. Y., and Aghajanian, G. K., 1977. Physiological evidence for habenula as major link between forebrain and midbrain raphe. Science 197, 89-91.

63. Wang, S., Zhang, Q. J., Liu, J., Wu, Z. H., Wang, T., Gui, Z. H., Chen, L., and Wang, Y., 2009. Unilateral lesion of the nigrostriatal pathway induces an increase of neuronal firing of the midbrain raphe nuclei 5$\mathrm{HT}$ neurons and a decrease of their response to $5-\mathrm{HT}(1 \mathrm{~A})$ receptor stimulation in the rat. Neuroscience $159,850-861$.

64. Weaver, F. M., Follett, K., Stern, M., Hur, K., Harris, C., Marks, W. J., Jr., Rothlind, J., Sagher, O., Reda, D., Moy, C. S., Pahwa, R., Burchiel, K., Hogarth, P., Lai, E. C., Duda, J. E., Holloway, K., Samii, A., Horn, S., Bronstein, J., Stoner, G., Heemskerk, J., and Huang, G. D., 2009. Bilateral deep brain stimulation vs best medical therapy for patients with advanced Parkinson disease: a randomized controlled trial. JAMA 301, 63-73.

65. Williams, A., Gill, S., Varma, T., Jenkinson, C., Quinn, N., Mitchell, R., Scott, R., Ives, N., Rick, C., Daniels, J., Patel, S., and Wheatley, K., 2010. Deep brain stimulation plus best medical therapy versus best medical therapy alone for advanced Parkinson's disease (PD SURG trial): a randomised, open-label trial. Lancet Neurol 9, 581-591.

66. Zhang, Q. J., Gao, R., Liu, J., Liu, Y. P., and Wang, S., 2007. Changes in the firing activity of serotonergic neurons in the dorsal raphe nucleus in a rat model of Parkinsonos disease. Sheng li xue bao : [Acta physiologica Sinica] 59, 183-189. 



\section{CHAPTER 3}

\section{Cortico-subthalamic projections in the rat}

Janssen MLF, Visser-Vandewalle V, Temel Y

J Exp Clin Med. 2010 Mar;27(1):4-12. 


\section{Abstract}

The subthalamic nucleus (STN) is a key structure in the basal ganglia and plays a major role in the pathogenesis of Parkinson's disease. The STN is a popular target for deep brain stimulation (DBS). DBS of the STN improves motor symptoms. Unfortunately, also negative stimulation induced side-effects on behavior and cognition can occur. These side-effects are thought to be caused by direct stimulation of the associative and limbic pathways that run through the STN. In the primate, three functionally segregated parts are clearly described within the STN: a dorsolateral motor part, a medial limbic part and a ventrolateral associative part. In the rodent however, these subdivisions are not well defined. In this review we describe all anterograde cortico-subthalamic tracer studies to map the rodent STN. As a result, a crude functional subdivision in the rodent STN can be made. The lateral two thirds of the STN receive input from the motor and pre-motor cortex, sparing the medial tip. The medial third receives input from the anterior cingulated, the prelimbic and the agranular insular cortices. There is little evidence for a ventrolateral-dorsomedial subdivision of the medial STN. We conclude that, even though the functional subdivisions are not as clear cut as in the primate STN, a partial anatomical subdivision is present in the rodent STN 


\section{Introduction}

The subthalamic nucleus (STN), also known as Corpus Luysii (Hameleers, et al., 2006), is a key structure in the basal ganglia. This small lentiform nucleus is embryologically a diencephalic structure, but in the adult brain it is located at the diencephalomesencephalic junction (Parent and Hazrati, 1995, Parent and Hazrati, 1995). In spite of its relatively small size, the STN plays a major role in the pathophysiology of Parkinson's disease (PD): the physiological regular firing pattern of STN neurons changes to a pathological bursty neuronal activity (Benazzouz, et al., 2002, Bergman, et al., 1994, Dostrovsky and Lozano, 2002, Magill, et al., 2000, Ni, et al., 2001). The key position of the STN in the basal ganglia and the evident change in its electrical activity in PD, make this nucleus a popular target for neurosurgical therapies such as deep brain stimulation (DBS). Over the last decades, it has been shown consistently that DBS of the STN alleviates motor symptoms and improves quality of life significantly in PD patients (KleinerFisman, et al., 2006, Krack, et al., 2003, Rodriguez-Oroz, et al., 2004, Visser-Vandewalle, et al., 2005). However, DBS of the STN can also produce behavioral side-effects (KleinerFisman, et al., 2006). In a meta-analysis, it has been shown that in up to $40 \%$ of the patients changes in cognition and mood have been observed (Temel, et al., 2006). These changes vary from subtle cognitive changes to major depression with suicidal ideation (Voon, et al., 2008). These behavioral side effects of STN DBS are thought to be caused by direct stimulation of the associative and limbic pathways that run through the STN (Temel, et al., 2005).

In the primate STN, three functional divisions have been circumscribed: a dorsolateral motor part, a medial limbic part and a ventrolateral associative part (Hamani, et al., 2004, Parent and Hazrati, 1995, Temel, et al., 2005). In rodents however, the partition into three separate subdivisions is not entirely clear, despite the fact the rat is often used as an animal model to investigate the motor and non-motor mechanisms of DBS of the STN.

Classically, both the primate and rodent STN receive their input from the globus pallidus externus (GPe), via the multisynaptic cortico-striato-pallido-subthalamic pathway. The primate and rodent STN do not only receive input from basal ganglia nuclei, but they also receive direct afferent input from the cortex. A high density of cortical terminals is present in the STN (Afsharpour, 1985, Canteras, et al., 1990, Carpenter, et al., 1981, Fujimoto and Kita, 1993, Jurgens, 1984, Kitai and Deniau, 1981, Kolomiets, et al., 2001, Kunzle, 1978, Magill, et al., 2004, Monakow, et al., 1978, Nambu, et al., 1996, Nambu, et al., 2000, Nambu, et al., 1997, Nambu, et al., 2002, Rouzaire-Dubois and Scarnati, 1985, Strafella, et al., 2004). These afferent projections from the cortex are also known as the 'hyperdirect' pathway (Nambu, et al., 2002). They arise from several functional cortical areas. The major cortical input to the STN is arising from the motor 
cortex (MC), but also prefrontal cortical areas project directly to the STN. In the primate these cortico-subthalamic loops divide the STN in the three functional subdivisions as mentioned before (Carpenter, et al., 1981, Hamani, et al., 2004, Jurgens, 1984, Kunzle, 1978, Monakow, et al., 1978, Parent and Hazrati, 1995, Temel, et al., 2005). In contrast to the primate, it is not entirely clear how the functional subdivisions are organized in the rat STN.

Here, we have systematically analyzed the studies on the rat cortico-subthalamic projections. Our aim was to describe the functional subdivisions of the rat STN by looking at the origins of the cortical afferents. We only included anterograde tracer studies with injection sites in the cortex. We excluded studies with retrograde tracer injections in the STN, since the tracer can be taken up by neighboring structures and it can diffuse to other STN subdivisions.

\section{Material and Methods}

\section{Search strategy}

A structured Medline (Pubmed) search was performed including articles published up until December 2008. The following key words were used: STN, subthalamic nucleus, subthalamic in combinations with motor cortex (MC), MC, cortex, cortical, cort* and tracing, anterograde, phaseolus, biotinylated dextran amine (BDA), BDA, and rat or rodent. All references in the reviewed articles were checked to find additional studies.

\section{Study Selection}

All studies were reviewed independently by two investigators. Studies were selected according to the following criteria. Only studies with a rodent as a subject were included. We excluded tracing studies in other species, like primates and cats. Studies were included if the injection site was in the cortex and if an anterograde tracer was used. Tracing studies which only made use of retrograde tracing techniques or an injection outside the cortex were excluded. In total 10 studies were selected. The results as well as the figures were systematically and extensively studied by the investigators.

\section{Results}

Projections from the motor and premotor cortical areas

The projection from MC to the STN in the rodent was probably for the first time extensively descrived by Afsharpour (Afsharpour, 1985). In his tracing experiments he injected $0.25-4 \mu \mathrm{l}$ of $3 \mathrm{H}$-proline/3Hleucine or $3 \mathrm{H}$-lycine/3H-leucine using a Hamilton microsyringe according to the cortical parcellation by Hall and Lindholm (Hall and Lindholm, 1974) and Donoghue and Wise (Donoghue and Wise, 1982). Four injections in the ros- 
tral part of the lateral agranular cortex (M1) (coordinates from Bregma: anterioposterior [AP] $5.5 \mathrm{~mm}$, mediolateral [ML] $2.8 \mathrm{~mm}$ until AP $2.3, \mathrm{ML} 4.5 \mathrm{~mm}$, ventrality not provided, animal weights were $230-488 \mathrm{~g}$ ) resulted in tracer signal in the lateral two thirds of the rostral STN with the greatest concentration of the tracer in the dorsolateral tip of the STN (table 1). Injections in the medial part of the lateral agranular cortex (coordinates from Bregma: AP 5.8, ML 1.9 until AP -0.5, ML 3.0, ventrality not provided) resulted in a more band-shaped labeling confined to the ventral aspect of the rostral STN and seemed to shift dorsally at the caudal part of the STN. Injections in the caudal part of the lateral agranular cortex (coordinates from Bregma: AP $-1.0 \mathrm{~mm}$, ML $2.5 \mathrm{~mm}$, ventrality not provided) however, induced only faint labeling in the ventral part of the middle third of the STN. Furthermore, the results showed that the rostral part of the medial agranular cortex (M2) (coordinates from Bregma: AP $+5.8 \mathrm{~mm}$, ML 1.9 until AP $-0.5 \mathrm{~mm}$, $\mathrm{ML} 3.0 \mathrm{~mm}$, ventrality not provided) projected to the ventral two thirds of the medial third of the STN and extended rostrocaudally (fig. 1).

In 1990, micro-electrophoretic injections of wheat germ agglutinin-horseradish peroxidase (WGA-HRP) in the rostral parts of the primary motor cortex (M1) (coordinates: not provided; atlas: not mentioned; animal weights: 170-200g) were performed by Canteras et al. (Canteras, et al., 1990) . Labeling was seen in the rostral two thirds, but not in the ventromedial tip, of the STN. After a more extensive injection in the MC also labeling in the lateral half of the caudal third of the STN was present.

Wan et al. (Wan, et al., 1992) injected leucoagglutinin (PHA-L) into the caudal forelimb region of the MC (coordinates: not provided; atlas: not mentioned; animal weights: 150-300g) by iontophoresis. They found tracer signal in the STN without describing a further topographical organization.

By using iontophoretic injections of WGA-HRP into the orofacial and forelimb areas of $\mathrm{M} 1$, labeling was seen in the more lateral parts of the STN. The orofacial injections (authors used coordinates from the interaural line, re-calculated coordinates from Bregma: AP $+3.5 \mathrm{~mm}, \mathrm{ML} 3.8 \mathrm{~mm}$ and ventral [V] $-1.5 \mathrm{~mm}$ from the cortical surface; atlas: Paxinos and Watson, edition 2, 1986; animal weights: 270-300g) resulted in tracer signal in the central part of the mediolateral extension of the STN, whereas injections in the forelimb area of M1 (re-calculated coordinates from Bregma: AP +3.0mm, ML 2.3mm and $\mathrm{V}-1.5 \mathrm{~mm}$ from the cortical surface) projected more ventrally and caudally, sparing the medial and lateral parts of the STN (Kolomiets, et al., 2001, Paxinos and Watson, 1986).

In a more recent study, Degos et al. (Degos, et al., 2008) injected iontophoretically PHA-L in the orofacial motor area of M1 (authors used coordinates from the interaural line, re-calculated coordinates from Bregma: $A P+3.5 \mathrm{~mm}, \mathrm{ML}+3.8 \mathrm{~mm}$ and $\mathrm{V}-1.2 \mathrm{~mm}$; atlas: Paxinos and Watson, edition 2 (Paxinos and Watson, 1986); animal weights: 300$360 \mathrm{~g})$. Tracer signal was seen in the caudal two thirds of the STN, in the lateral part of 
the medial third of the STN and more to the medial side in the caudal third. The ventromedial tip remained free of tracer.

Afsharpour 1985
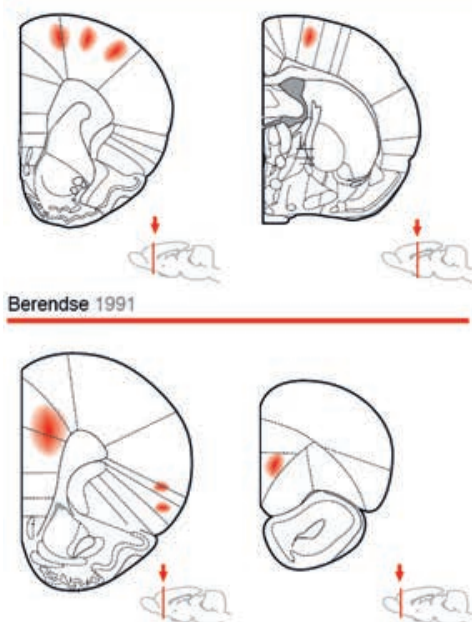

Canteras 1990
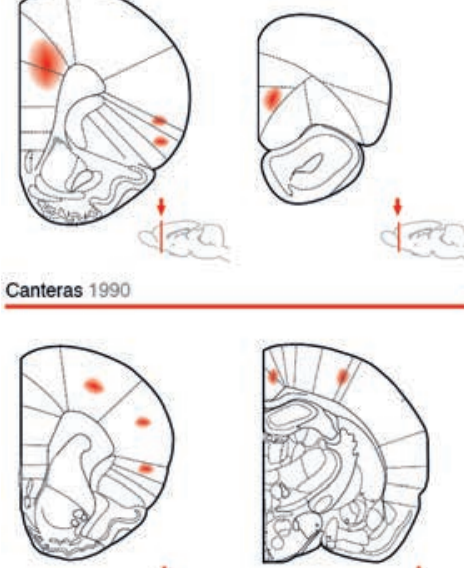

बan

Kolomiets 2001

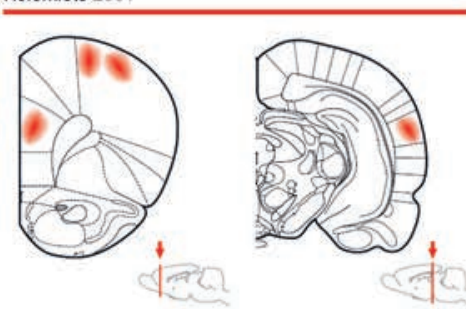

Orieux 2002

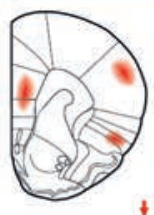

क्ये

Tsumori 2006

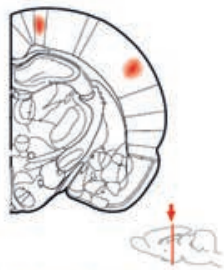

Canteras 1988

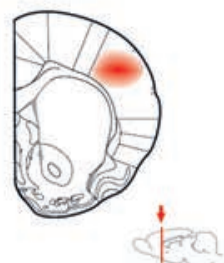

Degos 2008

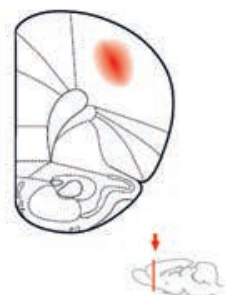

Leichnetz 1987

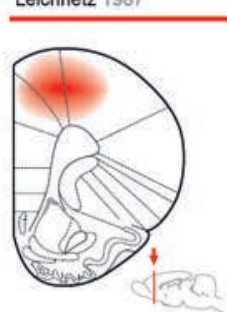

Wan 1992

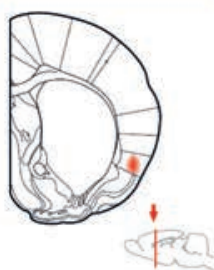

Fig. 1. Overview of the estimated injection or implantation sites in the different cortical regions of the rat. The red areas represent the injection sites in coronal sections of the brain. The red line in the sagital sections shows the estimated anteroposterior level of the coronal section. 
Projections from the prelimbic cortex

Only in a few anatomical studies an anterograde tracer was injected into the prelimbic cortex in order to determine the projection to the STN. The oldest publication which we have found about the projections from the prelimbic cortex to the STN showed tracer signal in the medial STN (Leichnetz, et al., 1987). The authors used a pellet of HRP-gel. The pellet was implanted in the dorsomedial frontal shoulder cortex (coordinates: not provided; atlas: not mentioned; animal weights: not provided), which encompasses the medial precentral and anterior cingulate cortices. The anatomical data showed heavy labeling in the dorsomedial STN.

A study by Berendse and Groenewegen (Berendse and Groenewegen, 1991) using PHA-L as an anterograde tracer showed labeling in the caudal part of the most medial part of the STN after pressure injections in the dorsal part of the prelimbic cortex (coordinates from Bregma: not provided; atlas: not mentioned; animal weights: not provided). After injection in the ventral part of the prelimbic cortex (coordinates from Bregma: not provided), tracer signal was only visualized in the adjacent LH and not in the STN.

Kolomiets et al. (Kolomiets, et al., 2001) injected WGA-HRP into the prelimbic medial orbital areas of the prefrontal cortex (authors used coordinates from the interaural line, re-calculated coordinates from Bregma: AP $+3.5 \mathrm{~mm}, \mathrm{ML} 0.4 \mathrm{~mm}$ and $V-3.5 \mathrm{~mm}$ from the cortical surface; atlas: Paxinos and Watson, edition 2, (Paxinos and Watson, 1986); animal weights: 270-300g) using iontophoresis. The anterograde tracer was only present in the medial third of the rat STN.

Later, Orieux et al (Orieux, et al., 2002) showed scattered positive fibers in the medial part of the STN. Fibers were seen in almost the entire anteroposterior extent of the medial STN. The authors injected BDA in the prelimbic/ medial orbital cortex according to us. This is in contrast to the opinion of the author himself who stated that the iontophoresis was in the cingulate cortex (coordinates from Bregma: $\mathrm{AP}+3.5 \mathrm{~mm}, \mathrm{ML}$ $0.5 \mathrm{~mm}$ and $\mathrm{V}-3.0 \mathrm{~mm}$ from the cortical surface; atlas: Paxinos and Watson, edition 4, (Paxinos and Watson, 1998); animal weights: 250-300g).

\section{Projections from the anterior cingulate cortex}

One of the et al. (Leichnetz, et al., 1987). The pellet of HRP-gel in the dorsomedial shoulder cortex (coordinates: not provided; atlas: not mentioned; animal weights: not provided) resulted in tracer signal in the medial STN.

A study using PHA-L demonstrated labeling of the ventral and lateral parts of the medial STN, most densely at the caudal levels. The tracer was injected with a pump into the dorsal anterior cingulate cortex (coordinates: not provided; atlas: not mentioned; animal weights: not provided) (Berendse and Groenewegen, 1991). 


\section{Projections from the insular cortex}

Canteras et al. (Canteras, et al., 1990). injected WGA-HRP into the granular insular cortex using micro-electrophoretic deposits and found small varicosities in the dorsomedial narrow strip of the STN (coordinates from Bregma: not provided; atlas: not mentioned; animal weights: 170-200g).

A study by Berendse and Groenewegen (Berendse and Groenewegen, 1991) described the afferent projections after injection of PHA-L into the dorsal agranular insular and the ventral agranular insular cortices (coordinates: not provided; atlas: not provided; animal weights: not provided). Tracer signal was only seen in the lateral hypothalamic area, but not in the STN, after injection in the ventral agranular insular cortex. In contrast, fibers were seen in the rostral part of the STN in animals with an injection of BDA into the dorsal agranular cortex.

An iontophoretic deposit of BDA the dorsal agranular insular cortex (coordinates from Bregma: $\mathrm{AP}+2.0 \mathrm{~mm}, \mathrm{ML} 5.0 \mathrm{~mm}$ and $\mathrm{V}-4.5 \mathrm{~mm}$ from the cortical surface; atlas: Paxinos and Watson, edition 4, (Paxinos and Watson, 1998); animal weight: 250-300g) induced weak labeling in the anterior portion of the STN, occupying the entire mediolateral extent (Orieux, et al., 2002).

A third study with an injection of BDA into the insular cortex (coordinates from Bregma: AP $0.0--0.5 \mathrm{~mm}, \mathrm{ML} 5.6-6.0 \mathrm{~mm}$ and $\mathrm{V}-7.0--7.6 \mathrm{~mm}$; atlas: Paxinos and Watson, edition 5, (Paxinos and Watson, 2005); animal weight: 230-300g) using iontophoresis showed no tracer in the STN (Tsumori, et al., 2006).

\section{Projections from the somatosensory cortex}

Afsharpour (Afsharpour, 1985) was one of the first to look at the projections from the somatosensory cortex in the rat. He injected the $3 \mathrm{H}$-proline/3H-leucine or $3 \mathrm{H}$ lycine/3H-leucine tracer into the caudal granular somatosensory cortex according to the cortical parcellation by Hall and Lindholm (Hall and Lindholm, 1974) and Donoghue and Wise (Donoghue and Wise, 1982) (coordinates from Bregma: AP -2.5mm, ML 6.0mm, ventrality not provided; animal weighst: $230-488 \mathrm{~g})$. In his experiment there was no labeling in the STN. However, Canteras et al. (Canteras, et al., 1988, Canteras, et al., 1990) injected WGA-HRP iontophoretically into the rostral half of the primary somatosensory cortex (coordinates: not provided; atlas: not mentioned; animal weights: 170$200 \mathrm{~g}$ ) and found positive fibers in the dorsolateral part at the midrostrocaudal level of the STN, but also with a lesser density in the dorsal part of the rostral portion and the dorsolateral tip of the caudal district. In a follow-up experiment they injected WGA-HRP into the caudal half of the primary somatosensory cortex (coordinates: not provided; atlas: not provided; animal weights: 170-200g) using iontophoresis and found only few, if any, labeling in the STN (Canteras, et al., 1990). In 2002, for a second time a tracer study with BDA did not show any labeling in the STN after iontophoretic injection of the 
dye into somatosensory cortex (coordinates from Bregma: AP $+1.0 \mathrm{~mm}, \mathrm{ML} 4.5 \mathrm{~mm}$ and $V-3.2 \mathrm{~mm}$ from the cortical surface; atlas: Paxinos and Watson, edition 4, (Paxinos and Watson, 1998); animal weights: 250-300g) (Orieux, et al., 2002).

\section{Projections from other cortical areas}

Some studies have investigated the afferent input to STN from other cortical areas. No labeling was found in the STN after tracer injections in the retrosplenial cortex, and primary visual and primary auditory cortices (Canteras, et al., 1990, Kolomiets, et al., 2001). Again no STN labeling was found after injections of PHA-L into the medial orbital and infralimbic cortex (Berendse and Groenewegen, 1991).

Table 1. In the table on the next pages an overview is given of all the injection sites per author, with the corresponding tracing site in the subthalamic nucleus. The anatomical description of the injection sites and the (re-calculated) coordinates from Bregma are shown if provided. Also the technique and tracer used are given. anteroposterior (AP), mediolateral $(\mathrm{ML})$, ventrality $(\mathrm{V})$, ventrality measured from the cortex (c). *Anatomical injection site based on figure in original article. 


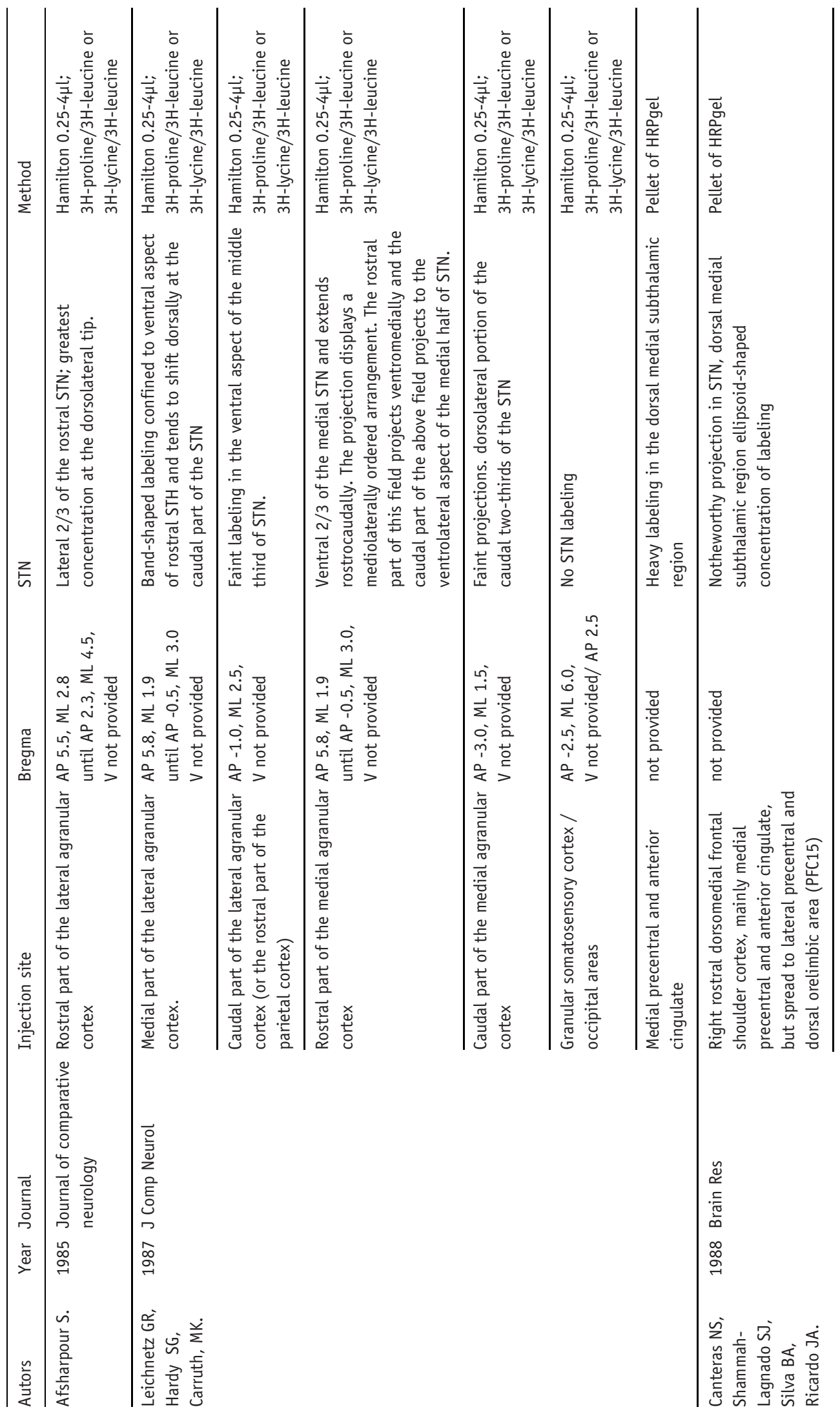




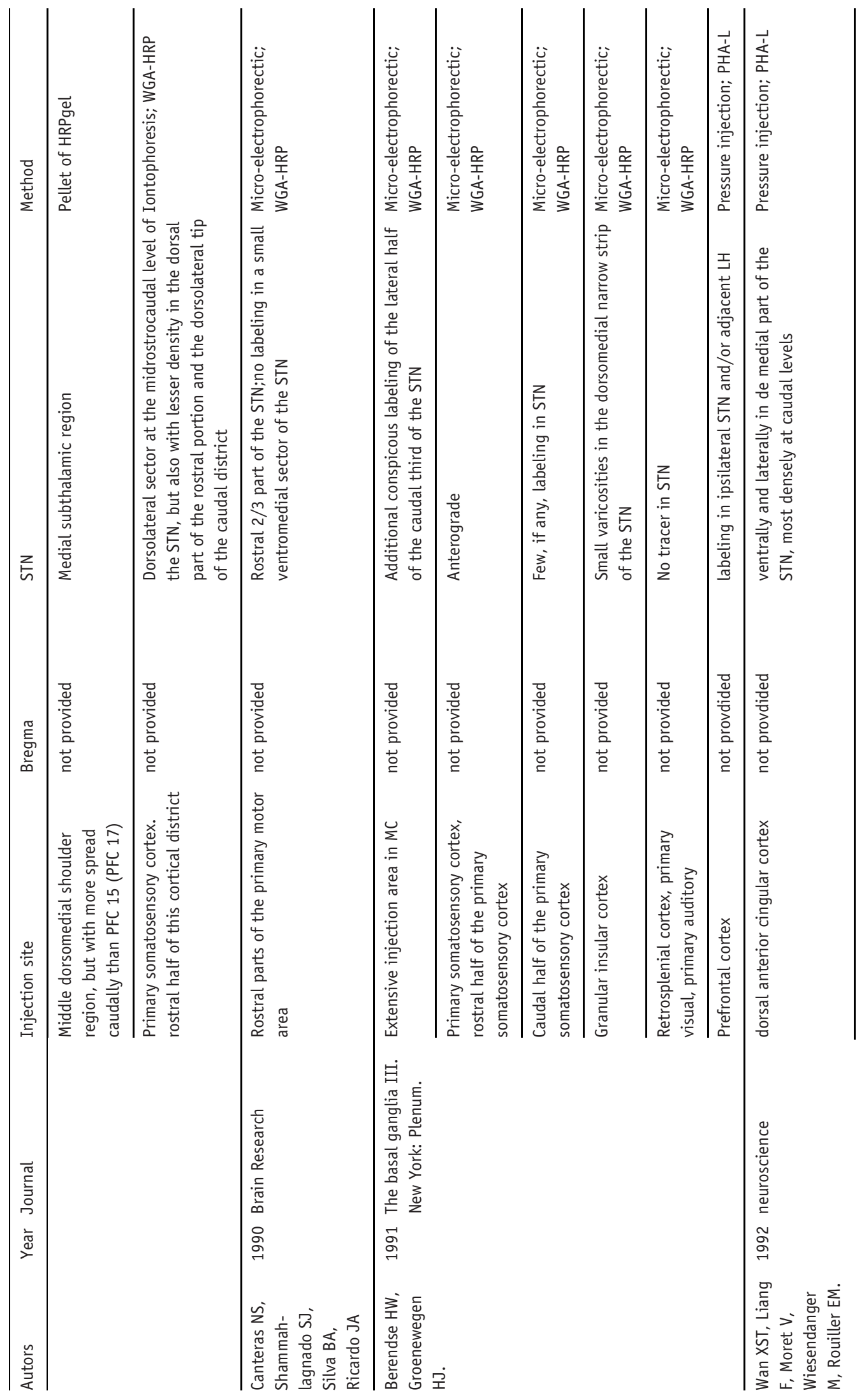




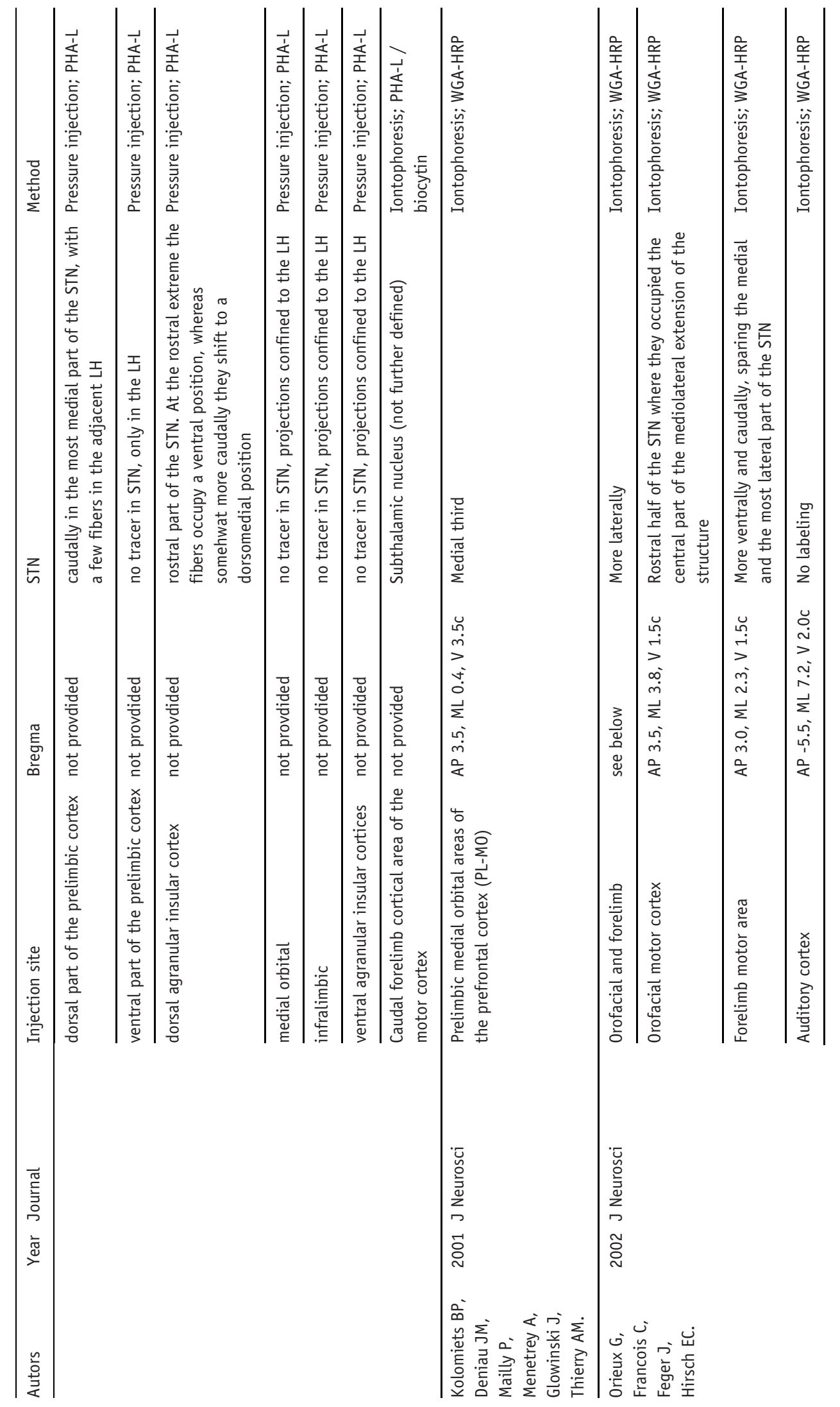




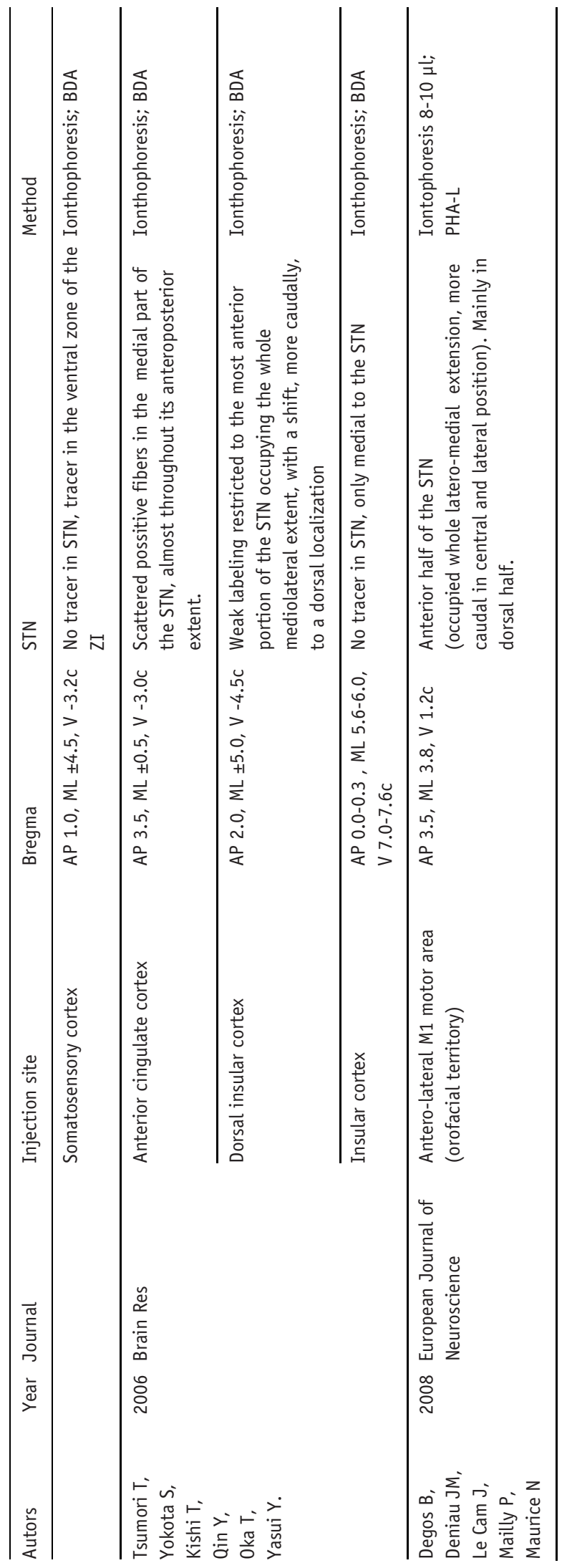




\section{Discussion}

The functional subdivisions of the rat STN

The reviewed data demonstrate the existence of a rough subdivision system in the rat STN. Several parallel projections from the cortex to the rodent STN are present. The most important projections originate from the motor and premotor cortex (Afsharpour, 1985, Canteras, et al., 1990, Degos, et al., 2008, Kolomiets, et al., 2001, Wan, et al., 1992), cingulate cortex (Berendse and Groenewegen, 1991, Leichnetz, et al., 1987, Orieux, et al., 2002), prelimbic (Berendse and Groenewegen, 1991, Kolomiets, et al., 2001, Leichnetz, et al., 1987), and the agranular insular cortex (Berendse and Groenewegen, 1991, Orieux, et al., 2002). Afferent fibers from the motor cortex and the premotor cortex project to the lateral two thirds of the rat STN, thereby sparing the medial tip. The medial third of the STN receives input from the anterior cingulate, the prelimbic and the agranular insular cortices. There is little evidence for a ventrolateraldorsomedial subdivision of the medial STN (Berendse and Groenewegen, 1991). The ventrolateral division of the medial third receives its input mainly from the anterior cingulate cortex, whereas the projection from the pre-limbic and agranular insular cortex is restricted to the dorsomedial part (fig. 2).

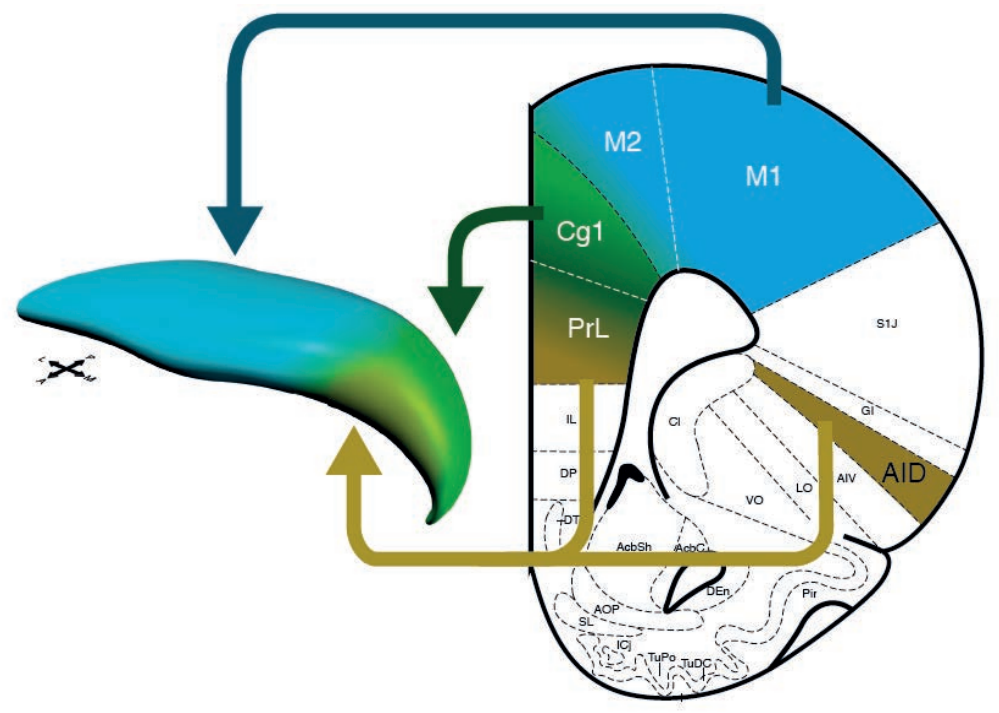

Fig. 2. This figure illustrates the functional subdivisions of the rodent STN. The nucleus has anatomically two major subdivisions: the lateral two-thirds consist of the motor part (blue) and the medial third is the limbic/ associative part (green/yellow). These divisions are not strictly segregated but partially overlapping. Thereby small evidence is present for an anatomical organization of the medial part. The arrows show the cortical projections to the different subthalamic subdivisions. 


\section{Differences between the studies}

There are some evident differences in the results between the various anatomical studies. They may result from the variances in the method of injection, injection sites and tracers used. We will elaborate on the main dissimillarities found per cortical injection site.

Berendse and Groenewegen (Berendse and Groenewegen, 1991) only observed labeling in the dorso-medial part of the STN after injection in the cingulate gyrus, though Leichnetz et al. (Leichnetz, et al., 1987) found tracer in the whole medial STN. The findings of Leichnetz et al. (Leichnetz, et al., 1987) could be due to the use of relatively big pellets, making their method less accurate.. A high-quality methodological tracer study is needed to see whether the cingulate gyrus projects to the entire medial STN or that its projection is strictly focused to the dorso-medial part of the medial STN.

Another discrepancy is present between the studies about the projections from the prelimbic cortex to the medial STN. Kolomiets et al. (Kolomiets, et al., 2001) showed tracing to the medial part of STN from the dorsomedial frontal shoulder cortex involving the prelimbic, but also the cingulate cortex. The injection of Orieux et al. (Orieux, et al., 2002 ) in the medial prefrontal cortex, according to us mainly in the prelimbic and medial orbital cortices, gave labeling throughout the whole anteroposterior extent of the medial STN. This was in contradiction with the iontophoretic study of Berendse and Groenewegen (Berendse and Groenewegen, 1991) who only found labeling in the dorsomedial part.

The dorsal part of the agranular insular cortex seems to project to the dorsomedial part of the STN. Canteras et al. (Canteras, et al., 1990) described this after injection into the granular cortex, and both Berendse and Groenewegen (Berendse and Groenewegen, 1990) as well as Orieux et al. (Orieux, et al., 2002) showed the same after an injection into the dorsal agranular insular cortex. Tsumori et al. (Tsumori, et al., 2006)did not observe a projection from the insular cortex. This implicates that the results from Canteras et al. (Canteras, et al., 1990) may perhaps be a result of diffusion of the tracer to the dorsal agranular cortex. The ventral part of the agranular insular cortex also doesn't seem to project to the STN, but only to the adjacent LH.

There is only little evidence that the somatosensory cortex projects to the STN. Canteras et al. (Canteras, et al., 1988) found some projections from the caudal half of the somatosensory cortex but this is in contradiction to the findings of Afsharpour (Afsharpour, 1985) and Orieux (Orieux, et al., 2002). The findings of Canteras et al. (Canteras, et al., 1988) could again be due to leakage of the tracer to the motor cortical areas. 


\section{Similarities between the studies}

There were also evident consistencies. For instance, injections in the motor areas never resulted in labeling in the medial part of the STN. Only Afsharpour (Afsharpour, 1985) mentioned labeling in the medial part of the STN after injection in the agranular cortex. If looked carefully to the injection sites of this study, diffusion of the tracer to the prefrontal areas like the cingulate gyrus cannot be ruled out.

Another challenge encountered in this review was the fact that some authors used different descriptions for the same anatomical site and did not always provide the stereotactic coordinates and relevant images.

\section{Correlation between the cortico-subthalamic and subcortico-subthalamic projections}

The topography of the rat STN based on the cortico-subthalamic projections matches with the input from the subcortical regions. The lateral STN receives input from the lateral part of the parafascicular thalamic nucleus (Groenewegen and Berendse, 1990, Sugimoto, et al., 1983), which also projects to the lateral part of the caudate putamen (CPu). The lateral CPu indirectly projects to the lateral STN via the lateral part of the globus pallidus (GP: equivalent of the GPe in primates) (Gerfen, 1985, Kita and Kitai, 1987, Ricardo, 1980). The medial part of the STN receives its input from the most medial part of the parafascicular thalamic nucleus, which also projects to the nucleus accumbens (NAc) and the medial CPu. The NAc and the medial CPu project indirectly to the medial STN via respectively the lateral part of the subcommisural ventral pallidum and the medial part of the GP (Berendse and Groenewegen, 1990, Maurice, et al., 1998). The NAc, in turn, receives input from the prefrontal cortex. It seems that the functionally different cortical areas project both in a 'hyperdirect' and indirect way to the STN in a parallel organized manner. Moreover, the efferent output of the STN to the GP, entepeduncular nucleus (EP: equivalent of the GPi in primates) and the ventral pallidum matches with the subdivisions based on the cortico-subthalamic tracer studies. The lateral STN mainly projects to the lateral GP and EP, whereas the medial part projects to the ventral pallidum and the medial GP and EP in the rat (Groenewegen and Berendse, 1990, Kita and Kitai, 1987, Maurice, et al., 1998).

\section{Cortical afferents}

Our findings from the reviewed data suggest that the cortical projections to the rat STN follow a specific pattern of innervation. The major input arises from the motor and premotor areas. The afferent projections from the motor cortex arise mainly from layer V (Gradinaru, et al., 2009, Kitai and Deniau, 1981) and are collaterals of the pyramidal tract or from fibers that also project to the striatum. In addition, projections arise from the prelimbic (Berendse and Groenewegen, 1991, Kolomiets, et al., 2001, Leichnetz, et al., 1987), anterior cingulate (Berendse and Groenewegen, 1991, Leichnetz, et al., 1987, 
Orieux, et al., 2002) and dorsal agranular insular cortex (Berendse and Groenewegen, 1991, Orieux, et al., 2002). The terminals of the cortical axons make contact with small dendrites and cell bodies of STN neurons and use glutamate as neurotransmitter.

The rat STN does not receive input from the ventral agranular and the granular insular, retrosplenial, primary visual and primary auditory, medial orbital and infralimbic cortices and probably also not from the somatosensenory cortex. Also noticeable is that not all cortical areas which are involved in associative and/ or limbic functions project directly to the STN.

Integration of the motor, limbic and associative projections in the STN

In agreement with the anatomical tracing studies, electrophysiological studies demonstrate a functional subdivision as well. Neurons that respond to cortical stimulation were found in the anatomically defined territories of the STN (Kolomiets, et al., 2001, Magill, et al., 2004, Maurice, et al., 1998). Nevertheless, it seems unlikely that the subdivisions of the rat STN are entirely segregated from each other. The rat STN has a higher number of neurons per cubic millimeter $\left(30,000\right.$ cells per $\left.\mathrm{mm}^{3}\right)$ compared to the primate and human STN (2,300 cells per $\mathrm{mm}^{3}$ ) (Hardman, et al., 2002). Moreover, the dendrites can extend across almost the entire STN. (Heimer, et al., 1995) An extra argument is that a 'hyperdirect' response to stimulation of the prelimbic cortex is seen in $7 \%$ of the STN cells which also respond to motor cortex stimulation (Kolomiets, et al., 2001). Despite these noteworthy differences between the rat STN and the (human) primate STN the internal organization and its place in the basal ganglia is highly comparable.

\section{Limitations and methodological considerations}

Only ten studies could be identified in which an anterograde tracer was injected in a cortical area and the tracer signal was analysed in the STN. Due to the low number of studies, we also included papers in which the neuroanatomical tracing of the corticosubthalamic projection was only a part of a broader study.

Various tracing techniques have been used in the studies reviewed here. Several authors have used tritiated amino acids or WGA-HRP. By using these tracers, it is not possible to distinguish between terminating or passing fibers. Others have used BDA which gives a very good labeling, but can be taken up by passing fibers or transported retrogradely. PHA-L has also been used and is a very specific anterograde tracer (Reiner, et al., 2000). The variety of techniques used, complicates the comparison between the studies. Therefore, we think that the results of the individual studies must be handled with care.

Another factor that needs to be taken into account is that the nomenclature of the different cortical areas has been amended over time, based on new insights. The ana- 
tomical names and delineations of the cortical regions have therefore changed in the newer editions of the atlas of Paxinos and Watson (Paxinos and Watson, 2005). In the reviewed studies, we have relied on the authors' description of the injection sites and projections to the STN, since detailed anatomical pictures were often lacking.

\section{Conclusion}

A partial anatomical subdivision system is present in the rodent STN, although it is not as clear cut as in the primate. Neurons in the medial STN mainly get their afferent input from the limbic and associative cortical areas and those in the lateral two thirds receive their input from the motor areas.

\section{Acknowledgements}

The authors gratefully acknowledge the support of the BrainGain Smart Mix Programme of the Netherlands Ministry of Economic Affairs and the Netherlands Ministry of Education, Culture and Science (grant no.: SSM06011) and the Netherlands Organisation for Scientific Research (NWO-Veni grant to author). 


\section{References}

1. Afsharpour, S., 1985. Topographical projections of the cerebral cortex to the subthalamic nucleus. J Comp Neurol 236, 14-28.

2. Benazzouz, A., Breit, S., Koudsie, A., Pollak, P., Krack, P., and Benabid, A. L., 2002. Intraoperative microrecordings of the subthalamic nucleus in Parkinson's disease. Mov Disord 17 Suppl 3, S145-149.

3. Berendse, H. W., and Groenewegen, H. J., 1990. Organization of the thalamostriatal projections in the rat, with special emphasis on the ventral striatum. J Comp Neurol 299, 187-228.

4. Berendse, H. W., and Groenewegen, H. J., 1991. The connections of the medial part of the subthalamic nucleus in the rat: evidence for a parallel organization. The basal ganglia III. New York: Plenum., 89-98.

5. Bergman, H., Wichmann, T., Karmon, B., and DeLong, M. R., 1994. The primate subthalamic nucleus. II. Neuronal activity in the MPTP model of parkinsonism. J Neurophysiol 72, 507-520.

6. Canteras, N. S., Shammah-Lagnado, S. J., Silva, B. A., and Ricardo, J. A., 1988. Somatosensory inputs to the subthalamic nucleus: a combined retrograde and anterograde horseradish peroxidase study in the rat. Brain Res 458, 53-64.

7. Canteras, N. S., Shammah-Lagnado, S. J., Silva, B. A., and Ricardo, J. A., 1990. Afferent connections of the subthalamic nucleus: a combined retrograde and anterograde horseradish peroxidase study in the rat. Brain Res 513, 43-59.

8. Carpenter, M. B., Carleton, S. C., Keller, J. T., and Conte, P., 1981. Connections of the subthalamic nucleus in the monkey. Brain Res 224, 1-29.

9. Degos, B., Deniau, J. M., Le Cam, J., Mailly, P., and Maurice, N., 2008. Evidence for a direct subthalamocortical loop circuit in the rat. Eur J Neurosci 27, 2599-2610.

10. Donoghue, J. P., and Wise, S. P., 1982. The motor cortex of the rat: cytoarchitecture and microstimulation mapping. J Comp Neurol 212, 76-88.

11. Dostrovsky, J. O., and Lozano, A. M., 2002. Mechanisms of deep brain stimulation. Mov Disord 17 Suppl 3, s63-68.

12. Fujimoto, K., and Kita, H., 1993. Response characteristics of subthalamic neurons to the stimulation of the sensorimotor cortex in the rat. Brain Res 609, 185-192.

13. Gerfen, C. R., 1985. The neostriatal mosaic. I. Compartmental organization of projections from the striatum to the substantia nigra in the rat. J Comp Neurol 236, 454-476.

14. Gradinaru, V., Mogri, M., Thompson, K. R., Henderson, J. M., and Deisseroth, K., 2009. Optical Deconstruction of Parkinsonian Neural Circuitry. Science.

15. Groenewegen, H. J., and Berendse, H. W., 1990. Connections of the subthalamic nucleus with ventral striatopallidal parts of the basal ganglia in the rat. J Comp Neurol 294, 607-622.

16. Hall, R. D., and Lindholm, E. P., 1974. Organization of the motor and somatosensory neocortex in the albino rat. . Brain Res. 66, 23-38.

17. Hamani, C., Saint-Cyr, J. A., Fraser, J., Kaplitt, M., and Lozano, A. M., 2004. The subthalamic nucleus in the context of movement disorders. Brain 127, 4-20.

18. Hameleers, R., Temel, Y., and Visser-Vandewalle, V., 2006. History of the corpus luysii: 1865-1995. Arch Neurol 63, 1340-1342.

19. Hardman, C. D., Henderson, J. M., Finkelstein, D. I., Horne, M. K., Paxinos, G., and Halliday, G. M., 2002. Comparison of the basal ganglia in rats, marmosets, macaques, baboons, and humans: volume and neuronal number for the output, internal relay, and striatal modulating nuclei. J Comp Neurol 445, 238255.

20. Heimer, L., Alheid, G. F., and Zahm, D. S., 1995. Basal ganglia. In: Paxinos, G., (Ed., The Rat Nervous System. Academic Press Inc., San Diego, pp. 579-628.

21. Jurgens, U., 1984. The efferent and afferent connections of the supplementary motor area. Brain Res 300, 63-81.

22. Kita, H., and Kitai, S. T., 1987. Efferent projections of the subthalamic nucleus in the rat: light and electron microscopic analysis with the PHA-L method. J Comp Neurol 260, 435-452. 
23. Kitai, S. T., and Deniau, J. M., 1981. Cortical inputs to the subthalamus: intracellular analysis. Brain Res 214, 411-415.

24. Kleiner-Fisman, G., Herzog, J., Fisman, D. N., Tamma, F., Lyons, K. E., Pahwa, R., Lang, A. E., and Deuschl, G., 2006. Subthalamic nucleus deep brain stimulation: summary and meta-analysis of outcomes. Mov Disord 21 Suppl 14, S290-304.

25. Kolomiets, B. P., Deniau, J. M., Mailly, P., Menetrey, A., Glowinski, J., and Thierry, A. M., 2001. Segregation and convergence of information flow through the cortico-subthalamic pathways. J Neurosci 21, 5764-5772.

26. Krack, P., Batir, A., Van Blercom, N., Chabardes, S., Fraix, V., Ardouin, C., Koudsie, A., Limousin, P. D., Benazzouz, A., LeBas, J. F., Benabid, A. L., and Pollak, P., 2003. Five-year follow-up of bilateral stimulation of the subthalamic nucleus in advanced Parkinson's disease. N Engl J Med 349, 1925-1934.

27. Kunzle, H., 1978. An autoradiographic analysis of the efferent connections from premotor and adjacent prefrontal regions (areas 6 and 9) in macaca fascicularis. Brain Behav Evol 15, 185-234.

28. Leichnetz, G. R., Hardy, S. G., and Carruth, M. K., 1987. Frontal projections to the region of the oculomotor complex in the rat: a retrograde and anterograde HRP study. J Comp Neurol 263, 387-399.

29. Magill, P. J., Bolam, J. P., and Bevan, M. D., 2000. Relationship of activity in the subthalamic nucleusglobus pallidus network to cortical electroencephalogram. J Neurosci 20, 820-833.

30. Magill, P. J., Sharott, A., Bevan, M. D., Brown, P., and Bolam, J. P., 2004. Synchronous unit activity and local field potentials evoked in the subthalamic nucleus by cortical stimulation. J Neurophysiol 92, 700714.

31. Maurice, N., Deniau, J. M., Glowinski, J., and Thierry, A. M., 1998. Relationships between the prefrontal cortex and the basal ganglia in the rat: physiology of the corticosubthalamic circuits. J Neurosci 18, 95399546.

32. Monakow, K. H., Akert, K., and Kunzle, H., 1978. Projections of the precentral motor cortex and other cortical areas of the frontal lobe to the subthalamic nucleus in the monkey. Exp Brain Res 33, 395-403.

33. Nambu, A., Takada, M., Inase, M., and Tokuno, H., 1996. Dual somatotopical representations in the primate subthalamic nucleus: evidence for ordered but reversed body-map transformations from the primary motor cortex and the supplementary motor area. J Neurosci 16, 2671-2683.

34. Nambu, A., Tokuno, H., Hamada, I., Kita, H., Imanishi, M., Akazawa, T., Ikeuchi, Y., and Hasegawa, N., 2000. Excitatory cortical inputs to pallidal neurons via the subthalamic nucleus in the monkey. J Neurophysiol 84, 289-300.

35. Nambu, A., Tokuno, H., Inase, M., and Takada, M., 1997. Corticosubthalamic input zones from forelimb representations of the dorsal and ventral divisions of the premotor cortex in the macaque monkey: comparison with the input zones from the primary motor cortex and the supplementary motor area. Neurosci Lett 239, 13-16.

36. Nambu, A., Tokuno, H., and Takada, M., 2002. Functional significance of the cortico-subthalamo-pallidal 'hyperdirect' pathway. Neurosci Res 43, 111-117.

37. Ni, Z. G., Bouali-Benazzouz, R., Gao, D. M., Benabid, A. L., and Benazzouz, A., 2001. Time-course of changes in firing rates and firing patterns of subthalamic nucleus neuronal activity after 6-OHDA-induced dopamine depletion in rats. Brain Res 899, 142-147.

38. Orieux, G., Francois, C., Feger, J., and Hirsch, E. C., 2002. Consequences of dopaminergic denervation on the metabolic activity of the cortical neurons projecting to the subthalamic nucleus in the rat. J Neurosci 22, 8762-8770.

39. Parent, A., and Hazrati, L. N., 1995. Functional anatomy of the basal ganglia. I. The cortico-basal gangliathalamo-cortical loop. Brain Res Brain Res Rev 20, 91-127.

40. Parent, A., and Hazrati, L. N., 1995. Functional anatomy of the basal ganglia. II. The place of subthalamic nucleus and external pallidum in basal ganglia circuitry. Brain Res Brain Res Rev 20, 128-154.

41. Paxinos, G., and Watson, C., 1986. The Rat Brain in Stereotaxic Coordinates. Acadmic Press, New York.

42. Paxinos, G., and Watson, C., 1998. The Rat Brain in Stereotaxic Coordinates. Academic Press, New York.

43. Paxinos, G., and Watson, C., 2005. The Rat Brain in Stereotaxic Coordinates Academic Press, New York. 
44. Reiner, A., Veenman, C. L., Medina, L., Jiao, Y., Del Mar, N., and Honig, M. G., 2000. Pathway tracing using biotinylated dextran amines. J Neurosci Methods 103, 23-37.

45. Ricardo, J. A., 1980. Efferent connections of the subthalamic region in the rat. I. The subthalamic nucleus of Luys. Brain Res 202, 257-271.

46. Rodriguez-Oroz, M. C., Zamarbide, I., Guridi, J., Palmero, M. R., and Obeso, J. A., 2004. Efficacy of deep brain stimulation of the subthalamic nucleus in Parkinson's disease 4 years after surgery: double blind and open label evaluation. J Neurol Neurosurg Psychiatry 75, 1382-1385.

47. Rouzaire-Dubois, B., and Scarnati, E., 1985. Bilateral corticosubthalamic nucleus projections: an electrophysiological study in rats with chronic cerebral lesions. Neuroscience 15, 69-79.

48. Strafella, A. P., Vanderwerf, Y., and Sadikot, A. F., 2004. Transcranial magnetic stimulation of the human motor cortex influences the neuronal activity of subthalamic nucleus. Eur J Neurosci 20, 2245-2249.

49. Sugimoto, T., Hattori, T., Mizuno, N., Itoh, K., and Sato, M., 1983. Direct projections from the centre median-parafascicular complex to the subthalamic nucleus in the cat and rat. J Comp Neurol 214, 209216.

50. Temel, Y., Blokland, A., Steinbusch, H. W., and Visser-Vandewalle, V., 2005. The functional role of the subthalamic nucleus in cognitive and limbic circuits. Prog Neurobiol 76, 393-413.

51. Temel, Y., Kessels, A., Tan, S., Topdag, A., Boon, P., and Visser-Vandewalle, V., 2006. Behavioural changes after bilateral subthalamic stimulation in advanced Parkinson disease: a systematic review. Parkinsonism Relat Disord 12, 265-272.

52. Tsumori, T., Yokota, S., Kishi, T., Qin, Y., Oka, T., and Yasui, Y., 2006. Insular cortical and amygdaloid fibers are in contact with posterolateral hypothalamic neurons projecting to the nucleus of the solitary tract in the rat. Brain Res 1070, 139-144.

53. Visser-Vandewalle, V., van der Linden, C., Temel, Y., Celik, H., Ackermans, L., Spincemaille, G., and Caemaert, J., 2005. Long-term effects of bilateral subthalamic nucleus stimulation in advanced Parkinson disease: a four year follow-up study. Parkinsonism Relat Disord 11, 157-165.

54. Voon, V., Krack, P., Lang, A. E., Lozano, A. M., Dujardin, K., Schupbach, M., D'Ambrosia, J., Thobois, S., Tamma, F., Herzog, J., Speelman, J. D., Samanta, J., Kubu, C., Rossignol, H., Poon, Y. Y., Saint-Cyr, J. A., Ardouin, C., and Moro, E., 2008. A multicentre study on suicide outcomes following subthalamic stimulation for Parkinson's disease. Brain 131, 2720-2728.

55. Wan, X. S., Liang, F., Moret, V., Wiesendanger, M., and Rouiller, E. M., 1992. Mapping of the motor pathways in rats: c-fos induction by intracortical microstimulation of the motor cortex correlated with efferent connectivity of the site of cortical stimulation. Neuroscience 49, 749-761. 



\section{CHAPTER 4}

\section{The antidepressant effects of ventromedial prefrontal cortex is associated with neural activation in the medial part of the subthalamic nucleus}

Janssen MLF, Lim LW, Kocabicak E, Temel Y

Adapted from Lim LW, Janssen MLF, Kocabicak E, Temel Y

Accepted in Behavioural Brain Research 


\section{Abstract}

The nucleus accumbens (NAc), ventromedial prefrontal cortex (VmPFC), and cingulate gyrus $(\mathrm{Cg})$ are key regions in the control of mood-related behaviors. Electrical stimulation of these areas induces antidepressant-like effects in both patients and animal models. Another structure whose limbic connections are receiving more interest in the context of mood-related behaviors is the medial part of the subthalamic nucleus (STN). Here, we tested the hypothesis that the mood-related effects of NAc, vmPFC, and $\mathrm{Cg}$ are accompanied by changes in the neural activity of the STN. We performed highfrequency stimulation (HFS) of the NAc, vmPFC, and Cg. Animals were behaviorally tested for hedonia and forced swim immobility; and the neuronal activities in the different parts of the STN were assessed by means of c-Fos immunoreactivity (c-Fos-ir). Our results showed that HFS of the NAc and VMPFC, but not Cg reduced anhedonic-like and forced swim immobility behaviors. Interestingly, there was a significant increase of c-Fos-ir in the medial STN with HFS of the VMPFC, but not the NAC and Cg as compared to the sham. Correlation analysis showed that the medial STN is associated with the antidepressant-like behaviors in vmPFC HFS animals. No behavioral correlation was found with respect to behavioural outcome and activity in the lateral STN. In conclusion, HFS of the VmPFC induced profound antidepressant-like effects with enhanced neural activity in the medial part of the STN. 


\section{Introduction}

The STN's involvement in limbic functions can be explained by its connections to cortical and subcortical limbic regions via the cortico-basal ganglia-thalamocortical circuits (Alexander, et al., 1990, Alexander, et al., 1986, Tan, et al., 2011). The STN is generally subdivided into the dorsolateral (somatomotor), the ventromedial (associative), and the medial tip (limbic) parts (Temel, et al., 2005). The medial (ventromedial and medial tip) part of the STN is reciprocally connected with the ventral parts of the globus pallidus, which receives projections from the ventromedial prefrontal cortex (vmPFC), cingulate gyrus (Cg), and the medial part of the parafascicular thalamic nucleus, and is indirectly connected with the nucleus accumbens (NAc) (Berendse and Groenewegen, 1991, Janssen, et al., 2010, Kolomiets, et al., 2001). Interestingly, stimulation of the NAc, vmPFC and subgenual $\mathrm{Cg}$ has been shown to induce antidepressant-like responses in both patients (Kennedy, et al., 2011, Schlaepfer, et al., 2008) and animal models (Falowski, et al., 2011, Hamani and Nobrega, 2012). The relationship between the antidepressant-like effects induced by stimulation of these areas and the medial part of the STN remains largely unknown. Here, we addressed the research question whether the medial part of the STN is involved in the mood-related effects of stimulation of the NAC, vmPFC, and Cg. Utilising animal models, we performed HFS of the NAc, vmPFC, and Cg and tested for hedonia and forced swim immobility. Subsequently, we measured the effect of HFS on changes in neuronal activity of the subdivisions of the STN using c-Fos immunoreactivity (c-Fos-ir).

\section{Material and Methods}

\section{Subjects}

Sixteen week-old male Sprague-Dawley rats (Harlan, Horst, the Netherlands; $\mathrm{n}=30$ ) were housed at the Central Animal Facility of Maastricht University (Maastricht, the Netherlands) with body weight of 320-370 g at the time of surgery. Electrodes were implanted in the NAc, VmPFC, and $\mathrm{Cg}$. Rats were randomly assigned to one of the following experimental groups: 1. HFS NAc $(n=6) ; 2$. HFS vmPFC $(n=6) ; 3$. HFS Cg $(n=6)$; and 4. Sham $(n=12)$.

\section{Surgery}

Details of the surgical procedure and the electrodes used have been described earlier (Lim, et al., 2008, Tan, et al., 2010). In brief, 30 min before surgery rats were injected with Temgesic $(0.1 \mathrm{mg} / \mathrm{kg}$, s.c.). Throughout surgery, rats were anesthetized by $2 \%$ isoflurane inhalation (IsoFlo ${ }^{\circledR}$, Abbott Laboratories Ltd, Berkshire, UK). The rat was placed in a stereotactic apparatus (Stoelting, Wood Dale, USA;model 51653) two burr 
holes were drilled and a bilateral electrode was implanted according to the following Bregma coordinates (mm): vmPFC: AP+2.7, ML 0.6, DV -4.6; Cg: AP +1.6, ML 0.6, DV -2.8 mm; NAc: AP +2.2 mm, ML $1.5 \mathrm{~mm}$, DV -6.8 mm) (Paxinos and Watson, 1998). Electrodes were fixed to the skull with screws and dental cement (Heraues Kulzer, Hanau, Germany). After surgery, rats were injected with Temgesic (buprenorfine hydrochloride, $0.1 \mathrm{mg} / \mathrm{kg}$, s.c.) for analgesia and received a period of two-week recovery.

\section{Stimulation}

Two weeks after surgery rats were stimulated for behavioural tests with monophasic pulses with an amplitude of $100 \mu \mathrm{A}$ and a pulse width of $100 \mu \mathrm{s}$ at $100 \mathrm{~Hz}$ (HFS), using an isolator (DLS100, WPI, Berlin, Germany) that was connected to a digital stimulator (DS8000, WPI, Berlin, Germany). In the experimental groups, HFS was applied based on previous experimental studies showing the most effective stimulation parameter for antidepressant response (Hamani, et al., 2010, Hamani, et al., 2010). The sham animals were implanted with electrodes and similarly treated as the HFS animals, but without stimulation.

\section{Behavioural Testing}

\section{Sucrose Intake Test}

The sucrose intake test was performed over a period of two days. A day prior to the test, animals were trained to drink $1 \%$ sucrose solution by exposing them to sucrose instead of water during $1 \mathrm{~h}$. After a period of $14 \mathrm{~h}$ of food and water deprivation, animals were offered sucrose for $1 \mathrm{~h}$ and tested for the stimulation effect. The consumption of the sucrose solution was measured simultaneously by weighing the bottles before and after the test. The sucrose intake was calculated based on the total amount of sucrose solution consumed in each animal.

\section{Forced Swim Test}

The FST was carried out using a transparent Perspex cylinder (50 x $20 \mathrm{~cm})$ separated by black side walls and placed on a black base $60 \mathrm{~cm}$ from the floor. The cylinder was filled with tap water $\left(25 \pm 1^{\circ} \mathrm{C}\right)$ to a depth of $30 \mathrm{~cm}$ (Cryan, et al., 2005, Temel, et al., 2007). Testing was carried out over two consecutive days. In the pre-test session, each rat was placed in the water for $15 \mathrm{~min}$. The following day, rats were placed in the water for 10 min. Electrical stimulation commenced 2 min before testing and continued during the entire duration of testing. Recordings of behaviour were taken by a digital camera. The duration of the following behaviours was measured by observer blind to treatments: "immobility" (no movements or small and infrequent movements performed solely to 
maintain the nose above the water), "swimming" (active swimming with the forepaws), and "climbing" (scratching of cylinder walls using both forepaws and hindpaws).

\section{Histology and immunohistochemistry}

90 min after the final 30 min of electrical stimulation procedure, rats were placed under generalized anaesthesia with Nembutal $(75 \mathrm{mg} / \mathrm{kg}$ ) and perfused transcardially with Tyrode $(0,1 \mathrm{M})$ and fixative containing $15 \%$ picric acid, $4 \%$ paraformaldehyde, and $0,05 \%$ glutaraldehyde in 0,1 M phosphate buffer $(\mathrm{pH}$ 7.6). Brains were removed, postfixed for 2 hours, and cryoprotected overnight in 15\% sucrose in 0,1 M phosphate buffer ( $\mathrm{pH}$ 7.6). Brain tissue was then quickly frozen with $\mathrm{CO}_{2}$ and stored at $-80{ }^{\circ} \mathrm{C}$. Subsequently, the brains were cut serially (10 series) on a cryostat (MICROM, Germany) into $30 \mu \mathrm{m}$ frontal sections and again stored at $-80^{\circ} \mathrm{C}$.

\section{c-Fos Immunohistochemistry}

C-Fos immunoreactivity (c-Fos-ir) stainings were carried out using a primary antibody polyclonal rabbit anti-c-Fos in 0.1\% Bovine Serum Albumin and Tris Buffered SolutionTriton (TBS-T) solution (diluted 1: 20.000, Santa Cruz Biotechnology Inc,. Santa Cruz, CA, USA). Details of this staining have been previously described (Lim, et al., 2008, Lim, et al., 2009). Sections were incubated overnight at room temperature on a constant shaker and rinsed with TBS-T, Tris Buffered Solution (TBS), and TBS-T. After rinsing the sections were incubated with the secondary donkey anti-rabbit antibody in biotinylated biotine (diluted 1:400; Jackson Immunoresearch Laboratories Inc., Westgrove, USA) for 90 min. After washing with TBS and TBS-T the sections were incubated with an avidinbiotin-peroxidase complex (diluted 1:800, Elite ABC-kit, Vectastatin; Burlingname, USA) for $2 \mathrm{~h}$. To visualize the immune complex of Horseradish Peroxide (HRP) reaction product, sections were incubated with 3,3'-diaminobenzidine tetrahydrochloride (DAB)/ Nickel Chloride $\left(\mathrm{NiCL}_{2}\right.$ ) solution (5 ml DAB solution, $5 \mathrm{ml} \mathrm{Tris/} \mathrm{HCl}, 50 \mu \mathrm{NiCL}$, and 3.35 $\mu l$ hydrogen peroxide). After $10 \mathrm{~min}$ this reaction was stopped by rinsing thoroughly all the sections with TBS. All the sections were then mounted on gelatine-coated glasses. After dehydrating, all sections were cover-slipped with Pertex (HistolabProducts ab, Goteborg, Sweden).

Systematic quantitative cell counts of c-Fos-ir cells were performed in the STN. Photographs of the STN were taken at 4x magnification using an Olympus DP70 camera connected to an Olympus AX70 bright-field microscope (analysis; Imaging System, Münster, Germany). The same light intensity and threshold conditions were employed for all sections. The boundaries of the STN were delineated and measured according to the Paxinos and Watson rat brain atlas (Paxinos and Watson, 1998). Using the conventional image analysis program 'Image J' (version 1.38, NIH, USA) the counting of the numbers of c-Fos-ir cells was performed. A cell was counted as c-Fos-ir positive if the optical 
density was $50 \%$ higher than the mean background density of that section. The STN was divided in two parts to distinguish between c-Fos activation in the medial and lateral part of the nucleus (see Fig. 1). The number of c-Fos-ir cells per $\mathrm{mm}^{2}$ was calculated for each area of interest.

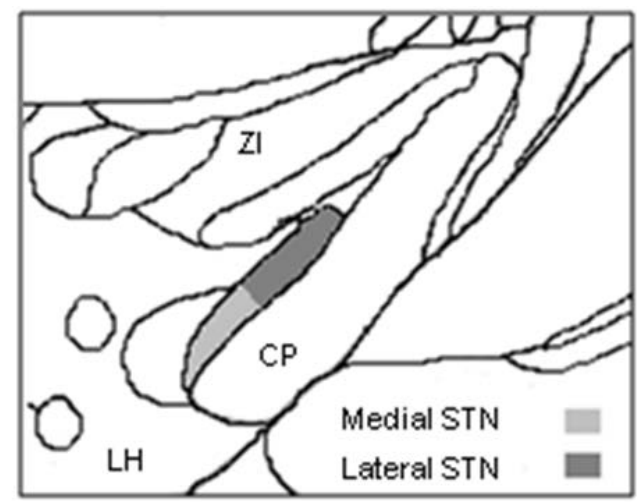

Fig. 1. The diagram shows how the STN was divided into two parts as medial and lateral STN for counting the number of c-Fos-ir cells per $\mathrm{mm}^{2}$. The light and dark grey represent respectively the medial and lateral part of the STN. Abbreviations: CP cerebral peduncle, LH: lateral hypothalamus, ZI: zona incerta.

\section{Electrode localization}

Coronal sections containing the electrode trajectory were mounted on gelatin-coated slides, and stained with a standard hematoxylin-eosin (Merck, Darmstadt, Germany) histological procedure to examine the localization of the electrode tips and its surrounding morphological changes due to the stimulation or implantation effects.

\section{Data and statistical analysis}

All data were analyzed using one-way ANOVA followed by an LSD post-hoc test to analyze group differences in more detail. Analysis was performed with SPSS statistical software (SPSS version 15.0, IBM, Chicago, USA). All data are presented as means and standard errors of means (SEM). $\mathrm{p}<0.05$ was considered to be statistically significant.

\section{Results}

Electrode tips were located within the region of their respective coordinates. The localizations of the electrode tips for HFS NAc, HFS vmPFC, and HFS Cg are illustrated in Fig. 2. Animals with misplacement of electrodes were discarded from analysis. No histological damage due HFS was observed. 
(A)

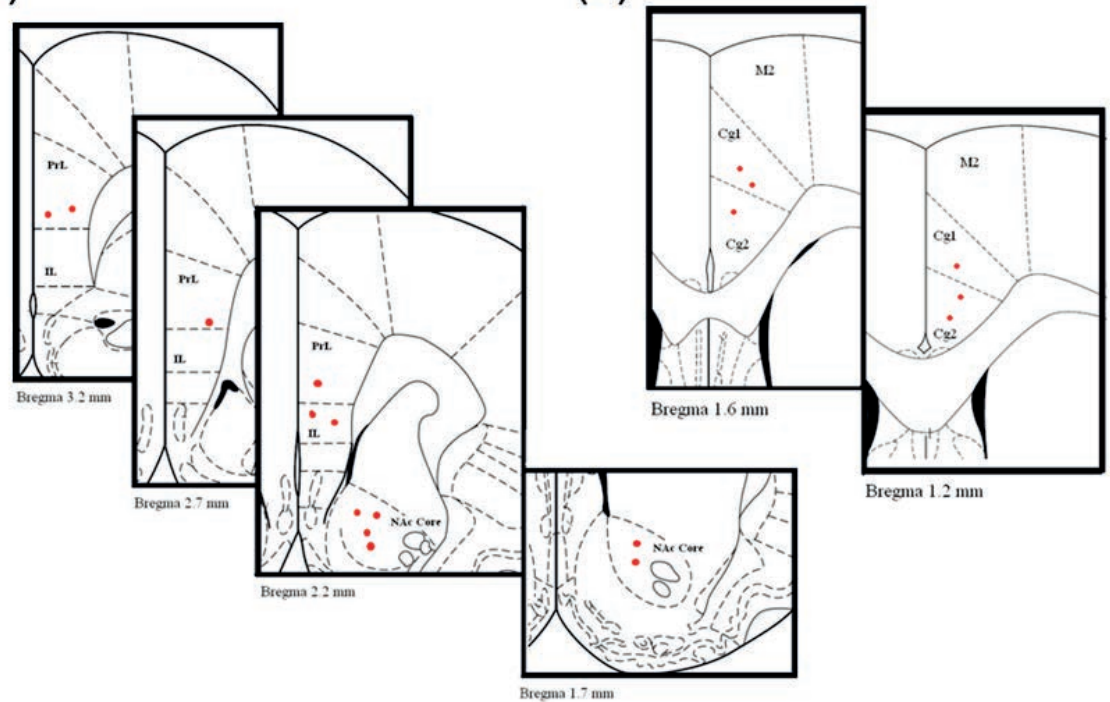

Fig. 2. Schematic representation of the electrode sites in the NAc and vmPFC (Fig. 2A), and Cg (Fig. 2B) according to the Paxinos and Watson atlas (Paxinos and Watson, 1998), respectively. The symbol $(\bullet)$ indicates the tips of electrode localization. Abbreviations: Cg: Cingulate Gyrus, IL: Infralimbic, PrL: Prelimbic, M1: Primary Motor Cortex, M2: Secondary Motor Cortex, and NAc Core: Nucleus Accumbens Core.

HFS of the NAc and VMPFC, but not Cg significantly increased the level of sucrose consumption $\left(F_{(3,20)}=5.407, p=0.007\right.$, fig 3$)$, and reduced forced swim immobility $\left(F_{(3,19)}=6.610, p=0.003\right)$ as compared to sham animals (Fig. 4). Despite no significant difference was found in the swimming time $\left(F_{(3,18)}=1.339, p=n . s\right.$. $)$, there was a remarkable increase of climbing time $\left(F_{(3,20)}=5.521, p=0.006\right)$ with NAc HFS in the forced swim test.

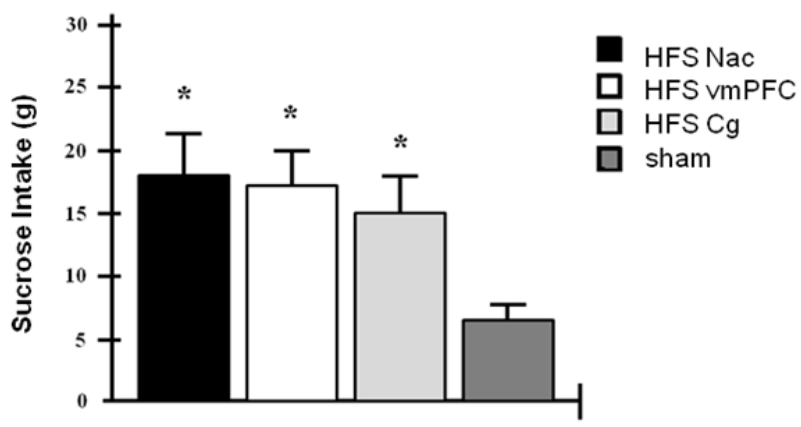

Fig. 3. A set of bar graphs showing the total level of sucrose consumption after high-frequency stimulation of naïve rats during $1 \mathrm{~h}$ of testing. Note, significant increase of consumption was observed with stimulation of the NAc, vmPFC, the Cg regions Data represent means \pm S.E.M. Indication: ${ }^{*}$, significant difference from the sham animals, $(P<0.05)$. Abbreviations: $C g$ : Cingulate Gyrus, DBS: deep brain stimulation, NAc: Nucleus Accumbens, vmPFC: ventromedial prefrontal cortex. 

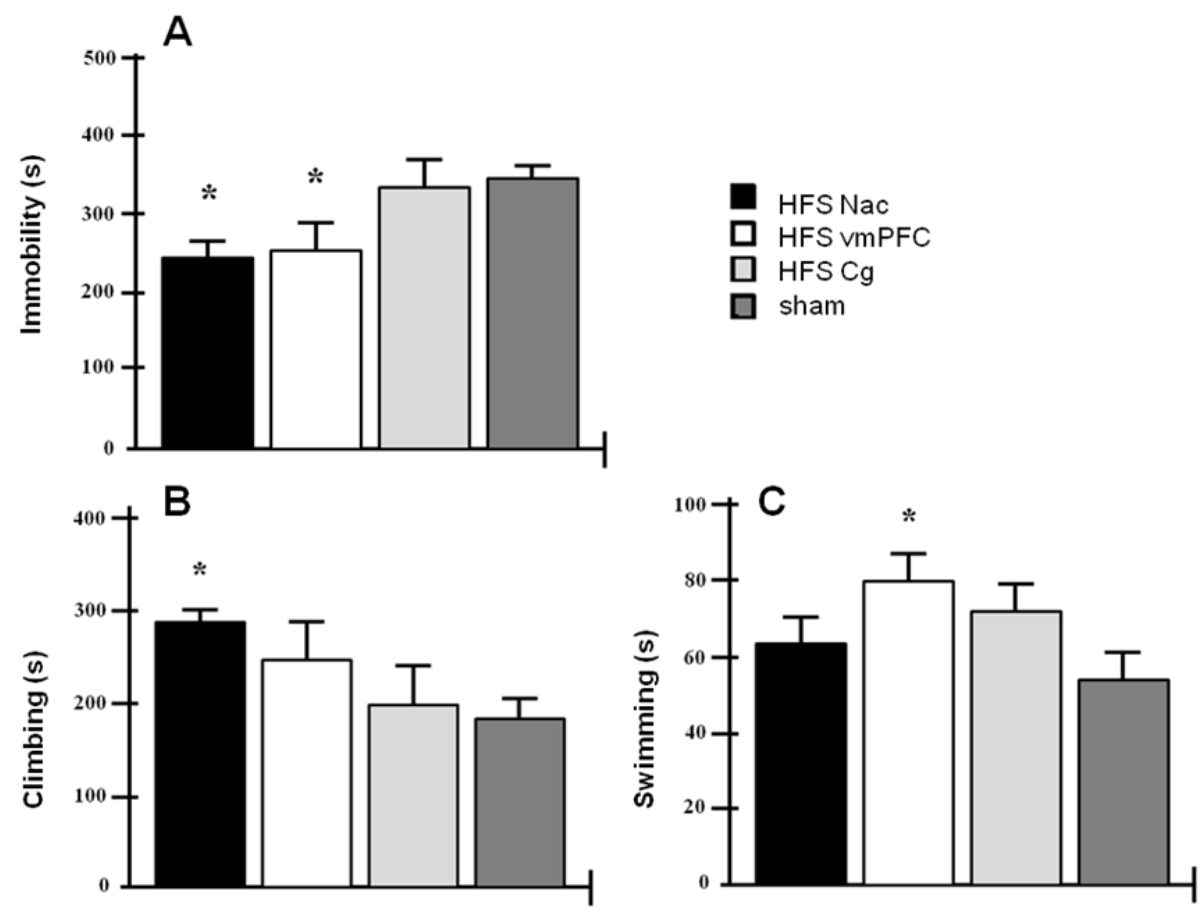

Fig. 4. A set of bar graphs showing the different behavioural measures after high-frequency stimulation of naïve rats in the forced swim test. Note, stimulation of the NAc and vmPFC significantly reduced behavioural despair measure. Data represent means \pm S.E.M. Indication: ${ }^{*}$, significant difference from the sham animals, $(P<0.05)$. Abbreviations: $C g$ : Cingulate Gyrus, DBS: deep brain stimulation, NAc: Nucleus Accumbens, vmPFC: ventromedial prefrontal cortex.

Since no significant difference ( $p$ 's $>0.05$ ) was found in the number of c-Fos-ir cells in the ipsilateral and contralateral regions of the medial and lateral STN, the data were pooled. Bonferroni post hoc test revealed that HFS of the VmPFC significantly increased the number of $c$-Fos-ir cells $\left(F_{(3,17)}=6.850, p=0.003\right)$ in the medial STN compared to nonstimulated sham animals (Fig. 5). No significant difference was found in animals with HFS of the NAc and $\mathrm{Cg}$. In addition, the number of c-Fos-ir cells was also higher in the medial STN of animals received NAC and vmPFC HFS when compared to the lateral STN (all $t_{(4)}=3.381, p<0.028$; Fig. 5), respectively. No significant group difference was observed in the lateral STN $\left(F_{(3,17)}=1.595, p=n . s.\right)$.

Since vmPFC HFS significantly enhanced c-Fos-ir in the medial STN, we next addressed the question whether the behavioral effects were associated with the c-Fos-ir of the medial STN. Our results show that the forced swim immobility and sucrose intake level were strongly correlated with the c-Fos-ir in the medial STN of animals with vmPFC HFS (Fig. 6A, B). No correlation was observed between the activity changes in the lateral STN and the behavioural parameters, as well as in the sham-treated animals (Fig. 6C). 


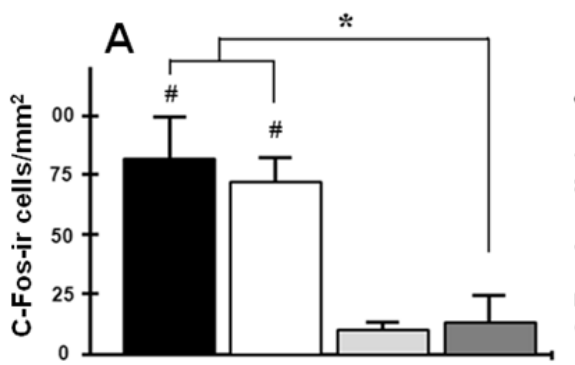

Medial STN

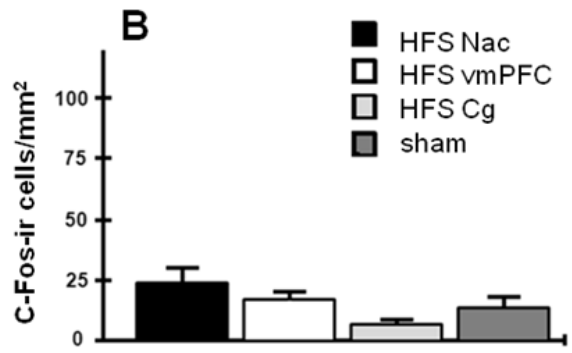

LateralSTN

Fig. 5. A set of bar graphs showing the effects of stimulation on the number of c-Fos-ir cells per $\mathrm{mm}^{2}$ in the medial and lateral part of the STN. Note: HFS of the VmPFC and NAc remarkably increased the c-Fos-ir expression in the medial part of the STN in comparison to sham. Data represent means \pm S.E.M. Indication: *, significant difference from the sham animals, $(P<0.05)$; \#, significant different to the lateral STN of respective group. Abbreviations: Cg: Cingulate Gyrus, DBS: deep brain stimulation, NAc: Nucleus Accumbens, vmPFC: ventromedial prefrontal cortex.

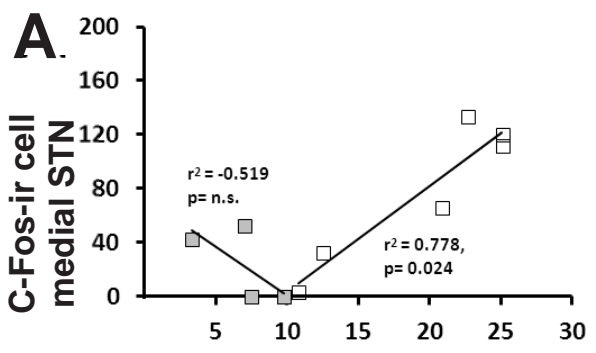

Sucrose Intake

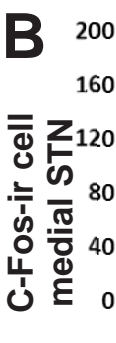

$200 \quad 300 \quad 400 \quad 500$

Forced Swim

\begin{tabular}{|c|c|c|c|c|c|c|}
\hline & Medial STN & Lateral STN & Climbing & Swimming & Immobility & $\begin{array}{c}\text { Sucrose } \\
\text { Intake }\end{array}$ \\
\hline Medial STN & & .775 & .569 & .380 & $-.926^{*}$ & $.936^{*}$ \\
\hline Lateral S TN & .827 & & -.030 & 662 & -.719 & .512 \\
\hline Climbing & -.452 & -.227 & & -.432 & -.539 & 817 \\
\hline Swimming & .067 & .204 & -.266 & & -.468 & .101 \\
\hline Immobility & .340 & .038 & -661 & -.548 & & -.868 \\
\hline Sucrose Intake & -.721 & -.604 & .792 & -.624 & -.202 & \\
\hline
\end{tabular}

Fig. 6. Scatter plots (A, B) and table (C) display the correlations between the behavioral variables and the STN c-Fos-ir. Note. The medial STN is strongly correlated with the depressive-like behaviors in animals of vmPFC HFS. No correlations were observed in the lateral STN, and the sham animals. Data represent means \pm S.E.M. Indication: ${ }^{*}$, significant difference from the sham animals, $(p<0.05)$; \#, significant different from the lateral STN of respective group. Abbreviations: STN: subthalamic nucleus, vmPFC: ventromedial prefrontal cortex. 


\section{Discussion}

In the present study, we found that HFS of the NAC and VmPFC, but not $\mathrm{Cg}$ reduced forced swim immobility and anhedonic-like behavior in the sucrose intake test, indicating antidepressant-like response (Cryan, et al., 2002, Forbes, et al., 1996). In addition, HFS of the VMPFC, but not NAc and Cg, enhanced c-Fos-ir significantly and specifically in the medial part of the STN. To determine the neural-behavioral correlates of stimulation effects, a significant correlation was observed between the medial STN c-Fos-ir and depressive-anhedonic-like behaviors (forced swim immobility and sucrose intake) in animals with vmPFC HFS. Overall, our data showed no correlation between the cellular activity in the lateral STN and parameters of the forced swim and sucrose intake tests.

The current findings of HFS of the NAc and VmPFC reproduced the antidepressantlike effects of previous clinical (Mayberg, et al., 2005, Schlaepfer, et al., 2008) and animal studies (Falowski, et al., 2011, Hamani, et al., 2010, Hamani, et al., 2010). A possible explanation that HFS of these two targets was effective in treating depressive-like behavior can be partially explained in terms of their stimulation-induced neurochemical effects in the forebrain. HFS of the NAc has been shown to increase the levels of tyrosine hydroxylase (Falowski, et al., 2011), and serotonin (5-hydroxytryptamine, 5-HT) levels in the MPFC with approximately $27 \%$ increase as compared to the baseline (van Dijk, et al., 2012). Interestingly, vmPFC HFS also enhanced the 5-HT levels with remarkable fourfold increase in the hippocampus by fourfold (Hamani, et al., 2010, Hamani, et al., 2011). On the other hand, we showed no antidepressant effects with HFS of the Cg. This is in line with a previous study which demonstrated that stimulation of certain cortical areas did not affect the 5-HT output in either hippocampus or amygdala (Juckel, et al., 1999). Based on this evidence, it is suggested that the antidepressant effects of NAc and vmPFC HFS on 5-HT neurotransmission were likely to be associated with their projections to the midbrain dorsal raphe nuclei (Peyron, et al., 1998). In contrast, STN stimulation, which can evoke depressive-like behavior, is known to reduce central levels of 5-HT by a dorsal raphe nucleus dependent mechanism (Navailles, et al., 2010, Tan, et al., 2012, Temel, et al., 2007). Taken together, these studies indicate that the regulation of depressive-like behavior by stimulation of these areas was probably mediated by a mechanism of 5-HT dependant neurotransmission.

The increase of neuronal c-Fos-ir in the medial STN by vmPFC HFS is in line with an earlier study which showed a neuronal activation in the medial STN following stimulation of the prelimbic cortical regions (Sgambato, et al., 1997). However, the authors also found an enhanced neuronal activation in the lateral STN following stimulation of the motor cortex. One possible explanation for this observation could probably due to the direct projection of excitatory glutamatergic input from the VmPFC to the medial STN (Nambu, et al., 2002) via a monosynaptic connection between the cortex and STN. In 
anatomical tracing studies, evidence clearly showed that the anterogradely-labelled fibers, originating from the MPFC, were found in the medial STN (Berendse and Groenewegen, 1991, Kolomiets, et al., 2001). Besides, data from electrophysiological study has also demonstrated that MPFC stimulation induced excitatory neuronal responses which was specifically located in the medial part of the STN, whereas cells that did not respond were located more laterally (Maurice, et al., 1998). Further, the medial STN also receives an indirect excitatory input from the vmPFC through a NAc-ventral pallidum-STN disinhibitory circuit. The STN is known to project to the MPFC directly via a subthalamic-cortical projection (Degos, et al., 2008), but also indirectly through basal ganglia-thalamocortical connections (Temel, et al., 2005). At the behavioural level, it is important to highlight that the disconnection of VMPFC-STN showed impairment on attention and emotional behavior deficits (Chudasama, et al., 2003). Overall, these studies demonstrate that different parts of the STN receive specific cortical projections from the motor/premotor, and the limbic areas, particularly the vmPFC for control of limbic behaviors (Chudasama, et al., 2003, Maurice, et al., 1999).

In the present study, our correlation data have revealed a significant association between the medial STN cellular c-Fos-ir and the HFS-induced antidepressant-like behaviors. Although the medial STN is generally regarded as an integrator and processes limbic and associative information, our results strongly suggest that the medial STN has specific functional roles for depressive-like behaviors that depend largely on which limbic projection pathway was stimulated.

In conclusion, HFS of the NAc and vmPFC produced remarkable antidepressant-like responses with significant increased sucrose intake and reduction of forced swim immobility behaviors. We found that vmPFC HFS enhanced the cellular activity within the medial part of the STN. This increase was correlated with the antidepressant-like behaviors in animals with vmPFC HFS.

\section{Acknowledgements}

This research was supported by grants from the Netherlands Organization for Scientific Research (NWO Veni, No: 016.096.032), and the Singapore Lee Kuan Yew Research Fellowship (M4080846.080). 


\section{References}

1. Baunez, C., Christakou, A., Chudasama, Y., Forni, C., and Robbins, T. W., 2007. Bilateral high-frequency stimulation of the subthalamic nucleus on attentional performance: transient deleterious effects and enhanced motivation in both intact and parkinsonian rats. Eur J Neurosci 25, 1187-1194.

2. Baunez, C., Nieoullon, A., and Amalric, M., 1995. In a rat model of parkinsonism, lesions of the subthalamic nucleus reverse increases of reaction time but induce a dramatic premature responding deficit. J Neurosci 15, 6531-6541.

3. Berendse, H. W., and Groenewegen, H. J., 1991. The connections of the medial part of the subthalamic nucleus in the rat: evidence for a parallel organization. The basal ganglia III. New York: Plenum., 89-98.

4. Canteras, N. S., Shammah-Lagnado, S. J., Silva, B. A., and Ricardo, J. A., 1990. Afferent connections of the subthalamic nucleus: a combined retrograde and anterograde horseradish peroxidase study in the rat. Brain Res 513, 43-59.

5. Carpenter, M. B., Whittier, J. R., and Mettler, F. A., 1950. Analysis of choreoid hyperkinesia in the Rhesus monkey; surgical and pharmacological analysis of hyperkinesia resulting from lesions in the subthalamic nucleus of Luys. J Comp Neurol 92, 293-331.

6. Crossman, A. R., 1987. Primate models of dyskinesia: the experimental approach to the study of basal ganglia-related involuntary movement disorders. Neuroscience 21, 1-40.

7. Cryan, J. F., Markou, A., and Lucki, I., 2002. Assessing antidepressant activity in rodents: recent developments and future needs. Trends Pharmacol Sci 23, 238-245.

8. Cryan, J. F., Valentino, R. J., and Lucki, I., 2005. Assessing substrates underlying the behavioral effects of antidepressants using the modified rat forced swimming test. Neurosci Biobehav Rev 29, 547-569.

9. Degos, B., Deniau, J. M., Le Cam, J., Mailly, P., and Maurice, N., 2008. Evidence for a direct subthalamocortical loop circuit in the rat. Eur J Neurosci 27, 2599-2610.

10. Falowski, S. M., Sharan, A., Reyes, B. A., Sikkema, C., Szot, P., and Van Bockstaele, E. J., 2011. An evaluation of neuroplasticity and behavior after deep brain stimulation of the nucleus accumbens in an animal model of depression. Neurosurgery 69, 1281-1290.

11. Fawcett, J., Clark, D. C., Scheftner, W. A., and Gibbons, R. D., 1983. Assessing anhedonia in psychiatric patients. Arch Gen Psychiatry 40, 79-84.

12. Forbes, N. F., Stewart, C. A., Matthews, K., and Reid, I. C., 1996. Chronic mild stress and sucrose consumption: validity as a model of depression. Physiol Behav 60, 1481-1484.

13. Groenewegen, H. J., and Berendse, H. W., 1990. Connections of the subthalamic nucleus with ventral striatopallidal parts of the basal ganglia in the rat. J Comp Neurol 294, 607-622.

14. Groenewegen, H. J., Berendse, H. W., and Haber, S. N., 1993. Organization of the output of the ventral striatopallidal system in the rat: ventral pallidal efferents. Neuroscience 57, 113-142.

15. Hamani, C., Diwan, M., Isabella, S., Lozano, A. M., and Nobrega, J. N., 2010. Effects of different stimulation parameters on the antidepressant-like response of medial prefrontal cortex deep brain stimulation in rats. J Psychiatr Res 44, 683-687.

16. Hamani, C., Diwan, M., Macedo, C. E., Brandao, M. L., Shumake, J., Gonzalez-Lima, F., Raymond, R., Lozano, A. M., Fletcher, P. J., and Nobrega, J. N., 2010. Antidepressant-like effects of medial prefrontal cortex deep brain stimulation in rats. Biol Psychiatry 67, 117-124.

17. Hamani, C., Machado, D. C., Hipolide, D. C., Dubiela, F. P., Suchecki, D., Macedo, C. E., Tescarollo, F., Martins, U., Covolan, L., and Nobrega, J. N., 2011. Deep Brain Stimulation Reverses Anhedonic-Like Behavior in a Chronic Model of Depression: Role of Serotonin and BDNF. Biol Psychiatry in press.

18. Janssen, M., Visser-Vandewalle, V., and Temel, Y., 2010. Cortico-subthalamic projections in the rat. J Exp Clin Med 27, 4-12.

19. Kolomiets, B. P., Deniau, J. M., Mailly, P., Menetrey, A., Glowinski, J., and Thierry, A. M., 2001. Segregation and convergence of information flow through the cortico-subthalamic pathways. J Neurosci $21,5764-5772$. 
20. Krack, P., Batir, A., Van Blercom, N., Chabardes, S., Fraix, V., Ardouin, C., Koudsie, A., Limousin, P. D., Benazzouz, A., LeBas, J. F., Benabid, A. L., and Pollak, P., 2003. Five-year follow-up of bilateral stimulation of the subthalamic nucleus in advanced Parkinson's disease. N Engl J Med 349, 1925-1934.

21. Leichnetz, G. R., Hardy, S. G., and Carruth, M. K., 1987. Frontal projections to the region of the oculomotor complex in the rat: a retrograde and anterograde HRP study. J Comp Neurol 263, 387-399.

22. Lim, L. W., Blokland, A., Visser-Vandewalle, V., Vlamings, R., Sesia, T., Steinbusch, H., Schruers, K., Griez, E., and Temel, Y., 2008. High-frequency stimulation of the dorsolateral periaqueductal gray and ventromedial hypothalamus fails to inhibit panic-like behaviour. Behav Brain Res 193, 197-203.

23. Lim, L. W., Temel, Y., Visser-Vandewalle, V., Blokland, A., and Steinbusch, H., 2009. Fos immunoreactivity in the rat forebrain induced by electrical stimulation of the dorsolateral periaqueductal gray matter. J Chem Neuroanat 38, 83-96.

24. Limousin, P., Pollak, P., Benazzouz, A., Hoffmann, D., Broussolle, E., Perret, J. E., and Benabid, A. L., 1995. Bilateral subthalamic nucleus stimulation for severe Parkinson's disease. Mov Disord 10, 672-674.

25. Mallet, L., Schupbach, M., N'Diaye, K., Remy, P., Bardinet, E., Czernecki, V., Welter, M. L., Pelissolo, A., Ruberg, M., Agid, Y., and Yelnik, J., 2007. Stimulation of subterritories of the subthalamic nucleus reveals its role in the integration of the emotional and motor aspects of behavior. Proc Natl Acad Sci U S A 104, 10661-10666.

26. Martin, J. P., 1927. Hemichorea resulting from a focal lesion of he brain (The syndrome of the body of Luys). Brain, 637.

27. Maurice, N., Deniau, J. M., Glowinski, J., and Thierry, A. M., 1998. Relationships between the prefrontal cortex and the basal ganglia in the rat: physiology of the corticosubthalamic circuits. J Neurosci 18, 95399546.

28. Mayberg, H. S., Lozano, A. M., Voon, V., McNeely, H. E., Seminowicz, D., Hamani, C., Schwalb, J. M., and Kennedy, S. H., 2005. Deep brain stimulation for treatment-resistant depression. Neuron 45, 651-660.

29. Miller, W. C., and DeLong, M. R., 1987. Altered tonic activity of neurons in the globus pallidus and subthalamic nucleus in the primate model of parkinsonism. In: Carpenter, M. B., and Jayaraman, A., (Eds.), The Basal Ganglia II: structure and function. Plenum, New York, pp. 415-427.

30. Nagy, J. I., Carter, D. A., Lehmann, J., and Fibiger, H. C., 1978. Evidence for a GABA-containing projection from the entopeduncular nucleus to the lateral habenula in the rat. Brain Res 145, 360-364.

31. Navailles, S., Benazzouz, A., Bioulac, B., Gross, C., and De Deurwaerdere, P., 2010. High-frequency stimulation of the subthalamic nucleus and L-3,4-dihydroxyphenylalanine inhibit in vivo serotonin release in the prefrontal cortex and hippocampus in a rat model of Parkinson's disease. J Neurosci 30, 2356-2364.

32. Orieux, G., Francois, C., Feger, J., and Hirsch, E. C., 2002. Consequences of dopaminergic denervation on the metabolic activity of the cortical neurons projecting to the subthalamic nucleus in the rat. J Neurosci 22, 8762-8770.

33. Paxinos, G., and Watson, C., 1998. The Rat Brain in Stereotaxic Coordinates. Academic Press, New York.

34. Schlaepfer, T. E., Cohen, M. X., Frick, C., Kosel, M., Brodesser, D., Axmacher, N., Joe, A. Y., Kreft, M., Lenartz, D., and Sturm, V., 2008. Deep brain stimulation to reward circuitry alleviates anhedonia in refractory major depression. Neuropsychopharmacology : official publication of the American College of Neuropsychopharmacology 33, 368-377.

35. Sgambato, V., Abo, V., Rogard, M., Besson, M. J., and Deniau, J. M., 1997. Effect of electrical stimulation of the cerebral cortex on the expression of the Fos protein in the basal ganglia. Neuroscience 81, 93-112.

36. Tan, S., Vlamings, R., Lim, L., Sesia, T., Janssen, M. L., Steinbusch, H. W., Visser-Vandewalle, V., and Temel, Y., 2010. Experimental deep brain stimulation in animal models. Neurosurgery 67, 1073-1079; discussion1080.

37. Tan, S. K., Hartung, H., Visser-Vandewalle, V., Steinbusch, H. W., Temel, Y., and Sharp, T., 2012. A combined in vivo neurochemical and electrophysiological analysis of the effect of high-frequency stimulation of the subthalamic nucleus on 5-HT transmission. Exp Neurol 233, 145-153. 
38. Tan, S. K., Janssen, M. L., Jahanshahi, A., Chouliaras, L., Visser-Vandewalle, V., Lim, L. W., Steinbusch, H. W., Sharp, T., and Temel, Y., 2011. High frequency stimulation of the subthalamic nucleus increases c-fos immunoreactivity in the dorsal raphe nucleus and afferent brain regions. J Psychiatr Res 45, 1307-1315.

39. Temel, Y., Blokland, A., Steinbusch, H. W., and Visser-Vandewalle, V., 2005. The functional role of the subthalamic nucleus in cognitive and limbic circuits. Prog Neurobiol 76, 393-413.

40. Temel, Y., Boothman, L. J., Blokland, A., Magill, P. J., Steinbusch, H. W., Visser-Vandewalle, V., and Sharp, T., 2007. Inhibition of 5-HT neuron activity and induction of depressive-like behavior by high-frequency stimulation of the subthalamic nucleus. Proc Natl Acad Sci U S A 104, 17087-17092.

41. Temel, Y., Kessels, A., Tan, S., Topdag, A., Boon, P., and Visser-Vandewalle, V., 2006. Behavioural changes after bilateral subthalamic stimulation in advanced Parkinson disease: a systematic review. Parkinsonism Relat Disord 12, 265-272.

42. Visser-Vandewalle, V., van der Linden, C., Temel, Y., Celik, H., Ackermans, L., Spincemaille, G., and Caemaert, J., 2005. Long-term effects of bilateral subthalamic nucleus stimulation in advanced Parkinson disease: a four year follow-up study. Parkinsonism Relat Disord 11, 157-165.

43. Whittier, J. R., and Mettler, F. A., 1949. Studies on the subthalamus of the rhesus monkey; hyperkinesia and other physiologic effects of subthalamic lesions; with special reference to the subthalamic nucleus of Luys. J Comp Neurol 90, 319-372. 


\section{CHAPTER 5}

Functional cortico-subthalamic inputs from the motor, limbic and associative areas in normal and dopamine depleted rats

Janssen MLF, Temel Y, Delaville C, Zwartjes DGM,

Heida T, Visser-Vandewalle V, Benazzouz A

Under preparation for submission 


\section{Abstract}

Introduction: The key role of the STN in the basal ganglia is reflected by its therapeutic role for Parkinson's disease in which it is a target for deep brain stimulation (DBS). Two major concepts of cortico-basal ganglia information flow exist: one supporting parallel information flow from functionally different cortical regions through the basal ganglia and the other supporting integration of information. It remains unclear whether these different circuits are segregated or integrated within the STN. To address this question, we performed an electrophysiological study in the rat and investigated the responses of individual STN cells to the electrical stimulation of different cortical regions in control and dopamine depleted rodents.

Method: Motor (MC), cingulate (CG), pre-limbic (PrL), infra-limbic (IL) and agranular insular (AI) cortices were stimulated. Peristimulus time histograms (PSTHs) were generated and responses were analysed. All neurons were anatomically mapped, based on their cortical input.

Results: In control and sham animals, responses to $\mathrm{MC}, \mathrm{CG}, \mathrm{PrL}, \mathrm{IL}$ and $\mathrm{Al}$ were seen. 6-OHDA lesioned animals showed the same kind of responses as control animals. Most neurons responded to only one cortical region, but in both control and 6-OHDA animals about $30 \%$ of the neurons showed an early response to both $\mathrm{MC}$ and $\mathrm{Al}$, even more neurons responded with a late excitation to stimulation of different cortical regions. Neuronal responses to cortical stimulation of the STN were grossly anatomically organized with large overlap and interaction between different functional corticosubthalamic pathways is seen electrophysiologically.

Conclusion: Subthalamic neurons a grossly functionally organized with overlap between them. Also a large number of neurons showed responses from different cortical regions, which provides evidence for the integration of motor and non-motor cortico-subthalamic pathways. 


\section{Introduction}

The subthalamic nucleus (STN) possesses a central position in the basal ganglia circuitry (Albin et al., 1989, Smith et al., 1998). Classically, the STN receives its input from the globus pallidus externus (GPe), as a part of the indirect pathway. In addition a high density of cortical terminals is present in the STN; this monosynaptic, cortico-STN projection is also known as the 'hyperdirect' pathway (Nambu et al., 2002). As a driving force of the basal ganglia, the STN strongly controls the basal ganglia output nuclei by its excitatory glutamatergic efferents. Moreover the STN is also an output nucleus itself by its efferent projections to the cortex (Miyachi et al., 2006, Degos et al., 2008). The key role of the STN in the basal ganglia motor and non-motor information processing is nicely reflected by its therapeutic role for Parkinson's disease (PD) in which it is a target for deep brain stimulation (DBS). In MPTP monkeys (Benazzouz et al., 1993, Benazzouz et al., 1996) and later in PD patients, STN DBS alleviates motor symptoms (Benazzouz et al., 1993, Limousin et al., 1995, Benazzouz et al., 1996, Krack et al., 2003, Janssen et al., 2014).

Currently, two major concepts of cortico-basal ganglia information flow exist: one supporting parallel information flow from functionally different cortical regions through the basal ganglia (Alexander et al., 1986, Alexander and Crutcher, 1990, Groenewegen et al., 1990, Volkmann et al., 2010) and the other supporting integration of information coming from functionally different cortical regions (Percheron et al., 1984, Percheron and Filion, 1991). In line with the concept of parallel information processing, the STN has been divided into three distinct subdivisions: a dorsolateral motor part, a medial limbic part and a ventro-lateral associative part (Parent and Hazrati, 1995, Hamani et al., 2004, Temel et al., 2005).

Unfortunately, STN DBS does not only improve parkinsonian motor symptoms, but also often may affect mood and cognition of PD patients (Krack et al., 2003, RodriguezOroz et al., 2004, Visser-Vandewalle et al., 2005, Temel et al., 2006). The cognitive and psychiatric unwanted side effects are supposed to be a result of the stimulation of the non-motor parts of the STN (Temel et al., 2005) and could be prevented by selective stimulation of the motor area. To achieve this, it is mandatory to determine to what degree the motor and non-motor regions are segregated. For this purpose, using extracellular electrophysiological approaches, the organization of the STN motor and nonmotor subdivisions were analyzed in the normal and dopamine (DA) depleted rodent brain. We performed cortical stimulation and recorded STN neuronal responses to determine to what extent information flow from motor and non-motor cortical areas to the STN is segregated, or is being integrated through the monosynaptic cortico-subthalamic pathway and the indirect cortico-striato-pallido-subthalamic pathway and assessed the changes on subthalamic responses to cortical stimulation after DA depletion. 


\section{Material and Methods}

\section{Subjects}

Male Sprague Dawley rats (Centre d'Elevage Depré, Saint-Doulchard, France) weighing 250-400 g were kept at constant room temperature $\left(21.2^{\circ} \mathrm{C}\right)$ and relative humidity (60\%) and had ad libitum access to water and food. All animal experiments were carried out in accordance with European Communities Council Directive 2010/63/UE. The study received approval from the local Ethics Committee (Bordeaux, France).

\section{6-hydroxydopamine lesions}

Details of the surgical procedure have been described earlier (Delaville et al., 2012). In brief, one hour before surgery rats were injected with desipramine $(25 \mathrm{mg} / \mathrm{kg}$, i.p., Sigma-Aldrich, Saint-Quentin Fallavier, France). Rats were anesthetized with ketamine (75 $\mathrm{mg} / \mathrm{kg}$ )/xylazine $(10 \mathrm{mg} / \mathrm{kg}$ ) and fixed in a stereotactic apparatus (Stoelting, Wood Dale, USA). The skull was exposed, and burr holes were made. Next, 6-OHDA $(2.5 \mu \mathrm{g} / \mu \mathrm{l}$, in sterile $0.9 \% \mathrm{NaCl}$ ) containing $0.1 \%$ ascorbic acid was unilaterally injected in the medial forebrain bundle at the following coordinates relative to Bregma (in mm): AP -2.8, ML -2, DV -8.4 (Paxinos and Watson, 1998). At the end of the injection, the cannula remained in place for an additional 2 minutes and was then withdrawn slowly from the brain to prevent reflux of the solution.

\section{Single-unit recordings of STN neurons}

Rats were anesthetized with urethane hydrochloride (1.2 g/kg i.p., Sigma-Aldrich) and fixed in a stereotaxic apparatus (Horsley Clarke apparatus, Unimécanique, Epinay sur Seine, France). Body temperature was monitored with a rectal probe and maintained at $37^{\circ} \mathrm{C}$ with a homeothermic warming blanket (model 50-7061, Harvard Apparatus, Les Ulis, France). Burr holes were made for stimulation and recording sites.

Extracellular recordings were performed with a glass micropipette, impedance 8-12 MOhms, containing $4 \%$ pontamine sky blue dye dissolved in $0.9 \% \mathrm{NaCl}$. The pipette was lowered into the STN to record single-unit activity. The initial stereotactic coordinates relative to Bregma (in $\mathrm{mm}$ ) were: $\mathrm{AP}-3.8, \mathrm{ML}+/-2.5$ ). During penetration of the brain at the Bregma level of the STN, a characteristic burst firing of units was encountered in the thalamus. Passing through a silent zone before an area with spontaneous firing neurons was encountered. From the first trajectory the recording electrode was moved in the medio-lateral and/or anterio-posterior plane to acquire neural responses in all STN subareas. Action potentials were amplified with a differential preamplifier (GRASS P15F, West Warwick, USA) and a differential amplifier (AM systems 1700, Sydney, Australia). The signal was displayed on a memory oscilloscope and registered on a computer (Scope 3.6; MacLab 4s AD Instruments, Cambridge Electronic Design, UK). Spikes were 
separated from noise by a window discriminator apparatus (model 121, World Precision Instruments, UK) and sampled on-line (Spike 2 software, Cambridge Electronic Design, UK). At the end of each session, the last recording site used was marked by electrophoretic injection of pontamine sky blue.

\section{Electrical stimulation of the prefrontal cortices}

A construction of two homemade, concentric electrodes was used for stimulation of five cortical regions ipsilateral to the recording site, namely the motor $(\mathrm{MC})$, cingulate $(\mathrm{Cg})$, prelimbic (PrL), infralimbic (IL) and the agranular insular cortices (AI) (Tan et al., 2010). Stereotactic coordinates (in $\mathrm{mm}$ ) relative to Bregma were respectively: AP 3.2, ML 4.0, VD -2.6 (MC); AP 3.2, ML 0.6, VD -2.6 (Cg); AP 3.2, ML 0.6, VD -3.8 (PrL); AP 3.2, ML 0.6, VD -5.2 (IL); AP 3.2, ML 4.0, VD -5.2 (Al) (Paxinos and Watson, 1998) (Fig. 1). Electrical stimuli were generated with an isolated stimulator (DS3, Digitimer Ltd., Hertfordshire, UK) triggered either by a MacLab/4S system (AD Instruments, Castle Hill, Australia). Stimulation consisted of 200 monophasic pulses of $0.3 \mathrm{~ms}$ width and $300 \mu \mathrm{A}$ intensity delivered at a frequency of $1 \mathrm{~Hz}$. These stimulation parameters were chosen based on earlier studies (Fujimoto and Kita, 1993, Maurice et al., 1999, Kolomiets et al., 2001). The electrodes were moved up and down during the recordings to stimulate all targets. No qualitative differences in the response profiles were seen when moving the stimulation electrodes from the most dorsal cortical regions down to the most ventral cortical regions and back to the initial stimulation site. Histological evaluation of the brain sections, using acetylcholine esterase staining, confirmed that the electrodes traversed through the frontal cortex and reached a maximal depth of $5.2 \mathrm{~mm}$ according to bregma. Along the electrode trajectory, no additional histological damage was observed.

\section{Histology and immunohistochemistry}

At the end of experiments, animals were sacrificed, brains were collected and frozen in isopentane at $-45^{\circ} \mathrm{C}$ and stored at $-80^{\circ} \mathrm{C}$. Fresh-frozen brains were cryostat-cut on glass into coronal sections for localization of stimulation track in the cortex and pontamine sky blue dot in the STN. To this aim, acetylcholine esterase staining was used to contrast structures and to determine the location of the pontamine sky blue dot marking the last recording sites. Briefly, slides were air dried for one hour, rinsed for 5 minutes in a sodium acetate buffer solution ( $\mathrm{pH}$ 5.9). After pre-incubation for 15 minutes in a $0.375 \%$ glycine, $0.25 \%$ copper-sulfate sodium acetate buffer solution, slides were incubated in 200 $\mathrm{ml}$ 0.375\% glycine, $0.25 \%$ copper-sulfate sodium acetate buffer solution with $200 \mathrm{ml}$ acetylcholine iodide for 4 hours. Then, slides were washed for 5 minutes in the buffer solution, followed by a 2 minutes exposure to a $2 \%$ ammonium sulfide sodium acetate buffer solution. Finally, slides were rinsed 3 times in the buffer solution for 5 minutes. 


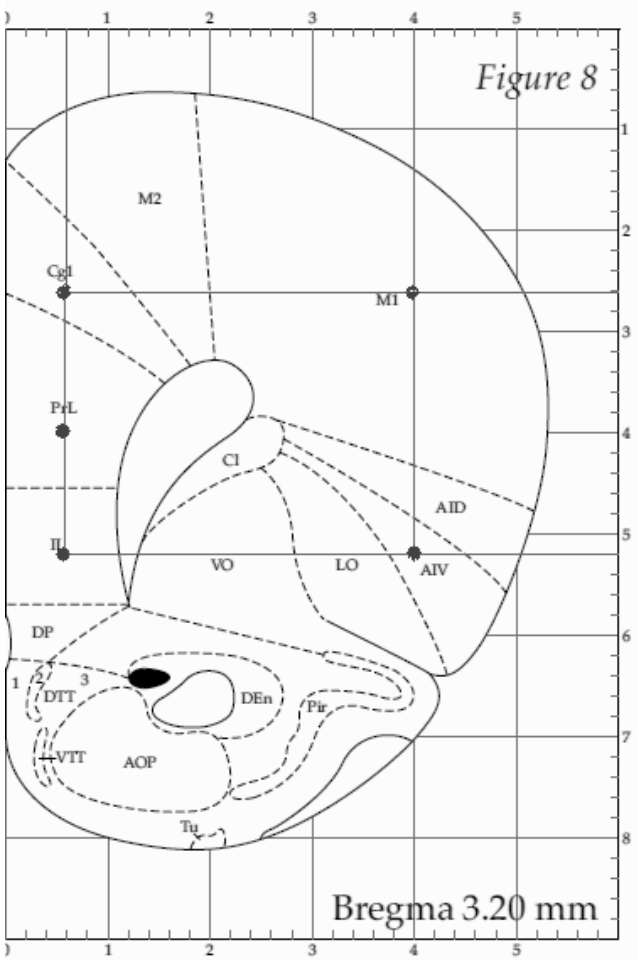

Fig. 1. Drawing of trajectory of cortical electrodes and the stimulation sites in the frontal cortex. The stimulation electrode construction was moved up and down along the same trajectory. Stimulation sites were the motor cortex, cingulate gyrus, infralimbic gyrus, prelimbic cortex and the agranular cortex.

\section{Biochemistry}

Animals for final analysis went through an additional validation step regarding the extent of DA depletion. This biochemical step was mandatory for the inclusion of the electrophysiology data from 6-OHDA animals. Monoamine tissue levels were measured by high-performance liquid chromatography (HPLC) coupled with electrochemical detection (De Deurwaerdere et al., 1998) to evaluate the extent of the DA depletion. Tissue concentrations of the dopamine and metabolites 3,4-Dihydroxyphenylacetic acid (DOPAC) and homovanillic acid (HVA) were measured in the anterior part of the striatum. At the end of electrophysiological recordings, rats were decapitated, and their brains were removed rapidly and frozen in cold isopentane. Both right and left portions of the anterior striatum were dissected and stored at $-80{ }^{\circ} \mathrm{C}$ until their use in biochemical assays. The tissues were homogenized in $200 \mu \mathrm{l}$ of $0.1 \mathrm{~N} \mathrm{HClO} 4$ and centrifuged at 13,000 rpm for $30 \mathrm{~min}$ at $4{ }^{\circ} \mathrm{C}$. Aliquots of the supernatants were diluted in the mobile phase and injected into the HPLC column (Chromasyl C8, 150×4.6 mm, $5 \mu \mathrm{m}$ ) protected by a Brownlee-Newgard precolumn (RP-8, 15×3.2 mm, $7 \mu \mathrm{m})$. The mobile phase, delivered at $1.2 \mathrm{ml} / \mathrm{min}$ flow rate, was as follows (in $\mathrm{mM}$ ): $60 \mathrm{NaH} 2 \mathrm{PO} 4,0.1$ disodium EDTA, and 2-octane sulfonic acid plus 7\% methanol, adjusted to $\mathrm{pH} 3.9$ with orthophosphoric acid and filtered through a $0.22-\mu \mathrm{m}$ Millipore filter. Detection of monoamines was per- 
formed with a coulometric detector (Coulochem I, ESA, Knivsta, Sweden) coupled to a dual-electrode analytic cell (model 5011). The potential of the electrodes was set at +350 and $-270 \mathrm{mV}$. Paired-sample T-tests were performed between the left and right side of the brain samples using the Statistical Package for the Social Sciences version 22.0 software (SPSS Inc, Chicago, Illinois, USA).

\section{Data analysis}

The basal firing rates and firing patterns of STN neurons were determined from the first 10 minutes of recording before stimulation of the cortical areas was started. The mean firing rate was calculated per neuron and a discharge density histogram was developed to assess the firing pattern using the Kaneoke and Vitek method (Kaneoke and Vitek, 1996, Chetrit et al., 2009, Janssen et al., 2012). The firing rate was compared between control and 6-OHDA animals using a t-test, firing patterns were analyzed using a Chisquare test. Peristimulus time histograms (PSTHs) were constructed from 200 consecutive stimulation trials with a bin size of $1 \mathrm{~ms}$ for each cortical stimulation site $(\mathrm{MC}, \mathrm{Cg}$, $\mathrm{PrL}, \mathrm{IL}$ and $\mathrm{Al}$ ). The criterion used to establish the existence of an excitatory response corresponded to the mean of the number of spikes recorded during the 500 ms preceding the onset of cortical stimulation plus the standard deviation (SD) of this mean. An inhibition was considered as a period during which the number of spikes is $50 \%$ below the mean of the number of spikes during the 500 ms period preceding the stimulation or if no spikes were recorded (Georges and Aston-Jones, 2002, Beyeler et al., 2010, Kita and Kita, 2011). Figures were made with Adobe Photoshop CS3 Version: 10.0.1 (Adobe, San Jose, USA) and Matlab (The MathWorks, Natick, USA).

\section{Results}

\section{Subthalamic neuronal responses to cortical stimulation in control animals}

A total of 284 STN neurons were recorded in control animals. The spontaneous firing rate in control animals was $8.6 \pm 0.45$ spikes/sec (mean \pm SEM) (figure 2). The firing pattern was regular in XX\% of the STN recorded neurons, and irregular or bursty in XX\% of the neurons. $79 \%$ of the subthalamic neurons responded to stimulation of the MC, CG, PrL, IL and/ or Al cortices. Of all the STN neurons recorded in control animals, 37\% showed a triphasic response, $10 \%$ had only an early excitation, while $37 \%$ had only a late excitation and 3\% a combination of the early and late excitation, 13\% showed only the STN specific long-lasting inhibition. These response patterns are in line with previous reports (Kitai and Deniau, 1981, Ryan and Clark, 1991, Fujimoto and Kita, 1993, Maurice et al., 1998, Kita and Kita, 2011). 
A

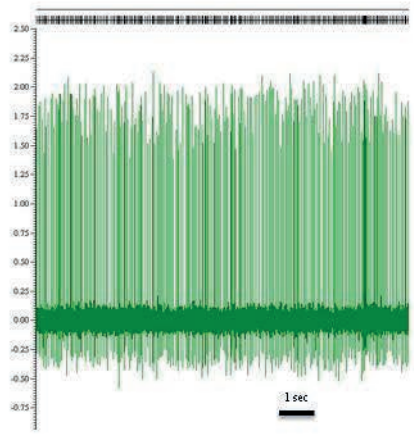

B

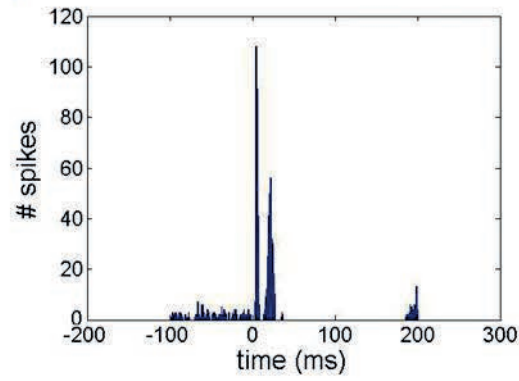

C

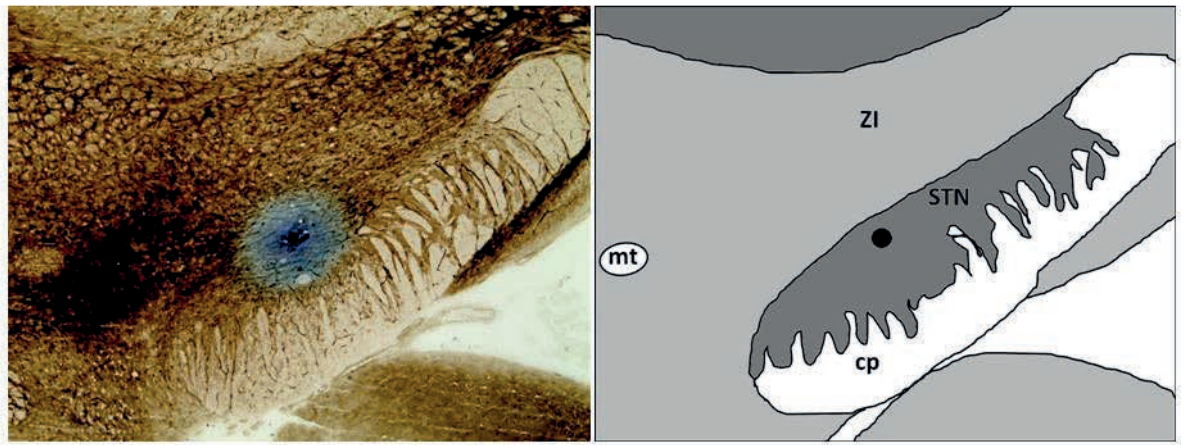

Fig. 2. An example is shown of a baseline recording of a regular firing subthalamic neuron (A). Next a representative triphasic response is shown in a peristimulus time histogram. First an early exaltation (E1) is seen followed by a late excitation (E2), between these excitatory phases, a short inhibition (I1) is present. After the triphasic response a long-lasting inhibition (I2) is seen (B). Also an example of iontophoretic blue point in the subthalamic nucleus is shown which was used to identify the exact anatomical localization of the recorded neurons (C).

Spatial distribution of subthalamic neurons in relation to the corresponding cortical stimulation site.

Figure $2 \mathrm{C}$ shows an example of a pontamine sky blue dot marking the site of the last recorded neuron in the STN. The dots were used to reconstruct the location of the STN recorded neurons as shown in figure 3. STN neurons responsive to motor cortex stimulation were present throughout the whole STN, but more sparsely within the ventromedial part. Neurons responsive to stimulation of the associative-limbic cortices were more frequently found within the ventro-medial part of the STN. No differences were found in the localization of neurons showing a triphasic response, or just a response to either the direct monosynaptic cortico-subthalamic pathway or the indirect corticostriato-pallido-subthalamic pathway. Although a crude anatomical subdivision could be made between motor and non-motor areas within the STN, STN responses to motor cortex stimulation were not limited to the dorso-lateral part of the STN. The same, though to a lesser extent, was observed for responses to limbic/associative cortices. 


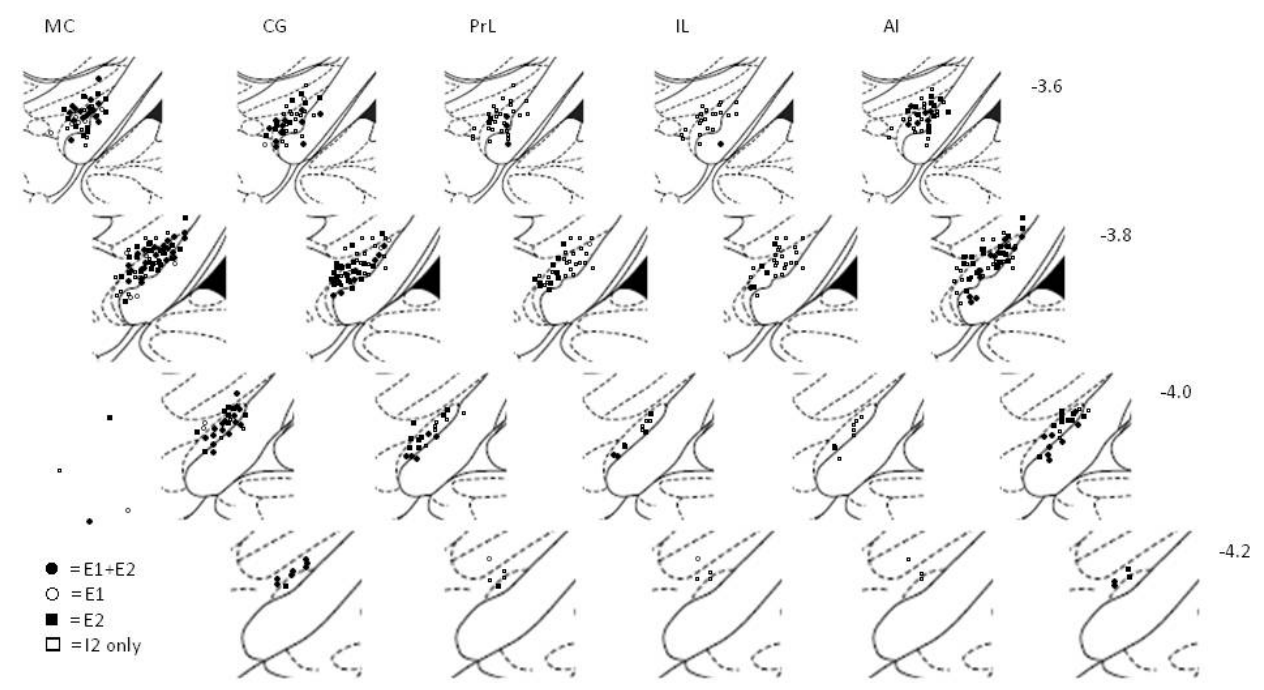

Fig. 3.Illustration of all localization of responsive and non responsive neurons per stimulation site, which were drawn relative to the last recorded neuron based on the iontophoretic blue point. It should be noticed that the neurons which responded to MC stimulation are mainly seen in the lateral parts of the STN. The neurons which responded to stimulation of the medial prefrontal cortical areas, for the most part the CG, were located in the medial part of the STN. The neurons which were responsive to the agranular insular cortex seemed to be mainly located in the dorsal border of the STN. Abbreviations: agranular insular cortex (AI), cingulate gyrus $(C G)$, dorsal (D), infralimbic cortex (IL), medial (M), motor cortex (MC), lateral (L), prelimbic cortex $(\operatorname{PrL})$, ventral (V).

Figure 4 illustrates representative examples of peristimulus time histograms of two neurons recorded one below another. The first neuron showed a triphasic response to MC, PrL and Al stimulation, whilst the second one showed a late excitation to CG and PrL stimulation, and to a lesser extent also to the MC. From the 284 recorded neurons, in total 513 neuronal responses from the STN were acquired by stimulation of the different cortical areas. In control animals most neurons responded to only one cortical region. $41 \%$ of STN neurons which were responsive to MC stimulation, did not respond to stimulation of the other stimulated cortical areas, nevertheless $13.6 \%$ of the neurons showed an early response to both $\mathrm{MC}$ and $\mathrm{Al}$, which originates from the monosynaptic cortico-subthalamic pathway, even more neurons responded with a late excitation to stimulation of different cortical regions. In table $1 \mathrm{~A}-\mathrm{C}$, the responses of STN neurons per cortical stimulation site are given. This displays the integration of motor and non-motor cortico-subthalamic pathways. In a few neurons we tested if a combined stimulation of $M C$ and $C G$ would change the amplitude of the triphasic response when the neuron responded to separate stimulation of these cortical areas (figure 5). No increase in the amplitude of the early or late excitation was seen. Although, the onset and duration of the excitatory phases was more stringent when stimulation was combined. 


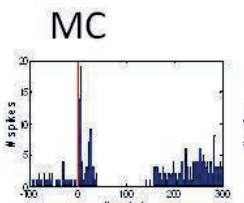

AP -3.8 , ML 2.5

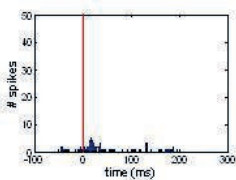

AP $-3.8, M L 2.5$
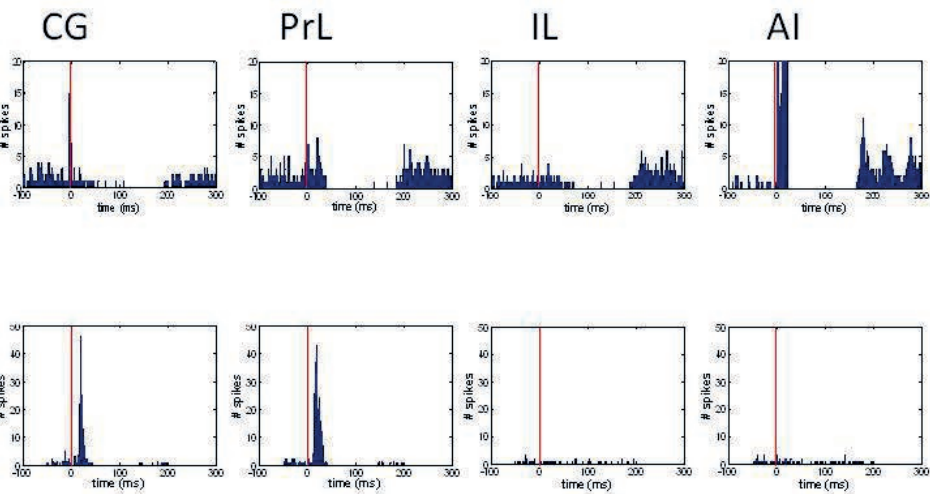

Fig. 4. A representative example of the responses to cortical stimulation of two neurons is shown. The second neuron was recorded just below the first one. The first neuron showed a triphasic response to MC, $\operatorname{PrL}$ and $\mathrm{Al}$ stimulation. The second neuron showed a late excitation to CG and PrL stimulation, and to a lesser extent also to the MC.

A

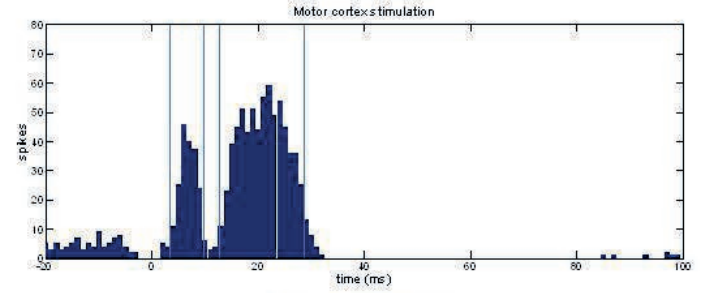

B

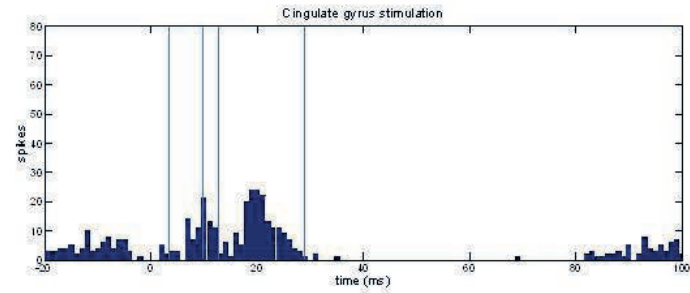

$\mathrm{C}$

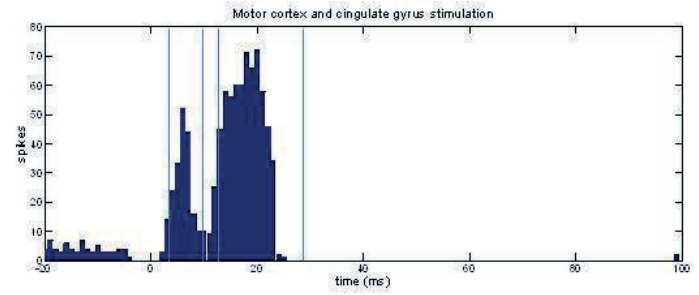

Fig. 5. Example of a neuronal response to stimulation of the $M C(A)$, the $C G(B)$, and the $M C$ and $C G$ combined (C). Abbreviations: cingulate gyrus (CG), motor cortex (MC). No increase in the amplitude of the early or late excitation was seen. Although, the onset and duration of the excitatory phases was shorter when stimulation was combined. 
Table 1. First the responses per stimulation site are displayed for control animals (A) and 6-OHDA animals (B); most neuronal responses were induced by $M C$ stimulation. Next the number of neurons which showed a response to different cortical areas is given for respectively the control (C) and 6-OHDA animals (D). Most overlap was seen between $\mathrm{MC}$ and $\mathrm{Al}$ stimulation, but also between $\mathrm{MC}$ and $\mathrm{CG}$ a significant number of STN neurons responded to stimulation of both areas. It should also be noticed that most overlap is seen in the E2 response from the excitatory responses

A

\begin{tabular}{cccccccc}
\hline & & triphasic & E1/E2 & E2 & E1 & inhibition only & total \\
\hline area & MC & 55 & 5 & 36 & 20 & 24 & 140 \\
& CG & 5 & 3 & 40 & 3 & 58 & 109 \\
& PrL & 0 & 1 & 16 & 1 & 52 & 70 \\
& IL & 0 & 0 & 9 & 0 & 58 & 67 \\
& $\mathrm{Al}$ & 33 & 4 & 38 & 3 & 48 & 127 \\
\hline Total & 87 & 13 & 96 & 15 & 240 & 513 \\
\hline
\end{tabular}

B

\begin{tabular}{llllllll}
\hline & & triphasic & E1/E2 & E2 & E1 & inhibition only & total \\
\hline area & MC & 61 & 7 & 35 & 19 & 14 & 136 \\
& CG & 8 & 4 & 37 & 4 & 34 & 87 \\
& PrL & 2 & 1 & 15 & 1 & 33 & 52 \\
& IL & 0 & 0 & 6 & 0 & 34 & 40 \\
& $\mathrm{Al}$ & 41 & 4 & 36 & 4 & 27 & 112 \\
\hline Total & 112 & 16 & 129 & 28 & 142 & 427 \\
\hline
\end{tabular}

C

\begin{tabular}{lrrl}
\hline & E1 & E2 & I2 \\
\hline MC-CG & 0 & 8 & 55 \\
MC-PrL & 0 & 6 & 44 \\
MC-IL & 0 & 2 & 45 \\
MC-AI & 28 & 53 & 75 \\
CG-PrL & 1 & 13 & 55 \\
CG-IL & 0 & 5 & 43 \\
CG-AI & 0 & 4 & 49 \\
PrL-IL & 0 & 6 & 40 \\
PrI-AI & 1 & 6 & 42 \\
IL-AI & 0 & 11 & 42 \\
\hline
\end{tabular}

D

\begin{tabular}{llll}
\hline & E1 & E2 & I2 \\
\hline MC-CG & 1 & 9 & 31 \\
MC-PrL & 0 & 8 & 28 \\
MC-IL & 0 & 4 & 27 \\
MC-Al & 36 & 52 & 72 \\
CG-PrL & 4 & 13 & 36 \\
CG-IL & 0 & 3 & 30 \\
CG-AI & 1 & 5 & 27 \\
PrL-IL & 0 & 2 & 23 \\
Prl-Al & 0 & 3 & 22 \\
IL-AI & 0 & 2 & 22 \\
\hline
\end{tabular}


Subthalamic neuronal responses to cortical stimulation in 6-OHDA-lesioned animals A total of 101 STN neurons were recorded in 6-OHDA lesioned animals. Dopamine depletion was confirmed in the 6-OHDA treated animals $(p<0.01)$. Table 2 summarizes the tissue content of DA, DOPAC and HVA in the anterior striatum. Compared to control animals, the spontaneous firing rate of STN neurons in DA depleted animals significantly increased to $12.8 \pm 1.12 \mathrm{~Hz}$ (mean $\pm \mathrm{SEM}, \mathrm{F}=10.0, \mathrm{p}=0.002$ ). The firing pattern of STN neurons 6-OHDA lesioned animals was in $44 \%$ of the neurons regular; $56 \%$ of the neurons showed an irregular or bursty firing pattern, which is in correspondence to previous studies (Hollerman and Grace, 1992, Ni et al., 2001, Janssen et al., 2012). 88\% of the subthalamic neurons responded to stimulation of the MC, CG, PrL, IL and Al. Of all the STN neurons recorded in DA depleted animals, 39\% showed a triphasic response, $3 \%$ had only an early excitation, while $25 \%$ had only a late excitation and $13 \%$ a combination of the early and late excitation, 9\% showed only the STN specific long-lasting inhibition. Interestingly, an increased number of neurons from the responsive neurons showed an excitation compared to the control group. This increase was seen in the group of responses in which a combined early and late excitation was present without early inhibition in-between. However, these differences were not significantly different between normal and DA depleted animals using a Chi-square test $(p=0.22)$. The same overlap of responses to the stimulation of different cortical areas was seen in 6-OHDA lesioned animals compared to control animals (Table 1B-D). The anatomical localization of neurons within the STN depending on the responsiveness to stimulation of a specific cortical area was the same for normal and DA depleted rats.

Table 2.

\begin{tabular}{lccccc}
\hline & \multicolumn{2}{c}{ Control } & & \multicolumn{2}{c}{ Unilateral 6-OHDA lesion } \\
\cline { 2 - 3 } \cline { 5 - 6 } & left & right & & left & right \\
\hline Dopamine & $13598 \pm 1367$ & $13572 \pm 1149$ & & $12079 \pm 375$ & $1601 \pm 480^{* *}$ \\
Noradrenaline & $131 \pm 11$ & $132 \pm 10$ & & $95 \pm 12$ & $18 \pm 5^{* *}$ \\
Serotonin & $195 \pm 21$ & $233 \pm 27$ & & $240 \pm 34$ & $181 \pm 29$ \\
\hline
\end{tabular}

Neurochemical analysis of unilateral 6-OHDA treatment induced depletion of DA. Tissue contents of striatal DA, 3,4-Dihydroxyphenylacetic acid (DOPAC) and homovanillic acid (HVA) measured by HPLC are depicted. Values are concentrations in $\mathrm{ng} / \mathrm{g}$ of wet tissue presented as the mean \pm SEM. Paired-sample t-test was performed between the left and right side of the brain samples. ${ }^{*} p<0.05,{ }^{*} p<0.01$. Abbreviations: dopamine (DA), 3,4-Dihydroxyphenylacetic acid (DOPAC) and homovanillic acid (HVA).

\section{Discussion}

Here we provided the first robust evidence showing that motor and non-motor corticosubthalamic pathways in the rat are not fully segregated, but partially integrated in the rat. This integration was most present through the indirect pathway. 
Our results showed that most STN neurons were responsive to cortical stimulation. From the responsive neurons, the majority responded to stimulation of two or more cortical areas. Interestingly, responses to different cortical areas were mainly seen with a long latency. These long latency responses are related to the 'indirect' cortico-striatopallido-subthalamic pathway. A substantial number of neurons also showed a short latency response mainly originating from motor cortex, but was also seen after the stimulation of non-motor cortical areas. This short latency response is caused by the excitation of STN neurons through the monosynaptic glutamatergic cortico-subthalamic pathway. Frequently a combination of short and long latency responses were observed, which have been earlier characterized as a triphasic response (Fujimoto and Kita, 1993, Nambu et al., 2000, Kolomiets et al., 2001, Magill et al., 2004).

A large number of STN neurons selectively responded to either motor or nonmotor cortical regions with a crude somatotopy. Indeed, the responsive neurons to motor cortex stimulation were mainly located in the lateral portion of the STN, whereas neurons responding to stimulation of limbic/associative cortical areas were mainly seen in the medial parts of the STN. On the contrary, it should also be noticed that responsive neurons to the stimulation of motor and non-motor cortices were not limited to these areas and functional territories largely overlapped. Indeed, a significant number of STN neurons responded to stimulation of both motor and non-motor regions, with a long latency response and also with short latency responses. Therefore, our findings are not in line with the two major theories for cortico-basal ganglia information processing. They do not support the absolute, parallel flow of motor, associative and limbic information through the cortico-basal ganglia circuits (Alexander et al., 1986, Alexander and Crutcher, 1990, Groenewegen et al., 1990, Volkmann et al., 2010) and neither do these results support a pure convergence of these pathways (Percheron et al., 1984, Percheron and Filion, 1991). Our findings, however, are in agreement with another theory which presumes parallel cortico-basal ganglia pathways with interaction between them (Joel and Weiner, 1994). The concept that the cortico-basal ganglia circuits are not completely closed and segregated from each other has accompanied the principle of parallel organization in the first description of this network (Alexander et al., 1986). A review of anatomical studies showed that the cortico-basal ganglia network is interconnected by pathways, which are themselves components of the different loops within this network (Joel and Weiner, 1994). Thus, with respect to our results the organization of the cortico-subthalamic network is best described as functionally interconnected, parallel loops. There are several arguments supporting this vision: first of all dendrites extend across almost the entire STN (Hammond and Yelnik, 1983). Second, an anatomical tracer study has shown highly overlapped and fuzzy borders of somatopic presentations in the subthalamic nucleus, in agreement with our electrophysiological functional findings (Kita et al., 2014). The overlap between functional territories is also described 
in non-human primates. Overlap is seen in projections from prefrontal, pre-motor, motor and limbic projections to the STN (Nambu et al., 1996, Haynes and Haber, 2013), and the same overlap is seen from projections from the GPe (Kita, 2007). An even stronger argument is that responses to stimulation of the non-motor cortices have been seen in STN neurons, which also responded to motor cortex stimulation (Kolomiets et al., 2001). Moreover, when we stimulated two different cortical areas the neuronal response was modulated. Other strong afferent projections of the STN from the ventral pallidum, intralaminar thalamic nuclei, and pedunculopontine tegmental nucleus also show overlapped functional anatomical projections (Groenewegen et al., 1993, Maurice et al., 1998). It is also not surpising that the cortico-subthalamic pathways are not organized in strict parallel matter since interactions between associative, limbic and motor circuits are necessary to perform complex tasks in stressful and highly demanding circumstances.

These findings are also clinically relevant. In Parkinson's disease, STN DBS is applied to improve motor symptoms. Therefore the dorso-lateral sensori-motor part of the STN is targeted. Many efforts are currently made to improve targeting to avoid undesired neuropsychiatric side-effects, which are thought to be caused by stimulation of the nonmotor regions of the STN. If the interconnectivity of cortico-subthalamic pathways is as strong in humans as in rats, then these side effects cannot be totally avoided.

In the 6-OHDA treated rats no differences were seen in the functional anatomical localization of STN neurons compared to the sham group. So again a crude somatotopy was seen, but with great overlap. This finding shows that in a dopamine depleted state the somatotopy of STN neurons remains unchanged. Interestingly, as shown in table 1 the interconnectivity of cortico-subthalamic pathways was similar in the control and 6OHDA treated group. It can however not be ruled out that in a long-term dopamine depleted brain segregation of cortico-subthalamic pathways is vanishing even further.

\section{Conclusion}

In this study, we show that the neuronal responses to cortical stimulation of the STN are grossly anatomically organized with large overlap and interaction between different functional cortico-subthalamic pathways is seen electrophysiologically. For STN DBS in PD patients this could suggest that avoidance of behavioral side effects by selective stimulation of the motor part solely is hard to achieve, since a pure motor part may not exist. 


\section{Acknowledgements}

The authors gratefully acknowledge the support of the BrainGain Smart Mix Programme of the Netherlands Ministry of Economic Affairs and the Netherlands Ministry of Education, Culture and Science (grant no.: SSM06011). The study was also supported by the "Bordeaux University" and the "Centre National de la Recherche Scientifique" (CNRS, France). M. Janssen received travel grants from the Boehringer Ingelheim Foundation and the Dutch Parkinson Association. C. Delaville was supported by a fellowship from the "Ministère de l'Education Nationale, de la Recherche et de la Technologie" (France). We thank L. Cardoit for her technical assistance. The authors declare no competing financial interests. 


\section{References}

1. Albin, R. L., Young, A. B., and Penney, J. B., 1989. The functional anatomy of basal ganglia disorders. Trends Neurosci 12, 366-375.

2. Alexander, G. E., and Crutcher, M. D., 1990. Functional architecture of basal ganglia circuits: neural substrates of parallel processing. Trends Neurosci 13, 266-271.

3. Alexander, G. E., DeLong, M. R., and Strick, P. L., 1986. Parallel organization of functionally segregated circuits linking basal ganglia and cortex. Annu Rev Neurosci 9, 357-381.

4. Benazzouz, A., Boraud, T., Feger, J., Burbaud, P., Bioulac, B., and Gross, C., 1996. Alleviation of experimental hemiparkinsonism by high-frequency stimulation of the subthalamic nucleus in primates: a comparison with L-Dopa treatment. Movement disorders : official journal of the Movement Disorder Society 11, 627-632.

5. Benazzouz, A., Gross, C., Feger, J., Boraud, T., and Bioulac, B., 1993. Reversal of rigidity and improvement in motor performance by subthalamic high-frequency stimulation in MPTP-treated monkeys. Eur J Neurosci 5, 382-389.

6. Beyeler, A., Kadiri, N., Navailles, S., Boujema, M. B., Gonon, F., Moine, C. L., Gross, C., and De Deurwaerdere, P., 2010. Stimulation of serotonin2C receptors elicits abnormal oral movements by acting on pathways other than the sensorimotor one in the rat basal ganglia. Neuroscience 169, 158-170.

7. Chetrit, J., Ballion, B., Laquitaine, S., Belujon, P., Morin, S., Taupignon, A., Bioulac, B., Gross, C. E., and Benazzouz, A., 2009. Involvement of Basal Ganglia network in motor disabilities induced by typical antipsychotics. PLoS One 4, e6208.

8. De Deurwaerdere, P., Stinus, L., and Spampinato, U., 1998. Opposite change of in vivo dopamine release in the rat nucleus accumbens and striatum that follows electrical stimulation of dorsal raphe nucleus: role of 5-HT3 receptors. The Journal of neuroscience : the official journal of the Society for Neuroscience 18, 6528-6538.

9. Degos, B., Deniau, J. M., Le Cam, J., Mailly, P., and Maurice, N., 2008. Evidence for a direct subthalamocortical loop circuit in the rat. Eur J Neurosci 27, 2599-2610.

10. Delaville, C., Chetrit, J., Abdallah, K., Morin, S., Cardoit, L., De Deurwaerdere, P., and Benazzouz, A., 2012. Emerging dysfunctions consequent to combined monoaminergic depletions in Parkinsonism. Neurobiol Dis 45, 763-773.

11. Fujimoto, K., and Kita, H., 1993. Response characteristics of subthalamic neurons to the stimulation of the sensorimotor cortex in the rat. Brain Res 609, 185-192.

12. Georges, F., and Aston-Jones, G., 2002. Activation of ventral tegmental area cells by the bed nucleus of the stria terminalis: a novel excitatory amino acid input to midbrain dopamine neurons. The Journal of neuroscience : the official journal of the Society for Neuroscience 22, 5173-5187.

13. Groenewegen, H. J., Berendse, H. W., and Haber, S. N., 1993. Organization of the output of the ventral striatopallidal system in the rat: ventral pallidal efferents. Neuroscience 57, 113-142.

14. Groenewegen, H. J., Berendse, H. W., Wolters, J. G., and Lohman, A. H., 1990. The anatomical relationship of the prefrontal cortex with the striatopallidal system, the thalamus and the amygdala: evidence for a parallel organization. Prog Brain Res 85, 95-116; discussion 116-118.

15. Hamani, C., Saint-Cyr, J. A., Fraser, J., Kaplitt, M., and Lozano, A. M., 2004. The subthalamic nucleus in the context of movement disorders. Brain 127, 4-20.

16. Hammond, C., and Yelnik, J., 1983. Intracellular labelling of rat subthalamic neurones with horseradish peroxidase: computer analysis of dendrites and characterization of axon arborization. Neuroscience 8, 781-790.

17. Haynes, W. I., and Haber, S. N., 2013. The organization of prefrontal-subthalamic inputs in primates provides an anatomical substrate for both functional specificity and integration: implications for Basal Ganglia models and deep brain stimulation. The Journal of neuroscience : the official journal of the Society for Neuroscience 33, 4804-4814.

18. Hollerman, J. R., and Grace, A. A., 1992. Subthalamic nucleus cell firing in the 6-OHDA-treated rat: basal activity and response to haloperidol. Brain Res 590, 291-299. 
19. Janssen, M. L., Zwartjes, D. G., Tan, S. K., Vlamings, R., Jahanshahi, A., Heida, T., Hoogland, G., Steinbusch, H. W., Visser-Vandewalle, V., and Temel, Y., 2012. Mild dopaminergic lesions are accompanied by robust changes in subthalamic nucleus activity. Neurosci Lett 508, 101-105.

20. Janssen, M. L. F., Duits, A. A., Turaihi, A. H., Ackermans, L., Leentjens, A. F. G., van Kranen-Mastenbroek, V., Oosterloo, M., Visser-Vandewalle, V., and Temel, Y., 2014. Subthalamic nucleus high frequency stimulation for advanced Parkinson's disease: motor and neuropsychological outcome after 10 years. Stereotact Funct Neurosurg 92, 381-387.

21. Joel, D., and Weiner, I., 1994. The organization of the basal ganglia-thalamocortical circuits: open interconnected rather than closed segregated. Neuroscience 63, 363-379.

22. Kaneoke, Y., and Vitek, J. L., 1996. Burst and oscillation as disparate neuronal properties. J Neurosci Methods 68, 211-223.

23. Kita, H., 2007. Globus pallidus external segment. Prog Brain Res 160, 111-133.

24. Kita, H., and Kita, T., 2011. Cortical stimulation evokes abnormal responses in the dopamine-depleted rat basal ganglia. J Neurosci 31, 10311-10322.

25. Kita, T., Osten, P., and Kita, H., 2014. Rat subthalamic nucleus and zona incerta share extensively overlapped representations of cortical functional territories. J Comp Neurol 522, 4043-4056.

26. Kitai, S. T., and Deniau, J. M., 1981. Cortical inputs to the subthalamus: intracellular analysis. Brain Res 214, 411-415.

27. Kolomiets, B. P., Deniau, J. M., Mailly, P., Menetrey, A., Glowinski, J., and Thierry, A. M., 2001. Segregation and convergence of information flow through the cortico-subthalamic pathways. J Neurosci 21, 5764-5772.

28. Krack, P., Batir, A., Van Blercom, N., Chabardes, S., Fraix, V., Ardouin, C., Koudsie, A., Limousin, P. D., Benazzouz, A., LeBas, J. F., Benabid, A. L., and Pollak, P., 2003. Five-year follow-up of bilateral stimulation of the subthalamic nucleus in advanced Parkinson's disease. N Engl J Med 349, 1925-1934.

29. Limousin, P., Pollak, P., Benazzouz, A., Hoffmann, D., Broussolle, E., Perret, J. E., and Benabid, A. L., 1995. Bilateral subthalamic nucleus stimulation for severe Parkinson's disease. Mov Disord 10, 672-674.

30. Magill, P. J., Sharott, A., Bevan, M. D., Brown, P., and Bolam, J. P., 2004. Synchronous unit activity and local field potentials evoked in the subthalamic nucleus by cortical stimulation. J Neurophysiol 92, 700714.

31. Maurice, N., Deniau, J. M., Glowinski, J., and Thierry, A. M., 1998. Relationships between the prefrontal cortex and the basal ganglia in the rat: physiology of the corticosubthalamic circuits. J Neurosci 18, 95399546.

32. Maurice, N., Deniau, J. M., Glowinski, J., and Thierry, A. M., 1999. Relationships between the prefrontal cortex and the basal ganglia in the rat: physiology of the cortico-nigral circuits. J Neurosci 19, 4674-4681.

33. Miyachi, S., Lu, X., Imanishi, M., Sawada, K., Nambu, A., and Takada, M., 2006. Somatotopically arranged inputs from putamen and subthalamic nucleus to primary motor cortex. Neurosci Res 56, 300-308.

34. Nambu, A., Takada, M., Inase, M., and Tokuno, H., 1996. Dual somatotopical representations in the primate subthalamic nucleus: evidence for ordered but reversed body-map transformations from the primary motor cortex and the supplementary motor area. J Neurosci 16, 2671-2683.

35. Nambu, A., Tokuno, H., Hamada, I., Kita, H., Imanishi, M., Akazawa, T., Ikeuchi, Y., and Hasegawa, N., 2000. Excitatory cortical inputs to pallidal neurons via the subthalamic nucleus in the monkey. J Neurophysiol 84, 289-300.

36. Nambu, A., Tokuno, H., and Takada, M., 2002. Functional significance of the cortico-subthalamo-pallidal 'hyperdirect' pathway. Neurosci Res 43, 111-117.

37. Ni, Z. G., Bouali-Benazzouz, R., Gao, D. M., Benabid, A. L., and Benazzouz, A., 2001. Time-course of changes in firing rates and firing patterns of subthalamic nucleus neuronal activity after 6-OHDA-induced dopamine depletion in rats. Brain Res 899, 142-147.

38. Parent, A., and Hazrati, L. N., 1995. Functional anatomy of the basal ganglia. II. The place of subthalamic nucleus and external pallidum in basal ganglia circuitry. Brain Res Brain Res Rev 20, 128-154.

39. Paxinos, G., and Watson, C., 1998. The Rat Brain in Stereotaxic Coordinates. Academic Press, New York. 
40. Percheron, G., and Filion, M., 1991. Parallel processing in the basal ganglia: up to a point. Trends Neurosci 14, 55-59.

41. Percheron, G., Yelnik, J., and Francois, C., 1984. A Golgi analysis of the primate globus pallidus. III. Spatial organization of the striato-pallidal complex. J Comp Neurol 227, 214-227.

42. Rodriguez-Oroz, M. C., Zamarbide, I., Guridi, J., Palmero, M. R., and Obeso, J. A., 2004. Efficacy of deep brain stimulation of the subthalamic nucleus in Parkinson's disease 4 years after surgery: double blind and open label evaluation. J Neurol Neurosurg Psychiatry 75, 1382-1385.

43. Ryan, L. J., and Clark, K. B., 1991. The role of the subthalamic nucleus in the response of globus pallidus neurons to stimulation of the prelimbic and agranular frontal cortices in rats. Exp Brain Res 86, 641-651.

44. Smith, Y., Bevan, M. D., Shink, E., and Bolam, J. P., 1998. Microcircuitry of the direct and indirect pathways of the basal ganglia. Neuroscience 86, 353-387.

45. Tan, S., Vlamings, R., Lim, L., Sesia, T., Janssen, M. L., Steinbusch, H. W., Visser-Vandewalle, V., and Temel, Y., 2010. Experimental deep brain stimulation in animal models. Neurosurgery 67, 1073-1079; discussion1080.

46. Temel, Y., Blokland, A., Steinbusch, H. W., and Visser-Vandewalle, V., 2005. The functional role of the subthalamic nucleus in cognitive and limbic circuits. Prog Neurobiol 76, 393-413.

47. Temel, Y., Kessels, A., Tan, S., Topdag, A., Boon, P., and Visser-Vandewalle, V., 2006. Behavioural changes after bilateral subthalamic stimulation in advanced Parkinson disease: a systematic review. Parkinsonism Relat Disord 12, 265-272.

48. Visser-Vandewalle, V., van der Linden, C., Temel, Y., Celik, H., Ackermans, L., Spincemaille, G., and Caemaert, J., 2005. Long-term effects of bilateral subthalamic nucleus stimulation in advanced Parkinson disease: a four year follow-up study. Parkinsonism Relat Disord 11, 157-165.

49. Volkmann, J., Daniels, C., and Witt, K., 2010. Neuropsychiatric effects of subthalamic neurostimulation in Parkinson disease. Nature reviews. Neurology 6, 487-498. 


\section{CHAPTER 6}

\section{Subthalamic Neuronal Responses to Cortical Stimulation}

Janssen MLF, Zwartjes DGM, Temel Y, Kranen-Mastenbroek V, Duits A, Bour L, Veltink P, Heida T, Visser-Vandewalle V.

Mov Dis 2012 Mar;27(3):435-8. 


\section{Abstract}

Background: Deep brain stimulation of the subthalamic nucleus alleviates motor symptoms in Parkinson's disease patients. However, some patients suffer from cognitive and emotional changes. These side effects are most likely caused by current spread to the cognitive and limbic territories in the subthalamic nucleus. The aim of this study was to identify the motor part of the subthalamic nucleus to reduce stimulationinduced behavioral side effects, by using motor cortex stimulation.

Methods: We describe the results of subthalamic nucleus neuronal responses to stimulation of the hand area of the motor cortex and evaluate the safety of this novel technique.

Results: Responses differed between regions within the subthalamic nucleus. In the anterior and lateral electrode at dorsal levels of the subthalamic nucleus, an early excitation ( $\sim 45 \mathrm{~ms}$ ) and subsequent inhibition inhibition (45-105 ms) were seen. The lateral electrode also showed a late excitation ( 125-160 ms). Focal seizures were observed following motor cortex stimulation.

Conclusions: To prevent seizures the current density should be lowered, so that motor cortex stimulation evoked responses can be safely used during deep brain stimulation surgery. 


\section{Introduction}

Deep brain stimulation (DBS) of the subthalamic nucleus (STN) alleviates motor symptoms in Parkinson's disease (PD) patients.(Deuschl, et al., 2006, Rodriguez-Oroz, et al., 2004, Weaver, et al., 2009) However, in a substantial number of patients the improvement of motor symptoms is accompanied by cognitive and/or limbic alterations.(Berney, et al., 2002, Piasecki and Jefferson, 2004, Smeding, et al., 2006, Temel, et al., 2006, Witt, et al., 2008) These behavioral side effects are thought to be caused by stimulation of the associative and limbic areas in the STN.(Temel, et al., 2005) Therefore, the optimal target is the dorsolateral part of the STN, supposedly the STN motor area.(Hamani, et al., 2004) Optimization, to identify the motor part, is currently done by intra-operative neurophysiologic measurements, such as spontaneous neuronal firing, neuronal kinesthetic responses and beta-power in the local field potential.(Chen, et al., 2006, de Solages, et al., 2011, Gross, et al., 2006) Earlier, Nishibayashi et al. applied subdural motor cortex stimulation (MCS) in humans in order to identify the motor area of the globus pallidus internus and externus (Nishibayashi, et al., 2011). This report will provide insight in the cortically evoked responses of the human STN neurons. The aim of the study was to identify the STN motor area by using MCS in order to reduce stimulation induced behavioral side effects. In this study, we tested the feasibility of identifying the STN motor part by motor cortex stimulation and evaluated the safety of this novel approach.

\section{Patients and Methods}

\section{Patients}

The study was approved by the Medical Ethical Committee of the Maastricht University Medical Center and all patients gave written informed consent. Patients were informed about the additional burr hole, subdural placement of the stimulation electrode and its additional potential complications, like the risk of a bleeding or a seizure. In- and exclusion criteria were the same as for standard DBS STN. In total, five PD patients with an age ranging between 55 and 70 years old were enrolled in this study.

\section{Procedure}

The procedure and results described below are from the fifth patient, because the stimulation protocols used in the other patients did not result in a STN response due to saturation of the amplifier in the first two patients and suboptimal MCS protocols in the remaining two patients. (see table 1). The day before the DBS procedure transcranial magnetic stimulation (TMS) was performed to localize the hand area of the motor cor- 
tex. The stereotactic procedure was performed under local anesthesia. Preoperatively, the patient was loaded with $15 \mathrm{mg} / \mathrm{kg}$ Diphantoine intravenously in ninety minutes. A strip of four electrodes (AD-Tech, model TS04R-SP10X-000; Racine, USA) was placed in the subdural space through a burr hole posterolateral to the hand area (identified by TMS). The strip electrode position was verified by performing a MEP registration at the contralateral hand and arm and the stimulation amplitude threshold was obtained. Subsequently, five micro-electrodes (InoMed, MicroMacroElectrode) were simultaneously inserted towards the STN through a precoronal burr hole. After baseline recordings, cortical evoked neuronal activity was measured using a multiple channel registration system (InoMed, ISIS MER System; stimulation settings: bipolar, monophasic, 0.2 $\mathrm{ms}, 15 \mathrm{~mA}$ ). After acquiring the cortically evoked responses, surgery was continued according to the standard procedure (Medtronic, Columbia Heights, Minneapolis, USA, model 3389). (Temel, et al., 2007) On the left side, the standard surgical procedure was performed without cortical stimulation. Three to four days after surgery, the electrodes were connected to an internal pulse generator (Medtronic, Kinetra, Model 7428).

Table 1. The different motor cortex stimulation protocols used in all patients and the responsiveness of the subthalamic neurons to the applied protocol. The stimulation protocols which evoked a STN response were only used in the fifth patient.

\begin{tabular}{llll}
\hline Monopolar/ bipolar & Anodal/ cathodal & $\begin{array}{l}\text { Amplitude } \\
\text { (times MEP level) }\end{array}$ & STN response \\
\hline Monopolar & Anodal & 0.33 & No \\
& 0.5 & No \\
& 0.67 & No \\
& 1 & No \\
Cathodal & 1.5 & No \\
& 0.5 & No \\
& 1 & No \\
Bipolar & 0.33 & No \\
& 0.67 & Yes (partial) \\
& 1 & Yes \\
\hline
\end{tabular}

The stimulation protocols that evoked a STN response were only used in the fifth patient. MEP, motor-evoked potential; STN, subthalamic nucleus.

\section{Data analysis}

Data analysis was performed in Matlab (MathWorks, Natick, MA, USA). First, offset and drift were removed from the signal by a high-pass butterworth filter at $5 \mathrm{~Hz}$. Subsequently, the stimulation artifact was removed. To assess multi/single unit activity, each epoch was digitally filtered between 350 and $5000 \mathrm{~Hz}$. Spike detection was performed 
using the envelope method.(Dolan, et al., 2009) To obtain single unit activity, spike sorting was performed by computing the principal components, which were clustered using either K-means or the Gaussian mixture model and the expectation maximization algorithm.(Lewicki, 1998) After spike detection, peristimulus time histograms (PSTHs) from 100 ms before until 200 ms after stimulation were constructed from 200 sweeps, grouping all trials with a specific stimulation setting. Bins of $1 \mathrm{~ms}$ were used and bins 1 ms before and 2 ms after cortical stimulation were set to zero to avoid any remaining stimulation artifact to be mistaken for spikes. To determine significant excitatory and inhibitory responses from the PSTHs, changing points indicating increases and decreases of the PSTH were detected using the change point analyzer software. (Magill, et al., 2004, Taylor, 2000) The periods between two changing points were tested for having a significantly different firing rate compared to the $100 \mathrm{~ms}$ preceding stimulation. This was done using a two-tailed t-test with a 5\% significance level. STN borders were determined by the intra-operative observations of the neurophysiologist and the postoperative analysis of the MER recordings.

\section{Results}

The STN was entered at a depth of $2 \mathrm{~mm}$ above target and left at $2.5 \mathrm{~mm}$ below target on the anterior and lateral trajectories. The central trajectory was within the STN from $0.5 \mathrm{~mm}$ to $3.5 \mathrm{~mm}$ below target, while the medial trajectory did not go through the STN. The posterior channel was defect and could not be analyzed. We measured 8 neurons inside the STN at various locations in this patient. The neurons had an average firing rate of $47 \pm 25 \mathrm{~Hz}$. Four neurons had a bursting pattern, three neurons showed a random pattern and one neuron showed a regular firing pattern. (Benazzouz, et al., 2002, Kaneoke and Vitek, 1996) Statistically significant responses in the STN were observed when MCS was performed with a single monophasic pulse (0.2 ms duration) at $15 \mathrm{~mA}$ and bipolar settings. Excitations ranged from a $30 \%$ to a $103 \%$ increase in firing rate relatively to the $100 \mathrm{~ms}$ period preceding stimulation, while the inhibitory periods ranged from an $11 \%$ to a $76 \%$ decrease in firing rate (Fig. 1). After each cortical stimulus, a clear contraction of the contralateral hand musculature was observed. Both spontaneous unit activity and unit responses to cortical stimulation were recorded from target -1.5 until target $+2.5 \mathrm{~mm}$. Inside the STN, responses to MCS were found, while outside of the STN no responses were observed except for the medial electrode at 2.5 $\mathrm{mm}$ from target (Fig. 1). Responses varied between different depths and between different locations in the anterior-posterior and medio-lateral plane within the STN. 


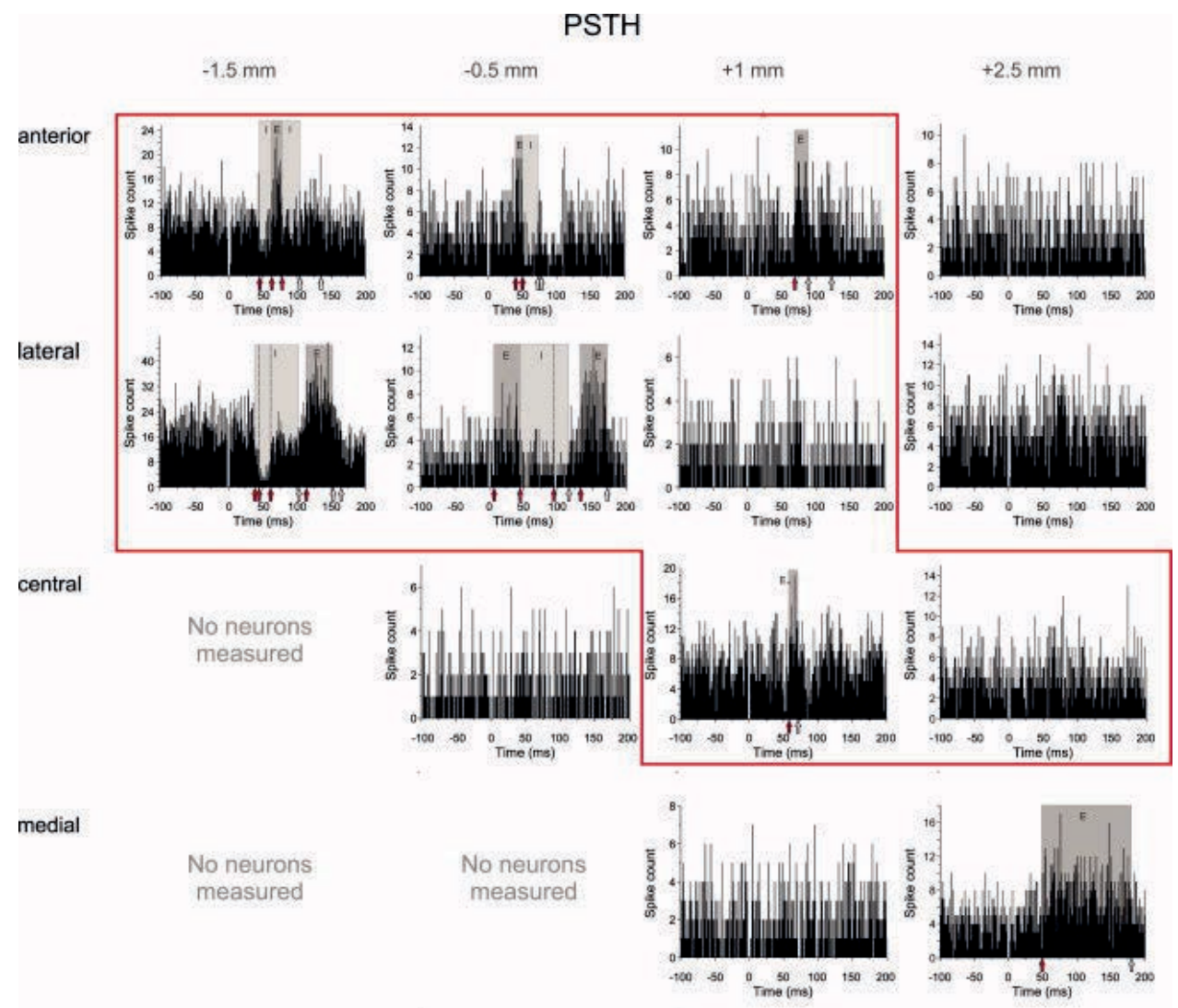

Fig. 1. PSTHs of neuronal recordings starting $0.5 \mathrm{~mm}$ after the electrode first enters the STN (1.5 $\mathrm{mm}$ above target) until the last electrode leaves the STN ( $2.5 \mathrm{~mm}$ below target). Recordings inside the STN are enclosed with a red window. MCS was performed with a bipolar electrode configuration using a monophasic pulse with an amplitude of $15 \mathrm{~mA}$ and a duration of $0.2 \mathrm{~ms}$. The arrows at the $\mathrm{x}$-axis indicate the significant changes that were identified with the change point analysis. The red arrows specify changes after which a significant increase or decrease in firing rate relatively to the 100-ms preceding stimulation was found. These periods are also indicated with shaded areas in which "I" denotes a period of inhibition, while "E" represents a period of excitation. When 2 periods of inhibition occurred after each other, a change is indicated with a dashed line. No results at $-1.5 \mathrm{~mm}$ on the medial and central electrode are shown, as no neurons were measured on these locations. MCS, motor cortex stimulation; PSTH, peristimulus time histogram; STN, subthalamic nucleus.

A focal seizure was seen in the first two patients; therefore Diphantoine was given preoperatively in the three following patients with approval of the Medical Ethical Committee. No seizures were present in patients 3 and 4 . Unfortunately, the fifth patient also had a focal seizure. In all patients, the seizure started in the contralateral hand area corresponding with the cortical stimulation side, with repetitive twitching. The seizure did not occur during stimulation, but with a latency period of more than one hour. The seizure could be controlled by acute application of additional i.v. anti-epileptic drugs. 
The anti-epileptics were stopped before discharge from the hospital. In the follow-up, no recurrent seizures occurred.

\section{Discussion}

The goal of this study was to provide insight in the cortically evoked responses of the human STN and evaluate the safety of this approach. We observed significant excitations and inhibitions as a response to MCS. Responses varied between different depths and between different locations in the anterior-posterior and medio-lateral plane within the STN. These responses can be used to identify the motor area of the STN. Selective DBS of the motor part of the STN has the potential to prevent unwanted behavioral side effects.

Studies in rats and primates showed typical triphasic responses, consisting of an initial excitation, a subsequent inhibition and a second excitation.(Fujimoto and Kita, 1993, Magill, et al., 2004, Maurice, et al., 1998, Nambu, et al., 2000) In contrast to intracortical stimulation electrodes in animal studies, we used flat stimulation electrodes placed on the cortical surface. It is likely that the difference in methodology is responsible for the lack of clear tri-phasic responses in human studies. (Strafella, et al., 2004) On the other hand, a contraction of the contralateral hand musculature was observed after each cortical stimulus, which indirectly proved that a significant number of pyramidal neurons in the hand area of the $M C$ were excited. A new finding is that in all electrode trajectories at different ventro-dorsal locations an "intermediate" excitation (starting from 63-79 ms) was present in the period of the long lasting inhibition. The most reasonable explanation for this third excitation is a sensory response of the STN to the muscular contraction induced by the MCS. (Hanajima, et al., 2004)

We believed that the burden of the affective and cognitive side effects outweighed the risks of the MCS procedure (additional burr hole, cortical stimulation). An important limitation of subdural MCS in our study is the occurrence of partial seizures. The risk of a seizure is related to the applied current and current density. In our stimulation protocol (settings: bipolar, monophasic, $0.2 \mathrm{~ms}, 15 \mathrm{~mA}, 1.1 \mathrm{~Hz}$ ), the current density was \pm 72 $\mu \mathrm{C} / \mathrm{cm}^{2}$, which might have been too high and thereby causing seizures. A second important consideration is the application of charge-balanced stimulation, which is achieved by biphasic instead of monophasic stimulation. Seizures also occur in other intra-operative procedures during which the cortex is stimulated repetitively (incidence of $1.2 \%$ ). (Szelenyi, et al., 2007) Interestingly, subdural MCS has been applied with a similar stimulation protocol without inducing epileptic seizures.(Nishibayashi, et al., 2011) The main difference between the stimulation protocols is that Nishibayashi et al. applied a lower number of stimuli and the electrode contact size was larger. To prevent 
seizures the current density should be lowered, so that MCS evoked responses can be safely used during DBS surgery.

\section{Acknowledgements}

This work was supported by the BrainGain Smart Mix Program of the Netherlands Ministry of Economic Affairs and the Netherlands Ministry of Education, Culture and Science [grant number SSM06011]. We thank J. Stegenga, M.A.J. Lourens, J. van der Meer and H.C.F. Martens for helpful discussions. 


\section{References}

1. Benazzouz, A., Breit, S., Koudsie, A., Pollak, P., Krack, P., and Benabid, A. L., 2002. Intraoperative microrecordings of the subthalamic nucleus in Parkinson's disease. Mov Disord 17 Suppl 3, S145-149.

2. Berney, A., Vingerhoets, F., Perrin, A., Guex, P., Villemure, J. G., Burkhard, P. R., Benkelfat, C., and Ghika, J., 2002. Effect on mood of subthalamic DBS for Parkinson's disease: a consecutive series of 24 patients. Neurology 59, 1427-1429.

3. Chen, C. C., Pogosyan, A., Zrinzo, L. U., Tisch, S., Limousin, P., Ashkan, K., Yousry, T., Hariz, M. I., and Brown, P., 2006. Intra-operative recordings of local field potentials can help localize the subthalamic nucleus in Parkinson's disease surgery. Exp Neurol 198, 214-221.

4. de Solages, C., Hill, B. C., Yu, H., Henderson, J. M., and Bronte-Stewart, H., 2011. Maximal subthalamic beta hypersynchrony of the local field potential in Parkinson's disease is located in the central region of the nucleus. J Neurol Neurosurg Psychiatry 82, 1387-1389.

5. Deuschl, G., Schade-Brittinger, C., Krack, P., Volkmann, J., Schafer, H., Botzel, K., Daniels, C., Deutschlander, A., Dillmann, U., Eisner, W., Gruber, D., Hamel, W., Herzog, J., Hilker, R., Klebe, S., Kloss, M., Koy, J., Krause, M., Kupsch, A., Lorenz, D., Lorenzl, S., Mehdorn, H. M., Moringlane, J. R., Oertel, W., Pinsker, M. O., Reichmann, H., Reuss, A., Schneider, G. H., Schnitzler, A., Steude, U., Sturm, V., Timmermann, L., Tronnier, V., Trottenberg, T., Wojtecki, L., Wolf, E., Poewe, W., and Voges, J., 2006. A randomized trial of deep-brain stimulation for Parkinson's disease. N Engl J Med 355, 896-908.

6. Dolan, K., Martens, H. C., Schuurman, P. R., and Bour, L. J., 2009. Automatic noise-level detection for extra-cellular micro-electrode recordings. Med Biol Eng Comput 47, 791-800.

7. Fujimoto, K., and Kita, H., 1993. Response characteristics of subthalamic neurons to the stimulation of the sensorimotor cortex in the rat. Brain Res 609, 185-192.

8. Gross, R. E., Krack, P., Rodriguez-Oroz, M. C., Rezai, A. R., and Benabid, A. L., 2006. Electrophysiological mapping for the implantation of deep brain stimulators for Parkinson's disease and tremor. Mov Disord 21 Suppl 14, S259-283.

9. Hamani, C., Saint-Cyr, J. A., Fraser, J., Kaplitt, M., and Lozano, A. M., 2004. The subthalamic nucleus in the context of movement disorders. Brain 127, 4-20.

10. Hanajima, R., Dostrovsky, J. O., Lozano, A. M., Hutchison, W. D., Davis, K. D., Chen, R., and Ashby, P., 2004. Somatosensory evoked potentials (SEPs) recorded from deep brain stimulation (DBS) electrodes in the thalamus and subthalamic nucleus (STN). Clin Neurophysiol 115, 424-434.

11. Kaneoke, Y., and Vitek, J. L., 1996. Burst and oscillation as disparate neuronal properties. Journal of Neuroscience Methods 68, 211-223.

12. Lewicki, M. S., 1998. A review of methods for spike sorting: the detection and classification of neural action potentials. Network 9, R53-78.

13. Magill, P. J., Sharott, A., Bevan, M. D., Brown, P., and Bolam, J. P., 2004. Synchronous unit activity and local field potentials evoked in the subthalamic nucleus by cortical stimulation. J Neurophysiol 92, 700714.

14. Maurice, N., Deniau, J. M., Glowinski, J., and Thierry, A. M., 1998. Relationships between the prefrontal cortex and the basal ganglia in the rat: physiology of the corticosubthalamic circuits. J Neurosci 18, 95399546.

15. Nambu, A., Tokuno, H., Hamada, I., Kita, H., Imanishi, M., Akazawa, T., Ikeuchi, Y., and Hasegawa, N., 2000. Excitatory cortical inputs to pallidal neurons via the subthalamic nucleus in the monkey. J Neurophysiol 84, 289-300.

16. Nishibayashi, H., Ogura, M., Kakishita, K., Tanaka, S., Tachibana, Y., Nambu, A., Kita, H., and Itakura, T., 2011. Cortically evoked responses of human pallidal neurons recorded during stereotactic neurosurgery. Mov Disord.

17. Piasecki, S. D., and Jefferson, J. W., 2004. Psychiatric complications of deep brain stimulation for Parkinson's disease. J Clin Psychiatry 65, 845-849. 
18. Rodriguez-Oroz, M. C., Zamarbide, I., Guridi, J., Palmero, M. R., and Obeso, J. A., 2004. Efficacy of deep brain stimulation of the subthalamic nucleus in Parkinson's disease 4 years after surgery: double blind and open label evaluation. J Neurol Neurosurg Psychiatry 75, 1382-1385.

19. Smeding, H. M., Speelman, J. D., Koning-Haanstra, M., Schuurman, P. R., Nijssen, P., van Laar, T., and Schmand, B., 2006. Neuropsychological effects of bilateral STN stimulation in Parkinson disease: a controlled study. Neurology 66, 1830-1836.

20. Strafella, A. P., Vanderwerf, Y., and Sadikot, A. F., 2004. Transcranial magnetic stimulation of the human motor cortex influences the neuronal activity of subthalamic nucleus. Eur J Neurosci 20, 2245-2249.

21. Szelenyi, A., Joksimovic, B., and Seifert, V., 2007. Intraoperative risk of seizures associated with transient direct cortical stimulation in patients with symptomatic epilepsy. J Clin Neurophysiol 24, 39-43.

22. Taylor, W. A., 2000. Change-Point Analyzer. Taylor Enterprises, Inc., Illinois, USA.

23. Temel, Y., Blokland, A., Steinbusch, H. W., and Visser-Vandewalle, V., 2005. The functional role of the subthalamic nucleus in cognitive and limbic circuits. Prog Neurobiol 76, 393-413.

24. Temel, Y., Kessels, A., Tan, S., Topdag, A., Boon, P., and Visser-Vandewalle, V., 2006. Behavioural changes after bilateral subthalamic stimulation in advanced Parkinson disease: a systematic review. Parkinsonism Relat Disord 12, 265-272.

25. Temel, Y., Wilbrink, P., Duits, A., Boon, P., Tromp, S., Ackermans, L., van Kranen-Mastenbroek, V., Weber, W., and Visser-Vandewalle, V., 2007. Single electrode and multiple electrode guided electrical stimulation of the subthalamic nucleus in advanced Parkinson's disease. Neurosurgery 61, 346-355; discussion 355-347.

26. Weaver, F. M., Follett, K., Stern, M., Hur, K., Harris, C., Marks, W. J., Jr., Rothlind, J., Sagher, O., Reda, D., Moy, C. S., Pahwa, R., Burchiel, K., Hogarth, P., Lai, E. C., Duda, J. E., Holloway, K., Samii, A., Horn, S., Bronstein, J., Stoner, G., Heemskerk, J., and Huang, G. D., 2009. Bilateral deep brain stimulation vs best medical therapy for patients with advanced Parkinson disease: a randomized controlled trial. JAMA 301, 63-73.

27. Witt, K., Daniels, C., Reiff, J., Krack, P., Volkmann, J., Pinsker, M. O., Krause, M., Tronnier, V., Kloss, M., Schnitzler, A., Wojtecki, L., Botzel, K., Danek, A., Hilker, R., Sturm, V., Kupsch, A., Karner, E., and Deuschl, G., 2008. Neuropsychological and psychiatric changes after deep brain stimulation for Parkinson's disease: a randomised, multicentre study. Lancet Neurol 7, 605-614. 


\section{CHAPTER 7}

\section{Cortically evoked potentials in the human subthalamic nucleus}

Janssen MLF, Zwartjes DGM, Heida T,

Van Kranen-Mastenbroek V, Bour LJ, Temel Y, Visser-Vandewalle V, Veltink PH

Neuroscience Letters 2013 Feb 28;539:27-31. 


\section{Abstract}

Deep brain stimulation (DBS) of the subthalamic nucleus (STN) alleviates motor symptoms in Parkinson's disease (PD) patients. However, in a substantial number of patients the beneficial effects of STN DBS are overshadowed by psychiatric side effects. We hypothesize that stimulation of the STN motor area will provide the optimal effect on the motor symptoms without inducing these side effects, and expect that motor cortex stimulation (MCS) evokes a spatially specific response within the STN, which identifies the STN motor area. We previously showed that MCS evokes responses in the unit activity specifically within certain areas of the STN. Unit activity is generally considered a measure of the output activity. To gain more insight into the neuronal input into the STN, we describe the results of cortically evoked subthalamic local field potentials (LFPs). We show that the cortically evoked LFPs follow a certain temporal and spatial pattern. The significant peaks of the evoked LFPs coincide with the timing of some of the inhibitions and excitations present in the unit responses. The spatial resolution of responses measured in the LFP to MCS is not high enough to identify the STN motor region. However, we believe that optimizing targeting techniques and the development of novel DBS electrodes will improve STN DBS therapy for PD patients. 


\section{Introduction}

Neuronal recordings from the human subthalamic nucleus (STN) have become possible due to the surgical treatment for advanced Parkinson's disease (PD), such as deep brain stimulation (DBS) of the STN. STN DBS provides a remarkable improvement in the motor function of PD patients (Deuschl, et al., 2006). Unfortunately, STN DBS also induces unwanted behavioral changes, such as emotional disturbances and cognitive alterations (Temel, et al., 2006). These unwanted side-effects can be explained by the involvement of the STN in motor, associative and limbic behavior. Current spread to the associative area, which is located ventrolaterally, and to the limbic area in the most ventromedial tip of the nucleus is responsible for the psychiatric side effects (Hamani, et al., 2004, Parent and Hazrati, 1995, Temel, et al., 2005). Therefore, electrophysiological unit recordings are utilized to identify the STN and optimize electrode placement. Also local field potentials (LFPS) are often measured from the implanted DBS electrodes. The LFP shows pathologic B oscillatory activity $(12-30 \mathrm{~Hz})$ in the STN of PD patients. This pathologic increase in $\beta$ activity is mainly observed within the dorsolateral motor region of the STN (Kuhn, et al., 2005, Moran, et al., 2008, Trottenberg, et al., 2007). The LFP represents the summed postsynaptic potentials of a group of neurons (Buzsaki, 2004), therefore it can be considered as the input activity. In contrast, the unit activity is a measure of the output activity. In human, the cortex is classically connected to the STN via the indirect pathway, which not only passes through the striatum and globus pallidus externa to the STN (Parent and Hazrati, 1995), but also via a monosynaptic pathway (Brunenberg, et al., 2012). Previously, we have shown in human that motor cortex stimulation (MCS) evoked responses in the unit activity, which were not present outside the STN and differed spatially within the STN (Janssen, et al., 2012). Strafella et al. (Strafella, et al., 2004) had similar findings when measuring subthalamic unit activity during transcranial magnetic stimulation. Considering the different neuronal origin of the LFP, a more detailed study of the response in the LFP to MCS will provide more insight into the subthalamic input activity and the pathways involved (Magill, et al., 2004). We hypothesized that the LFP is specifically responsive to MCS in the dorsolateral region of the STN, as this is the area believed to be involved in motor function (Hamani, et al., 2004). Therefore, in this study we present the cortically evoked potentials in the LFP signal in the subthalamic region. As the LFP is believed to represent the neural input activity, it could provide an interesting tool for locating the STN motor area during stereotactic surgery. This potential use was studied by determining the temporal and spatial extent of the evoked LFPs. These results were also compared to the unit responses, which show a specific response to cortical stimulation in the dorsal STN (Janssen, et al., 2012). 


\section{Methods}

\section{Subjects}

Patients were enrolled based on the same criteria used for standard STN DBS. Five patients (ages 52-70 years) were included, but only the procedure and results of the last patient are described. The stimulation protocols used in the other patients did not result in an STN response due to saturation of the amplifier in the first two patients and suboptimal MCS protocols in the remaining two patients. The study, including five patients, was approved by the Medical Ethical Committee of the Maastricht University Medical Centre and all the patients gave written informed consent.

The procedure has been previously described in detail by Janssen et al. (Janssen, et al., 2012). In short, subdural MCS with a strip of four electrodes (Model TS04R-SP10X-000; ADTech, Racine, WI, USA) was performed on the hand area of the motor cortex (stimulation settings: bipolar, monophasic, 0.2 ms, 7 or 15 mA, 200 stimuli). Concurrently, neuronal activity in and around the STN was measured using five microelectrodes (MicroMacroElectrode; InoMed, Emmendingen, Germany). Only local anesthesia was used. The stimulation amplitudes were determined based on the amplitude needed to obtain a motor evoked potential (MEP, $7 \mathrm{~mA}$ ).

In order to obtain LFPs from the raw signals, the signals were filtered using a non-causal second order band pass Butterworth filter between 3 and $95 \mathrm{~Hz} ; 50 \mathrm{~Hz}$ noise was removed using a notch filter. Subsequently, the signals were divided into epochs from 100 ms before stimulation until 200 ms after stimulation. All epochs belonging to the same location and resulting from the same stimulation settings were averaged. Significant deflections in the average LFPs were determined when five successive samples exceeded a threshold of plus or minus two times the standard deviation of the signal measured during $15 \mathrm{~mA}$ stimulation. LFP responses were compared to the responses in the unit activity. The unit responses were evaluated by peri-stimulus time histograms (PSTHs) in which significant changes were found by the change point analysis. A detailed description of the analysis of the unit activity is previously described (Janssen, et al., 2012). 


\section{Results}

LFP recordings in the anterior and lateral trajectories were made from 1.5 and $0.5 \mathrm{~mm}$ above the target until 1 and $2.5 \mathrm{~mm}$ below the target. These trajectories were inside the STN from $2 \mathrm{~mm}$ above the target until $2.5 \mathrm{~mm}$ below the target. Fig. 1 shows the LFPs and peri-stimulus time histograms (PSTHs) constructed using the responses in the unit activity (Janssen, et al., 2012) after cortical stimulation. The LFPs show a positive deflection around $43 \pm 3 \mathrm{~ms}$. This peak is present at all heights in the lateral trajectory and at -1.5 and $-0.5 \mathrm{~mm}$ in the anterior trajectory. Subsequently, negative peaks are present at $78 \mathrm{~ms}$ in the anterior trajectory and at $81 \mathrm{~ms}$ in the lateral trajectory at a height of $-1.5 \mathrm{~mm}$. At $-0.5 \mathrm{~mm}$ above the calculated target, this negative peak has disappeared. At $+1 \mathrm{~mm}$ in the anterior and lateral trajectory and at $+2.5 \mathrm{~mm}$ in the lateral trajectory, a positive peak is seen at $\sim 75 \mathrm{~ms}$ after stimulation. Finally, a significant negative peak is visible in the anterior trajectory at $+2.5 \mathrm{~mm}$. In the central and medial trajectory, the LFP response did show some significant peaks, but no specific pattern was visible. The LFP results did not correspond with the changes in the PSTH, which showed little to no response to stimulation (Janssen, et al., 2012).

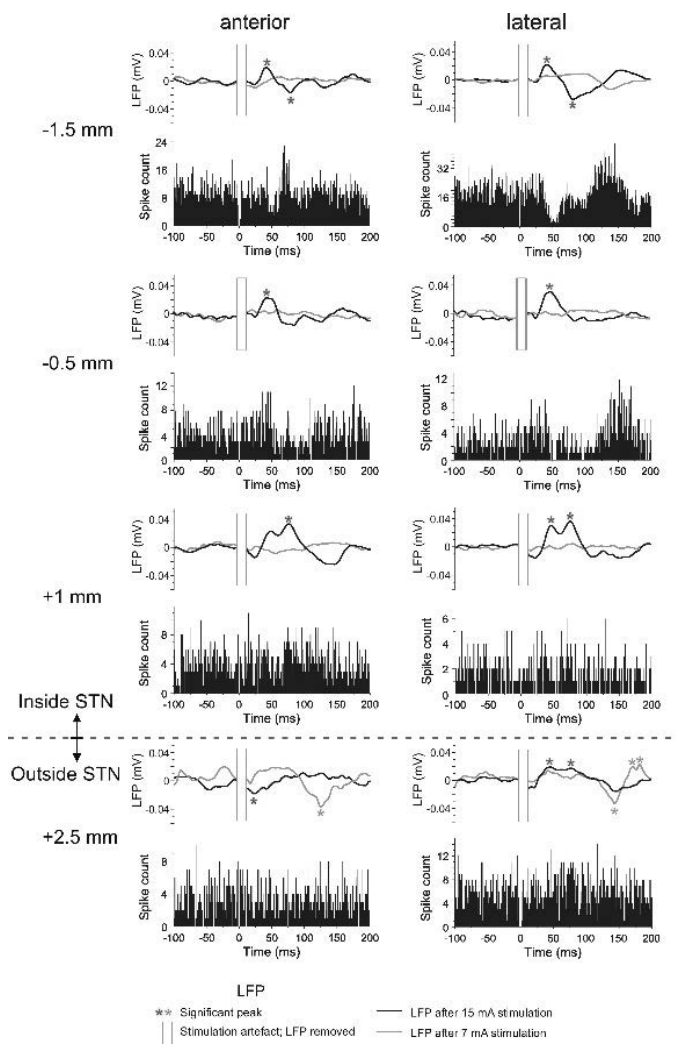

Fig. 1. The cortically evoked LFPs using a stimulation amplitude of 7 and $15 \mathrm{~mA}$ are plotted as well as the PSTHs of the anterior and lateral electrode starting $0.5 \mathrm{~mm}$ after the electrode first enters the STN $(1.5 \mathrm{~mm}$ above target) until it leaves the STN $(2.5 \mathrm{~mm}$ below target). The PSTHs using $7 \mathrm{~mA}$ stimulation did not show any significant responses, therefore only the PSTHs obtained with $15 \mathrm{~mA}$ are plotted. LFP: LFPs were averaged over all trials. An asterisk indicates a significant LFP peak, which is determined by exceeding a threshold of \pm 2 times the standard deviation from the signal during $15 \mathrm{~mA}$ stimulation. The PSTHs are partially adapted from Janssen et al. (2012). 
Responses were only visible in the LFPs when $15 \mathrm{~mA}$ stimulation was applied, but not when a stimulus amplitude of $7 \mathrm{~mA}$ was used; except for the responses shown at +2.5 $\mathrm{mm}$. This was in agreement with the fact that no significant responses to MCS were visible in the PSTHs while using an amplitude of 7 mA for MCS (Janssen, et al., 2012).

The positive peak at $43 \mathrm{~ms}$ corresponds with the start of the first inhibitory period found in the PSTHs at heights -1.5 and $-0.5 \mathrm{~mm}$. The negative peaks at 78 and $81 \mathrm{~ms}$ at a height of $-1.5 \mathrm{~mm}$ in the anterior and lateral trajectory are within the period of increased firing rate in the PSTHs from about 63-100 ms after stimulation. The positive peaks in the anterior and lateral trajectories at $\sim 75 \mathrm{~ms}$ are not seen in the PSTHs at these levels.

\section{Discussion}

In this study, for the first time evoked LFPs in the STN region following MCS in a PD patient have been described. We showed that evoked LFPs follow a specific pattern in the dorsal STN, namely first a positive deflection around $43 \mathrm{~ms}$ followed by a negative deflection around $80 \mathrm{~ms}$. The positive deflection is seen in the entire STN, but the negative deflection seems specific to the dorsolateral STN region. Some of the evoked LFP peaks are temporally and spatially linked to the unit responses to MCS.

We showed that the cortical input to the human STN can be visualized in the LFP. However, the temporal response in the LFP is not as clear-cut as in the rodent, although the LFP was averaged over many stimuli, which was not necessary in rodents (Magill, et al., 2004). In contrast to the animal data, the deflections in the LFP caused by the monosynaptic cortico-subthalamic pathway and the indirect cortico-striato-pallidosubthalamic pathway were not found. This could be due to difference in the size of the dendritic fields between species and a prominent lower cell density in the human compared to the rodent STN (Hammond and Yelnik, 1983) and (Marani, et al., 2008). Nonetheless, a clear positive deflection around $43 \mathrm{~ms}$ and a negative deflection around 80 ms were observed. The positive deflection was seen through the full ventro-dorsal axis of the STN and has a similar latency as observed in the rodents, which is the start of the long-lasting inhibitory period that may be caused by cortical disfacilitation (Magill, et al., 2004). The negative deflection, which was only observed in the dorsal region of the STN, can be explained as a sensor response caused by muscular contraction induced by MCS (Hanajima, et al., 2004). Movement related neuronal activity of the STN has earlier been described (Abosch, et al., 2002) and (Hutchison, et al., 1998).

Magill et al. (2004) showed that a negative deflection in the LFP (input) coincides with an excitation shown in the PSTH (output) and vice versa. We clearly see that the positive peaks in the averaged LFP coincided with the long lasting inhibitory periods 
found in the PSTH. This positive LFP peak was also present at a height of $+1 \mathrm{~mm}$, at which the inhibition was no longer present in the PSTH. In this PD patient, the LFPs, thus, extended to a broader area than the unit responses. There can be different reasons for this. First, it should be considered that the LFP reflects the summed postsynaptic potentials of a group of neurons (Buzsaki, 2004), while multi-unit activity reflects the action potentials measured in one or just a few neurons. Changes in the synaptic activity do not always lead to an action potential. Furthermore, LFPs exhibit strong low-pass filtering properties (Bedard, et al., 2006), which could account for a larger responsive region of the low frequency LFP signal as compared to the high frequency spike signal. It has been argued that LFPs are volume conducted over a radius of $0.25 \mathrm{~mm}$ (Katzner, et al., 2009). As the measurement at $+2.5 \mathrm{~mm}$ was just outside of the STN (+2 mm was still inside of the STN), it might be that the LFPs originating from STN activity were volume conducted outside the STN. The negative peak in the LFPs at a height of $-1.5 \mathrm{~mm}$ around 80 ms coincided with an increased firing rate shown in the PSTHs. However, the remaining PSTH was not reflected in the LFP. Additionally, we found significant deflections in the LFPs when no significant changes in the PSTH were found (Fig. 1: $+2.5 \mathrm{~mm}$ ). These were probably caused by a low signal to noise ratio outside the STN, since also at $7 \mathrm{~mA}$ significant peaks were found.

Finally, we only observed a response after MCS while using a stimulation amplitude of $15 \mathrm{~mA}$, but not after $7 \mathrm{~mA}$ stimulation. Moreover, in the LFP no excitatory monosynaptic response could be observed. This is not in line with what we expected, as our modeling study shows activation of pyramidal axons at $7 \mathrm{~mA}$ (Zwartjes, et al., 2012). A reason for this discrepancy could be suboptimal placement of the stimulation electrode or it could be that the synaptic strength from the cortical afferents is less strong than thus far assumed. To our knowledge no quantitative studies exist on the number of synapses in the STN with a cortical origin. This would imply that a STN response is only present when a high number of pyramidal neurons is activated. On the other hand a strong coherence is present between the prefrontal cortex and the STN, which implicates a strong cortico-subthalamic connectivity (Litvak, et al., 2011). Furthermore, the duration of the stimulation artifact overlapped with the expected timing of the monosynaptic response, which could have made this response invisible. We believe, despite the small sample size, that our results are important from a clinical perspective. In literature, it is being debated whether additional invasive procedures are warranted to improve the quality of the DBS procedure (Alegre, et al., 2012). We believe that neurophysiological recordings can be helpful to increase the accuracy of the implantation of DBS electrodes. Accurate targeting is the limiting step in achieving maximal benefit on motor symptoms and minimizing side effects on behavior and cognition. Here we showed that the evoked LFPs were not restricted to a certain area of the STN and also extended beyond STN borders. Thus, the evoked LFPs did not have a spatial resolution 
high enough to locate the STN motor area. Further adaptation of the stimulation and recording protocol will decrease surgery time and will be of additional value to the standard used intra-operative tests to define the optimal site of implantation. To achieve this goal, computational models of cortical stimulation should be made to predict the optimal cortical stimulation site, electrode size and stimulation parameters (Zwartjes, et al., 2012). Moreover, since MCS had an unexpectedly high risk of inducing seizures (Janssen, et al., 2012), alternative non-invasive techniques, such as high resolution imaging, and other electrophysiological markers, such as the $ß$ activity, should be further investigated to explore their possibilities to target the motor part of the STN (Lambert, et al., 2012, Zaidel, et al., 2010). Imaging techniques can be combined with intra-operative electrophysiological information providing a more precise indication of which area of the STN should be stimulated. The next step then would be to develop DBS electrodes that are able to stimulate a selective area (Martens, et al., 2011). Combining the improved identification of the STN motor region and the development of DBS electrodes with a higher spatial resolution will optimize DBS therapy for PD patients.

\section{Acknowledgements}

The authors gratefully acknowledge the support of the BrainGain Smart Mix Programme of the Netherlands Ministry of Economic Affairs and the Netherlands Ministry of Education, Culture and Science (grant no.: SSM06011). 


\section{References}

1. Abosch, A., Hutchison, W. D., Saint-Cyr, J. A., Dostrovsky, J. O., and Lozano, A. M., 2002. Movementrelated neurons of the subthalamic nucleus in patients with Parkinson disease. J Neurosurg 97, 11671172.

2. Alegre, M., Hallett, M., Olanow, C. W., and Obeso, J. A., 2012. Technical advances in deep brain stimulation: How far is enough? Mov Disord 27, 341-342.

3. Bedard, C., Kroger, H., and Destexhe, A., 2006. Model of low-pass filtering of local field potentials in brain tissue. Physical review. E, Statistical, nonlinear, and soft matter physics 73, 051911.

4. Brunenberg, E. J., Moeskops, P., Backes, W. H., Pollo, C., Cammoun, L., Vilanova, A., Janssen, M. L., Visser-Vandewalle, V. E., Ter Haar Romeny, B. M., Thiran, J. P., and Platel, B., 2012. Structural and Resting State Functional Connectivity of the Subthalamic Nucleus: Identification of Motor STN Parts and the Hyperdirect Pathway. PLoS One 7, e39061.

5. Buzsaki, G., 2004. Large-scale recording of neuronal ensembles. Nat Neurosci 7, 446-451.

6. Deuschl, G., Schade-Brittinger, C., Krack, P., Volkmann, J., Schafer, H., Botzel, K., Daniels, C., Deutschlander, A., Dillmann, U., Eisner, W., Gruber, D., Hamel, W., Herzog, J., Hilker, R., Klebe, S., Kloss, M., Koy, J., Krause, M., Kupsch, A., Lorenz, D., Lorenzl, S., Mehdorn, H. M., Moringlane, J. R., Oertel, W., Pinsker, M. O., Reichmann, H., Reuss, A., Schneider, G. H., Schnitzler, A., Steude, U., Sturm, V., Timmermann, L., Tronnier, V., Trottenberg, T., Wojtecki, L., Wolf, E., Poewe, W., and Voges, J., 2006. A randomized trial of deep-brain stimulation for Parkinson's disease. N Engl J Med 355, 896-908.

7. Hamani, C., Saint-Cyr, J. A., Fraser, J., Kaplitt, M., and Lozano, A. M., 2004. The subthalamic nucleus in the context of movement disorders. Brain 127, 4-20.

8. Hammond, C., and Yelnik, J., 1983. Intracellular labelling of rat subthalamic neurones with horseradish peroxidase: computer analysis of dendrites and characterization of axon arborization. Neuroscience 8 , 781-790.

9. Hanajima, R., Dostrovsky, J. O., Lozano, A. M., Hutchison, W. D., Davis, K. D., Chen, R., and Ashby, P., 2004. Somatosensory evoked potentials (SEPs) recorded from deep brain stimulation (DBS) electrodes in the thalamus and subthalamic nucleus (STN). Clin Neurophysiol 115, 424-434.

10. Hutchison, W. D., Allan, R. J., Opitz, H., Levy, R., Dostrovsky, J. O., Lang, A. E., and Lozano, A. M., 1998. Neurophysiological identification of the subthalamic nucleus in surgery for Parkinson's disease. Ann Neurol 44, 622-628.

11. Janssen, M. L., Zwartjes, D. G., Temel, Y., van Kranen-Mastenbroek, V., Duits, A., Bour, L. J., Veltink, P. H., Heida, T., and Visser-Vandewalle, V., 2012. Subthalamic neuronal responses to cortical stimulation. Mov Disord 27, 435-438.

12. Katzner, S., Nauhaus, I., Benucci, A., Bonin, V., Ringach, D. L., and Carandini, M., 2009. Local origin of field potentials in visual cortex. Neuron 61, 35-41.

13. Kuhn, A. A., Trottenberg, T., Kivi, A., Kupsch, A., Schneider, G. H., and Brown, P., 2005. The relationship between local field potential and neuronal discharge in the subthalamic nucleus of patients with Parkinson's disease. Exp Neurol 194, 212-220.

14. Lambert, C., Zrinzo, L., Nagy, Z., Lutti, A., Hariz, M., Foltynie, T., Draganski, B., Ashburner, J., and Frackowiak, R., 2012. Confirmation of functional zones within the human subthalamic nucleus: Patterns of connectivity and sub-parcellation using diffusion weighted imaging. Neuroimage 60, 83-94.

15. Litvak, V., Jha, A., Eusebio, A., Oostenveld, R., Foltynie, T., Limousin, P., Zrinzo, L., Hariz, M. I., Friston, K., and Brown, P., 2011. Resting oscillatory cortico-subthalamic connectivity in patients with Parkinson's disease. Brain : a journal of neurology 134, 359-374.

16. Magill, P. J., Sharott, A., Bevan, M. D., Brown, P., and Bolam, J. P., 2004. Synchronous unit activity and local field potentials evoked in the subthalamic nucleus by cortical stimulation. J Neurophysiol 92, 700714.

17. Marani, E., Heida, T., Lakke, E. A., and Usunoff, K. G., 2008. The subthalamic nucleus. Part I: development, cytology, topography and connections. Adv Anat Embryol Cell Biol 198, 1-113, vii. 
18. Martens, H. C., Toader, E., Decre, M. M., Anderson, D. J., Vetter, R., Kipke, D. R., Baker, K. B., Johnson, M. D., and Vitek, J. L., 2011. Spatial steering of deep brain stimulation volumes using a novel lead design. Clinical neurophysiology : official journal of the International Federation of Clinical Neurophysiology 122, 558-566.

19. Moran, A., Bergman, H., Israel, Z., and Bar-Gad, I., 2008. Subthalamic nucleus functional organization revealed by parkinsonian neuronal oscillations and synchrony. Brain 131, 3395-3409.

20. Parent, A., and Hazrati, L. N., 1995. Functional anatomy of the basal ganglia. I. The cortico-basal gangliathalamo-cortical loop. Brain Res Brain Res Rev 20, 91-127.

21. Strafella, A. P., Vanderwerf, Y., and Sadikot, A. F., 2004. Transcranial magnetic stimulation of the human motor cortex influences the neuronal activity of subthalamic nucleus. Eur J Neurosci 20, 2245-2249.

22. Temel, Y., Blokland, A., Steinbusch, H. W., and Visser-Vandewalle, V., 2005. The functional role of the subthalamic nucleus in cognitive and limbic circuits. Prog Neurobiol 76, 393-413.

23. Temel, Y., Kessels, A., Tan, S., Topdag, A., Boon, P., and Visser-Vandewalle, V., 2006. Behavioural changes after bilateral subthalamic stimulation in advanced Parkinson disease: a systematic review. Parkinsonism Relat Disord 12, 265-272.

24. Trottenberg, T., Kupsch, A., Schneider, G. H., Brown, P., and Kuhn, A. A., 2007. Frequency-dependent distribution of local field potential activity within the subthalamic nucleus in Parkinson's disease. Exp Neurol 205, 287-291.

25. Zaidel, A., Spivak, A., Grieb, B., Bergman, H., and Israel, Z., 2010. Subthalamic span of beta oscillations predicts deep brain stimulation efficacy for patients with Parkinson's disease. Brain 133, 2007-2021.

26. Zwartjes, D. G., Heida, T., Feirabend, H. K., Janssen, M. L., Visser-Vandewalle, V., Martens, H. C., and Veltink, P. H., 2012. Motor cortex stimulation for Parkinson's disease: a modelling study. J Neural Eng 9, 056005. 


\title{
CHAPTER 8
}

\author{
Automated gait analysis in \\ bilateral Parkinsonian rats and \\ the role of L-DOPA therapy
}

Westin JE, Janssen MLF, Sager TN, Temel Y

Behavioural Brain Research 2012;226 : 519- 528. 


\section{Abstract}

Gait disturbances and postural instability represent major sources of morbidity in Parkinson's disease (PD), and respond poorly to current treatment options. Some aspects of gait disturbances can be observed in rodent models of PD; however, knowledge regarding the stability of rodent gait patterns over timeis lacking. Here we investigated the temporal constancy and reproducibility of gait patterns in neurologically intact and bilaterally 6-hydroxydopamine (6-OHDA) lesioned rats, by using an automated quantitative gait analysis method (CatWalk). The bilateral neurotoxin injections into the medial forebrain bundle resulted in an average dopamine (DA) loss of 70\% in striatum, which corresponds to the DA levels observed in moderate-mid stage human PD. Rats were tested weekly during one month, and we found that in intact rats all parameters investigated remained constant over multiple tests. The 6-OHDA lesioned rats were impaired in several aspects of gait, such as stride length, swing speed, stance duration, step cycle duration, and base of support. However the stance and step cycle deficits were transient, the performance of 6-OHDA lesioned rats were indistinguishable from control rats by the last test session with regard to these parameters. Finally, we found that administration of a single dose of levodopa (I-DOPA) to the 6-OHDA lesioned rats could counteract all but one observed deficits. Based on these findings we conclude that the gait pattern of intact rats is highly reproducible, 6-OHDA lesioned rats display impairments in gait, and I-DOPA can counteract most deficits seen in this model of experimental PD. 


\section{Introduction}

Parkinson's disease is a progressive neurodegenerative disorder characterized by degeneration of the midbrain dopaminergic neurons of the substantia nigra pars compacta (SNc; (Dauer and Przedborski, 2003)). Nigral degeneration is recognized as a main cause of the key motor symptoms in PD such as akinesia, rigidity, and tremor (Gelb, et al., 1999); however, degeneration of other brain areas such as the cholinergic mesencephalic motor region, the raphe nuclei and the noradrenergic locus coeruleus (LC) contribute to the symptomatology (Devos, et al., 2010, Karachi, et al., 2010, Tan, et al., 2011, Zarow, et al., 2003). The classical concept is that the first motor symptoms of PD usually appear in patients when approximately $60 \%$ of the SNc dopamine (DA) neurons have degenerated and $70-80 \%$ of the normal striatal DA level is lost (Hornykiewicz, 1975, Marsden, 1990). The majority of the key symptoms of PD can be treated with medication (Yuan, et al., 2010), and in later stages by deep brain stimulation (Williams, et al., 2010). Other important, debilitating and clinically relevant symptoms are gait and balance disturbances, which partially respond poorly to the classical treatments (Browner and Giladi, 2010, Devos, et al., 2010, Pullman, et al., 1988). These axial symptoms are characterized by hesitation of gait initiation and a reduction of kinematic gait parameters such as stride length, gait speed and swing phase duration in moderate-mid stage PD (Blin, et al., 1990, Devos, et al., 2010, Morris, et al., 1994). Balance is typically preserved in the early disease stages, and falls are rare during the first years after disease onset (Wenning, et al., 1999). Falls, freezing, festination, and postural instability become more pronounced in late-stage PD (Kemoun and Defebvre, 2001). Several rodent models that mimicks various motor symptoms of PD have been developed, such as unilateral 6- hydroxydopamine (6-OHDA) lesions in rats, 1-methyl-4-phenyl- 1,2,3,6tetrahydropyridin (MPTP) lesions in mice, as well as transgenic mice models (Cenci and Ohlin, 2009, Meredith, et al., 2008, Taylor, et al., 2010). To date numerous behavioural tests have been applied to these models in order to investigate different aspects of gait. In animals with a unilateral 6-OHDA lesion, the cylinder test is commonly used to score asymmetries in forelimb use or akinesia (Cenci, et al., 2002, Shi, et al., 2004, Tillerson, et al., 2001). The test is limited to forelimb movements, manually scored, and repeated testing can leadto habituation problems. Motor performance tests like rotarod and treadmill are based on forced locomotion, and typically investigate the maximum performance time at a set speed (Carter, et al., 1999, Chang, et al., 2003, Mazarakis, et al., 2005, Shi, et al., 2006). These tests do not dissect specific errors in walking patterns that result in falls/altered position; however, an ambitious video monitoring system of treadmill walking developed by Amende et al. (Amende, et al., 2005) allows a more indepth analysis of dynamic gait parameters. Other tests that focus more on the balance aspect of walking are the beam walking and the ladder-rung test (Carter, et al., 1999, 
Fleming, et al., 2004, Klein, et al., 2009, Metz and Whishaw, 2002), where animals are manually scored for the number of foot falls during the walk and the latency to cross. If scored by the thorough scoring schemes developed (Metz and Whishaw, 2009), the tests are sensitive to subtle dopamine-related deficits, but do only provide insight on a few parameters of gait. Static gait parameters are often investigated by footprint analysis, where animal walks down a strip of paper after the front or hind paws are dipped in ink (de Medinaceli, et al., 1982, Klein, et al., 2009, Metz, et al., 2005). The introduction of the CatWalk automated gait analysis method has enabled simultaneous objective quantification of a large number of both static and dynamic gait parameters during voluntary walking (Hamers, et al., 2001). The method has been applied to evaluate walking patterns in models of pain (Gabriel, et al., 2007, Gabriel, et al., 2009), spinal cord injuries (Deumens, et al., 2007, Gensel, et al., 2006, Hamers, et al., 2006, Koopmans, et al., 2005), Huntington's disease (Vandeputte, et al., 2010), and Parkinson's disease (Chuang, et al., 2010, Vandeputte, et al., 2010, Vlamings, et al., 2007). The majority of the studies have focused on comparing one or two test sessions on the CatWalk, often before and after an intervention. The aim of this study was to investigate the constancy and reproducibility of gait parameters, in the same animals, during multiple test sessions in neurologically intact and bilaterally 6-OHDA-lesioned rats, where the toxin was injected into the medial forebrain bundle (mfb). In addition, we treated the 6-OHDA rats with I-DOPA to evaluate which gait changes were responsive to DA therapy.

\section{Material and Methods}

\section{Subjects}

Male Lister-Hooded rats (Charles River, Germany: weight $275 \mathrm{~g}$ at the beginning of the experiment) were housed in pairs under a 12-h reversed light/dark cycle (light on from $6 \mathrm{pm}$ to $6 \mathrm{am})$, with ad libitum access to food and water. Room temperature, $(21 \pm 2 \circ \mathrm{C})$ humidity $(60 \pm 10 \%)$ and air exchange (16 times per hour) were automatically controlled. Experiments were carried out in accordance with the ethical rules of the Danish National Committee for use of Laboratory Animals.

\section{Surgery}

Rats were anesthetized with $2 \mathrm{ml} / \mathrm{kg}$ Hypnorm/Dormicum (fentanyl citrate, 0.079 $\mathrm{mg} / \mathrm{ml}$; fluanisone, $2.5 \mathrm{mg} / \mathrm{ml}$; midazolam, $1.25 \mathrm{mg} / \mathrm{ml}$; Hoffman-LaRoche Basel, Switzerland) before subjected to surgery. Rats were placed in a stereotactic frame (Kopf Instruments, model 900; CA, USA) and received one injection of 6-OHDA per hemisphere in the $\mathrm{mfb}$ at the following coordinates relative to Bregma (in $\mathrm{mm}$ ); tooth bar -3.2, AP -2.2, ML \pm 1.5 , DV $-8.0 \mathrm{~mm}$ (from the dura mater) (Paxinos and Watson, 1998). 
6-OHDA was dissolved in $0.02 \%$ ascorbic acid at a concentration of $2.5 \mu \mathrm{g}$ free base $/ \mu \mathrm{l}$ and kept dark. $2 \mu \mathrm{l}$ ( $5 \mu \mathrm{g}$ free base) per hemisphere was continuously infused over 2 min. Twenty rats were subjected to bilateral 6-OHDA infusion, a group of six rats received bilateral infusion of ascorbic acid (sham operated), and an additional six rats were only sedated with Hypnorm/Dormicum (normal control group).

All the animals were given Temgesic (Reckitt Benckiser, Berkshire, UK) in conjunction with the surgery/anesthesia and a daily injection of Rimadyl (Carprofen; Pfizer Animal Health, New York, USA) for 5 consecutive days after surgery to reduce postoperative pain. All rats received supplementary wet food and fruit the first week post surgery, and supplementary sucrose (5\%) in drinking water, to control the weight loss. Rats that lost more than $20 \%$ of their pre-operative weight were immediately taken out of the study and euthanized $(n=3)$. One sham rat was lost after surgery.

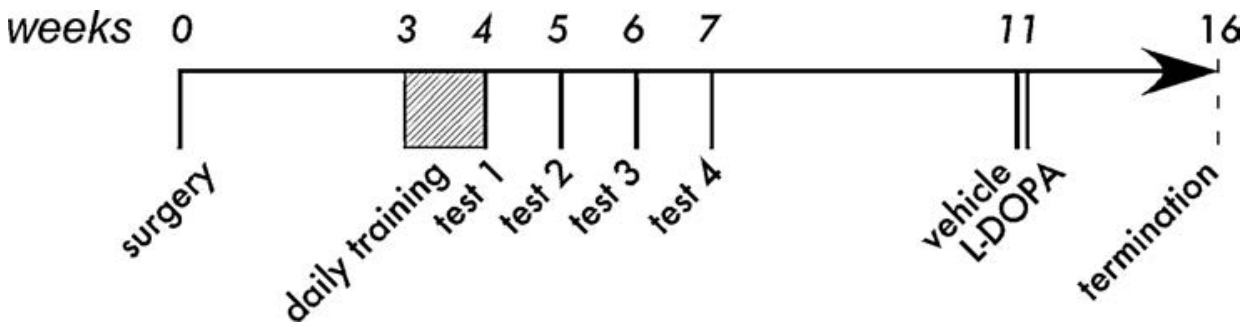

Fig. 1. Schematic illustration of the experimental design.

\section{Experimental groups}

These procedures resulted in three experimental groups consisting of control $(n=6)$, sham-operated $(n=5)$, bilateral 6-OHDA lesioned $(n=14)$ animals. All groups where tested in the CatWalk apparatus. Three bilateral lesioned rats were taken out of the study prior to CatWalk training as their weight loss was less than $10 \%$. Previous work in our laboratory has shown a strong correlation between weight loss and the severity of SNc dopaminergic cell loss, and in rats that lost more than $10 \%$ of the pre-operative weight, striatal DA levels were typically reduced by at least $70 \%$ on average of both hemispheres (Lundbeck, unpublished data).

\section{Automated gait analysis and I-DOPA administration}

The CatWalk (Noldus TM) is a computer-assisted automated quantitative gait analysis system that allows rapid and objective quantification of multiple static and dynamic gait parameters. It consists of a glass walkway floor where light form a fluorescent tube is internally reflected, with a digital camera underneath. When a paw is placed on the glass, the light escapes the walkway, which results in illumination of the exact area of contact. The image is captured by the camera, digitized, and stored on a computer for off-line analysis. 
Rats were trained to walk down the runway daily one week prior to the first test session (Koopmans, et al., 2005). One test session consisted of three consecutive uninterrupted runs per rat, and a mean value per parameter was used in the statistical analysis. The first test session was performed 4 weeks post surgery, and subsequently rats where tested once a week during four weeks. A schematic overview of the experimental time lines is depicted in Fig. 1.

As it turned out that it was difficult to get the bilaterally 6-OHDA lesioned rats to cross the CatWalk when compared to the controls, we had to slightly modified our inclusion criteria so that each rat had to perform at least two runs per test session, and an acceptable run was defined as an uninterrupted walk of at least two-third of the runway. All test sessions took place between 8 am and 3 pm, during the animals' dark cycle.

One month after completing all four tests, the effect of I-DOPA on gait was investigated in a subset of the 6-OHDA rats. After base-line measurements, all rats were tested 30-60 min after an s.c. injection of $0.9 \% \mathrm{NaCl}$ (vehicle). On the next day, control and sham operated rats were tested with vehicle again, whereas rats with bilateral 6-OHDA treatment were administered $6 \mathrm{mg} / \mathrm{kg}$ I-DOPA methylester (Sigma-Aldrich Denmark $\mathrm{A} / \mathrm{S}$, Copenhagen, Denmark) mixed with $15 \mathrm{mg} / \mathrm{kg}$ peripheral dopadecarboxylase inhibitor Benserazide (Sigma-Aldrich) 30 min prior to the first run.

Below, we present a description of the parameters that were examined in the present study. For a complete description of the CatWalk system, see Refs. (Hamers, et al., 2006, Hamers, et al., 2001, Vlamings, et al., 2007).

\section{Static paw parameters}

Base of support (BOS): the average width $(\mathrm{mm})$ between either the front paws or the hind paws; stride length: the distance $(\mathrm{mm})$ between successive placements of the same paw; relative paw position: the distance between the position of the hind paw and the position of the previously placed ipsilateral front paw, in the same step cycle. If the hind paw is placed (partially) after the forepaw, the distance is positive, otherwise negative.

\section{Dynamic paw parameters}

Stance duration: the duration (s) of contact of a paw with the runway glass plate; swing duration: the duration ( $s$ ) of no contact of a paw with the glass plate; step cycle: the time in seconds between two consecutive contacts with the glass plate of the same paw (i.e. stance + swing phase); duty cycle: express stance phase as a percentage of the step cycle. 


\section{Speed and coordination parameters}

Swing speed: the speed $(\mathrm{m} / \mathrm{s})$ of the paw during swing phase. Regularity index: a measure of interlimb coordination expressed as the number of normal step sequence patterns relative to the total number of paw placements. There are six possible sequence patterns, and a RI of $100 \%$ indicates that each paw was correctly placed during the walkway crossing.

\section{Tissue preparation}

Rats were decapitated 10 days after the last test session, the right and left striatum (22$28 \mathrm{mg}$ ) were rapidly dissected, frozen, and stored at $-80 \circ \mathrm{C}$ for further HPLC processing. The caudal part of the brain was immersed in ice cold paraformaldehyde (4\%; $\mathrm{pH} 7.4$ ) for $48 \mathrm{~h}$, transferred to $20 \%$ sucrose for $24 \mathrm{~h}$ and thereafter sectioned coronally on a freezing microtome at $30 \mu \mathrm{m}$ thickness.

\section{HPLC procedures}

The level of DA and noradrenalin (NA) in the striatum were measured using high pressure liquid chromatography (HPLC) with electrochemical detection. The tissue was homogenised with a cell disrupter (Ultra-Turrax, IKA-Werke GmbH, Germany) in $1 \mathrm{ml}$ icecold $0.05 \mathrm{M}$ perchloric acid. The homogenate was then centrifuged at $20,000 \times \mathrm{g}$ for 15 $\min$ at $4{ }^{\circ} \mathrm{C}$, and the supernatant was filtered through a Millipore filter (pore size, 0.22 $\mu \mathrm{m})$. Monoamines were separated by reverse-phase liquid chromatography (ODS 150 $\times 2 \mathrm{~mm}$ column). For DA, the mobile phase consisted of $150 \mathrm{mM} \mathrm{NaH2PO4,} 4.8 \mathrm{mM}$ citric acid monohydrate, $3 \mathrm{mM}$ dodecyl sulphate, $50 \mu \mathrm{M}$ EDTA, 11. 3\% methanol, and $16.7 \%$ acetonitrile $(\mathrm{pH} \mathrm{5.6)}$ at a flow rate of $0.2 \mathrm{ml} / \mathrm{min}$. Electrochemical detection was accomplished using a coulometric detector with the potential set to E2 $=200 \mathrm{mV}$ (Coulochem, II, USA). For NA, the mobile phase consisted of $75 \mathrm{mM}$ lithium acetate, $4 \mathrm{mM}$ sodium 1-heptanesulfonic acid, $100 \mu \mathrm{M}$ EDTA, and 7\% methanol $(\mathrm{pH} 4.7)$ at a flow rate of $0.25 \mathrm{ml} / \mathrm{min}$. Electrochemical detection was accomplished using a coulometric detector (Coulochem, II, USA) set to the following potentials; guard cell (350 mV), E1/E2 $(-75 / 150 \mathrm{mV})$.

\section{TH immunohistochemistry}

Besides HPLC measurement of DA, we performed cell counts to study the potential relation of the concentration of DA and the number of dopaminergic cells in the SNc. For this purpose we stained the SNc for tyrosine hydroxylase (TH). TH immunohistochemistry was carried out using mouse anti-TH (diluted 1:100, kindly supplied by Dr. C. Cuello, Montreal, Canada) as primary antibody. After rinsing with TBS-Triton X-100 (TBS-T), Tris-buffered solution (TBS) and TBS-T, tissue was incubation with the secondary antibody (diluted 1:400 donkey anti-mouse biotin; Jackson Immunoresearch Laboratories, West Grove, 
USA). After rinsing, sections were incubated with ABC-kit (diluted 1:800, Vector laboratories, USA). To visualize the horseradish peroxide (HRP) reaction product, sections were incubated with 3,3'-diaminobenzidine tetrahydrochloride (DAB) (Temel, et al., 2005).

\section{Stereological cell counting}

Stereological analysis was performed using a stereology workstation (CASTGRID- Computer Assisted Stereological Toolbox, Olympus, Denmark). After exactly tracing the boundaries of the left and right SNc on microscopic video images displayed on a monitor (magnification 10x), numbers of tyrosine hydroxylase immunoreactive (THir) neurons and total numbers of neurons were evaluated with the Optical Fractionator (magnification 40x) (Schmitz and Hof, 2005). Only neurons that came into focus within unbiased virtual counting spaces distributed in a systematic-random fashion throughout the delineated regions were counted. Estimated numbers of neurons were calculated from the numbers of counted neurons and the corresponding sampling probability.

\section{Statistical analysis}

All individual runway crossings were analyzed using the CatWalk software. For each CatWalk parameter the rat's individual averages were calculated over two or, if available, three runs and used to obtain group means and standard errors (mean + S.E.M.). For parameters were each paw's value was given separately, primary analysis revealed that the two front paws always gave almost identical numerical output for parameters investigated in the present study, and the same held true for the hind paws. No significant differences between left and right paws were found with a 2-way analysis of variance (ANOVA) test. Thus, averages for front and hind paw parameters, respectively, were calculated and used in the statistical analyses.

All data sets were tested for normality distribution with the Kolmogorov-Smirnov $(\mathrm{K}-\mathrm{S})$ test prior to any statistical analyses. Statistical comparisons were performed using the general linear model with variables group and run, repeated measures analysis (2WAY RM ANOVA; repeated factor: run). Front paws were analyzed separately from hind paws when applicable (all parameters except print position and regularity index), as most statistical software cannot calculate 3-way repeated measures ANOVA. The IDOPA experiment was analyzed separately, using 2-way ANOVA (factors group and front/hind paw). CatWalk data were analyzed by statistical software SigmaStat 3.0. To evaluate significant differences in more detail, pair-wise multiple comparisons with post hoc Student-Neuman-Keul's test was used.

The total level of DA in the left and right striatum and the number of THir neurons in the left and right SNc and the striatal DA levels were averaged per animal. The quantitative data on the total number of THir cells in the SNc were analyzed using independent samples T-test. The correlation between THir cell count and DA levels in the stria- 
tum was analyzed using a Pearson's correlation. Data were analyzed using SPSS statistical software (version 15.0). A value of $p<0.05$ was considered significant.

\section{Results}

\section{Bilateral mfb lesions}

The survival rate after the mfb 6-OHDA lesions was $85 \%$ ten days after the injections. At the time of the first test session there was some non-significant variation in the mean weight between the groups, and the difference persisted throughout the experiment. Two rats in the bilateral 6-OHDA group were removed before the data analysis since they failed to meet the CatWalk inclusion criteria. The final number of animals included in the data analyses were: control $n=6$; sham $n=5$; and bilateral 6-OHDA $n=12$.

\section{Striatal monoamine levels and SNc cell count}

The average striatal level of DA was reduced by $70.2 \pm 5.1 \%$ in the group that received bilateral injections of 6-OHDA ( $p<0.001$ vs. control and sham; Fig. 2A). The bilateral 6 OHDA group had an average reduction of striatal NA with $69.5 \pm 4.4 \%$ ( $p<0.001$ vs. control and sham; Fig. 2B). The SNc THir cell count (average left and right) was reduced by $51 \pm 8.8 \%$ ( $p<0.05$ vs. control and sham) in the groups that received bilateral injection of 6-OHDA (Fig. 2C) A significant correlation was found between the striatal DA levels and the SNc THir cell count (R2 = 0.61, $p=0.002$; Fig. 2D).

\section{CatWalk parameters in normal and bilaterally 6-OHDA lesioned rats}

All data sets except the swing duration passed the K-S normality test. The swing data was moderately positively skewed, but the skewness was not pronounced enough to cause a skewed distribution of the swing dependent step and duty cycle data sets. All data sets passed the equal variance test. The main findings are schematically summarized in Table 1.

Table 1. Catwalk parameters in 6-OHDA animals vs. control groups.

\begin{tabular}{lccc}
\hline & Forelimb & Hindlimb & General \\
\hline Base of support & $\downarrow$ & - & \\
Stride length & $\downarrow$ & $\downarrow$ & \\
Swing speed & $\downarrow$ & $\downarrow$ & - \\
Regularity index & & $\uparrow \sim$ & - \\
Relative paw position & $\uparrow \sim$ & - & \\
Stance phase & $-\sim$ & & $\uparrow$ \\
Swing phase & & & \\
Step cycle & & & \\
\hline
\end{tabular}

$\downarrow$ : Significant decrease, $\uparrow$ : significant increase, - : no significant change, $\sim$ : not stable over time. 

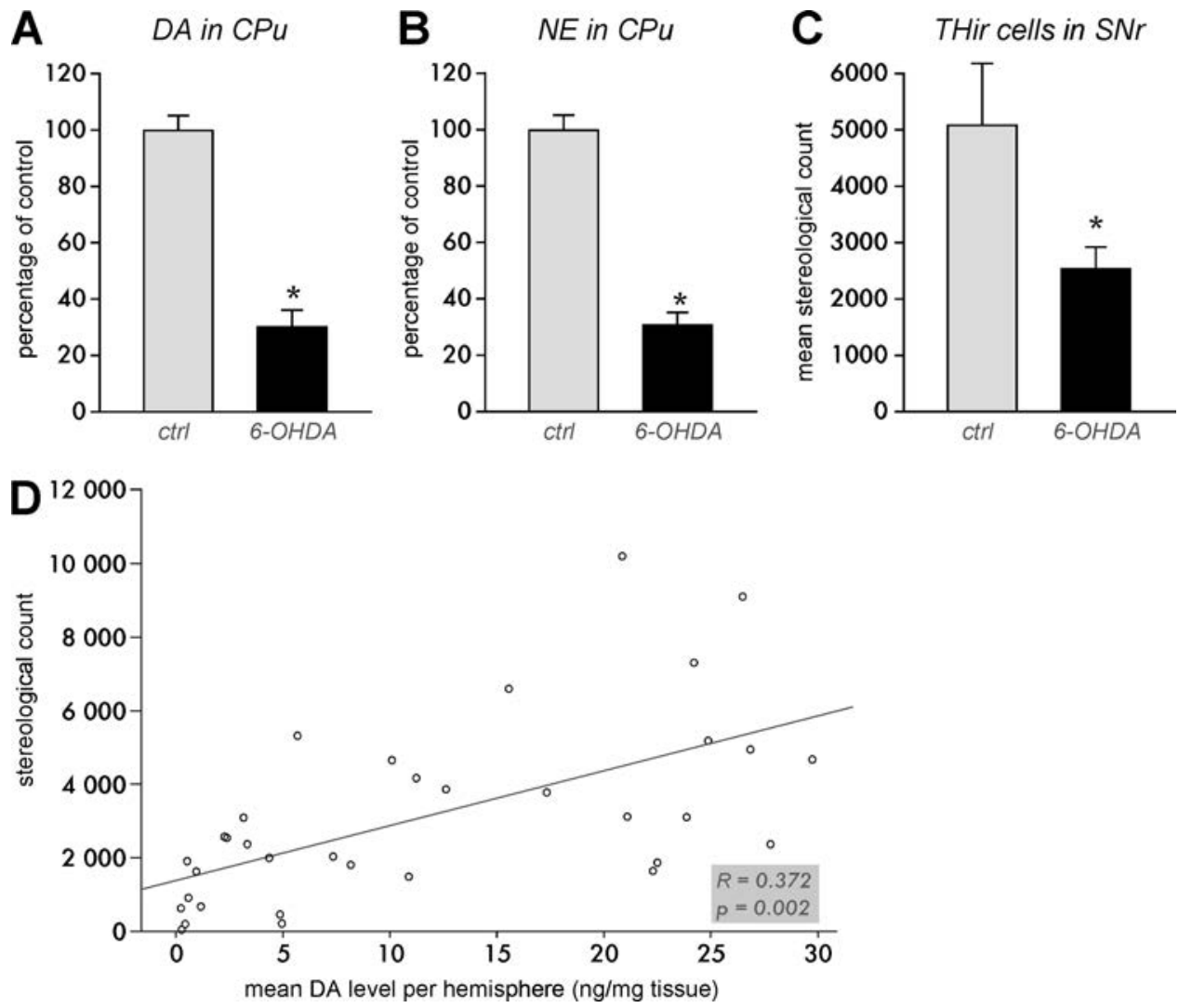

Fig. 2. Reduction of striatal monoamines and nigral DA neurons in bilaterally 6-OHDA lesioned rats. Striatal dopamine (A) and noradrenalin (B) levels were reduced in 6-OHDA rats, as was the number of nigral DA neurons (C). ${ }^{*} p<0.005$ compared to sham-lesioned rats. The number of nigral dopamine neurons is positively correlated to striatal dopamine levels (D).

Base of support

The forelimb base of support was significantly smaller in the bilaterally lesioned 6-OHDA rats compared to control rats $(p=0.01)$, and compared to sham operated animals $(p=$ 0.033). Hind limb BOS did not differ between the groups (Fig. 3A).

Stride length

The stride length was similar between front and hind paws in all groups (Fig. 3B), but the bilateral 6-OHDA rats took significantly shorter steps than control and sham operated rats $\left(p_{\text {front }}=0.002\right.$ and $p_{\text {hind }}<0.001$ vs. control; $p_{\text {front }}=0.005$ and $p_{\text {hind }}=0.003$ vs . sham). 


\section{Swing speed}

Swing speed was decreased in the bilateral 6-OHDA-group for both forelimbs $(p<0.001$ vs. control; $p=0.002$ vs. sham) and hind limbs ( $p<0.001$ vs. control and sham; Fig. $3 C$ ).

\section{Regularity index}

The overall ANOVA for regularity index (interlimb coordination) was significant at the level of group (control: $96.18 \pm 0.88 \%$; sham: $96.68 \pm 0.91 \%$; bilateral 6-OHDA: $93.64 \pm$ $0.73 \% ; p=0.047)$, but no significant differences were detected with post hoc test.

\section{Relative paw position}

No significant differences between either group or side (left vs. right) was noted in the analysis of relative paw position. All rats placed their hind paws in a position approximately $16 \mathrm{~mm}$ after their previously placed front paw during walking (control: $17.7 \pm 2.7$ mm; sham: $14.2 \pm 2.9 \mathrm{~mm}$; bilateral 6-OHDA: $17.9 \pm 2.3 \mathrm{~mm}$ ).

\section{Stance phase}

The stance phase duration in the bilateral 6-OHDA group was significantly longer when compared to the control groups, for both forelimbs ( $p=0.001$ vs. control; $p=00.002$ vs. sham) and hind limbs ( $p=0.010$ vs. control; $p=0.014$ vs. sham; Fig. $4 A$ ). There was also a significant overall interaction between the groups and the test sessions for the forelimbs $(p=0.001)$ as well as the hind limbs ( $p=0.004)$. Post hoc analysis revealed that the difference was due to an effect of test within the bilateral 6-OHDA group (no test effect was seen in the control and sham groups). For the bilateral 6-OHDA rats, forelimb test session one and two differed from session three and four (p1 vs. $3=$ 0.005 ; p1 vs. $4<0.001$; p2 vs. $3=0.002$; p2 vs. $4<0.001$; see Fig. $5 \mathrm{~A}$ ). For the hind limbs, the difference was significant between test one and four $(p<0.001)$ and between test two and four $(p=0.009)$. As a consequence of the test effect in the 6-OHDA group, post hoc analysis also revealed that on the level of single test sessions, the overall group difference seen in forelimb stance between the two control groups (sham and normal controls) and bilateral 6-OHDA was present in test one ( $p<0.001 \mathrm{vs.} \mathrm{control} \mathrm{and} \mathrm{sham)}$ and in test two ( $p<0.001$ vs. control; $p=0.002 \mathrm{vs}$. sham). In test three the difference was only significant vs. the normal control group $(p=0.002)$, and in test four no differences was seen $(p>0.5)$. Thus, the longer stance phase for the bilateral group had disappeared by test four. The same scenario was seen for the hind limbs (test one: $p<$ 0.001 vs. both control and sham; test two: $p=0.012$ vs. control, $p=0.039$ vs. sham; test three: $p>0.1$ vs. both control and sham; test four: $p>0.5$ vs. both control and sham). 
A

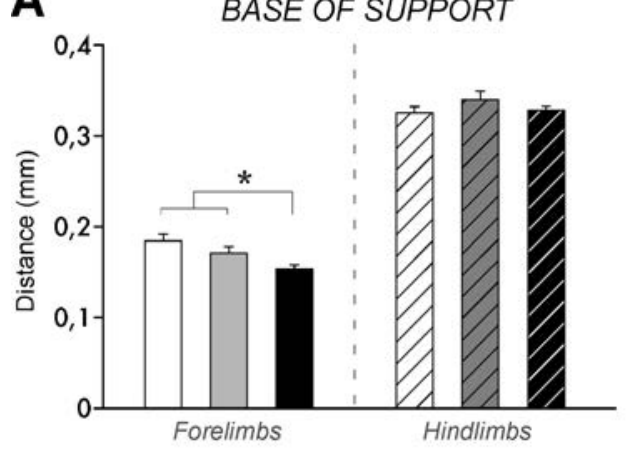

C

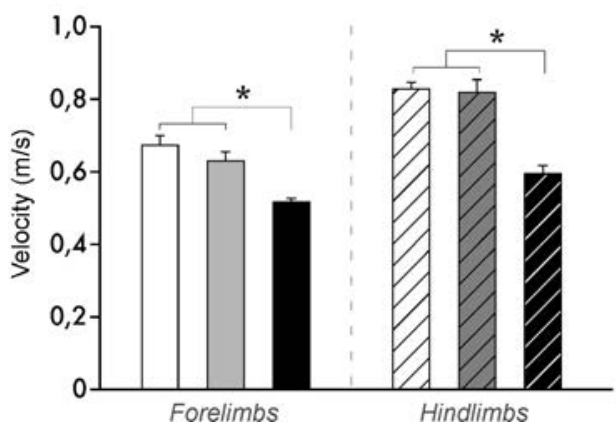

B

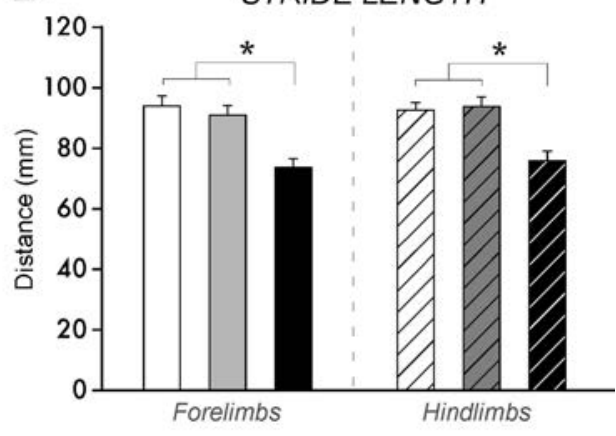

Fig. 3. Static paw parameters and swing speed were changed following bilateral 6-OHDA lesions. In bilaterally 6-OHDA lesioned rats the forelimb base of support was reduced (A). Furthermore both fore-and hind limb stride lengths (B) as well as swing speed (C) were reduced in the 6-OHDA rats. ${ }^{*} p<0.05$ compared to normal control and sham operated control rats.

\section{Swing phase}

Duration of the swing phase did not differ between the groups for either forelimbs or hind limbs, although a trend toward a longer swing phase in the bilateral 6-OHDA group was detected for the hind limbs ( $p=0.081$; Fig .4B). The duration of the swing phase for the forelimbs displayed a significant test effect, where the mean swing phase of test one $(0.153 \pm 0.009 \mathrm{~s})$ and test two $(0.150 \pm 0.009 \mathrm{~s})$ differed from the mean swing phase of test three $(0.189 \pm 0.009$ s; p1 vs. $3=0.028$; p2 vs. $3=0.027$. Test four $(0.179 \pm$ $0.009 \mathrm{~s})$ did not differ from the other trials. No interaction between group and test was found ( $p_{\text {front }}=0.317 ; p_{\text {hind }}=0.375$ ).

\section{Step cycle}

As a consequence of the prolonged stance duration within the bilateral 6-OHDA group, the step cycle (stance + swing) also differed between the groups. Bilateral 6-OHDA rats had a significantly longer step cycle than the two control groups in both forelimbs $(p<$ 
0.001 vs. control; $p=0.002$ vs. sham) and hind limbs ( $p=0.004$ vs. control; $p=0.005$ vs. sham; Fig. 4C). No test effect was present, but a group and test interaction was seen $\left(p_{\text {front }}=0.026 ; p_{\text {hind }}=0.013\right)$. The forelimb step cycle was longer for the bilateral rats in test one and two compared to test four (p1 vs. $4=0.020$; p2 vs. $4=0.021$; Fig. 5B). At the level of test session the bilateral rats had a significantly longer step cycle compared to control animals in test one ( $p<0.001$ vs. sham and control) and test two ( $p=0.003$ vs. sham and control), but the difference was gone by test three and four. Similarly, the hind limbs stance duration differed between test one and four in the bilateral 6-OHDA group (p1 vs. 4 = 0.006; Fig. 5B), manifested again as a prolonged step cycle duration for bilateral animals compared to controls in test one ( $p<0.001 \mathrm{vs}$. sham and control) and test two ( $p=0.007$ vs. control; $p=0.024$ vs. sham). The increased step cycle duration for the bilateral rats had disappeared by test three and four.

Duty cycle

Also the duty cycle (the percentage of the step cycle spent in stance phase) of the forelimbs was significantly higher in the bilateral 6-OHDA group compared to the normal control group ( $p=0.024)$ and to sham operated rats $(p=0.018$; Fig. 4D). There was an overall effect of test session within the forelimbs, where test two displayed a longer duty cycle $(69.4 \pm 0.75 \%)$ than test one $(66.9 \pm 0.75 \% ; p=0.023)$, test three $(64.6 \pm$ $0.75 \% ; p<0.001)$, and to test four $(65.4 \pm 0.75 \% ; p=0.001)$. Again, an overall group vs. test session interaction was found ( $p=0.001$; Fig. $5 C$ ), and it was due to differences present only within the bilateral 6-OHDA group. For bilateral rats test one and two were similar, but the forelimb duty cycle in the first and second test was significantly higher than in the fourth test ( $p 1$ vs. $4=0.002$; p2 vs. $4<0.001$ ). The second test displayed an increased duty cycle than the third ( $p 2$ vs. $3=0.002$ ) and there was a significant reduction of duty cycle in test four compared to test three $(p=0.028)$. When the interaction was investigated at the level of test sessions, the increase in duty cycle between bilateral 6-OHDA and control groups (Fig. 4D) were present in test one ( $p=0.028$ vs. control; $p=0.019$ vs. sham) and in test two ( $p=0.151$ vs. control; $p=0.010$ vs. sham), but difference between groups was gone in test three and four (Fig. 5C). The duty cycle did not differ between groups in total for the hind limbs (Fig. 4D), however there was an interaction between group and test session $(p=0.035)$. The interaction reflects a test effect within the bilateral 6-OHDA-group (Fig. 5C). The duty cycle of the hind limbs was reduced in test four compared to the first three test sessions ( 11 vs. $4=0.019$; p2 vs. $4=$ 0.001 ; p3 vs. $4=0.031$ ). On the level of single test sessions, there was no effect of group. 
A

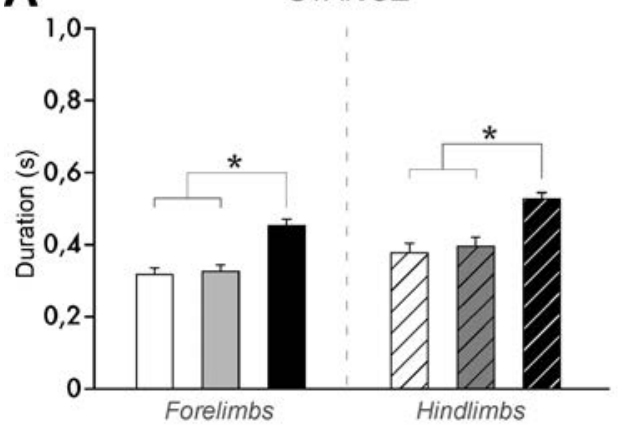

C

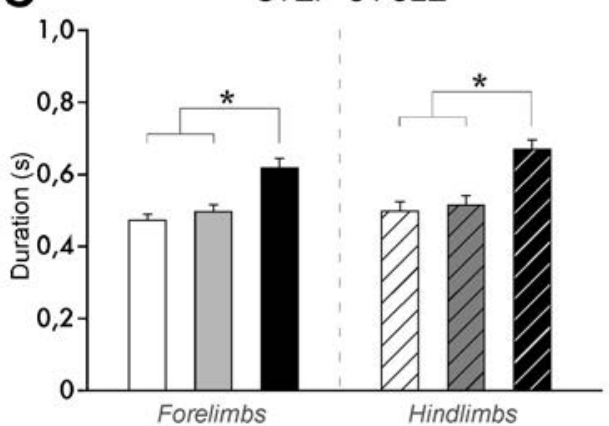

B

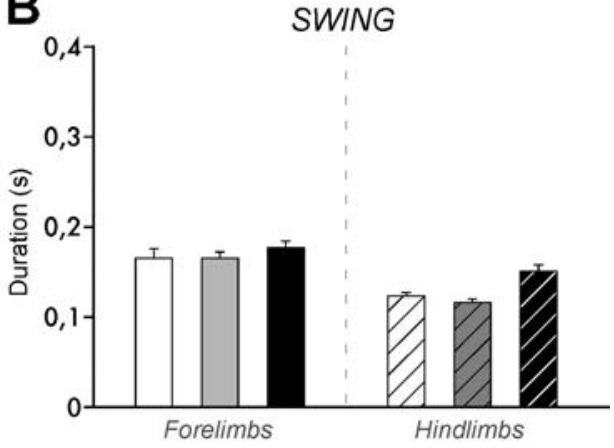

D DUTY CYCLE

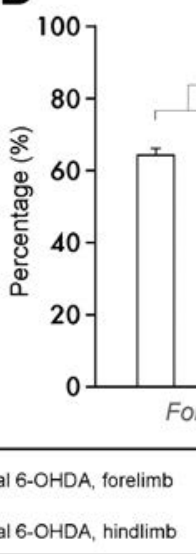

Fig. 4. Dynamic paw parameters were affected in the 6-OHDA lesioned rats. The stance phase was increased both for fore-and hind limbs in the 6-OHDA animals (A), whereas no effect was seen in the swing phase (B). The fore- and hind limb step cycle (C), and the forelimb duty cycle (D) were increased in the 6-OHDA rats. ${ }^{*} p<$ 0.05 compared to normal control and sham operated control rats.

Reversal of gait deficits with I-DOPA.

No difference between control and sham operated rats was observed on the two consecutive test days (2-way RM-ANOVA), thus data from test one and two was pooled for these groups. For technical reasons only six bilaterally 6-OHDA lesioned rats could be allocated to this study (only drug naïve rats were used in this experiment). An overview of the main findings is summarized in Table 2.

\section{Base of support}

There was a significant difference in the base of support between front and hind limbs $(p<0.001 ;$ Fig. 6A). As seen previously, there were no group differences between the hind limb BOS, but a trend for bilateral 6-OHDA rats to have a more narrow BOS ( $p=$ 0.056 vs. control; $p=0.061$ vs. sham). I-DOPA administration did not counteract the 
reduced forelimb $\mathrm{BOS}$, on the contrary the reduction became significant after the I-DOPA treatment ( $p=0.003$ vs. control; $p=0.002$ vs. sham).

A

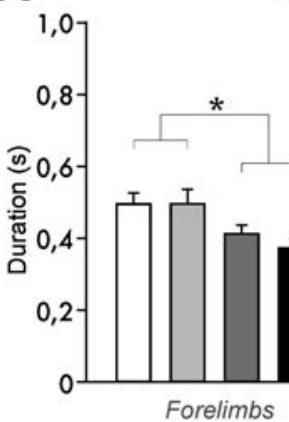

STANCE

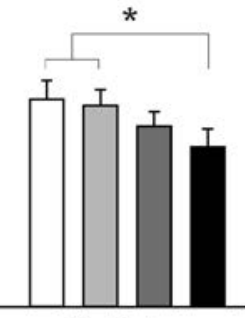

Hindlimbs

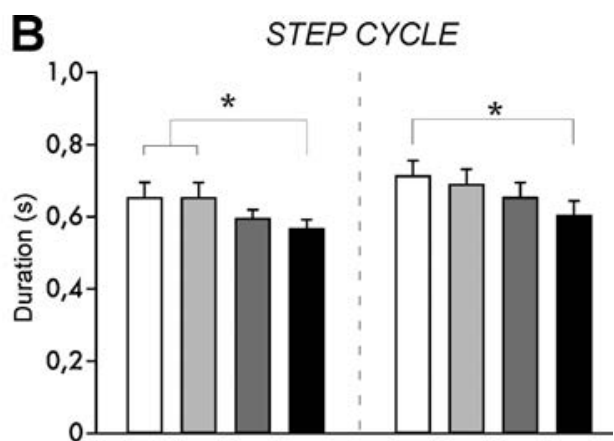

Forelimbs
Hindlimbs
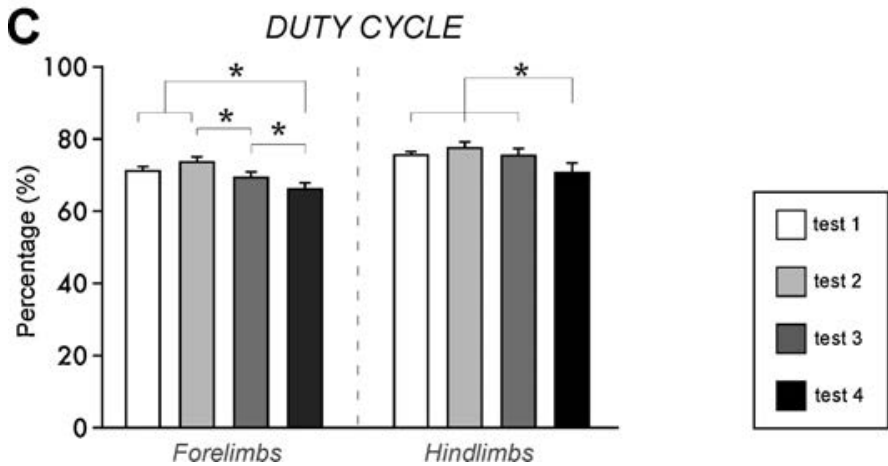

Fig. 5. Dynamic paw parameters changed in the 6-OHDA group during the time course of the study. The stance duration of both the fore- and the hind limbs had decreased by test four $(A)$, which was reflected in a concomitant decrease of the step cycle (B) and the duty cycle (C) in test four. ${ }^{*} p<0.05$ between compared groups.

Table 2. Summary of reversal of gait deficits with I-DOPA.

\begin{tabular}{|c|c|c|c|c|c|}
\hline & \multicolumn{3}{|c|}{ Vehicle 6-OHDA vs. sham } & \multicolumn{2}{|c|}{ I-DOPA in 6-OHDA vs. Sham } \\
\hline & Forelimb & Hindlimb & General & Forelimb & Hindlimb \\
\hline Base of support & - & - & & $\downarrow$ & - \\
\hline Stride length & $\downarrow$ & $\downarrow$ & & - & - \\
\hline Swing speed & $\downarrow$ & $\downarrow$ & & - & - \\
\hline Regularity index & & & - & & \\
\hline Stance phase & $\uparrow$ & $\uparrow$ & & - & - \\
\hline Swing phase & - & - & & - & - \\
\hline Step cycle & & & $\uparrow$ & & \\
\hline
\end{tabular}

$\downarrow$ : Significant decrease, $\uparrow$ : significant increase, - - no significant change 


\section{Stride length}

The stride length was equal between forelimbs and hind limbswithin each test group (Fig. 6B). As in the previous experiment, stride length was reduced in rats with bilateral $6-O H D A$ lesions ( $p<0.001$ vs. control and sham; compare black bars in Figs. 3B and 6B). Interestingly, there was a significant improvement in bilateral rats after I-DOPA administration ( $p=0.032$ vs. untreated 6-OHDA group; Fig. 6B). No statistical difference between control groups and bilateral + I-DOPA group was found (Fig. 6B; compare white and grey bars to checked bars, $p=0.52$ and $p=0.77$, respectively).

\section{Swing speed}

Analysis of swing speed showed a highly significant difference between the hind and the forelimbs ( $p<0.001$; Fig. $6 C$ ). Again, a reduction of both hind and forelimb swing speed in the bilaterally 6-OHDA lesioned animals was present ( $p=0.002$ vs. control; $p=0.003$ vs. sham; compare to Fig. 3C). After I-DOPA treatment the significance was gone (Fig. 6C; compare white and grey bars to checked bars, $p=0.688$ and $p=0.577$, respectively).

\subsubsection{Regularity index}

The regularity index analysis showed a difference between groups (control: $97.63 \pm$ 1.06\%; sham: $97.96 \pm 0.86 \%$; bilateral: $93.79 \pm 1.37 \%$; bilateral + I-DOPA: $97.73 \pm 1.09 \%$; $p=0.044)$, but post hoc Newman-Keuls multiple comparisons did not reach statistical significance.

\section{Stance phase}

The duration of the stance phase did not differ between the forelimb and the hind limb $(p=0.07)$, but the bilateral 6-OHDA rats had an increased stance duration of both forelimbs and hind limbs ( $p=0.005$ vs. control and sham; Fig. 6D). I-DOPA reduced the stance duration so that the difference to control groups was abolished ( $p=0.29$ vs. control and 0.41 vs. sham).

\section{Swing phase}

There was no group difference in the duration of swing phase, either in hind or forelimbs, however the mean swing duration of forelimbs differed from the swing phase of the hind limbs (0.164 \pm 0.0052 s vs. $0.128 \pm 0.0052 \mathrm{~s} ; \mathrm{p}<0.001)$.

\section{Step cycle}

The stance result was reflected in duration of the whole step cycle, so that bilateral 6OHDA had a prolonged step cycle compared to control groups ( $p=0.006$ vs. control and sham; Fig. 6E), and I-DOPA reduced the step cycle so that the statistical difference disappeared ( $p=0.30$ vs. control and $p=0.44$ vs. sham). 
A

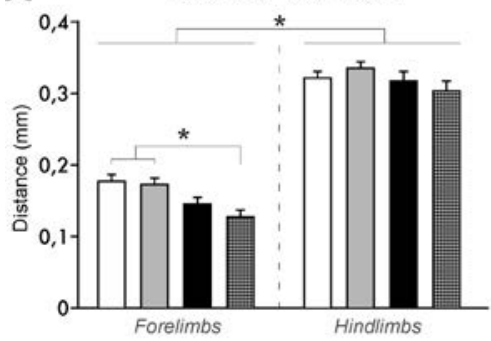

C

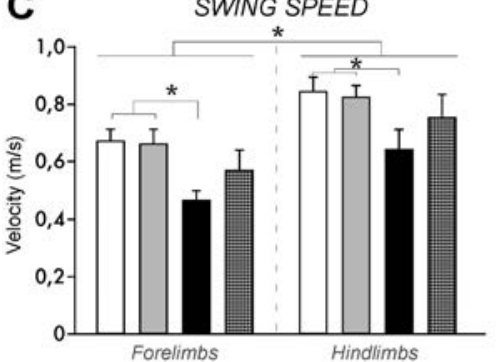

E

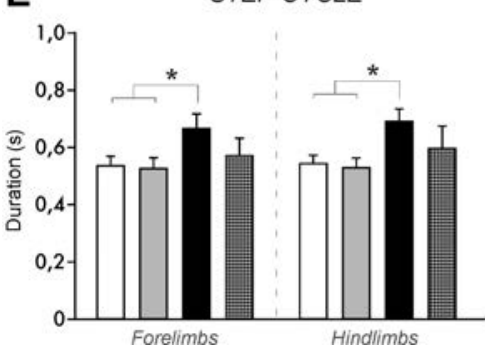

B

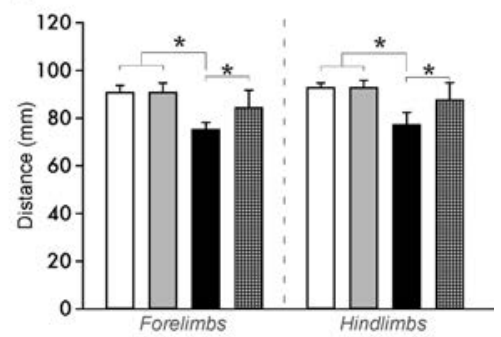

D

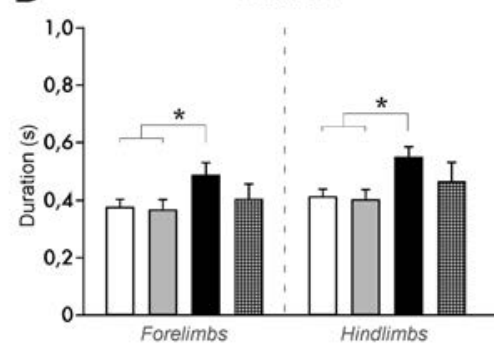

Fig. 6. Effect of I-DOPA treatment on gait deficits. A single dose of I-DOPA (10 mg/kg) worsened the forelimb base of support (A), but could restore both fore-and hind limb stride length in 6-OHDA lesioned rats (B). I-DOPA could counteract the dopamine mediated impairment seen in both fore- and hind limb performance of swing speed (C), stance duration (D), and Step cycle duration $(E)$ in the $6-O H D A$ rats. ${ }^{*} p<0.05$ between compared groups.

\section{Discussion}

In this study we demonstrated that the CatWalk is a constant and reliable tool to investigate multiple gait parameters quantitatively. Furthermore, we investigated the gait deficits in a bilateral 6-OHDA animal model mimicking advanced stages of PD. Gait was generally disturbed in DA depleted animals which was reflected by changes in various gait parameters. L-DOPA therapy partially reversed some, but not all, disturbed gait parameters. Below, we will discuss the reproducibility of gait patterns with the CatWalk 
system, gait changes in the experimental PD model, and the potential of I-DOPA to reverse gait disturbances.

\section{Constancy of gait patterns with the CatWalk system}

The CatWalk analyses revealed a high degree of constancy and reproducibility in how a crossing of the runway was executed by (neurologically) intact rats. Through all four test sessions, the parameters investigated displayed similar numerical output for both control and sham operated rats, irrespective of the fact that body weight varied to some degree between the test groups and test sessions. Inter-test variation of hind limb performance was never present within these groups. Forelimb performance of the control groups was to some extent less uniform, since the swing phase (and thereby the duty cycle) showed some variability between tests. In this aspect it is noteworthy that the variation was not large enough to affect the average duration of the step cycle between tests. To conclude, all parameters but forelimb swing are stable over time in neurologically intact rats, and are thus suitable to investigate in multiple testing paradigms.

The second experiment (I-DOPA) allowed for comparison between front and hind limbs, and there was indeed a significantdifference between hind and forelimb dynamics in both base of support, swing phase, and swing speed (see Fig. 5). This is in agreement with what is visualized, but not statistically analyzed in other studies (Koopmans, et al., 2007). Based on these findings, the CatWalk proofed to be an objective tool to investigate the effect of pharmacologic and invasive treatments on gait.

\section{Bilateral 6-OHDA mfb lesions as a model for experimental PD}

Aware of the limitations of the bilateral 6-OHDA model, which mimics a DA disorder rather than the multisystem disorder of PD, we chose to use the best available rat model. We investigated bilateral 6-OHDA lesions, as toxin injections into the $\mathrm{mfb}$ to destroy DA neurons that terminate in the corpus striatum, and to some extent also neurons of the mesolimbic and mesocortical pathways. The rationale was to avoid the smaller, more compartmental lesions created by striatal infusions of 6-OHDA (where the injection sites are typically selected so that DA neurons innervating the lateral parts of striatum are destroyed, whereas neurons that innervate the more medial areas are spared). The aim was to mimic a stage of PD where there are symptoms of motor deficits, but the subject is still able to perform tasks like walking. The obtained degree of DA denervation is in line with previous studies, where bilateral mfb injections of $6 \mathrm{~g}$ 6-OHDA resulted in a 75\% mean DA depletion (Srinivasan and Schmidt, 2004) and more than $50 \%$ loss of SNc DA neurons, which is equal to the human situation (Hornykiewicz, 1975, Marsden, 1990). For each individual rat, the DA depletion was asymmetric between the striata, which reflects the clinical situation well (Hoehn and Yahr, 1967). A correlation was seen between SNc cell count and the amount of DA depletion in the striatum. To 
our knowledge the correlation we found between SNc cell loss and DA depletion in the striatum has never been reported before in the bilateral 6-OHDA model.

The striatal NA levels are reduced by about 70\%, a similar reduction as seen for DA. The LC-noradrenergic system has a facilitatory influence on the function of the nigrostriatal DA system (Yavich, et al., 2003), however NA deficiency is mainly thought to underlie depression-like symptoms in PD (Gerlach, et al., 1994).

\section{Gait in $P D$}

Clinically, PD induces gait disturbances such as reduced overall velocity, decreased arm swing, reduced stride length, and increased duration of the stance phase (Blin, et al., 1991, Knutsson, 1972, Stern, et al., 1983). To interpret data from experimental 6-OHDA lesioned quadrupedal rodents and extrapolate this to the (bipedal) PD patient is a hazardous and difficult task. The dynamic parameter stance illustrates the pitfalls well, where PD patients have an increased stance duration of the part spend in double limb support (i.e. floor contact with both feet) (Krystkowiak, et al., 2001). The stance duration of a rat paw does not take the position of the other paws into consideration: it comprises single, double, triple and quadruple limb support. Thus, that the stance phase is initially prolonged in PD rats does not have any straightforward similarity to what is observed in patients. In this respect, swing speed and stride length may be more readily comparable as the length of a step and the speed it is conducted with are irrespective of the simultaneous behaviours of the other paws. Still, the translation of the gait parameters to the clinical situation has to be done with caution.

Rats subjected to bilateral 6-OHDA lesions had difficulties to perform an uninterrupted crossing of the runway. To our opinion these interruptions could be seen as "freezing" behaviour as present in PD patients (Kemoun and Defebvre, 2001). Due to this "freezing" behaviour, the inclusion criteria had to be modified. This meant that it was not possible to obtain and compare the time to cross the walkway (speed) between groups. Despite the loss of this parameter, several other aspects of gait could still be investigated. We found alterations in most parameters examined, which proved that a reduction of bilateral striatal DA (and NA) levels by $70 \%$ had a substantial effect on gait. The denervation following bilateral mfb infusion affected performance of both the forelimbs and the hind limbs (with exception of BOS), an interesting phenomenon as previous literature reports only changes in the forelimbs after unilateral mfb lesion (Vandeputte, et al., 2010) and mainly in the hind limbs after bilateral striatal 6-OHDA infusion (Vlamings, et al., 2007).

A closer look at the static gait parameter showed that the stride length was reduced by approximately $20 \%$ for both forelimbs and hind limbs. This is similar to the clinical situation, in which PD patients make smaller steps (Kemoun and Defebvre, 2001). The relative paw position was not changed in the lesioned rats, which means 
that the spatial dynamics between the ipsilateral hind limb and forelimb paw placements were not affected by the reduced stride length. However, the reduced forelimb base of support displays an effect of the lesion in the interlimb placement of the fore paws. Duration of the dynamic parameters stance and step cycle were prolonged in both fore- and hind limbs of the lesioned rats, whereas no statistical differences were seen in the swing duration of the forelimbs and only a trend to a prolonged swing phase of the hind limbs. The swing phase trend affected the hind limb duty cycle (the percentage of the step cycle spent in stance phase), as it counterbalanced the prolongation of stance, such that no difference between the groups was present. On the contrary, the prolonged stance of the forelimb impacted the duty cycle of the forelimbs, thus it was increased for the bilateral 6-OHDA rats. An important finding during the data analysis was that the prolonged stance duration of the lesioned rats was not stable over time. The duration gradually decreased with each test session. Therefore, the initial significant increase of stance duration in relation to control rats had disappeared by the last session. The normalization toward control rats was also reflected in a normalization of the step and duty cycle by test three and four. The knowledge that stance was not stable over time, warrants caution if it will be used in long-time studies.

Our finding that the swing speed was reduced in bilateral 6- OHDA rats was expected, as the swing phase duration was similar between groups (there even a trend to a prolonged swing phase in the hind limbs), but the distance the paw moved was decreased, hence the speed had to be reduced as well. Furthermore, there was strong indirect evidence that the average locomotor velocity in the PD rats was reduced as well; the duration of a step cycle was longer, the length of a step shorter, and the swing speed of the paw slower. Thus, for each step a bilateral 6-OHDA rat took, its body was moved a shorter distance during a longer time span in comparison to a normal rat. The left-right coordination, reflected in the regularity index, was slightly decreased in bilateral lesioned rats, but not to the extent seen after for example unilateral spinal cord injury [(Hamers, et al., 2001, Klapka, et al., 2005).

\section{The effect of I-DOPA gait deficits}

I-DOPA treatment restored the stride length to almost the same distance as seen in intact rats. However, it only partially reversed the reduced swing speed and the prolonged step cycle seen in the DA lesioned rats. This could be due to fact that the dose was below optimal, but fits with the effects of I-DOPA measured in PD patients (Klawans, 1986). The administered dose was chosen in the lower range of the therapeutic span to avoid the appearance of dyskinetic side effects upon de novo administration (Cenci, et al., 1998). It is noteworthy that the PD rats again displayed a prolonged stance phase in the I-DOPA experiment, which took place four weeks after the last test session of the former experiment. This indicates that the normalization of stance that gradually 
took place in the repeated experiment required recurrent weekly exposure to the CatWalk, rather than representing a late stage compensatory change of motor function as a result of the DA denervation. The restored stride length after I-DOPA treatment is in line with clinical findings, where stride length and kinematic parameters respond to IDOPA (Blin, et al., 1991, Lubik, et al., 2006). The base of support was not affected by IDOPA, which indicates that this could be a DA-resistant gait parameter. Interestingly, a previous study reported an increased forelimb BOS in parkinsonian rats after bilateral high frequency stimulation of the subthalamic nucleus (Vlamings, et al., 2007). Our findings again underline the limited role of I-DOPA therapy for axial symptoms in PD. Therefore the research focus should extend beyond DA, to investigate the pathophysiological role of other neurochemical changes. Possible target receptor distribution in the motor circuit for novel drug therapies should be mapped. And second, the focus should move from the basal ganglia nuclei to the role of brain stem and cerebellar areas, and maybe even the spinal cord.

\section{Conclusion}

This experiment has shown that the CatWalk method is a very reproducible tool to assess voluntary walking in neurologically intact as well as Parkinsonian rats. Rats with a $70 \%$ reduction in striatal DA showed gait disturbances, but not all deficits were stable over time and the translation of quadrupal to bipedal gait remains hazardous. L-DOPA therapy had good effects on gait, but not all parameters improved. In view of the fact that gait disturbances are a major cause of morbidity and even mortality in PD, further preclinical investigations to find underlying mechanisms for gait problems in PD, to provide novel therapeutic approaches, is needed.

\section{Acknowledgements}

The behavioural part of the study was fully sponsored by $\mathrm{H}$-Lundbeck $\mathrm{A} / \mathrm{S}$ and performed in Valby. The authors would like to thank Maria Ronild and Marlene Quvang Jorgensen for their excellent technical assistance. 


\section{References}

1. Amende, I., Kale, A., McCue, S., Glazier, S., Morgan, J. P., and Hampton, T. G., 2005. Gait dynamics in mouse models of Parkinson's disease and Huntington's disease. Journal of neuroengineering and rehabilitation 2, 20.

2. Blin, O., Ferrandez, A. M., Pailhous, J., and Serratrice, G., 1991. Dopa-sensitive and dopa-resistant gait parameters in Parkinson's disease. Journal of the neurological sciences 103, 51-54.

3. Blin, O., Ferrandez, A. M., and Serratrice, G., 1990. Quantitative analysis of gait in Parkinson patients: increased variability of stride length. Journal of the neurological sciences 98, 91-97.

4. Browner, N., and Giladi, N., 2010. What can we learn from freezing of gait in Parkinson's disease? Current neurology and neuroscience reports 10, 345-351.

5. Carter, R. J., Lione, L. A., Humby, T., Mangiarini, L., Mahal, A., Bates, G. P., Dunnett, S. B., and Morton, A. J., 1999. Characterization of progressive motor deficits in mice transgenic for the human Huntington's disease mutation. The Journal of neuroscience : the official journal of the Society for Neuroscience 19, 3248-3257.

6. Cenci, M. A., Lee, C. S., and Bjorklund, A., 1998. L-DOPA-induced dyskinesia in the rat is associated with striatal overexpression of prodynorphin- and glutamic acid decarboxylase mRNA. Eur J Neurosci 10, 2694-2706.

7. Cenci, M. A., and Ohlin, K. E., 2009. Rodent models of treatment-induced motor complications in Parkinson's disease. Parkinsonism Relat Disord 15 Suppl 4, S13-17.

8. Cenci, M. A., Whishaw, I. Q., and Schallert, T., 2002. Animal models of neurological deficits: how relevant is the rat? Nature reviews. Neuroscience 3, 574-579.

9. Chang, J. Y., Shi, L. H., Luo, F., and Woodward, D. J., 2003. High frequency stimulation of the subthalamic nucleus improves treadmill locomotion in unilateral 6-hydroxydopamine lesioned rats. Brain Res 983, 174-184.

10. Chuang, C. S., Su, H. L., Cheng, F. C., Hsu, S. H., Chuang, C. F., and Liu, C. S., 2010. Quantitative evaluation of motor function before and after engraftment of dopaminergic neurons in a rat model of Parkinson's disease. Journal of biomedical science 17, 9.

11. Dauer, W., and Przedborski, S., 2003. Parkinson's disease: mechanisms and models. Neuron 39, 889-909.

12. de Medinaceli, L., Freed, W. J., and Wyatt, R. J., 1982. An index of the functional condition of rat sciatic nerve based on measurements made from walking tracks. Exp Neurol 77, 634-643.

13. Deumens, R., Jaken, R. J., Marcus, M. A., and Joosten, E. A., 2007. The CatWalk gait analysis in assessment of both dynamic and static gait changes after adult rat sciatic nerve resection. J Neurosci Methods 164, 120-130.

14. Devos, D., Defebvre, L., and Bordet, R., 2010. Dopaminergic and non-dopaminergic pharmacological hypotheses for gait disorders in Parkinson's disease. Fundamental \& clinical pharmacology 24, 407-421.

15. Fleming, S. M., Salcedo, J., Fernagut, P. O., Rockenstein, E., Masliah, E., Levine, M. S., and Chesselet, M. F., 2004. Early and progressive sensorimotor anomalies in mice overexpressing wild-type human alphasynuclein. The Journal of neuroscience : the official journal of the Society for Neuroscience 24, 94349440.

16. Gabriel, A. F., Marcus, M. A., Honig, W. M., Walenkamp, G. H., and Joosten, E. A., 2007. The CatWalk method: a detailed analysis of behavioral changes after acute inflammatory pain in the rat. J Neurosci Methods 163, 9-16.

17. Gabriel, A. F., Marcus, M. A., Walenkamp, G. H., and Joosten, E. A., 2009. The CatWalk method: assessment of mechanical allodynia in experimental chronic pain. Behav Brain Res 198, 477-480.

18. Gelb, D. J., Oliver, E., and Gilman, S., 1999. Diagnostic criteria for Parkinson disease. Arch Neurol 56, 3339.

19. Gensel, J. C., Tovar, C. A., Hamers, F. P., Deibert, R. J., Beattie, M. S., and Bresnahan, J. C., 2006. Behavioral and histological characterization of unilateral cervical spinal cord contusion injury in rats. J Neurotrauma 23, 36-54. 
20. Gerlach, M., Jellinger, K., and Riederer, P., 1994. The possible role of noradrenergic deficits in selected signs of Parkinson's Disease. CRC Press, 59-72.

21. Hamers, F. P., Koopmans, G. C., and Joosten, E. A., 2006. CatWalk-assisted gait analysis in the assessment of spinal cord injury. J Neurotrauma 23, 537-548.

22. Hamers, F. P., Lankhorst, A. J., van Laar, T. J., Veldhuis, W. B., and Gispen, W. H., 2001. Automated quantitative gait analysis during overground locomotion in the rat: its application to spinal cord contusion and transection injuries. J Neurotrauma 18, 187-201.

23. Hoehn, M. M., and Yahr, M. D., 1967. Parkinsonism: onset, progression and mortality. Neurology 17, 427-442.

24. Hornykiewicz, O., 1975. Brain monoamines and Parkinsonism. Psychopharmacology bulletin 11, 34-35.

25. Karachi, C., Grabli, D., Bernard, F. A., Tande, D., Wattiez, N., Belaid, H., Bardinet, E., Prigent, A., Nothacker, H. P., Hunot, S., Hartmann, A., Lehericy, S., Hirsch, E. C., and Francois, C., 2010. Cholinergic mesencephalic neurons are involved in gait and postural disorders in Parkinson disease. The Journal of clinical investigation 120, 2745-2754.

26. Kemoun, G., and Defebvre, L., 2001. [Gait disorders in Parkinson disease. Clinical description, analysis of posture, initiation of stabilized gait]. Presse medicale 30, 452-459.

27. Kemoun, G., and Defebvre, L., 2001. [Gait disorders in Parkinson disease. Gait freezing and falls: therapeutic management]. Presse medicale 30, 460-468.

28. Klapka, N., Hermanns, S., Straten, G., Masanneck, C., Duis, S., Hamers, F. P., Muller, D., Zuschratter, W., and Muller, H. W., 2005. Suppression of fibrous scarring in spinal cord injury of rat promotes longdistance regeneration of corticospinal tract axons, rescue of primary motoneurons in somatosensory cortex and significant functional recovery. Eur J Neurosci 22, 3047-3058.

29. Klawans, H. L., 1986. Individual manifestations of Parkinson's disease after ten or more years of levodopa. Mov Disord 1, 187-192.

30. Klein, A., Wessolleck, J., Papazoglou, A., Metz, G. A., and Nikkhah, G., 2009. Walking pattern analysis after unilateral 6-OHDA lesion and transplantation of foetal dopaminergic progenitor cells in rats. Behav Brain Res 199, 317-325.

31. Knutsson, E., 1972. An analysis of Parkinsonian gait. Brain : a journal of neurology 95, 475-486.

32. Koopmans, G. C., Deumens, R., Brook, G., Gerver, J., Honig, W. M., Hamers, F. P., and Joosten, E. A., 2007. Strain and locomotor speed affect over-ground locomotion in intact rats. Physiology \& behavior 92, 993-1001.

33. Koopmans, G. C., Deumens, R., Honig, W. M., Hamers, F. P., Steinbusch, H. W., and Joosten, E. A., 2005. The assessment of locomotor function in spinal cord injured rats: the importance of objective analysis of coordination. J Neurotrauma 22, 214-225.

34. Krystkowiak, P., Blatt, J. L., Bourriez, J. L., Duhamel, A., Perina, M., Kemoun, G., Blond, S., Guieu, J. D., Destee, A., and Defebvre, L., 2001. Chronic bilateral pallidal stimulation and levodopa do not improve gait in the same way in Parkinson's disease: a study using a video motion analysis system. J Neurol 248, 944-949.

35. Lubik, S., Fogel, W., Tronnier, V., Krause, M., Konig, J., and Jost, W. H., 2006. Gait analysis in patients with advanced Parkinson disease: different or additive effects on gait induced by levodopa and chronic STN stimulation. J Neural Transm Park Dis Dement Sect 113, 163-173.

36. Marsden, C. D., 1990. Parkinson's disease. Lancet 335, 948-952.

37. Mazarakis, N. K., Cybulska-Klosowicz, A., Grote, H., Pang, T., Van Dellen, A., Kossut, M., Blakemore, C., and Hannan, A. J., 2005. Deficits in experience-dependent cortical plasticity and sensory-discrimination learning in presymptomatic Huntington's disease mice. The Journal of neuroscience : the official journal of the Society for Neuroscience 25, 3059-3066.

38. Meredith, G. E., Totterdell, S., Potashkin, J. A., and Surmeier, D. J., 2008. Modeling PD pathogenesis in mice: advantages of a chronic MPTP protocol. Parkinsonism Relat Disord 14 Suppl 2, S112-115.

39. Metz, G. A., Tse, A., Ballermann, M., Smith, L. K., and Fouad, K., 2005. The unilateral 6-OHDA rat model of Parkinson's disease revisited: an electromyographic and behavioural analysis. Eur J Neurosci 22, 735744. 
40. Metz, G. A., and Whishaw, I. Q., 2002. Cortical and subcortical lesions impair skilled walking in the ladder rung walking test: a new task to evaluate fore- and hindlimb stepping, placing, and co-ordination. J Neurosci Methods 115, 169-179.

41. Metz, G. A., and Whishaw, I. Q., 2009. The ladder rung walking task: a scoring system and its practical application. Journal of visualized experiments : JoVE.

42. Morris, M. E., lansek, R., Matyas, T. A., and Summers, J. J., 1994. The pathogenesis of gait hypokinesia in Parkinson's disease. Brain : a journal of neurology 117 ( Pt 5), 1169-1181.

43. Paxinos, G., and Watson, C., 1998. The Rat Brain in Stereotaxic Coordinates. Academic Press, New York.

44. Pullman, S. L., Watts, R. L., Juncos, J. L., Chase, T. N., and Sanes, J. N., 1988. Dopaminergic effects on simple and choice reaction time performance in Parkinson's disease. Neurology 38, 249-254.

45. Schmitz, C., and Hof, P. R., 2005. Design-based stereology in neuroscience. Neuroscience 130, 813-831.

46. Shi, L. H., Luo, F., Woodward, D. J., and Chang, J. Y., 2006. Basal ganglia neural responses during behaviorally effective deep brain stimulation of the subthalamic nucleus in rats performing a treadmill locomotion test. Synapse 59, 445-457.

47. Shi, L. H., Woodward, D. J., Luo, F., Anstrom, K., Schallert, T., and Chang, J. Y., 2004. High-frequency stimulation of the subthalamic nucleus reverses limb-use asymmetry in rats with unilateral 6hydroxydopamine lesions. Brain Res 1013, 98-106.

48. Srinivasan, J., and Schmidt, W. J., 2004. Behavioral and neurochemical effects of noradrenergic depletions with $\mathrm{N}$-(2-chloroethyl)-N-ethyl-2-bromobenzylamine in 6-hydroxydopamine-induced rat model of Parkinson's disease. Behav Brain Res 151, 191-199.

49. Stern, G. M., Franklyn, S. E., Imms, F. J., and Prestidge, S. P., 1983. Quantitative assessments of gait and mobility in Parkinson's disease. Journal of neural transmission. Supplementum 19, 201-214.

50. Tan, S. K., Hartung, H., Sharp, T., and Temel, Y., 2011. Serotonin-dependent depression in Parkinson's disease: A role for the subthalamic nucleus? Neuropharmacology.

51. Taylor, T. N., Greene, J. G., and Miller, G. W., 2010. Behavioral phenotyping of mouse models of Parkinson's disease. Behav Brain Res 211, 1-10.

52. Temel, Y., Visser-Vandewalle, V., Aendekerk, B., Rutten, B., Tan, S., Scholtissen, B., Schmitz, C., Blokland, A., and Steinbusch, H. W., 2005. Acute and separate modulation of motor and cognitive performance in parkinsonian rats by bilateral stimulation of the subthalamic nucleus. Exp Neurol 193, 43-52.

53. Tillerson, J. L., Cohen, A. D., Philhower, J., Miller, G. W., Zigmond, M. J., and Schallert, T., 2001. Forced limb-use effects on the behavioral and neurochemical effects of 6-hydroxydopamine. The Journal of neuroscience : the official journal of the Society for Neuroscience 21, 4427-4435.

54. Vandeputte, C., Taymans, J. M., Casteels, C., Coun, F., Ni, Y., Van Laere, K., and Baekelandt, V., 2010. Automated quantitative gait analysis in animal models of movement disorders. BMC neuroscience 11, 92.

55. Vlamings, R., Visser-Vandewalle, V., Koopmans, G., Joosten, E. A., Kozan, R., Kaplan, S., Steinbusch, H. W., and Temel, Y., 2007. High frequency stimulation of the subthalamic nucleus improves speed of locomotion but impairs forelimb movement in Parkinsonian rats. Neuroscience 148, 815-823.

56. Wenning, G. K., Ebersbach, G., Verny, M., Chaudhuri, K. R., Jellinger, K., McKee, A., Poewe, W., and Litvan, I., 1999. Progression of falls in postmortem-confirmed parkinsonian disorders. Movement disorders : official journal of the Movement Disorder Society 14, 947-950.

57. Williams, A., Gill, S., Varma, T., Jenkinson, C., Quinn, N., Mitchell, R., Scott, R., Ives, N., Rick, C., Daniels, J., Patel, S., and Wheatley, K., 2010. Deep brain stimulation plus best medical therapy versus best medical therapy alone for advanced Parkinson's disease (PD SURG trial): a randomised, open-label trial. Lancet Neurol 9, 581-591.

58. Yavich, L., Sirvio, J., Haapalinna, A., Ylinen, A., and Mannisto, P. T., 2003. Atipamezole, an alpha2adrenoceptor antagonist, augments the effects of L-DOPA on evoked dopamine release in rat striatum. European journal of pharmacology 462, 83-89.

59. Yuan, H., Zhang, Z. W., Liang, L. W., Shen, Q., Wang, X. D., Ren, S. M., Ma, H. J., Jiao, S. J., and Liu, P., 2010. Treatment strategies for Parkinson's disease. Neuroscience bulletin 26, 66-76. 
60. Zarow, C., Lyness, S. A., Mortimer, J. A., and Chui, H. C., 2003. Neuronal loss is greater in the locus coeruleus than nucleus basalis and substantia nigra in Alzheimer and Parkinson diseases. Arch Neurol 60, 337-341. 



\section{CHAPTER 9}

\section{Mild dopaminergic lesions are accompanied by robust changes in subthalamic nucleus activity.}

Janssen MLF, Zwartjes DGM, Tan SKH, Vlamings R, Jahanshahi A, Heida T, Hoogland G, Steinbusch H, Visser-Vandewalle $V$, Temel $Y$

Neuroscience Letters 2012 Febr;508(2):101-105. 


\section{Abstract}

The subthalamic nucleus (STN) is a major player in the input and output of the basal ganglia motor circuitry. The neuronal regular firing pattern of the STN changes into a pathological bursting mode in both advanced Parkinson's Disease (PD) and in PD animals models with severe dopamine depletion. One of the current hypothesis, based on clinical and experimental evidence, is that this typical burst activity is responsible for some of the principal motor symptoms. In the current study we tested whether mild DA depletion, mimicking early stages of PD, induced deficits in motor behaviour and changes in STN neuronal activity. The present study demonstrated that rats with a mild lesion (20-40 \% loss of DA neurons) and a slowed motor response, but without gross motor abnormalities already have an increased number of bursty STN neurons under urethane anaesthesia. These findings indicate that the early increase in STN burst activity is a compensatory mechanism to maintain the dopamine homeostasis in the basal ganglia. 


\section{Introduction}

The subthalamic nucleus (STN), is a major player in the input and output of the basal ganglia motor circuitry (Albin, et al., 1989, Smith, et al., 1998). Firstly, the STN forms together with the striatum the basal ganglia entry sites for cortical input (Afsharpour, 1985, Monakow, et al., 1978). Secondly, the STN strongly modulates the basal ganglia output nuclei by its glutamatergic efferents and is also an output nucleus itself by its direct cortical projections (Carpenter, et al., 1981, Ricardo, 1980).

Animal studies indicate that STN neurons normally fire in a regular manner (Nakanishi, et al., 1987, Overton and Greenfield, 1995, Wichmann, et al., 1994). In both PD patients and severe animal models of PD, the STN's neuronal regular firing pattern changes into a pathological bursting mode (Benazzouz, et al., 2002, Bergman, et al., 1994). One of the current hypothesis is that this typical burst activity is responsible for some of the principal motor symptoms (Benazzouz, et al., 1992, Bergman, et al., 1994, Hutchison, et al., 1998, Rodriguez, et al., 1998). Both, clinical and experimental evidence support this hypothesis. For instance, intra-operatively collected electrophysiological recordings from the STN of PD patients show a typical burst pattern and modulation of this pattern by deep brain stimulation (DBS) alleviates motor disability (Benabid, et al., 1994, Krack, et al., 2003, Visser-Vandewalle, et al., 2005). Also animal models of PD are characterized by a pathological firing pattern of STN neurons (Bergman, et al., 1994, Hollerman and Grace, 1992, Ni, et al., 2001).

Although altered STN activity is observed in PD patients in the advanced stages of the disease as well as in animal models with severe DA lesions, it is still elusive at which stage of the disease changes in STN neuronal activity occur. In the current study, we addressed this by evaluating electrophysiological characteristics of STN neurons and the motor behaviour in an animal model mimicked early stages of PD by applying different levels of dopamine (DA) depletion by bilateral striatal 6OHDA injections. Electrophysiological changes were assessed using in vivo, single-cell extracellular recordings in the STN. Motor behaviour was evaluated by reaction time task (Temel, et al., 2005) and locomotor activity in by the open field task (Hameleers, et al., 2007), and the CatWalk automated gait analysis task (Vlamings, et al., 2007).

\section{Material and Methods}

\section{Subjects}

Male Lewis rats ( $N=30,10$ weeks old) bred and housed at the Central Animal Facility of Maastricht University (Maastricht, The Netherlands), were 15 weeks old and weighed $\sim 315 \mathrm{~g}$ at the time of surgery. All experimental procedures were approved by the Animal Experiments and Ethics Committee of Maastricht University. 


\section{Surgical procedure}

Two rats died peri-operatively, and the others were divided in the following four experimental groups: A. sham ( $n=7)$; B. $2.5 \mu \mathrm{g} / \mu \mathrm{l}$ 6-OHDA ( $=7$ ); C. $5 \mu \mathrm{g} / \mu \mathrm{l}$ 6-OHDA ( $=7$ ); D. $7.5 \mu \mathrm{g} \mu \mathrm{l}$ 6-OHDA ( $\mathrm{n}=7$ ). These three different concentrations were chosen to assess changes in motor behaviour and electrophysiological activity in mild to moderate DA depleted animals mimicking different stages of PD. Details of the surgical procedure have been described earlier (Tan, et al., 2010). In brief, one hour before surgery rats were injected with Temgesic $(0.1 \mathrm{mg} / \mathrm{kg}$, s.c.) and with desimipramine (20 mg/kg, i.p.). Throughout surgery, rats were anesthetized by $2 \%$ isoflurane inhalation. Rats were bilaterally injected in the striatum with $2 \mu \mathrm{l}$ vehicle $0.2 \%$ ascorbic acid dissolved in $0.9 \%$ saline; i.e. sham), or 6-OHDA $(2.5,5.0$ or $7.5 \mu \mathrm{g} / \mu \mathrm{l}$; Sigma, Zwijndrecht, The Netherlands) at the following coordinates relative to Bregma (in mm): AP 0.7, $\mathrm{ML}+/-2.8, \mathrm{DV}$ 5.0 and AP -0.4, ML +/-3.4, DV -5.0) (Paxinos and Watson, 1998), at $0.5 \mu \mathrm{l} / \mathrm{min}$. After surgery, the rats were given a 2-week recovery period before behavioural training was started. Data from all behavioural tests were acquired 3-4 weeks after surgery.

\section{Reaction time task}

Rats were trained to perform a reaction time (RT) task preoperatively. The motor performances of the rats were tested in operant chambers as described earlier (Blokland, 1998, Temel, et al., 2005). After training, the rats were tested for motor time performance. Post surgery the rats were trained again and were tested. The motor time was acquired before and after the injection of 6-OHDA or saline into the striatum. The average motor time (MT) was calculated over five trials. For each animal the increase in motor time and RT was calculated and expressed in percentages, as for the change in premature responses (PR).

Open field test

The open field was performed as described earlier (Temel, et al., 2006). Locomotor activity (distance moved and speed) was recorded using a camera and Ethovision tracking software (Ethovision ${ }^{\circledR}$, Noldus Information Technology, Wageningen, The Netherlands).

\section{CatWalk gait analysis}

Pre- and post- surgically the rats were trained to cross the walkway (Catwalk XT8.1, Noldus, The Netherlands), i.e. they were motivated by food deprivation $12 \mathrm{~h}$ before training and rewarded with a food pellet (Noyes Precision pellets PJPPP-0045; Sandown Chemical Ltd., Hampton, UK) after a complete and continuous crossing. Training continued until crossing the walkway met the criteria for acquisition (Hamers, et al., 2001). The following gait parameters were evaluated: speed, walking pattern and standing 
time, for more details we refer to Vlamings et al. 2007 (Vlamings, et al., 2007). For statistical analysis the data of 5 trials was averaged.

\section{Electrophysiology}

At the end of the behavioural experiments, rats were anesthetized with urethane (1.6 $\mathrm{g} / \mathrm{kg}$, i.p.) and fixed in a stereotactic frame (Stoelting model 51950, Stoelting Co, Wood Dale, USA). Body temperature was maintained at $37^{\circ} \mathrm{C}$ (DC Temperature controller 4190-8D, FHC Inc. Bowdoin, USA). A glass microelectrode (borosilicate glass capillaries [GC200F-10], Harvard apparatus LTD, Massachusetts, USA), with an impedance of \pm 10 $15 \mathrm{M} \Omega$, containing $4 \%$ Pontamine sky blue dye dissolved in $0.9 \% \mathrm{NaCl}$ was then lowered into the STN using a microdrive (Hydraulic probe drive chronic adaptor, 50-12-9 FHC Inc. Bowdoin, USA). Stereotactic coordinates in mm relative to Bregma were: AP -3.8, $\mathrm{ML}+$-2.5, DV -8.0 (Paxinos and Watson, 1998). The electrode was connected to an AlphaMap data acquisition system (AlphaOmega, Nazareth, Israel), allowing amplification, filtering for live view and recording the extracellular neuronal activity. At the end of each session, the recording site was marked by an electrophoretic injection of Pontamine sky blue using a $15 \mu \mathrm{A}$ negative current for $20 \mathrm{~min}$ (Accupulser signal generator and a high current isolator [A385] with a charger [A382], WPI Inc. Sarasota, USA). Neurons were only analysed if the recording site was histochemically confirmed to be in the STN (Fig 3a).

Raw data were analysed using Matlab (The MathWorks, Natick, MA). Spikes were detected based on a threshold that was defined as the standard deviation of the signalmedian multiplied by 3.5 (Dolan, et al., 2009). Classification of the spikes was based on the waveforms of the detected spikes. First, a principal component analysis was performed. Subsequently, the principal components were clustered using either K-means or the Gaussian mixture model and the expectation maximization algorithm. Only time intervals with a stable firing rate and neurons with a signal to noise ratio greater than three were considered.

A discharge density histogram (Kaneoke and Vitek, 1996) was developed to assess the firing pattern as previously described (Boraud, et al., 1998, Chetrit, et al., 2009). The mean firing frequency was calculated per group.

\section{Histology}

At the end of the electrophysiological recordings, rats were transcardially perfused and prepared for immunohisstochemistry. Tyrosine hydroxylase (TH) immunohistochemistry was carried out using a monoclonal mouse anti-TH (diluted 1:100, kindly supplied by Dr. C. Cuello, Canada) as primary antibody. Details of this staining have been described previously (Temel, et al., 2006). 
Stereological analysis

Stereological analysis was performed using a stereology workstation (CAST-GRIDComputer Assisted Stereological Toolbox, Olympus, Denmark). After exactly tracing the boundaries of the left and right substantia nigra pars compacta (SNc) 10x magnification, number of TH immunoreactive (THir) neurons was counted at 40x magnification using the Optical Fractionator (Schmitz and Hof, 2005). All neurons that were in focus in the unbiased virtual counting spaces throughout the delineated regions were counted. The number of neurons was estimated based on the number of counted neurons and the corresponding sampling probability. The total number of THir neurons in the left and right SNc was averaged per animal.

\section{Statistical analyses}

The data from the RT task, the open field, the electrophysiology and the data on the total number of THir cells in the SNc were analysed using a one-way ANOVA followed by a Least Significant Difference (LSD) post-hoc test. Electrophysiological differences in firing pattern were evaluated by a Chi-square test. The parameters of the Catwalk were analysed using a repeated measures one-way ANOVA followed by an LSD post-hoc test. All data were analysed using SPSS statistical software (SPSS version 15.0, IBM, Chicago, USA). $p<0.05$ was considered to be statistically significant. All data are presented as means and standard errors of means (SEM).

\section{Results}

Histology

No significant difference was found between the number of THir cells in the SNc of the left and right side of the brain, therefore we pooled the data. Compared to sham controls, the number of THir cells in the SNc was reduced by $20 \%$ in the $2.5 \mu \mathrm{g} / \mu \mathrm{l} 6-\mathrm{OHDA}$ group, by $42 \%$ in the $5 \mu \mathrm{g} / \mu \mathrm{l} 6-\mathrm{OHDA}$ group, and by $41 \%$ in the $7.5 \mu \mathrm{g} / \mu \mathrm{l} 6$-OHDA group (Fig. 1). In the 6-OHDA treatment groups, the $5 \mu \mathrm{g} / \mu \mathrm{l}$ and $7.5 \mu \mathrm{g} / \mu \mathrm{l} 6-\mathrm{OHDA}$ groups had significantly less THir cells than in the $2.5 \mu \mathrm{g} / \mu \mathrm{l}$ 6-OHDA group.

\section{Reaction time task}

Sham surgery did not affect MT, whereas all 6-OHDA groups showed a 20\% longer MT after surgery $(p<0.01$; Fig. 2a). The sham surgery and the 6-OHDA treatment had no effect on the RT and PR (Figs. 2b and c). 

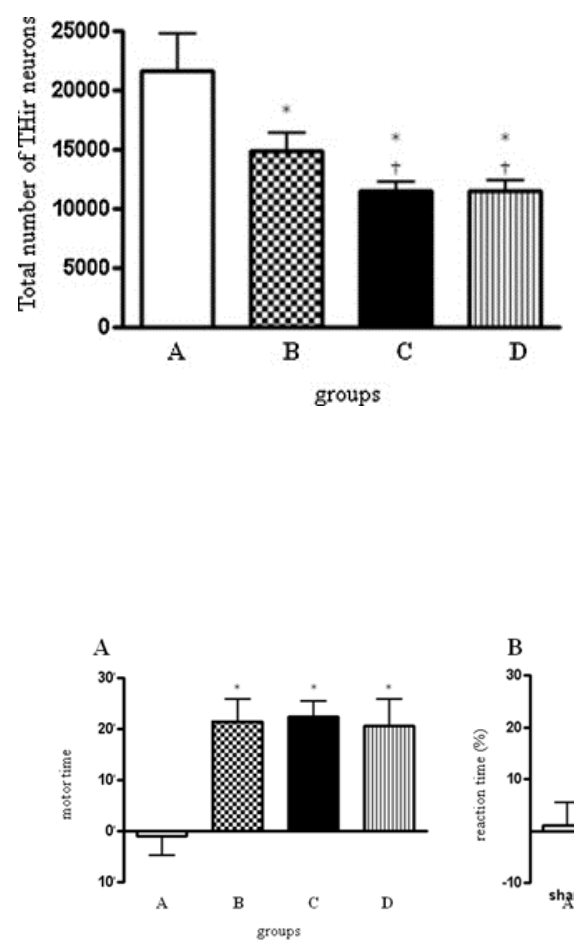
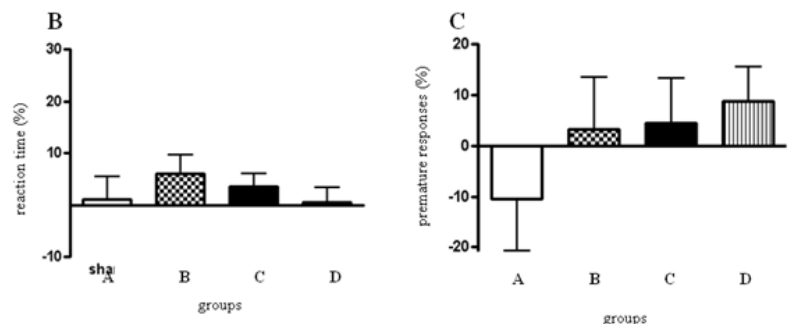

Fig. 2. (a) Mean changes in motor time compared to baseline (\%). *Groups differ significantly from sham group ( $p<0.05)$. (b) Mean changes in reaction time compared to baseline (\%). No significant differences were observed between groups ( $p>0.05$ ). (c) Mean changes in premature responses compared to base line (\%). No significant differences were observed between groups $(p>0.05)$. Data represent means and S.E.M. per group. Abbreviations are as follows: A: sham; B, C and D: 2.5, 5.0 and $7.5 \mu \mathrm{g} / \mu \mathrm{l} 6-\mathrm{OHDA}$, respectively.

\section{Open field}

Sham surgery and 6-OHDA treatment had no effect on speed and distance moved during a $10 \mathrm{~min}$ open field test. The measured speed (in $\mathrm{m} / \mathrm{min}$ ) in the sham, 2.5, 5.0 and $7.5 \mathrm{mg} / \mathrm{ml}$ 6-OHDA groups was respectively 11.1 (2.2), 12.0 (1.6), 10.4 (1.7), 10.3 (1.1) ( $>0.05$, data not shown). The distance moved (in $\mathrm{cm}$ ) was respectively 6641 (503), 7180 (365), 6235 (394), 6199 (250) (p>0.05, Fig. 3a and b). 
A

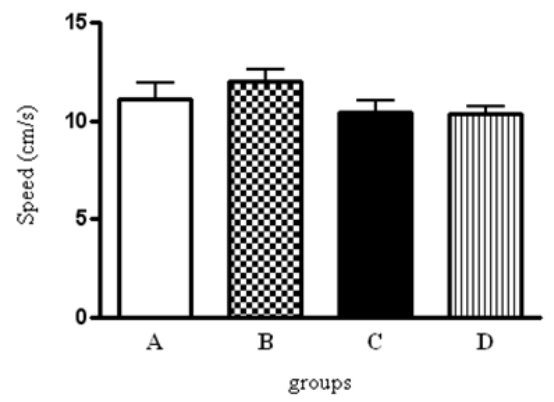

B

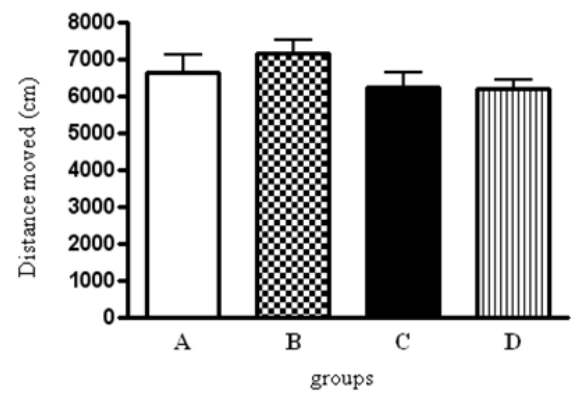

Fig. 3. (a) Mean speed in the open field during 10 minutes $(\mathrm{cm} / \mathrm{s})$ No significant differences were observed between groups. (b) Mean distance moved $(\mathrm{cm})$ in the open field during 10 minutes of testing. No significant differences were observed between groups. Data represent means and S.E.M. per group. Abbreviations are as follows: A: sham; B, C and D: respectively 2.5, 5.0 and $7.5 \mu \mathrm{g} / \mu \mathrm{l} 6-\mathrm{OHDA}$.

\section{Catwalk gait analysis}

Surgery resulted in a significantly reduced speed (in $\mathrm{mm} / \mathrm{s}$ ), a reduced standing time and a changed walking pattern. However, there was no significant difference between the sham and 6-OHDA treatment groups for these parameters. The measured speed (in $\mathrm{mm} / \mathrm{s}$ ) and standing time (in s) in the sham, 2.5, 5.0 and $7.5 \mathrm{mg} / \mathrm{ml}$ 6-OHDA groups after surgery were respectively: 646 (56), 595 (70), 624 (22), 540 (85) (p>0.05) and 0.10 (0.01), 0.11 (0.01), $0.11(<0.00), 0.13(0.02)$ ( $p>0.05)$. The step pattern, either cruciate or alternate, was respectively for 70 (17), 88 (11), 72 (16) and 77 (10) percent alternated ( $p>0.05$, Fig 4$)$.
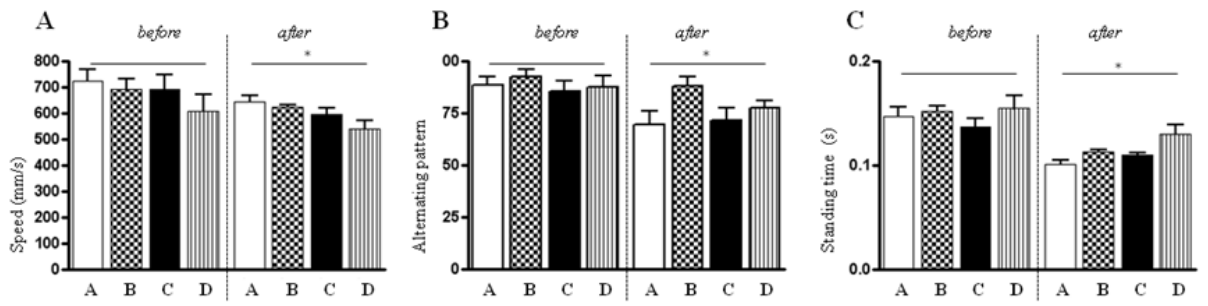

Fig. 4. (a) Speed on the catwalk (mm/s). No significant differences were found between groups $(p>0.05) .{ }^{*}$ In general speed was significantly reduced in all groups after surgery compared to baseline $(p<0.05)$. (b) Percentage the rats show an alternate walking pattern (\%). There were no significant differences seen between groups $(p>0.05)$, * but after surgery the percentage of alternate movements was decreased $(p<0.05)$. (c) Standing time on the catwalk (s). No significant differences were found between groups ( $p>0.05) .{ }^{*}$ In general standing time was significantly reduced in all groups after surgery compared to baseline $(p<0.05)$. Data represent means and S.E.M. per group. Abbreviations are as follows: A: sham; B, C and D: respectively 2.5, 5.0 and $7.5 \mu \mathrm{g} / \mu \mathrm{l}$ 6-OHDA. 


\section{Electrophysiology}

In total 338 neurons were recorded, STN neurons from sham animals showed $7.8 \pm 0.9$ spikes per second, which was similar to that of neurons from 6-OHDA treated animals (Fig. 3b). All groups displayed neurons with a regular, an irregular and a bursty type firing pattern (Fig. 3c). Whereas sham animals displayed mostly regular type firing neurons (54\%) and only 19\% bursty type neurons, 6-OHDA treated animals displayed a minor amount of regular type firing neurons (32-38\%) and mostly, i.e. 41-49\% bursty type neurons $(p<0.05)$. However, the distribution of regular, irregular and bursty type firing neurons did not differ between the different 6-OHDA treatment groups.
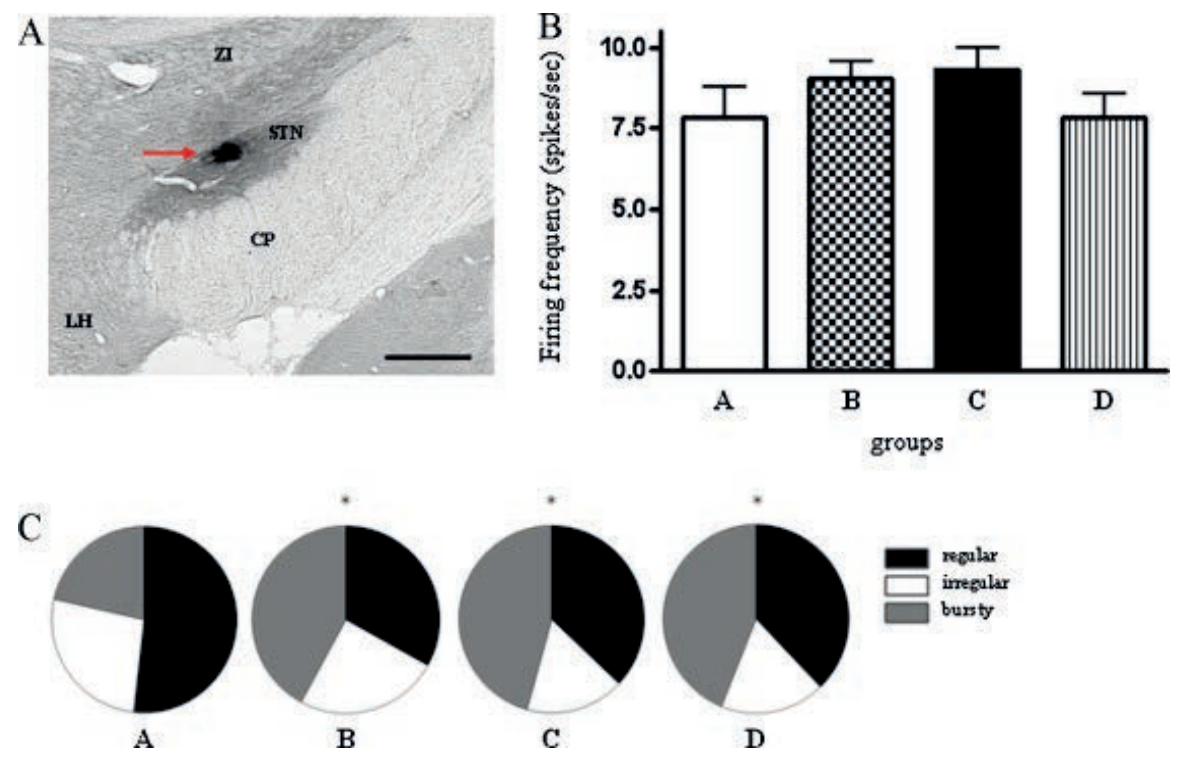

Fig. 3. (a) Representative low-power photomicrograph of a coronal brain section showing the subthalamic nucleus of a subject with an ionthophoretic injection (red arrow) at the site of the last recorded neuron. Scale bar: $500 \mu \mathrm{m}$. (b) Mean firing frequencies are displayed in spikes per seconds for sham and 6-OHDA groups. No significant differences were found $(p>0.05)$. Data represent means and S.E.M. per group. (c) Distribution of the firing pattern (\%). In group A approximately 54\% of the cells is regular, $27 \%$ irregular and $19 \%$ bursty. In the groups B, C and D the percentage of regular cells decreases to $32-38 \%$ and the number of bursty cells increases to $41-49 \% . \chi^{2} p<0.05$. Abbreviations are as follows: A: sham; B, C and D: $2.5,5.0$ and 7.5 6-OHDA, respectively; CP: cerebral peduncle; STN: subthalamic nucleus, ZI: zona incerta; LH: lateral hypothalamus.

\section{Discussion}

In this study we investigated the link between the level of DA depletion, motor symptoms and STN burst activity in rats with different levels of dopaminergic denervation. Aside from a slower motor response in the reaction time task, animals with a mild lesion (i.e. $20 \%$ loss of THir cells in the SNc) displayed no gross locomotor abnormalities. How- 
ever, the firing pattern of their STN neurons shifted from a more regular firing type to a burst mode. Animals with a substantial lesion (i.e. $40 \%$ loss of THir cells in the SNc), showing the same slowing in the motor response, had a similar shift in the firing pattern. Thus, the electrophysiological properties of the STN already shifted to a bursty phenotype due to a mild DA depletion before severe motor symptoms were present.

The question arises if more severe lesioned animals, with more motor disabilities would have had a dose-dependent change in STN burst activity. Unexpectedly, the 7.5 $\mu \mathrm{g} 6$-OHDA treated group had a similar level of loss of THir cells as the $5 \mu \mathrm{g} 6$-OHDA group (Deumens, et al., 2002). Interestingly in literature, the amount of STN bursty neurons found in this study was similar to the number of bursty cells found in a study with a partial and severe 6-OHDA model (Breit, et al., 2007). On the other hand, one 6OHDA study showed a very high percentage of bursty neurons (90\%) (Walters, et al., 2007), while other studies with severe DA lesions report comparable percentages of bursty neurons as found in this study (Hollerman and Grace, 1992, Ni, et al., 2001).

In PD patients symptoms do not appear before more than 70 to $80 \%$ of the DA neurons in the SNc are lost. Such a late manifestation of symptoms must be due to the existence of compensatory mechanisms (Zigmond, et al., 1990). Also in this rodent study no gross motor changes were observed with relatively mild lesions (20-40\% loss DA neurons). However this study evidenced that a small loss of DA neurons in the SNC gave rise to a bursty firing pattern in the STN of the rat. The abnormal bursty firing pattern in DA depleted animals, generated by deep urethane anaesthesia, is in phase with the cortical slow wave activity $(\sim 1 \mathrm{~Hz})$ (Magill, et al., 2001). The shift of STN neuronal activity from a regular to a bursty phenotype in mild DA depleted animals may be a direct result of DA input from the SNc on the STN. The STN contains a high number of D5 receptors for dopamine (Svenningsson and Le Moine, 2002), which play a pivotal role in the control of the $\mathrm{Ca}^{2+}$ conductance necessary for neurons to express burst firing (Baufreton, et al., 2003). The early occurrence of a bursty phenotype might also be mediated by the indirect pathway via the globus pallidus (GP) (Breit, et al., 2007). Baufreton and Bevan 2008 (Baufreton and Bevan, 2008) described that the STN firing pattern is influenced by the pre-synaptic D2 receptors on the GP terminals into the STN.

A provocative hypothesis is that hyperactivity of the STN is compensatory mechanism to drive the remaining SNc neurons to enhance the dopamine release through the nigrostriatal pathway (Bezard, et al., 1999). Thus the early increase in STN burst activity, which remains with disease progression, might be a compensatory mechanism to maintain a DA homeostasis within the basal ganglia. To investigate this hypothesis, further studies are needed to assess the relationship between changes in STN and SNc neuronal firing properties and DA release in the striatum. 


\section{Acknowledgements}

The authors gratefully acknowledge the support of the BrainGain Smart Mix Programme of the Netherlands Ministry of Economic Affairs and the Netherlands Ministry of Education, Culture and Science (grant no.: SSM06011) and the Netherlands Organisation for Scientific Research (Veni grant, no.: 016.096.032). 


\section{References}

1. Afsharpour, S., 1985. Topographical projections of the cerebral cortex to the subthalamic nucleus. J Comp Neurol 236, 14-28.

2. Albin, R. L., Young, A. B., and Penney, J. B., 1989. The functional anatomy of basal ganglia disorders. Trends Neurosci 12, 366-375.

3. Baufreton, J., and Bevan, M. D., 2008. D2-like dopamine receptor-mediated modulation of activitydependent plasticity at GABAergic synapses in the subthalamic nucleus. J Physiol 586, 2121-2142.

4. Baufreton, J., Garret, M., Rivera, A., de la Calle, A., Gonon, F., Dufy, B., Bioulac, B., and Taupignon, A., 2003. D5 (not D1) dopamine receptors potentiate burst-firing in neurons of the subthalamic nucleus by modulating an L-type calcium conductance. J Neurosci 23, 816-825.

5. Benabid, A. L., Pollak, P., Gross, C., Hoffmann, D., Benazzouz, A., Gao, D. M., Laurent, A., Gentil, M., and Perret, J., 1994. Acute and long-term effects of subthalamic nucleus stimulation in Parkinson's disease. Stereotact Funct Neurosurg 62, 76-84.

6. Benazzouz, A., Breit, S., Koudsie, A., Pollak, P., Krack, P., and Benabid, A. L., 2002. Intraoperative microrecordings of the subthalamic nucleus in Parkinson's disease. Mov Disord 17 Suppl 3, S145-149.

7. Benazzouz, A., Gross, C., Dupont, J., and Bioulac, B., 1992. MPTP induced hemiparkinsonism in monkeys: behavioral, mechanographic, electromyographic and immunohistochemical studies. Exp Brain Res 90, 116-120.

8. Bergman, H., Wichmann, T., Karmon, B., and DeLong, M. R., 1994. The primate subthalamic nucleus. II. Neuronal activity in the MPTP model of parkinsonism. J Neurophysiol 72, 507-520.

9. Bezard, E., Boraud, T., Bioulac, B., and Gross, C. E., 1999. Involvement of the subthalamic nucleus in glutamatergic compensatory mechanisms. Eur J Neurosci 11, 2167-2170.

10. Blokland, A., 1998. Reaction time responding in rats. Neurosci Biobehav Rev 22, 847-864.

11. Boraud, T., Bezard, E., Guehl, D., Bioulac, B., and Gross, C., 1998. Effects of L-DOPA on neuronal activity of the globus pallidus externalis (GPe) and globus pallidus internalis (GPi) in the MPTP-treated monkey. Brain Res 787, 157-160.

12. Breit, S., Bouali-Benazzouz, R., Popa, R. C., Gasser, T., Benabid, A. L., and Benazzouz, A., 2007. Effects of 6-hydroxydopamine-induced severe or partial lesion of the nigrostriatal pathway on the neuronal activity of pallido-subthalamic network in the rat. Exp Neurol 205, 36-47.

13. Carpenter, M. B., Carleton, S. C., Keller, J. T., and Conte, P., 1981. Connections of the subthalamic nucleus in the monkey. Brain Res 224, 1-29.

14. Chetrit, J., Ballion, B., Laquitaine, S., Belujon, P., Morin, S., Taupignon, A., Bioulac, B., Gross, C. E., and Benazzouz, A., 2009. Involvement of Basal Ganglia network in motor disabilities induced by typical antipsychotics. PLoS One 4, e6208.

15. Deumens, R., Blokland, A., and Prickaerts, J., 2002. Modeling Parkinson's disease in rats: an evaluation of 6-OHDA lesions of the nigrostriatal pathway. Exp Neurol 175, 303-317.

16. Dolan, K., Martens, H. C., Schuurman, P. R., and Bour, L. J., 2009. Automatic noise-level detection for extra-cellular micro-electrode recordings. Med Biol Eng Comput 47, 791-800.

17. Hameleers, R., Blokland, A., Steinbusch, H. W., Visser-Vandewalle, V., and Temel, Y., 2007. Hypomobility after DOI administration can be reversed by subthalamic nucleus deep brain stimulation. Behav Brain Res 185, 65-67.

18. Hamers, F. P., Lankhorst, A. J., van Laar, T. J., Veldhuis, W. B., and Gispen, W. H., 2001. Automated quantitative gait analysis during overground locomotion in the rat: its application to spinal cord contusion and transection injuries. J Neurotrauma 18, 187-201.

19. Hollerman, J. R., and Grace, A. A., 1992. Subthalamic nucleus cell firing in the 6-OHDA-treated rat: basal activity and response to haloperidol. Brain Res 590, 291-299.

20. Hutchison, W. D., Allan, R. J., Opitz, H., Levy, R., Dostrovsky, J. O., Lang, A. E., and Lozano, A. M., 1998. Neurophysiological identification of the subthalamic nucleus in surgery for Parkinson's disease. Ann Neurol 44, 622-628. 
21. Kaneoke, Y., and Vitek, J. L., 1996. Burst and oscillation as disparate neuronal properties. J Neurosci Methods 68, 211-223.

22. Krack, P., Batir, A., Van Blercom, N., Chabardes, S., Fraix, V., Ardouin, C., Koudsie, A., Limousin, P. D., Benazzouz, A., LeBas, J. F., Benabid, A. L., and Pollak, P., 2003. Five-year follow-up of bilateral stimulation of the subthalamic nucleus in advanced Parkinson's disease. N Engl J Med 349, 1925-1934.

23. Magill, P. J., Bolam, J. P., and Bevan, M. D., 2001. Dopamine regulates the impact of the cerebral cortex on the subthalamic nucleus-globus pallidus network. Neuroscience 106, 313-330.

24. Monakow, K. H., Akert, K., and Kunzle, H., 1978. Projections of the precentral motor cortex and other cortical areas of the frontal lobe to the subthalamic nucleus in the monkey. Exp Brain Res 33, 395-403.

25. Nakanishi, H., Kita, H., and Kitai, S. T., 1987. Electrical membrane properties of rat subthalamic neurons in an in vitro slice preparation. Brain Res 437, 35-44.

26. Ni, Z. G., Bouali-Benazzouz, R., Gao, D. M., Benabid, A. L., and Benazzouz, A., 2001. Time-course of changes in firing rates and firing patterns of subthalamic nucleus neuronal activity after 6-OHDA-induced dopamine depletion in rats. Brain Res 899, 142-147.

27. Overton, P. G., and Greenfield, S. A., 1995. Determinants of neuronal firing pattern in the guinea-pig subthalamic nucleus: an in vivo and in vitro comparison. J Neural Transm Park Dis Dement Sect 10, 4154.

28. Paxinos, G., and Watson, C., 1998. The Rat Brain in Stereotaxic Coordinates. Academic Press, New York.

29. Ricardo, J. A., 1980. Efferent connections of the subthalamic region in the rat. I. The subthalamic nucleus of Luys. Brain Res 202, 257-271.

30. Rodriguez, M. C., Guridi, O. J., Alvarez, L., Mewes, K., Macias, R., Vitek, J., DeLong, M. R., and Obeso, J. A., 1998. The subthalamic nucleus and tremor in Parkinson's disease. Mov Disord 13 Suppl 3, 111-118.

31. Schmitz, C., and Hof, P. R., 2005. Design-based stereology in neuroscience. Neuroscience 130, 813-831.

32. Smith, Y., Bevan, M. D., Shink, E., and Bolam, J. P., 1998. Microcircuitry of the direct and indirect pathways of the basal ganglia. Neuroscience 86, 353-387.

33. Svenningsson, P., and Le Moine, C., 2002. Dopamine D1/5 receptor stimulation induces c-fos expression in the subthalamic nucleus: possible involvement of local D5 receptors. Eur J Neurosci 15, 133-142.

34. Tan, S., Vlamings, R., Lim, L., Sesia, T., Janssen, M. L., Steinbusch, H. W., Visser-Vandewalle, V., and Temel, Y., 2010. Experimental deep brain stimulation in animal models. Neurosurgery 67, 1073-1079; discussion 1080.

35. Temel, Y., Kessels, A., Tan, S., Topdag, A., Boon, P., and Visser-Vandewalle, V., 2006. Behavioural changes after bilateral subthalamic stimulation in advanced Parkinson disease: a systematic review. Parkinsonism Relat Disord 12, 265-272.

36. Temel, Y., Visser-Vandewalle, V., Aendekerk, B., Rutten, B., Tan, S., Scholtissen, B., Schmitz, C., Blokland, A., and Steinbusch, H. W., 2005. Acute and separate modulation of motor and cognitive performance in parkinsonian rats by bilateral stimulation of the subthalamic nucleus. Exp Neurol 193, 43-52.

37. Temel, Y., Visser-Vandewalle, V., Kaplan, S., Kozan, R., Daemen, M. A., Blokland, A., Schmitz, C., and Steinbusch, H. W., 2006. Protection of nigral cell death by bilateral subthalamic nucleus stimulation. Brain Res 1120, 100-105.

38. Visser-Vandewalle, V., van der Linden, C., Temel, Y., Celik, H., Ackermans, L., Spincemaille, G., and Caemaert, J., 2005. Long-term effects of bilateral subthalamic nucleus stimulation in advanced Parkinson disease: a four year follow-up study. Parkinsonism Relat Disord 11, 157-165.

39. Vlamings, R., Visser-Vandewalle, V., Koopmans, G., Joosten, E. A., Kozan, R., Kaplan, S., Steinbusch, H. W., and Temel, Y., 2007. High frequency stimulation of the subthalamic nucleus improves speed of locomotion but impairs forelimb movement in Parkinsonian rats. Neuroscience 148, 815-823.

40. Walters, J. R., Hu, D., Itoga, C. A., Parr-Brownlie, L. C., and Bergstrom, D. A., 2007. Phase relationships support a role for coordinated activity in the indirect pathway in organizing slow oscillations in basal ganglia output after loss of dopamine. Neuroscience 144, 762-776.

41. Wichmann, T., Bergman, H., and DeLong, M. R., 1994. The primate subthalamic nucleus. I. Functional properties in intact animals. J Neurophysiol 72, 494-506. 
CHAPTER 9

42. Zigmond, M. J., Abercrombie, E. D., Berger, T. W., Grace, A. A., and Stricker, E. M., 1990. Compensations after lesions of central dopaminergic neurons: some clinical and basic implications. Trends Neurosci 13, 290-296. 
DISCUSSION 
In the present thesis, we first showed the short- and long-term outcome of STN DBS in PD patients. In general STN DBS has a good effect on the motor symptoms, on the other hand a substantial number of patients (42\%) developed psychiatric symptoms, such as increased impulsive behavior, depressed mood and hypomanic behavior. The amount of reported neuropsychiatric side-effects decreased in the last decennia (Odekerken, et al., 2013). This is probably due to the improved imaging and targeting techniques, and a better patient selection. On the long term the positive effect of STN DBS on specific symptoms diminishes due to disease progression. Axial symptoms become more pronounced, which are unresponsive to L-DOPA and STN DBS treatment. Subsequently, we investigated the functional anatomical connectivity of the STN with the DRN to explain the STN DBS induced side effects. In an animal model of PD we showed that HFS of the STN induced depressive like behavior. An increase in c-Fos in mainly GABA-ergic neurons in the DRN, and decreased cytochrome $C$ oxidase activity in this region was induced by HFS of the STN. HFS of the STN also increased c-Fos in the lateral habenula, medial prefrontal cortex. These data implicate that the STN is indirectly connected to the serotonergic dorsal raphe nucleus. In addition, we provided a systematic review of the functional topography of the STN and its afferent and efferent connections. In line with, findings of this review we showed that STN is strongly involved in limbic processes and functionally connected to limbic brain regions. Subsequently, in an animal model we showed that motor and non-motor pathways are not fully segregated, but partially interconnected in the STN. Thereafter, we aimed to improve targeting of the STN motor region in a clinical study. Unfortunately, we were not able to provide a clear marker for the STN motor region. The results from single-unit and LFP recordings were promising, though caution is needed when repetitive, subdural cortical stimulation is applied. Next we showed that the Catwalk is a reproducible tool to assess gait in a bilateral 6-OHDA rat model of PD. Following this study, we studied the relevance of STN burst activity in relation to motor and non-motor symptoms. We showed that STN burst activity is present when only minor behavioral symptoms are present. The results from this study implicate that STN burst activity might be a compensatory mechanism to instigate the remaining dopaminergic SNc neurons to keep the amount of available dopamine in the striatum at a sufficient level. Here, we will discuss the results from the studies performed, review the serious unexpected side-effects, elaborate on the clinical implications of our findings and give directions for future research.

As stated earlier in this thesis, the human STN is classically subdivided into a dorsolateral sensorimotor area, a ventral associative and limbic medial tip (Hamani, et al., 2004, Parent and Smith, 1987, Temel, et al., 2005). In line with this view, anatomical studies in animals indeed show that projections from cortical motor areas are most dense in the dorsolateral part of the STN. Afferent connections of the STN with associative and limbic brain regions are mainly found in the ventro-medial parts of the STN. 
The topographical organization of the cortico-subthalamic projections in rodents and non-human primates overlaps with the topographical organization of the other afferent and efferent connections of the STN. The dorsolateral, motor STN receives input from the lateral part of the parafascicular thalamic nucleus, which also projects to the lateral part of the caudate putamen (CPu). The lateral CPu indirectly projects to the lateral STN via the lateral part of the GPe. The medial, limbic part of the STN receives its input from the most medial part of the parafascicular thalamic nucleus, which also projects to the nucleus accumbens (NAc) and the medial CPu. The NAc and the medial CPu project indirectly to the medial STN via respectively the lateral part of the subcommisural ventral pallidum and the medial part of the GPe. The NAc, in turn, receives input from limbic, prefrontal areas. Also the efferent output of the STN to the GPe, GPi and the ventral pallidum overlaps. The lateral STN mainly projects to the lateral GPe and GPi, whereas the medial part projects to the ventral pallidum and the medial GPe and GPi. (Berendse and Groenewegen, 1990, Karachi, et al., 2005, Kita and Kitai, 1987, Parent and Hazrati, 1995). In agreement with the anatomical tracing studies, electrophysiological studies have also shown a crude functional subdivision. However, in rats a significant number of STN neurons respond to stimulation of motor and non-motor cortical regions as we have also shown in chapter 5 . This finding doesn't support the theory of parallel corticobasal ganglia pathways, but proofs that the cortico-subthalamic pathways are partially integrated. This neurophysiological finding is supported by the existence of a relationship between cognitive and emotional processes and motor behavior (Bloem, et al., 2001, Giladi, et al., 2005, Hausdorff, et al., 2006) and by the fact that neurons located in the effective dorso-lateral region of the STN for DBS in PD patients respond to emotional stimuli (Fonoff, et al., 2012). This finding is also reflected by the overlap in functional subdivisions in anatomical studies (Kita, et al., 2014). To our knowledge, no electrophysiological studies in primates or humans are available which investigated whether single STN neurons receive convergent input from motor, associative and/or limbic cortical areas as we have shown in the rodent. A recent DTI study in humans sub-parcelated the STN in an anterio-medial limbic, middle associative and posterior-lateral motor area (Lambert, et al., 2012). Connectivity between the STN and motor and non-motor regions was often not confined to one of the STN functional subterritories. Important to mention is that the sub-parcellation was based on STN connectivity with subcortical structures and could not be based on cortico-subthalamic connections. The majority of connecting cortical regions were found to receive contributions from all STN subregions, but with different proportions of each type. Interestingly, the limbic connections were in general more prominent in the left hemisphere.

Since the beginning of STN DBS for the treatment of Parkinson's disease this surgical therapy proved to be effective. Also on the long term a preserved effect on the core motor symptoms was seen. Several long-term follow-up studies, up to 10 years after 
DBS implantation, showed sustained effect on tremor and rigidity. Unfortunately, axial symptoms did not seem to respond well to STN DBS. The increase in time on the UDPRS motor scale is probably due to further disease progression. Also an increase in disability and cognitive impairment due to disease progression was seen in these patients (Castrioto, et al., 2011, Fasano, et al., 2010, Zangaglia, et al., 2012, Zibetti, et al., 2011)_ENREF_12. In our case series, we reported similar outcome after 10 years (chapter 1). It can therefore be concluded that STN DBS has a sustained effect on PD motor symptoms. To be able to also improve the axial symptoms and thereby reduce falling and quality of life other treatments are necessary. Also investigation on stimulation of brainstem structures, such as the pedunculo-pontine nucleus and the spinal cord to improve gait need further attention. In chapter 8 we validated a bilateral 6-OHDA rat model for PD to study gait. This animal model is currently used to further asses the gait dysfunction. Special emphasis in this research is given on the influence of the different mono-aminergic systems and the role of the brainstem nuclei and cerebellum in gait dysfunction in PD.

Unfortunately, STN DBS has not only positive effects on the motor symptoms, but also affects mood and cognition in a substantial number of patients (Berney, et al., 2002, Piasecki and Jefferson, 2004, Saint-Cyr, et al., 2000, Smeding, et al., 2006, Temel, et al., 2006, Witt, et al., 2008). Still, the unwanted psychiatric side effects of STN DBS may overshadow the positive effects on the motor symptoms and become a major burden for both the patient and relatives. The challenge remains to obtain good therapeutic effects and to prevent the occurrence of undesired psychiatric side effects. It should be noticed though that in a recent randomized controlled trial, in the secondary outcome measures, the psychiatric and cognitive side-effects between PD patients treated with STN DBS or globus pallidus internus (GPi) stimulation were the same in both groups. But, the STN treated patients had a favorable motor on the motor symptoms (Odekerken, et al., 2013). The undesired psychiatric side effects of STN DBS are thought to be caused by stimulation of the non-motor parts of the STN (Temel, et al., 2005). These psychiatric effects are associated with alterations in the serotonergic system. High frequency stimulation (HFS) of the STN inhibited dorsal raphne nucleus (DRN) neuronal firing and a reduced the release of serotonin (5-hydroxytryptamine, 5-HT) in the rat (Navailles, et al., 2010, Tan, et al., 2010, Temel, et al., 2007). The occurrence of these side-effects in the individual patient might be caused by a predisposed vulnerability of the 5-HT system. One might also question whether the "side-effects" of STN DBS on mood and cognition in PD patients could also be converted into a treatment for intractable psychiatric diseases. Currently trials are being conducted to assess the possibility of the STN as a surgical target for the treatment of psychiatric disorders, such as obsessive compulsive disorder and depression. It might also be well possible that other 
nuclei connected to the STN might be a better target for the surgical treatment of psychiatric diseases.

To overcome the DBS induces behavioural side-effects, we attempted to target the motor part of the STN by identifying the motor part by electrophysiological recordings of cortical evoked potentials. Unfortunately, this technique proofed not to be successful and serious adverse events occurred. Seizures were induced by subdural cortical stimulation. As discussed in chapter 6, this was probably due to monophasic application of the pulses and a too high current density. The question remains whether the monosynaptic cortico-subthalamic pathway could be a future roadmap to target the motor region of the STN in order to more selectively implant DBS electrodes for the treatment PD patients. Imaging techniques, like DTI, have been developed rapidly in the last years. Thus far no STN subregions could delineated based on the cortico-subthalamic connectivity by DTI studies in humans. In the near future it might however be possible to point out the motor area within the STN by visualizing the cortico-subthalamic pathway using DTI. Although, in our hands electrophysiological approach was not successful, with advanced neurophysiological techniques this might be technically achievable and safe. But another question needs to be answered first. Is it possible to selectively stimulate the motor region of the STN? In chapter 5 we have shown that the motor-and non-motor pathways are not fully segregated. On the contrary, a large overlap is seen between motor and non-motor regions in the rodent STN. In humans, the segregation is probably more robust. If the interconnectivity of cortico-subthalamic pathways however is as strong in humans as in rats, then these side effects cannot be avoided. With the currently available techniques it cannot be avoided that neurons, which are placed within the neuronal network involved in limbic and cognitive processes, are affected by the applied current. To further define the area which is stimulated, new probes are being developed to steer the current applied. Thus, technically many steps can and still could be made to improve STN DBS. Another aspect of the occurrence of side-effects which needs attention is the patient selection. Hypothetically, some patients have a predisposed higher risk to develop behavioural side-effects which are not directly related to the DBS lead placement, but are caused by the vulnerability of the serotonergic system in the individual patient. It is therefore necessary to further investigate the role of the 5HT system in PD in general, and more specifically to the changes before and after the application of STN DBS to understand the occurrence of these side-effects. Moreover, in the future in might be possible by neuropsychological assessments to screen and exclude patients which are at risk for the development of unwanted neuropsychiatric sideeffects. 


\section{Final conclusions}

Our findings underline the fact that the STN possesses a key position in the cortico-basal-ganglia-thalamo-cortical network. The STN is strongly involved in motor, cognitive and limbic processes. A strict tripartite of STN subdivision can however not be made. At the single neuron level, cognitive, limbic and motor processes are being integrated.

From a clinical point of view, STN DBS is an effective therapy to treat PD symptoms, though further reduction in psychiatric side-effects remains a challenge. Improvement of targeting by using enhanced imaging techniques, improved electrophysiological recordings, and better stimulation electrodes might allow us to reduce undesired neuropsychiatric side-effects and enhance the therapeutic benefit. To improve axial symptoms in PD patients the focus for DBS treatment should be on the brainstem and cerebellar nuclei, and the influence of the non-dopaminergic mono-amines on gait needs further assessment. 


\section{References}

1. Berendse, H. W., and Groenewegen, H. J., 1990. Organization of the thalamostriatal projections in the rat, with special emphasis on the ventral striatum. J Comp Neurol 299, 187-228.

2. Berney, A., Vingerhoets, F., Perrin, A., Guex, P., Villemure, J. G., Burkhard, P. R., Benkelfat, C., and Ghika, J., 2002. Effect on mood of subthalamic DBS for Parkinson's disease: a consecutive series of 24 patients. Neurology 59, 1427-1429.

3. Bloem, B. R., Valkenburg, V. V., Slabbekoorn, M., and van Dijk, J. G., 2001. The multiple tasks test. Strategies in Parkinson's disease. Experimental brain research 137, 478-486.

4. Castrioto, A., Lozano, A. M., Poon, Y. Y., Lang, A. E., Fallis, M., and Moro, E., 2011. Ten-year outcome of subthalamic stimulation in Parkinson disease: a blinded evaluation. Arch Neurol 68, 1550-1556.

5. Fasano, A., Romito, L. M., Daniele, A., Piano, C., Zinno, M., Bentivoglio, A. R., and Albanese, A., 2010. Motor and cognitive outcome in patients with Parkinson's disease 8 years after subthalamic implants. Brain : a journal of neurology 133, 2664-2676.

6. Fonoff, E. T., Edgar, M., Sameshima, K., Teixeira, M. J., and Nicolelis, M. A. L., 2012. Functional convergence of parallel circuits within subthalamic nucleus: Intra-operative multichannel-microelectrode recording study during emotional, cognitive and motor tasks., International Congress of Parkinson's Disease and Movement Disorders Movement Disorders, Dublin, Ireland, pp. S.205.

7. Giladi, N., Herman, T., Reider, G., II, Gurevich, T., and Hausdorff, J. M., 2005. Clinical characteristics of elderly patients with a cautious gait of unknown origin. J Neurol 252, 300-306.

8. Hamani, C., Saint-Cyr, J. A., Fraser, J., Kaplitt, M., and Lozano, A. M., 2004. The subthalamic nucleus in the context of movement disorders. Brain 127, 4-20.

9. Hausdorff, J. M., Doniger, G. M., Springer, S., Yogev, G., Simon, E. S., and Giladi, N., 2006. A common cognitive profile in elderly fallers and in patients with Parkinson's disease: the prominence of impaired executive function and attention. Experimental aging research 32, 411-429.

10. Karachi, C., Yelnik, J., Tande, D., Tremblay, L., Hirsch, E. C., and Francois, C., 2005. The pallidosubthalamic projection: an anatomical substrate for nonmotor functions of the subthalamic nucleus in primates. Mov Disord 20, 172-180.

11. Kita, H., and Kitai, S. T., 1987. Efferent projections of the subthalamic nucleus in the rat: light and electron microscopic analysis with the PHA-L method. J Comp Neurol 260, 435-452.

12. Kita, T., Osten, P., and Kita, H., 2014. Rat subthalamic nucleus and zona incerta share extensively overlapped representations of cortical functional territories. J Comp Neurol 522, 4043-4056.

13. Lambert, C., Zrinzo, L., Nagy, Z., Lutti, A., Hariz, M., Foltynie, T., Draganski, B., Ashburner, J., and Frackowiak, R., 2012. Confirmation of functional zones within the human subthalamic nucleus: Patterns of connectivity and sub-parcellation using diffusion weighted imaging. Neuroimage 60, 83-94.

14. Navailles, S., Benazzouz, A., Bioulac, B., Gross, C., and De Deurwaerdere, P., 2010. High-frequency stimulation of the subthalamic nucleus and L-3,4-dihydroxyphenylalanine inhibit in vivo serotonin release in the prefrontal cortex and hippocampus in a rat model of Parkinson's disease. J Neurosci 30, 2356-2364.

15. Odekerken, V. J., van Laar, T., Staal, M. J., Mosch, A., Hoffmann, C. F., Nijssen, P. C., Beute, G. N., van Vugt, J. P., Lenders, M. W., Contarino, M. F., Mink, M. S., Bour, L. J., van den Munckhof, P., Schmand, B. A., de Haan, R. J., Schuurman, P. R., and de Bie, R. M., 2013. Subthalamic nucleus versus globus pallidus bilateral deep brain stimulation for advanced Parkinson's disease (NSTAPS study): a randomised controlled trial. Lancet Neurol 12, 37-44.

16. Parent, A., and Hazrati, L. N., 1995. Functional anatomy of the basal ganglia. II. The place of subthalamic nucleus and external pallidum in basal ganglia circuitry. Brain Res Brain Res Rev 20, 128-154.

17. Parent, A., and Smith, Y., 1987. Organization of efferent projections of the subthalamic nucleus in the squirrel monkey as revealed by retrograde labeling methods. Brain Res 436, 296-310.

18. Piasecki, S. D., and Jefferson, J. W., 2004. Psychiatric complications of deep brain stimulation for Parkinson's disease. J Clin Psychiatry 65, 845-849. 
19. Saint-Cyr, J. A., Trepanier, L. L., Kumar, R., Lozano, A. M., and Lang, A. E., 2000. Neuropsychological consequences of chronic bilateral stimulation of the subthalamic nucleus in Parkinson's disease. Brain 123 ( Pt 10), 2091-2108.

20. Smeding, H. M., Speelman, J. D., Koning-Haanstra, M., Schuurman, P. R., Nijssen, P., van Laar, T., and Schmand, B., 2006. Neuropsychological effects of bilateral STN stimulation in Parkinson disease: a controlled study. Neurology 66, 1830-1836.

21. Tan, S. K. H., Hartung, H., Temel, Y., and Sharp, T., High frequency stimulation of the subthalamic nucleus inhibts the firing of juxtacellular labelled 5-HT-containing neurons and decreases 5-HT release in vivo., Society for Neuroscience, San Diego, 2010.

22. Temel, Y., Blokland, A., Steinbusch, H. W., and Visser-Vandewalle, V., 2005. The functional role of the subthalamic nucleus in cognitive and limbic circuits. Prog Neurobiol 76, 393-413.

23. Temel, Y., Boothman, L. J., Blokland, A., Magill, P. J., Steinbusch, H. W., Visser-Vandewalle, V., and Sharp, T., 2007. Inhibition of 5-HT neuron activity and induction of depressive-like behavior by high-frequency stimulation of the subthalamic nucleus. Proc Natl Acad Sci U S A 104, 17087-17092.

24. Temel, Y., Kessels, A., Tan, S., Topdag, A., Boon, P., and Visser-Vandewalle, V., 2006. Behavioural changes after bilateral subthalamic stimulation in advanced Parkinson disease: a systematic review. Parkinsonism Relat Disord 12, 265-272.

25. Witt, K., Daniels, C., Reiff, J., Krack, P., Volkmann, J., Pinsker, M. O., Krause, M., Tronnier, V., Kloss, M., Schnitzler, A., Wojtecki, L., Botzel, K., Danek, A., Hilker, R., Sturm, V., Kupsch, A., Karner, E., and Deuschl, G., 2008. Neuropsychological and psychiatric changes after deep brain stimulation for Parkinson's disease: a randomised, multicentre study. Lancet Neurol 7, 605-614.

26. Zangaglia, R., Pasotti, C., Mancini, F., Servello, D., Sinforiani, E., and Pacchetti, C., 2012. Deep brain stimulation and cognition in Parkinson's disease: an eight-year follow-up study. Movement disorders : official journal of the Movement Disorder Society 27, 1192-1194.

27. Zibetti, M., Merola, A., Rizzi, L., Ricchi, V., Angrisano, S., Azzaro, C., Artusi, C. A., Arduino, N., Marchisio, A., Lanotte, M., Rizzone, M., and Lopiano, L., 2011. Beyond nine years of continuous subthalamic nucleus deep brain stimulation in Parkinson's disease. Movement disorders : official journal of the Movement Disorder Society 26, 2327-2334. 
SUMMARY 
Parkinson disease (PD) is a progressive neurodegenerative disorder. In Europe 108 out of 100.000 people suffer from PD. The key motor symptoms are tremor, rigidity, bradykinesia and postural instability, also non-motor symptoms such as cognitive impairments and mood changes appear. PD is neuropathologically recognized by loss of dopamine (DA) cells and the presence of Lewy bodies. In the early stages of the disease motor symptoms can be adequately improved by levodopa treatment. Unfortunately, the beneficial effects wear off progressively and 'on-off' fluctuations and levodopa induced dyskinesias appear.

The subthalamic nucleus (STN) plays an important role in the pathophysiology of PD. STN neurons in PD patients and in animal models of PD show a bursty firing pattern. Parkinsonian motor symptoms can be alleviated by both STN inactivation and ablation and high frequency electrical stimulation. Since the introduction of deep brain stimulation of the subthalamic nucleus (STN DBS) in 1993, this therapy has proven to be effective on the short and long term. In advanced stages of PD gait problems occur, which leads to frequent falls. Current STN DBS treatment has no effect on these axial symptoms.

The STN in contrast to al other basal ganglia nuclei is excitatory. STN neurons receive major input from the cortex and globus pallidus externus (GPe) and are under influence of the monoaminergic systems. Within the cortico-basal ganglia thalamocortical circuit, the STN plays an important role in not only motor, but also in cognitive and limbic processes. Classically the STN is divided into three subregions: a motor, limbic and associative part. During the last decade the monosynaptic cortico-subthalamic pathway, also known as the 'hyperdirect' pathway regained new interest. Currently, two major theories for cortico-basal ganglia information processing for motor, associative and limbic information exist. The first supports a parallel flow of information through the cortico-basal ganglia circuits and the second supports convergence of these pathways, while others consider parallel pathways with interaction between them. The question whether the monosynaptic cortico-subthalamic pathways are organized in a strictly parallel or convergent manner is still not clearly answered.

Unfortunately, STN DBS has not only positive effects on the motor symptoms, but also affects mood and cognition in a substantial number of patients. The challenge is to obtain good therapeutic effects and to prevent the occurrence of undesired psychiatric side effects. The undesired psychiatric side effects are thought to be caused by stimulation of the non-motor parts of the STN and the influence on the serotonergic system. Another challenge is to treat the axial motor symptoms. In this thesis we test the feasibility of reducing psychiatric side effects of STN DBS. Therefore, we conducted a set of experimental and clinical studies to investigate the mechanisms of how STN DBS can be improved. 
Only few studies reported a long term follow-up with data up to ten years after surgery. Their results indicated a persistent effect of stimulation on the core motor symptoms but an increase in disability and cognitive impairment due to disease progression. We started our STN DBS program for PD patients in 1999. In chapter 1, we report on the motor and cognitive outcome up to 10 years after surgery. Both motor and non-motor performances were extensively evaluated pre-operatively and at 3 months, 1 year, 5 years and 10 years after surgery. We operated on twenty-seven patients more than 10 years ago. Twelve patients completed the 10 year follow-up. At baseline, patients who completed the total follow-up were younger (54 years of age) than those who did not (60 years). In the early post-operative phase a strong improvement of the motor scores on the UPDRS III and IV and levodopa equivalent dose (LED) reduction was found. However, after five years a gradual worsening of the motor performance was observed. At ten years follow-up the UPDRS III scores were slightly worse than the pre-operative medication ON condition, but still better than the pre-operative medication OFF condition. The worsening in motor performance was almost exclusively due to a deterioration of bradykinesia and axial symptoms. Depression scores did not change over time. However, one third of the patients showed impulsive behavior after surgery. Especially younger patients seemed to be at risk for impulsive behavior. A decrease in performance on executive tests, on the other hand, seems to be associated with higher age and an advanced stage of the disease. We concluded from this study that optimization of the targeting of the motor part of the STN and preventing stimulation of the limbic and associative parts of the STN might help to reduce stimulation induced behavioral side effects. There is also a need for new therapies to specifically treat the axial symptoms which determine the quality of life in these patients 10 years after surgery. Axial symptoms do not respond well to DA replacement therapies. Therefore, these symptoms are more likely related to the non-dopaminergic, monoaminergic systems.

As stated above, despite improving motor disability, in some patients STN DBS induces mood disorders such as depression and increased impulsivity. In addition, evidence suggests that the risk of suicide increases significantly. Depression, impulsivity and suicide are associated with a dysfunctional serotonin (5-HT) system. The neural circuitry underpinning the effect of high frequency stimulation (HFS) of the STN on 5-HT neurons remains unknown. In chapter 2 we investigated in the dopamine-denervated rat the effect of bilateral HFS of the STN on markers of neuronal activity in the DRN as well as DRN input regions. HFS of the STN elicited an increase in immobility time compared to non-stimulated controls, indicating increased behavioral despair. In the social interaction task, HFS of the STN evoked significantly more sniffing behavior. Both behavioral effects are consistent with decreased 5-HT transmission. HFS of the STN at the same stimulation parameters, increased c-Fos immunoreactivity in the DRN, and decreased cytochrome $\mathrm{C}$ oxidase activity in this region. The increase in c-Fos immunoreac- 
tivity occurred in DRN neurons immunopositive for the GABA marker parvalbumin. HFS of the STN also increased the number of c-Fos immunoreactive cells in the lateral habenula nucleus (LHb), medial prefrontal cortex (mPFC). These data are consistent with the view that HFS of the STN activates DRN GABA neurons to inhibit 5-HT neuronal activity and trigger mood changes, and that DRN afferents from the LHb and MPFC may be involved.

Besides the influence of the 5-HT systems, the neuropsychiatric side-effects induced by STN DBS are thought to be caused by direct stimulation of the associative and limbic pathways that run through the STN. In the primate, three functionally segregated parts are clearly described within the STN: a dorsolateral motor part, a medial limbic part and a ventrolateral associative part. In the rodent however, these subdivisions are not well defined. In this review in chapter 3 we describe all anterograde corticosubthalamic tracer studies to map the rodent STN. As a result, a crude functional subdivision in the rodent STN could be made. Nevertheless, it seems unlikely that the subdivisions of the rat STN are entirely segregated from each other. Dendrites can extend across almost the entire STN. Despite noteworthy differences between the rat STN and the (human) primate STN the internal organization and its place in the basal ganglia is highly comparable. We concluded that a partial anatomical subdivision system is present in the rodent STN, although it is not as clear cut as in the primate. Neurons in the medial STN mainly get their afferent input from the limbic and associative cortical areas and those in the lateral two thirds receive their input from the motor areas.

The STN's involvement in limbic functions can be explained by its connections to cortical and subcortical limbic regions via the cortico-basal ganglia-thalamocortical circuits. In chapter 4 we addressed the research question whether the medial part of the STN is involved in the mood-related effects of stimulation of the NAc, VMPFC, and Cg. We found that HFS of the NAc and VmPFC, but not Cg showed an antidepressant-like response. In addition, HFS of the VmPFC, but not NAc and $\mathrm{Cg}$, enhanced c-Fos-ir specifically in the medial part of the STN. Although the medial STN is generally regarded as an integrator and processes limbic and associative information, our results suggest that the medial STN has specific functional roles for depressive-like behaviours that depend largely on which limbic projection pathway was stimulated.

In this chapter we used an extracellular electrophysiological approach, to analyze the organization of the STN motor and non-motor subdivisions in the normal and DA depleted rodent brain. Our results showed that most STN neurons were responsive to cortical stimulation. From the responsive neurons, the majority responded to stimulation of two or more cortical areas. Interestingly, responses to different cortical areas were mainly seen with a long latency. These long latency responses are related to the 'indirect' cortico-striato-pallido-subthalamic pathway. Frequently a combination of short and long latency responses were observed, which have been earlier characterized 
as a triphasic response. A large number of STN neurons selectively responded to either motor or non-motor cortical regions with a crude somatotopy. Indeed, the responsive neurons to motor cortex stimulation were mainly located in the lateral portion of the STN, whereas neurons responding to stimulation of limbic/associative cortical areas were mainly seen in the medial parts of the STN. On the contrary, it should be noticed that responsive neurons to the stimulation of motor and non-motor cortices were not limited to these areas and functional territories largely overlapped. In the 6-OHDA treated rats no differences were seen in the functional anatomical localization of STN neurons compared to the sham group. Our findings are in agreement with a basal ganglia theory which presumes parallel cortico-basal ganglia pathways with interaction between them. For STN DBS in PD patients this could suggest that avoidance of behavioral side effects by selective stimulation of the motor part solely is hard to achieve, since a pure motor part may not exist.

The aim of the study presented in chapter 6 and 7 was to identify the STN motor area in PD patients by using motor cortex stimulation (MCS) in order to reduce STN DBS induced behavioral side effects. We tested the feasibility of identifying the STN motor part by MCS and evaluated the safety of this novel approach. A strip of four electrodes was placed in the subdural space through a burr hole posterolateral to the hand area during the stereotactic procedure in 5 patients. After baseline recordings, cortical evoked neuronal activity was measured. We observed significant excitations and inhibitions as a response to MCS. Responses varied between different depths and between different locations in the anterior-posterior and medio-lateral plane within the STN. Studies in rats and primates showed typical triphasic responses, consisting of an initial excitation, a subsequent inhibition and a second excitation. It is likely that the difference in methodology is responsible for the lack of clear tri-phasic responses in human studies. We also showed that evoked LFPs follow a specific pattern in the dorsal STN. The spatial resolution of the responses measured in the LFP to MCS is not high enough to identify the STN motor region. We believed that the burden of the affective and cognitive side effects outweighed the risks of the MCS procedure. An important limitation of subdural MCS in our study is the occurrence of partial seizures. The risk of a seizure is related to the applied current and current density. To prevent seizures the current density should be lowered, so that motor cortex stimulation evoked responses can be safely used during deep brain stimulation surgery.

Falls, freezing, festination, and postural instability become more pronounced in late-stage PD. This is also reflected by the motor deficits seen in the long-term followup study after STN DBS surgery as seen in chapter 1 . Here, we investigated the constancy and reproducibility of gait parameters in the CatWalk in an animal model of PD. We demonstrated in chapter 8 that the gait pattern of rats is highly reproducible in the CatWalk. 6-OHDA lesioned rats display impairments in gait, and levodopa can counter- 
act these to a certain amount. In view of the fact that gait disturbances are a major cause of morbidity and even mortality in PD, further preclinical investigations to find underlying mechanisms for gait problems in PD is needed.

Although altered STN activity is observed in PD patients in the advanced stages of the disease as well as in animal models with severe DA lesions, it is still elusive at which stage of the disease changes in STN neuronal activity occur. In the current study, we addressed this by evaluating electrophysiological characteristics of STN neurons and the motor behaviour in an animal model mimicked early stages of PD. Aside from a slower motor response in the reaction time task, animals with a mild lesion displayed no gross locomotor abnormalities. However, the firing pattern of their STN neurons shifted from a more regular firing type to a burst mode. Animals with a substantial DA lesion, showing the same slowing in the motor response, had a similar shift in the firing pattern. Thus, the electrophysiological properties of the STN already shifted to a bursty phenotype due to a mild DA depletion before severe motor symptoms were present. A provocative hypothesis is that hyperactivity of the STN is compensatory mechanism to drive the remaining SNc neurons to enhance the dopamine release through the nigrostriatal pathway.

In conclusion, our findings underline the fact that the STN possesses a key position in the cortico-basal-ganglia-thalamo-cortical network. The STN is strongly involved in motor, cognitive and limbic processes. A strict tripartite of STN subdivision can however not be made. At the single neuron level, cognitive, limbic and motor processes are being integrated. From a clinical point of view, STN DBS is an effective therapy to treat PD symptoms, though further reduction in psychiatric side-effects remains a challenge. Improvement of targeting by using enhanced imaging techniques, improved electrophysiological recordings, and better stimulation electrodes might allow us to reduce undesired neuropsychiatric side-effects and enhance the therapeutic benefit. To improve axial symptoms in PD patients the focus for DBS treatment should be on the brainstem and cerebellar nuclei, and the influence of the non-dopaminergic monoamines on gait needs further assessment. 
SAMENVATTING 
De ziekte van Parkinson is een progressieve neurodegeneratieve ziekte. Patienten met deze ziekte hebben met name last van beven, stijfheid, traagheid van bewegen en verminderde balans. Daarnaast hebben deze patienten ook last van achteruitgang van geheugen en stemmingsproblemen. Deze symptomen ontstaan door een tekort van een stof in de hersenen, genaamd dopamine. In het begin van de ziekte kunnen de bewegingsproblemen goed behandeld worden door het tekort van dopamine aan te vullen met medicatie (levodopa). Helaas wordt het effect van de medicamenteuze behandeling langzaam minder en krijgen patienten last van zogenaamde "on-off" fluctuaties en overbeweeglijkheid.

Binnen de ziekte van Parkinson speelt de nucleus subthalamicus (STN) een belangrijke rol. De bewegingssymptomen kunnen behandeld worden door een kleine laesie in deze kern te maken, of door deze te inactiveren door hoog frequentie electrische pulsen toe te dienen. Deze techniek heet diepe hersenstimulatie (DBS) en wordt sinds 1993 wereldwijd toegepast. Bij patienten met een verder gevorderd stadium van de ziekte treden problemen op met lopen en de balans, waardoor patienten vaak vallen. DBS van de STN heeft geen effect op deze symptomen.

De STN is een interessante kern binnen de hersencircuits (basale kernen) die betrokken zijn bij de ziekte van Parkinson. Deze kern speelt een rol in het controleren van de motoriek, geheugenprocessen en emotionele processen. Deze structuur wordt in de literatuur onderverdeeld in drie verschillende functionele gebieden: een motorisch, een gebied voor geheugenprocessen en tot slot een gebied voor emotionele processen. Er bestaan binnen de huidige onderzoekers twee tegenstrijdige hypothesen, waarbij de eerst stelt dat informatie vanuit de opervlakkige hersenen via parallele banen via de basale kernen verloopt en de tweede stelt dat deze informatie samen gebracht wordt. Daarnaast zijn er ook nog onderzoekers die parallele circuits veronderstellen met interactie hiertussen. De vraag blijft tot op heden echter onbeantwoord in welke mate informatie vanuit de oppervlakkige hersenen van motorische, geheugen en emotionele processen in de STN samenkomt.

Wetende dat de STN ook een rol speelt bij verschillende niet-motorische processen, is het niet verrassend dat STN DBS niet enkel positieve effecten heeft op het bewegen, maar ook soms veranderingen geeft in het gedrag en de stemming van patiënten. Het is dan ook de uitdaging voor artsen en onderzoekers om een zo goed mogelijk effect van de stimulatie op het bewegen te verkrijgen, zonder dat er ongewenste neveneffecten optreden. Deze neuropsychiatrische neveneffecten komen waarschijnlijk door stimulatie van de niet-motorische gebieden van de STN, waarbij beinvloeding plaatsvindt van gebieden (dorsale raphe nucleus, DRN) waarvan bekend is dat zij een stof (serotonine) produceren die een belangrijke rol speelt bij depressie. Een ander probleem welk verder onderzoek verdient zijn de, voor behandeling resistente, balansproblemen. In deze thesis hebben wij onderzocht of het mogelijk is om de psychiatrische 
bijwerkingen te voorkomen die soms optreden ten gevolge van STN DBS. Onze hypothese was dat dit mogelijk was door specifiek het motorische gebied van de STN te identificeren en te stimuleren. Hiertoe zijn een aantal studies verricht.

Allereerst hebben we in onze eigen patiëntenpopulatie onderzocht wat de effecten zijn van STN DBS. In onze patiëntengroep vonden we dat het bewegen na behandeling met DBS duidelijk verbeterde. Echter na 5 jaar werd dit effect langzaam minder. 10 jaar na de operatie was met name de balans verstoord en de traagheid van bewegen toegenomen. Gemiddeld genomen was er geen toename van depressieve symptomen, wel werd bij een derde van de patiënten een toename van impulsiviteit gezien. Problemen met de geheugenfuncties was met name aanwezig bij de oudere patiënten. Vanuit deze studie hebben we geconcludeerd dat er verbetering wenselijk was voor de plaatsing van electroden in de STN om bijwerkingen te voorkomen. Ook is er een grote behoefte aan nieuwe behandelmogelijkheden om de balansproblemen te beteugelen.

Om uit te zoeken waardoor er ten gevolge van STN DBS stemmingsproblemen ontstaan hebben wij bij proefdieren naar de functionele verbindingen tussen de STN en de DRN gekeken. Een ratmodel voor de ziekte van Parkinson is gebruikt en deze ratten zijn geimplanteerd met een electrode in de STN, die vervolgens gestimuleerd is. Hierbij vonden we veranderingen in het gedrag van de ratten die gerelateerd zijn aan het serotonine systeem. Dit veranderde gedrag kon verklaard worden door een activatie van neuronen in de DRN die een remmende werking hebben op de serotonine producerende neuronen. De beinvloeding van deze neuronen verloopt indirect.

Naast de beinvloeding van het serotonine systeem zijn de gedragsverandering ook deels een direct effect door beinvloeding van de niet-motorische gebieden in de STN door de stimulatie. In de primaat zijn deze gebieden duidelijk gedefinieerd. Hoe dit in ratten zit is echter niet geheel duidelijk. Na het bestuderen van de literatuur konden we concluderen dan er een minder strikte onderverdeling van functionele subgebieden gemaakt kan worden in de STN bij ratten. De functionele subgebieden zijn waarschijnlijk deels overlappend. Vervolgens hebben we een experimentele studie verricht in ratten waarbij we keken naar de beïnvloeding van depressief gedrag door DBS van verschillende hersenstructuren in het brein. Deze studie liet zien dat als stimulatie van een specifieke hersenstructuur het depressieve gedrag beïnvloedde, er ook een activatie te zien was in het mediale deel van de STN. Deze studie onderstreept de betrokkenheid van de STN bij depressief gedrag. Vervolgens hebben we verschillende electrische pulsen toegediend in verschillende gebieden van de oppervlakkige hersenen in ratten. De electrische signalen van de neuronen in de STN veranderen hierdoor. Middels deze techniek konden we aantonen dat er, zoals we ook al in de literatuur gezien hadden, een grove onderverdeling is van de functionele subgebieden in de STN van de rat, maar dat ook een deel van de cellen informatie ontvangt vanuit de oppervlakkige hersenen die zowel betrokken zijn bij het bewegen, maar ook bij het gedrag. Deze bevinding is nieuw en 
impliceert dat een puur, selectieve stimulatie van enkel de motorische banen in de STN mogelijk niet haalbaar is.

Om de optredende bijwerkingen van gedragsveranderingen te voorkomen hebben we een haalbaarheidsstudie uitgevoerd. Hiertoe hebben we tijdens de operatie van de Parkinson patiënten een extra handeling uitgevoerd. Terwijl we naar de electrische signalen van de neuronen in de STN keken, gaven we ook electrische pulsen op de oppervlakkige hersenen die bewegingen aansturen. Deze resulteerden in signalen die meetbaar waren in de STN, echter waren deze onvoldoende specifiek om op basis hiervan een verbetering van de electrode-plaatsing te bereiken. Helaas traden er ook epileptische aanvallen op ten gevolge van de stimulatie. Deze bijwerkingen waren onverwacht en ongewenst. Om deze te voorkomen moet bij toekomstig onderzoek de hoeveelheid stroom verlaagd worden.

Naast de gedragsbijwerkingen stuitten we op nog een ander probleem bij patiënten die een gevorderd stadium van de ziekte bereikt hebben, namelijk de balans- en loopproblemen. Om hiervoor een behandeling te vinden, hebben wij in een ratmodel van de ziekte van Parkinson getest of we de gang betrouwbaar kunnen meten in dit diermodel. De CatWalk bleek een betrouwbaar meetinstrument om multiple aspecten van het gangpatroon te analyseren. Een deel van de gangproblemen reageerden ook op levodopa behandeling, maar niet alle parameters verbeterden. Tot slot hebben we ook gekeken in een ratmodel van de ziekte van Parkinson wanneer de veranderingen van het vuurpatroon van de STN neuronen optreden. We vonden dat bij ratten met minimale bewegingsproblemen al de 'burst' activiteit toegenomen was. Mogelijkerwijs is dit een compensatiemechanisme om de neuronen die dopamine produceren aan te sporen.

Als we de bevindingen samenvatten van de onderzoeken die we gedaan hebben, komen we tot de volgende conclusie: de STN heeft een centrale positie in de basale kernen. De STN is betrokken bij beweging, maar ook bij geheugen en emotionele processen, zoals bijvoorbeeld depressie. Zoals eerder verondersteld zijn de functionele gebieden van de STN niet geheel gescheiden. Als we inzoomen, dan zien we dat individuele neuronen in de STN zowel informatie van motoriek als van het gedrag verwerken. Terugkomend op onze hypothese betekenen deze bevindingen voor de klinische praktijk dat het een grote uitdaging blijft om de gedragsbijwerkingen van STN DBS te verminderen. Daarnaast is er grote behoefte voor de behandeling van de gang en balansproblematiek waarvoor tot op heden een effectieve behandeling ontbreekt. 
Valorisation 
Parkinson disease is a progressive neurodegenerative disorder. In Europe 108 out of 100.000 people suffer from Parkinson disease. The key motor symptoms are tremor, rigidity, bradykinesia and postural instability, also non-motor symptoms such as cognitive impairments and mood changes appear. Parkinson disease is neuropathologically recognized by loss of dopamine cells in a specific part of the brain and the presence of Lewy bodies (a misfolded proteïn). In the early stages of the disease motor symptoms can be adequately improved by oral dopamine drug replacement therapy. Unfortunately, the beneficial effects wear off progressively and 'on-off' fluctuations and L-dopa induced dyskinesias appear. In this stage of disease deep brain stimulation is the first choice therapy. Since the introduction of deep brain stimulation of the subthalamic nucleus in 1993, this therapy has proven to be effective on the short and long term. However, in a substantial number of patients neuropsychiatric side effects are seen. In this thesis we investigated the pathophysiological mechanisms of these side-effects and tried to overcome these by pre-clinical experiments in an animal model of Parkinson disease and by an electrophysiological approach in Parkinson patients during deep brain stimulation surgery.

In the first chapter of this thesis we show the short and long term outcome of subthalamic nucleus deep brain stimulation in our cohort of patients. We learned that some neuropsychiatric symptoms are present in some patients. We have seen an increased impulsivity in younger patients, whereas general cognitive problems were seen in older patients. As reported in literature we also have seen deterioration in gait after 10 years. This chapter shows that the issues raised in the international literature are also present in the Dutch population and therefore in need for further studies to overcome these problems.

In chapter 2 we investigated the behavioral side-effects in a rodent. We used a widely used model for Parkinson disease, namely the 6-OHDA model. We found behavioral deficits which are related to the serotonin system, which is involved in depression. Next we tested the connection between the subthalamic nucleus and the dorsal raphe nucleus, a brainstem region in which serotonin is produced. We found changes using immuno-histochemical markers, which underline the fact that deep brain stimulation of the subthalamic nucleus affects the serotonin system. This finding led to further scientific investigations in animal models as well as in Parkinson patients and might be a first step in overcoming depressive symptoms induced by subthalamic nucleus stimulation.

In chapter 3 a review of the literature was performed to investigate the cortical connections with the subthalamic nucleus. The findings of this literature review were necessary for the experiments in chapter 4 and 5. 
In chapter 4 we tested deep brain stimulation in certain brain areas in animals to treat depression. The mechanism behind the efficacy of stimulation of these areas is still not fully understood. Since the STN is also connected to these areas we investigated which part and to what extend the STN is involved. The findings of this experiment help the scientific community to understand the mechanisms behind the efficacy of deep brain stimulation in depression and might in the end lead to novel therapeutic options for depressive patients.

In chapter 5 we tested if the subthalamic nucleus has three independent subdomains with different functionality. We tested this using an electrophysiological approach in an animal model. We learned that there is a partition seen, but overlap is present. This finding has clinical implications for deep brain stimulation of the subthalamic nucleus in Parkinson patients. Pure selective stimulation to reduce motor symptoms without inducing undesired behavioral side effects might thus not be possible. This has direct consequences for these patients.

In chapter 6 and 7 we aimed to overcome undesired side-effects of deep brain stimulation of the subthalamic nucleus in Parkinson patients by applying stimulation of the superficial brain region which corresponds with motor function. This technique was promising and if the responses acquired would have been clearer, it would have been possible to further improve the motor symptoms without inducing the undesired behavioral side-effects. This would have reduced medical costs significantly.

In chapter 8 we assessed a novel tool to assess gait in an animal model of Parkinson disease. Gait as shown in chapter 1 is a major problem in patients with advanced stages of Parkinson disease. Thus far no therapies are available to treat this symptom. Falls lead to hospital admissions and hip-fractures. This study was necessary to be able to further investigate gait problems in animal models to be able to develop novel therapies for the gait disturbances. If a treatment can be developed this leads to lesser burden for the patients and caregivers, as well as a reduction in medical costs.

In chapter 9 we performed a basic scientific experiment which helps us to understand the pathophysiology in Parkinson disease. The results of this experiment contributed to the basic knowledge of the scientific community.

In summary, the experiments performed in this thesis consisted of clinical experiments with patients, experiments with animal models and a review of the literature. The scientific approaches used were well reasoned. The approaches chosen depended on the research question which needed to be answered. Although we are aware that the trans- 


\section{VALORISATION}

lation of animal data to humans is often difficult, animal research is indispensable to establish advances in neuroscience. In my opinion, not only clinical studies, but also preclinical experiments using animal models are thus necessary to sustain the high quality of our medical care and basic scientific knowledge. I therefore encourage the European and Dutch Government, as well as private funding programs to keep funding basic scientific research in which animal research is involved. 
Dankwoord 
Uiteraard zijn er velen die mij geholpen hebben bij het tot stand komen van mijn proefschrift. Dit boekje had ik nooit zonder de hulp en steun vele mensen om mij heen voor elkaar gekregen. Enkele personen wil ik in het bijzonder noemen, te beginnen met mijn promotoren.

Prof. Dr. V. Visser-Vandewalle

Beste Veerle, jij was diegene die mij vroeg of ik aan dit promotie project wilde beginnen. Wat ben ik dankbaar met het vertrouwen dat jij in me had. Onze gesprekken heb ik altijd als zeer fijn ervaren. Bedankt hiervoor! Wat ben ik trots dat jij nu afdelingshoofd bent van de afdeling functionele neurochirurgie in Keulen.

Prof. Dr. Y. Temel

Beste Yasin, het is ongelofelijk wat jij hebt betekent voor mij de afgelopen jaren. Door je klinische werkzaamheden kon je niet altijd aanwezig zijn in het lab, maar dit compenseerde je volledig met je passie voor het onderzoek. Je weet mij steeds weer opnieuw te enthousiasmeren voor het onderzoek. Dank voor al je goede adviezen de afgelopen jaren. Ook wil ik graag Halime en je dochters bedanken voor de vele avonduren dat ik bij jullie te gast mocht zijn. Ik hoop dat ik nog vaak mag langskomen.

\section{Prof. A. Benazzouz}

Cher Hamid, je vous remercie de m'avoir fait venir à Bordeaux pour apprendre la technique électrophysiologique chez les rats parkinsoniens. Je n'oublierai jamais la chaleur avec laquelle j'ai toujours été accueilli. Votre connaissance des ganglions de la base et de la maladie de Parkinson est extraordinaire. J'ai beaucoup apprécié votre patience avec l'écriture de l'article. C'est bien aimable de votre part.

Naast mijn promotoren zijn er vele anderen die hebben bijgedragen aan de tot stand koming van dit proefschrift. Allereerst wil ik Daphne bedanken voor de mooie samenwerking tussen Twente en Maastricht. De fysieke afstand heeft ons gemeenschappelijke doel nooit in de weg gestaan. Vanaf het begin van ons promotie traject hebben we elkaars werk gecomplementeerd. Ik vind het prachtig dat je hebt laten zien dat promoveren en moeder worden samen gaat. Beste Cisca, bedankt voor je begeleiding en altijd constructieve commentaar op onze data en papers. Ik kijk uit naar de toekomst om onze samenwerking een mooi vervolg te geven. Beste Annelien, voor de DBS patiënten ben jij een onmisbare schakel in het gehele operatie traject. De vele mailwisselingen over de data en papers hebben zich uitbetaald. Bedankt voor je altijd constructieve input. Beste Vivianne, wat had je het toch altijd druk, toch mocht ik altijd binnenlopen en wist je altijd tijd te maken voor mijn onderzoek. Bedankt voor je hulp bij de stimulatie en recordings. Dear Jenny, I wish that writing a paper with someone would always go 
so smoothly as with you. Thank you for the nice collaboration we had. Beste Govert, het was altijd fijn om even bij jou stoom te af te blazen. Voor mij zijn jouw kennis en kunde van onmiskenbare waarde voor onze onderzoeksgroep. Beste Nicole, bedankt voor al je hulp bij het verzamelen van de patiënten data. Zonder jouw hulp hadden we nooit alle klinische data bij elkaar gekregen. Alle dames van de adminstratie van de afdelingen neurochirurgie en MHeNS verdienen een pluim voor hun werk achter de schermen. Marie-José en Lisa Pizzuto, jullie stonden altijd klaar als er iets geregeld moest worden. Geert-Jan, Inge, Jessie, Mark, Ruud en Yara bedankt voor de vormgeving, vertaling en correcties van het proefschrift.

De patiënten die deel hebben genomen aan mijn studie wil ik in het bijzonder bedanken voor deelname aan een onderzoek welk niet zonder risico's was. Ook wil ik even stil staan bij de proefdieren die gebruikt zijn voor de preklinische onderzoeken.

\section{Mijn collega's in het lab}

Wat hadden we een toch een mooi onderzoeks team. Een groepje gekke, jonge, gedreven onderzoekers die het beste in elkaar naar boven haalt. ledereen hielp elkaar en stuurde aan waar nodig. Naast de tijd samen op het lab hebben we ook van vele, onvergetelijke feestjes en congres reisjes mogen genieten.

Beste Sonny, ik ben er bijzonder trots op dat ik eerst als student en later als collega samen met je heb mogen werken. Jij hebt het vlammetje voor de wetenschap bij me aangewakkerd. Wat was het fijn dat we zowel op de werkvloer goed samen konden werken, maar ook altijd gezellig samen een biertje konden drinken. Beste Rinske, je was altijd al de "mommy" van de groep en nu ben je echt moeder geworden. Wat werkte je altijd netjes, bedankt voor alle zo mooi uitgeschreven protocollen en je helpende hand bij mijn experimenten. Dear Anthony, your working spirit and motivation has always been an example to me. We all miss your charisma in the lab. Dear Tibo, you were wrapping up your thesis when I started. Thanks for teaching me all necessary lab skills. Dear Ali, I really miss the coffee brakes we always had together. I admire you for your dedication and technical skills. If I need help, I can always depend on you. You are great colleague and friend.

Uiteraard wil ik ook alle studenten die mij geholpen hebben enorm bedanken voor jullie hulp. Soms werd ik helemaal gek van jullie, omdat ik niet steeds maar niet verder kon met mijn eigen werk, maar uiteindelijk was dit alles nooit gelukt zonder jullie inzet. Ook dank aan alle andere collega's en vrienden in het lab voor de collegiale, maar vooral ook voor de gezellige tijd die we samen hebben gehad. De labdagjes uit en het Society for Neuroscience congres in San Diego zal ik niet gauw vergeten.

Daarnaast wil ik ook de technicians en de medewerkers van de centrale proefdiervoorziening bedanken voor hun hulp en ondersteuning bij het uitvoeren van de dierexperimenten. 
Daarnaast ook mijn dank aan alle huidige collega's in het lab voor de hulp nadat ik het lab reeds verlaten had. Zonder jullie waren de experimenten van Filipa en Emily nooit tot een goed eind gekomen.

\section{Mes colleagues en Bordeaux}

Je veux dire un gros merci à tous mes collègues et mes amis à Bordeaux. Merci à tous et à toutes pour expliquer tout à nouveau en anglais. Grâce à vous, j'ai facilement pu m'adapter à Bordeaux. Claire merci pour votre aide avec mes expérimentations, sans votre aide je n'aurais jamais réussi à expérimenter sur les animaux. Je pouvais toujours compter sur vous et sur votre voiture fantastique. Melanie, merci pour expliquer l'équipement électrophysiologique en partageant l'appareil. Phillipe, ton café était toujours fort et délicieux. Personne ne peut expliquer le hplc mieux que vous.

\section{Mijn collega's bij de neurologie}

$\mathrm{Na}$ mijn periode als onderzoeker kreeg ik het vertrouwen van mijn opleiders van de vakgroep neurologie om te starten als AIOS neurologie. Ik ben de stafleden hier erg dankbaar voor. Het was even wennen om geen rattendokter meer te zijn. Mijn collega arts-assistenten wil ik bedanken dat ze mij, met name in het eerste jaar van mijn opleiding, zoveel geholpen en gesteund hebben om weer een 'echte' dokter te zijn. Ik voelde me meteen thuis in de assistentengroep. Dit komt met name door mijn lieve jaargenoten. Martijn, samen met jou als kamergenoot zijn alle cursussen een feest. Jouw droge humor blijft fantastisch, al moeten we wel nog wat werken aan je muziek keuzes. Gelukkig loopt het onderzoek bij jou nu ook goed. Bedankt dat je nu als paranimf naast mij wilt staan, over een paar jaar mag jij in het midden.

\section{Mijn vrienden en oud huisgenoten}

Heerlijk was het, om naast het harde werken ook weer eens het verstand op nul te zetten. Zonder de gezellige momenten in de bar, op de fiets of op vakantie had ik het nooit vol gehouden. Wat is het toch mooi om een stel vrienden te hebben die vanuit Nederland een weekendje op en neer rijden naar Bordeaux.

Tom, jou wil ik van al mijn vrienden toch nog speciaal even noemen. Wat hebben we samen in Heugem een mooie studententijd gehad. De studeer, game en stapavonden wisselenden elkaar in rap tempo af. Ik ben blij dat ik jou als paranimf aan mijn zijde mag hebben.

\section{Mijn familie}

Altijd waren jullie oprecht geintereseerd in mijn onderzoek. Door mijn onderzoek, mede door mijn periodes in Bordeaux, had ik vaak te weinig tijd voor jullie. Oma, Rob en Loes, 
mijn zus Ellen, mijn schoonouders en Inge, en uiteraard mijn altijd vrolijke nichtje Sophie en neefje Thomas, een betere familie kan men zich niet wensen.

Lieve Ellen, zonder jou was dit proefschrift nooit afgekomen. Al mijn frustraties kon ik steeds weer bij jou kwijt. Jij zorgde ervoor dat ik mijn zinnen weer kon verzetten als alles tegen zat. In mijn drukste onderzoeksperiodes zorgde jij ervoor dat ik niet vergat te eten. Nooit deed je moeilijk als ik weer eens naar het buitenland toe ging of een avond geen tijd voor je had. Bedankt voor je liefde en steun!

Papa en mama, jullie zijn voor mij altijd heel belangrijk geweest bij al de keuzes die ik gemaakt heb in mijn leven. Ik wil jullie bedanken voor het fijne thuis waarvan ik altijd nog mag genieten. Voor raad en hulp kan ik altijd bij jullie terecht, zonder dat daar ooit iets tegenover hoefde te staan. Ik wil dit boekje dan ook aan jullie opdragen. Bedankt voor alle talenten die ik van jullie heb gekregen! 



\section{Biography}

Mark (Marcus Leo Franciscus) Janssen was born on 24 September 1984 in Heerlen, The Netherlands. He is the second child and son of Hendrik Hubert Janssen (1956), born in Kerkrade, married with Maria Theresia Leonarda Louise Huppertz (1960), born in Heerlen. In 1988 he went to the primary school 'De Horizon'. From 1996-2002 he followed his education at the Lyceum of the Sintermeerten college, Heerlen. He opted for the study direction Science and Health. In 2002, 17 years old, he entered medical school at Maastricht University and moved to Maastricht. Throughout his medical study he worked as a research assistant at the Maastro Clinic (radiotherapy institute). During his medical study, he became fascinated by the brain and more specifically by deep brain stimulation. Therefore, during his research internship he investigated the role of the serotonergic system on the side effects of deep brain stimulation of the subthalamic nucleus under supervision of Dr. S. Tan, Prof. Y. Temel and Prof. H. Steinbusch. During his last clinical rotation he was trained at the Department of Neurosurgery at the Maastricht University Medical Center under the supervision of Prof. Visser-Vandewalle and at the Department of Functional Neurosurgery at the Montreal Neurological Institute in Montreal, Canada under the supervision of Prof. A. Olivier. In 2008, he finished his medical study and spoke the Hippocratic Oath. Immediately after his studies he started his doctoral research at the School for Mental Health and Neuroscience at Maastricht University supervised by Prof. V. Visser-Vandewalle and Prof. Y. Temel. The aim was to identify the motor area of the subthalamic nucleus. As a PhD student, he spent a year in the lab of Prof. A. Benazzouz in the Institut des Maladies Neurodégénératives at the University of Bordeaux to perform in vivo electrophysiology in the dopamine depleted rat. Currently, he works as a resident at the Department of Neurology at the Maastricht University Medical Center. 



\section{List of publications}

Journal articles

- Janssen ML, Temel Y, Duits AA. Diepe hersenstimulatie van de nucleus subthalamicus voor de ziekte van Parkinson: de neuropsychiatrische gevolgen. Tijdschrift voor neuropsychiatrie en gedragsneurologie. Accepted

- $\quad$ Smit JV, Janssen ML, Schulze, H, Jahanshahi A, Van Overbeeke JJ, Temel Y, Stokroos RJ. Deep brain stimulation in tinnitus: current and future perspectives. Brain Res. 2015. In press.

- $\quad$ Lim LW, Janssen ML, Kocabicak E, Temel Y. The antidepressant effects of ventromedial prefrontal cortex stimulation is associated with neural activation in the medial part of the subthalamic nucleus. Behav Brain Res. 2015 Feb; 279: 17-21.

- Janssen ML, Duits AA, Turaihi AH, Ackermans L, Leentjens AF, Van KranenMastenbroek V, Oosterloo M, Visser-Vandewalle V, Temel Y. Subthalamic nucleus high frequency stimulation for advanced Parkinson's disease: motor and neuropsychological outcome after 10 years. J Stereotact Funct Neurosurg. 2014 Nov;92:381387.

- Zeef DH, Jahanshahi A, Vlamings R, Casaca-Carreira J, Santegoeds RG, Janssen ML, Oosterloo M, Temel Y. An experimental model for huntington's chorea? Behav Brain Res. 2014 Apr 1;262:31-34.

- Smit JV, Plantinga BR, Janssen ML, Ackermans L, Oosterloo M, Duits A, Leentjens AF, Van Kranen-Mastenbroek V, Van Overbeeke JJ, Temel Y. Diepe hersenstimulatie: de stand van zaken. Tijdschrift voor Neurologie en Neurochirurgie. 2014 Mar; 115(1):20-25.

- Jahanshahi A, Schonfeld L, Janssen ML, Hescham S, Kocabicak E, Steinbusch HW, Van Overbeeke JJ, Temel Y. Electrical stimulation of the motor cortex enhances progenitor cell migration in the adult rat brain. Exp Brain Res. 2013 Nov;231(2):16577.

- Jahanshahi A, Vlamings R, Roon-Mom WV, Faull RL, Waldvogel HJ, Janssen ML, Yakkioui Y, Zeef D, Kocabicak E, Steinbusch HW, Temel Y. Changes in brainstem serotonergic and dopaminergic cell populations in experimental and clinical Huntington's disease. Neuroscience. 2013 May 15;238:71-81.

- Zwartjes DG*, Janssen ML*, Heida T, Van Kranen-Mastenbroek V, Bour LJ, Temel $\mathrm{Y}$,Visser-Vandewalle V, Veltink PH. Cortically evoked potentials in the human subthalamic nucleus. Neurosci Lett. 2013 Feb 28;539:27-31. 
- $\quad$ Temel Y, Hescham SA, Jahanshahi A, Janssen ML, Tan SK, van Overbeeke JJ, Ackermans L, Oosterloo M, Duits A, Leentjens AFG, Lim LW. Neuromodulation in psychiatric disorders. Int Rev Neurobiol. 2012;107:283-314.

- Vlamings R, Zeef DH, Janssen ML, Oosterloo M, Schaper F, Jahanshahi A and Temel Y. Lessons learned from the transgenic Huntington's disease rats. Neural Plast. 2012, Article ID 682712, doi:10.1155/2012/682712

- Vlamings R, Benazzouz A, Chetrit J, Janssen ML, Kozan R, Visser-Vandewalle V, Steinbusch HWM, von Hörsten $S$, and Temel Y. Metabolic and electrophysiological changes in the basal ganglia of a transgenic rat model for Huntington's disease. Neurobiol Dis. 2012 Nov; 48(3):488-494.

- Zwartjes DG, Heida T, Feirabend HK, Janssen ML, Visser-Vandewalle V, Martens HC and Veltink PH. Motor cortex stimulation for Parkinson's disease: a modelling study. J. Neural Eng. 2012 Aug 10;9(5):056005.

- Brunenberg EJ, Moeskops P, Backes WH, Pollo C, Cammoun L, Vilanova A, Janssen ML, Visser-Vandewalle VE, Ter Haar Romeny BM, Thiran JP, Platel B. Structural and resting state functional connectivity of the subthalamic nucleus: Identification of motor STN parts and the hyperdirect pathway. PloS ONE. 2012;7(6):e39061.

- Janssen ML*, Zwartjes DG*, Temel Y, Kranen-Mastenbroek V, Duits A, Bour L, Veltink $P$, Heida T, Visser-Vandewalle $V$. Subthalamic nucleus neuronal responses to single cortical stimuli in a Parkinson's disease patient during stereotactic surgery. Mov Disord 2012 Mar;27(3):435-8.

- Janssen ML, Zwartjes DG, Tan SK, Vlamings R, Jahanshahi A, Heida T, Hoogland G, Steinbusch $\mathrm{H}$, Visser-Vandewalle $\mathrm{V}$, and Temel Y. Mild dopaminergic lesions are accompanied by robust changes in subthalamic nucleus activity. Neurosci Lett. 2012 Febr;508(2):101-105.

- Mencarelli C, Bode GH, Vlamings R, Janssen ML, Losen M, De Baets MH, Steinbusch HWM, Temel Y, Martinez-Martinez P. Unchanged expression of the Ceramide transfer protein in the acute 6-OHDA neurodegenerative model. Neurosci Lett. 2012 Jan 6;506(1):39-43.

- Westin JE, Janssen ML, Sager TN, Temel Y. Automated gait analysis in bilateral Parkinsonian rats and the role of L-DOPA therapy. Behav Brain Res. 2012 Jan;226(2):519-528.

- Zeef DH, Vlamings R, Lim LW, Tan S, Janssen ML, Jahanshahi A, Hoogland G, Prickaerts J, Steinbusch H, Temel Y. Motor and non-motor behaviour in experimental Huntington's disease. Behav Brain Res. 2012 Jan;226(2):435-439.

- Tan SK, Janssen ML, Jahanshahi A, Chouliaras L, Visser-Vandewalle V, Lim LW, Steinbusch HW, Sharp T, Temel Y. High frequency stimulation of the subthalamic nucleus increases c-fos immunoreactivity in the dorsal raphe nucleus and afferent brain regions. J Psychiatr Res. 2011 Oct;45(10):1307-15. 
- Cornips EM, Janssen ML, Beuls EA. Thoracic disc herniation and acute myelopathy: clinical presentation, neuroimaging findings, surgical considerations, and outcome. J Neurosurg Spine. 2011 Apr;14(4):520-8.

- Lim LW, Blokland A, van Duinen M, Visser-Vandewalle V, Tan S, Vlamings R, Janssen M, Jahanshahi A, Aziz-Mohammadi M, Steinbusch HW, Schruers K, Temel Y. Increased plasma corticosterone levels after periaqueductal gray stimulation-induced escape reaction or panic attacks in rats. Behav Brain Res. 2011 Apr;218(2):301-7.

- Tan SK, Vlamings R, Lim L, Sesia T, Janssen ML, Steinbusch HW, Visser-Vandewalle $\checkmark$, Temel Y. Experimental deep brain stimulation in animal models. Neurosurgery. 2010 Oct;67(4):1073-80.

- Jahanshahi A, Vlamings R, Kaya AH, Lim LW, Janssen ML, Tan S, Visser-Vandewalle $\checkmark$, Steinbusch HW, Temel Y. Hyperdopaminergic status in experimental Huntington disease. J Neuropathol Exp Neurol. 2010 Sep;69(9):910-7.

- Janssen ML, Visser-Vandewalle V, Temel Y. Cortico-subthalamic projections in the rat. J Exp Clin Med. 2010 Mar;27(1):4-12.

*shared first author

\section{Oral presentations}

- Efficacy of rostral and caudal PPN stimulation in an experimental rat model for Parkinson's disease based on the functional STN-PPN connectivity. European Society for Stereotactic and Functional Neurosurgery, Maastricht, The Netherlands 2014.

- Finding the right spot for DBS in Parkinson's disease. Biomedica Summit. Liege, Belgium 2012.

- Motor, limbic and associative cortico-subthalamic pathways are not fully segregated in the rat. CGB-CMA meeting, Marseille, France 2011.

- Behavioral side effects in high-frequency stimulation of the subthalamic nucleus. Fellow's day, Montreal Neurological Institute, Canada 2008.

\section{Poster presentations}

- M.L.F. Janssen, D.G.M. Zwartjes, S.K.H. Tan, R. Vlamings, A. Jahanshahi, T. Heida, G. Hoogland, H. Steinbusch, V. Visser-Vandewalle, and Y. Temel. Subthalamic nucleus neuronal responses are modulated by dopamine and evoked responses from motor, limbic and associative cortical areas are not fully segregated in the rat. Movement Disorders. Dublin, Ireland, 2012 and International Basal Ganglia Society, Israel, 2013 
- M.L.F. Janssen, Y. Temel, D.G.M. Zwartjes, C. Delaville, V. Visser-Vandewalle, A. Benazzouz. Mild dopaminergic lesions are accompanied by robust changes in subthalamic nucleus activity. Movement Disorders. Dublin, Ireland, 2012.Selected for guided poster tour

- M.L.F. Janssen, Y. Temel, D.G.M. Zwartjes, V. Visser-Vandewalle, A. Benazzouz. The motor, limbic and associative cortico-subthalamic pathways are not fully segregated in the rat. French Neuroscience Society 2011.

- M.L.F. Janssen, D.G.M. Zwartjes, Y. Temel, V. Van Kranen-Mastenbroek, A. Duits, L. Bour, P.H. Veltink, T. Heida and V. Visser-Vandewalle. Subthalamic responses to motor cortex stimulation: Selective targeting of the subthalamic motor area. Society for Neuroscience 2010. 
